\title{
WestVirginiaUniversity
}

THE RESEARCH REPOSITORY @ WVU

Graduate Theses, Dissertations, and Problem Reports

2009

\section{Circulation control improvements to rotor lift asymmetry due to reverse flow}

\author{
Michael J. Lyons \\ West Virginia University
}

Follow this and additional works at: https://researchrepository.wvu.edu/etd

\section{Recommended Citation}

Lyons, Michael J., "Circulation control improvements to rotor lift asymmetry due to reverse flow" (2009). Graduate Theses, Dissertations, and Problem Reports. 2009.

https://researchrepository.wvu.edu/etd/2009

This Thesis is protected by copyright and/or related rights. It has been brought to you by the The Research Repository @ WVU with permission from the rights-holder(s). You are free to use this Thesis in any way that is permitted by the copyright and related rights legislation that applies to your use. For other uses you must obtain permission from the rights-holder(s) directly, unless additional rights are indicated by a Creative Commons license in the record and/ or on the work itself. This Thesis has been accepted for inclusion in WVU Graduate Theses, Dissertations, and Problem Reports collection by an authorized administrator of The Research Repository @ WVU. For more information, please contact researchrepository@mail.wvu.edu. 


\title{
CIRCULATION CONTROL IMPROVEMENTS TO ROTOR LIFT ASYMMETRY DUE TO REVERSE FlOW
}

\author{
by \\ Michael J. Lyons, BSAE, BSME \\ Thesis Submitted to the \\ College of Engineering and Mineral Resources \\ at West Virginia University \\ in partial fulfillment of the requirements \\ for the degree of \\ Master of Science \\ in \\ Aerospace Engineering \\ Approved by \\ Dr. James E. Smith, Committee Chairperson \\ Dr. Victor H. Mucino \\ Dr. Gerald Angle II \\ Department of Mechanical and Aerospace Engineering \\ Morgantown, WV \\ 2009 \\ Keywords: Circulation Control, Reverse Flow \\ Copyright 2008 Michael J. Lyons
}




\title{
Abstract \\ Circulation Control Improvements to Rotor Lift Asymmetry Due to Reverse Flow
}

\author{
Michael J. Lyons \\ West Virginia University, Morgantown, WV, 26506
}

Circulation control has been applied to airfoils since the late 1960's, and has been proven to change the aerodynamic performance by altering the interaction of the streamlines without changing the physical characteristics of the airfoil itself. This has many applications in fluid dynamics; the focus of this application is for the replacement of the conventional helicopter rotor blade system with a fly-by-wire, active circulation controlled system. Conventional helicopters use a swashplate and a series of mechanical linkages, bearings, and dampers to create a fully articulated rotor hub system. This system is required to achieve the blade characteristics required for stable flight. The need for such a system stems from the asymmetric lift developed in maneuvering flight conditions, which requires the angle of attack of the blades to be changed based on the rotational position within the rotor plane, also known as the azimuth angle. By alternatively activating blowing slots along the leading and trailing edges of the airfoil, the aerodynamic parameters (i.e. lift and drag) can be changed, effectively changing the angle of attack through streamline alteration thus eliminating the need for physical blade pitch changes.

Mathematical models/codes are used to model and simulate the complex blade dynamics of a full-scale rotorcraft. Many of these codes use a blade element method that separates the rotor into small segments and evaluates the aerodynamic characteristics of these elements as two-dimensional airfoils at different local conditions. These local conditions include, but are not limited to, extreme angles of attack, reverse flow, compressibility effects, dynamic effects, and other aerodynamic phenomena.

This research investigated the reverse flow aerodynamic characteristics of a 10:1 elliptical airfoil with various leading and trailing edge blowing pressures. The testing conditions for the aerodynamic investigation were: angle of attack [154 to 196 degrees]; blowing coefficient [0 to 0.009] and [0 to 0.014] for the leading and trailing edge blowing slots, respectively; and Mach number [0.073 and 0.109].

It is concluded that the potential exists for increasing forward flight speeds for helicopters using circulation control in the reverse flow regions of the helicopter rotor environment. Specifically, it is concluded that positively altering the aerodynamic characteristics, primarily the lift coefficient, in reverse flow, is possible through circulation control. Through this investigation, the general trends were found for the aerodynamic characteristics of a 10:1 circulation controlled elliptical airfoil in reverse flow. These trends led to the selection of blowing configurations to decrease the asymmetric loading condition based on the condition of the local blade environment. 
[This page is left intentionally blank] 


\section{Acknowledgements}

The author would like to thank all who have provided their assistance in any part of the research documented here. The patience and contribution of time by all those involved has been greatly appreciated. Specifically, I would like to thank Steven Hard, Christina Yarborough, Andrew Reeder, Jordan Berry, and Joe Allen for their assistance in data collection. I would also like to thank Chad Panther for keeping my sanity with the verification of concepts and mathematics. Thanks are due to Dr. Gerald Angle and Jonathan Kweder for their expertise with the low-speed wind tunnel and Jay Wilhelm and Justin Smith for data acquisition support. I would like to thank Dr. James Smith for direction and Dr. John Kuhlman for his academic input as well as the faculty and staff of West Virginia University for the many areas of support over the last few years and the opportunity to succeed. Lastly, I would like to thank my parents James and Judy Lyons as well as other family members for their persistence and unwavering belief in my efforts. 


\section{Table of Contents}

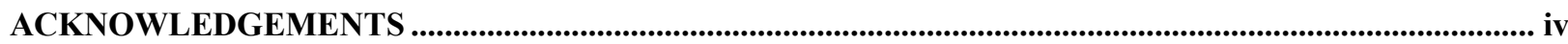

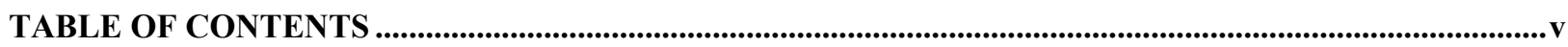

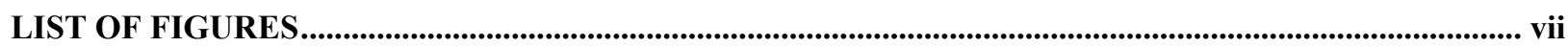

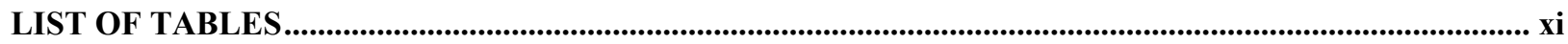

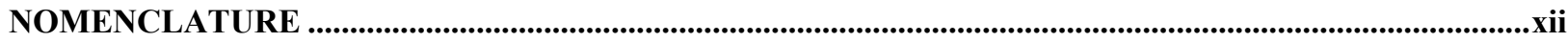

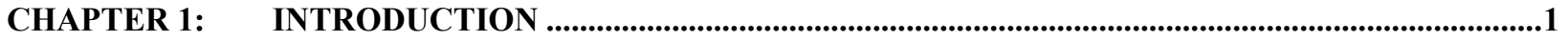

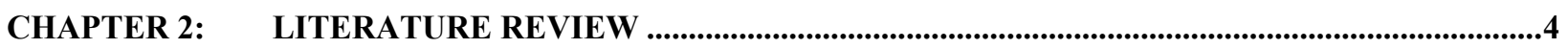

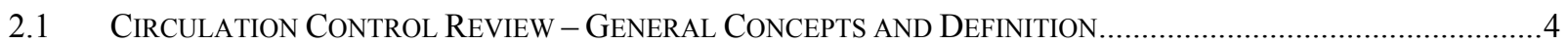

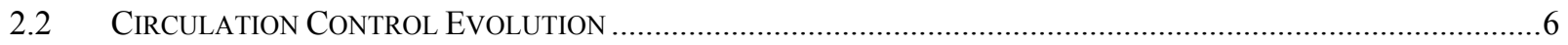

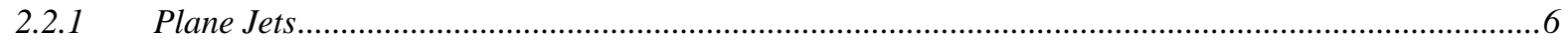

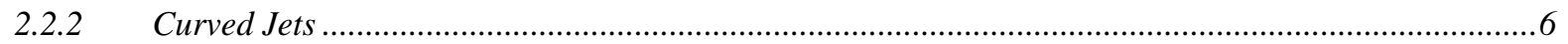

2.2.3 Circulation Controlled Airfoils (Curved Jets in External Flows) ................................................... 8

CHAPTER 3: $\quad$ EXPERIMENTAL SETUP REVIEW ….........................................................................11

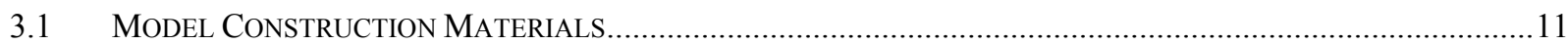

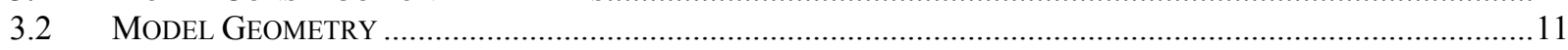

3.3 MEASUREMENT AND CALCULATION TECHNIQUES FOR IMPORTANT NON-DIMENSIONAL PARAMETERS .......12

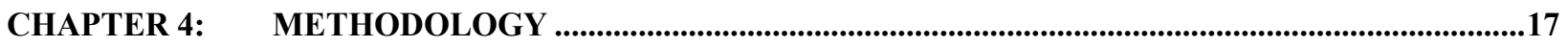

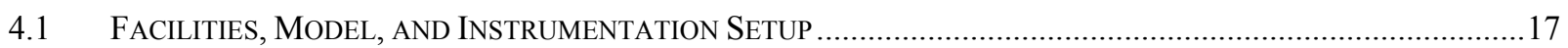

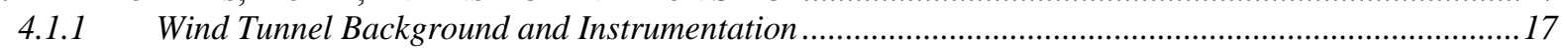

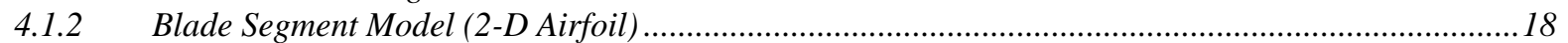

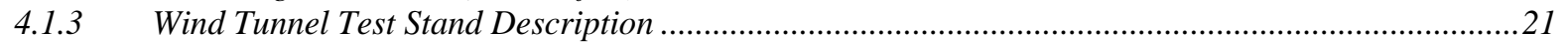

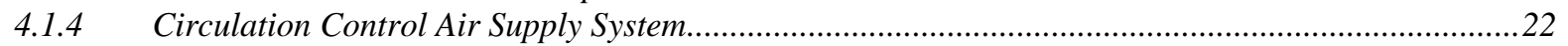

4.2 FACILITIES, MODEL, AND INSTRUMENT EXPERIMENTAL PROCEDURES …..................................................23

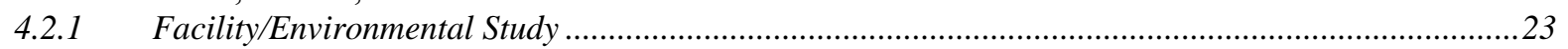

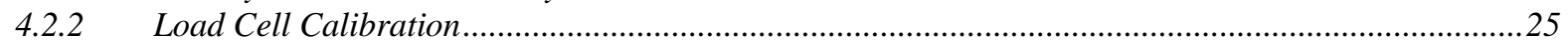

4.2.3 Angle-of-Attack Tare / Final Force Calculation...................................................................................26

4.2.4 Lift and Drag Coefficient Calculation and Correction....................................................................27

4.2.5 Jet Velocity and Blowing Coefficient Determination ....................................................................... 31

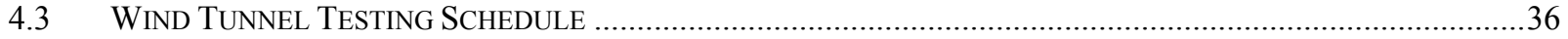

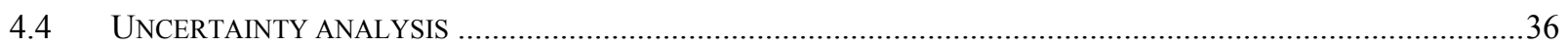

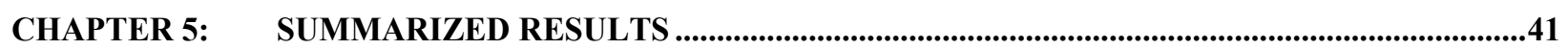

5.1 RESUlTS FOR LIFT AND DRAg COEFFICIENTS UNDER THE NO-BLOWING CONDITION ..................................41

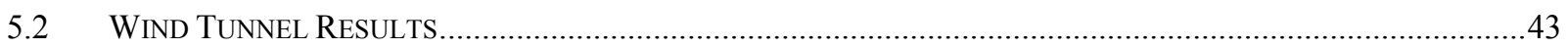

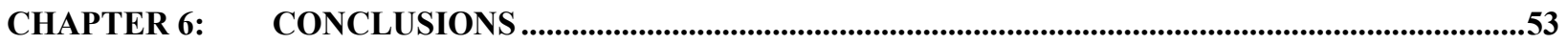

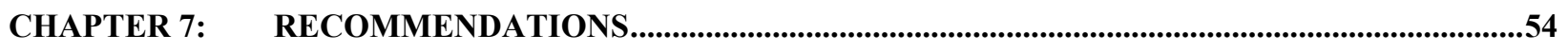

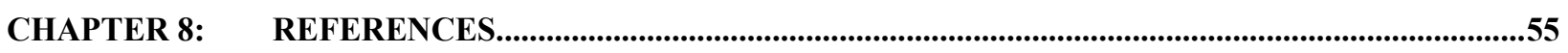

APPENDIX A - LOAD CELL CALIBRATION AND FORCE CALCULATION EXPERIMENTATION . A-1

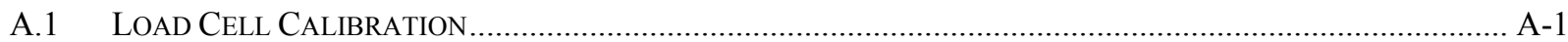

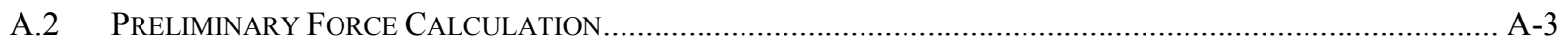

A.3 7-Point LOAD CELl CALIBRATION CURVES AND ERRor CALCULATION.................................................. A-7

A.4 LoAd CELl Multiple-LoAding VAlidation ............................................................................ A-13

APPENDIX B - DETAILED RESULTS FOR LIFT AND DRAG COEFFICIENT ...........................................B-1 


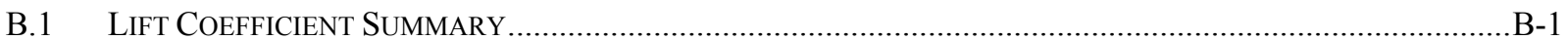

B.2 Lift CoEfFicient - Trailing Edge VARYING - Average TARE METHOD .................................................

B.3 LIFT COEFFICIENT - TRAILING EDGE VARYING - INDIVIDUAL TARES METHOD ......................................... B-7

B.4 LIFT COEFFICIENT CHANGE - TRAILING EDGE VARYING ......................................................................

B.5 LifT COEFFICIENT - LEADING EdGe VARYING - AvERAGE TARE METHOD ................................................ B-14

B.6 LIFT COEFFICIENT CHANGE - LEADING EdGE VARYING ......................................................................... B-17

B.7 LifT COEFFICIENT - LEADING EDGE VARYING - INDIVIDUAL TARES METHOD.......................................... B-19

B.8 Drag COEFFICIENT - TRAILING Edge VARYING - AVERAgE TARE METHOD ............................................

B.9 DRAG COEFFICIENT - TRAILING EDGE VARYING - INDIVIDUAL TARES METHOD ....................................B-25

B.10 DRAG COEFFICIENT CHANGE - TRAILING EDGE VARYING.................................................................

B.11 Drag COEFFICIENT - LEAdING EDGE VARYING - AVERAge TARE METHOD ...................................... B-31

B.12 DRAG COEFFICIENT - LEADING EDGE VARYING - INDIVIDUAL TARES METHOD …...............................B-34

B.13 DRAG COEFFICIENT CHANGE - LEADING EDGE VARYING …...............................................................3

APPENDIX C - SHORT SUMMARY FOR MINIMUM/MAXIMUM LIFT COEFFICIENTS AND STALL

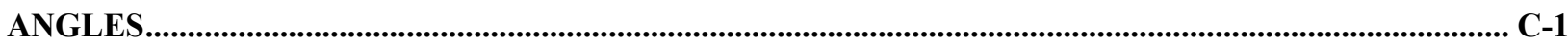

APPENDIX D - MATLAB PROGRAMS FOR USE WITH WVU CLOSED LOOP WIND TUNNEL DATA

ACQUISITION SOFTWARE .............................................................................................................................. D-1 


\section{List of Figures}

Figure 1.1: Growth of Reversed Flow Regions with Increasing Advance Ratios [Wood, 1981], Note: Reversed Flow Occurs Over the Portion of the Blade within the Operating Advance Ratio Circle ....................................................2 Figure 2.1: Proposed Coanda Effect Explanation [Wood, 1981] ...................................................................4 Figure 2.2: Performance Trends for a Circulation Controlled Aerofoil [Wood, 1981] ...........................................5 Figure 2.3: Lifting Performance of the Airfoil as a Function of Effective Incidence for Trailing Edge Blowing Only [Wood, 1981]....

Figure 2.4: Change in lift coefficient and moment coefficient vs. blowing coefficient at $\mathrm{M}=0.3, \alpha=+6 \mathrm{deg}$ [Alexander and Anders, 2005].

Figure 2.5: $\mathrm{C}_{1}$ vs. $\mathrm{C}_{\mu}$, angle of attack effect, upper slot blowing, Coanda (2.98:1), slot (h/c =0.0012) [Alexander and Anders, 2005].

Figure 3.1: Mass Flow Measurement, Critical Venturi System [Alexander and Anders, 2005] ...........................14

Figure 3.2: Estimation of Jet Velocity by Three Methods [Wood, 1981] .................................................15

Figure 4.1: WVU Closed Loop Wind Tunnel (Not to scale) .............................................................................18

Figure 4.2: CAD Drawing for Proof of Concept Model of a Helicopter Blade with Circulation Control [Angle, 2006]

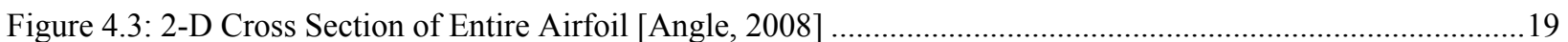

Figure 4.4: Internal Structure of the Blade Segment [Angle, 2008] ...............................................................20

Figure 4.5: Internal Ports and Porous Walls of the Blade Element [Angle, 2008] ...........................................20

Figure 4.6: Test Section and Stand Design in as Developed in Pro-Engineer [Lyons, 2008] ...............................21

Figure 4.7: Rotating Assembly and Load Cell Setup ................................................................................22

Figure 4.8: Supply Air System and Measurement Locations (Not drawn to scale or shape)..................................22

Figure 4.9: X-Axis Logarithmic Plot of the Average Frequency Components [Lyons, 2008] .............................24

Figure 4.10: Exaggerated Deflection of the WVU Circulation Controlled model stand [Lyons, 2008] ...................25

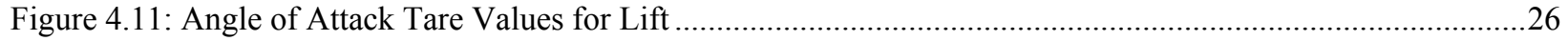

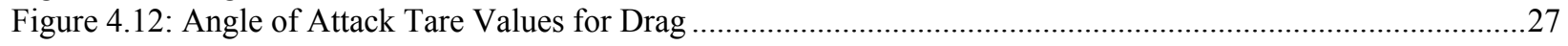

Figure 4.13: Base Factors from Various Airfoils (Generated from Allen and Vincenti, 1949) ................................30

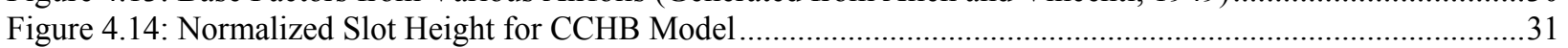

Figure 4.15: Trailing Edge Normalized Velocity Profile ................................................................................32

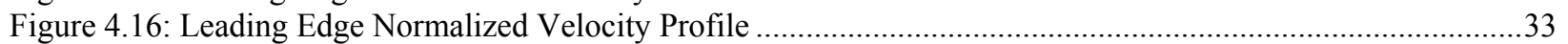

Figure 4.17: Blowing Slot Jet Maximum Velocity Direction .....................................................................33

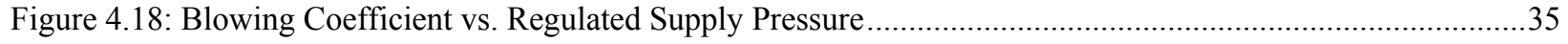

Figure 4.19: Lift Coefficient Error Bar Plot (Tunnel Velocity $=80 \mathrm{fps}$, no blowing) ........................................40

Figure 4.20: Drag Coefficient Error Bar Plot (Tunnel Velocity $=80 \mathrm{fps}$, no blowing) ........................................40

Figure 5.1: Lift Coefficient vs. Angle of Attack, No Blowing Comparison.........................................................42

Figure 5.2: Drag Coefficient vs. Angle of Attack, No Blowing Comparison..........................................................42

Figure 5.3: Change in Lift Coefficient vs. Leading Edge Blowing Coefficient vs. Negative Angle of Attack ...........44

Figure 5.4: Change in Lift Coefficient vs. Leading Edge Blowing Coefficient vs. Positive Angle of Attack............44

Figure 5.5: Change in Lift Coefficient vs. Leading Edge Blowing Coefficient vs. Negative Angle of Attack, Trailing

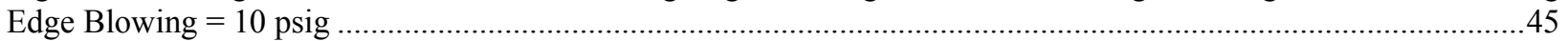

Figure 5.6: Change in Lift Coefficient vs. Leading Edge Blowing Coefficient vs. Positive Angle of Attack, Trailing

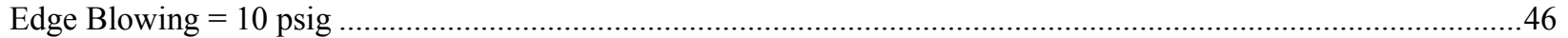

Figure 5.7: Change in Lift Coefficient vs. Leading Edge Blowing Coefficient vs. Negative Angle of Attack, Trailing

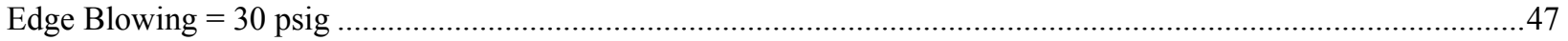

Figure 5.8: Change in Lift Coefficient vs. Leading Edge Blowing Coefficient vs. Positive Angle of Attack, Trailing

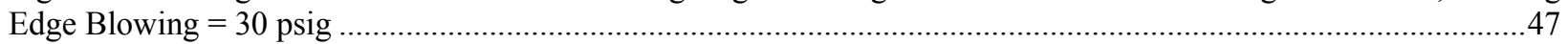

Figure 5.9: Change in Lift Coefficient vs. Trailing Edge Blowing Coefficient vs. Negative Angle of Attack ...........48

Figure 5.10: Change in Lift Coefficient vs. Trailing Edge Blowing Coefficient vs. Positive Angle of Attack..........49

Figure 5.11: Change in Lift Coefficient vs. Trailing Edge Blowing Coefficient vs. Negative Angle of Attack,

Leading Edge Blowing = 10 psig .............................................................................................49

Figure 5.12: Change in Lift Coefficient vs. Trailing Edge Blowing Coefficient vs. Positive Angle of Attack, Leading

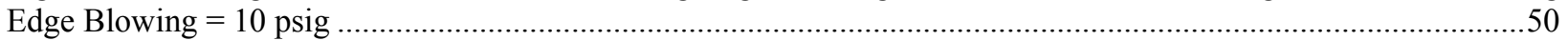

Figure 5.13: Change in Lift Coefficient vs. Trailing Edge Blowing Coefficient vs. Negative Angle of Attack,

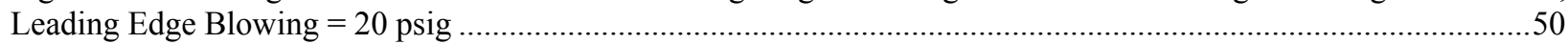


Figure 5.14: Change in Lift Coefficient vs. Trailing Edge Blowing Coefficient vs. Positive Angle of Attack, Leading Edge Blowing $=20 \mathrm{psig}$

Figure A.1: Calibration Setup for Top Load Cells ............................................................................................. A-2

Figure A.2: Top Lift loaded Calibration Curves...................................................................................... A-3

Figure A.3: Top Drag loaded Calibration Curves.................................................................................... A-7

Figure A.4: Bottom Lift Loaded Calibration Curves ............................................................................................ A-8

Figure A.5: Bottom Drag Loaded Voltage Readings.......................................................................................... A-9

Figure B.6: Lift Coefficient vs. Leading Edge Blowing Coefficient vs. Angle of Attack ....................................... B-1

Figure B.7: Lift Coefficient vs. Leading Edge Blowing Coefficient vs. Angle of Attack, Trailing Edge Blowing $=10$

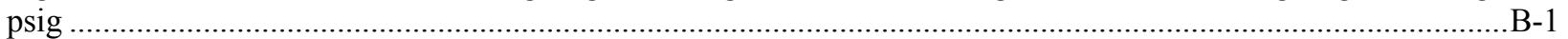

Figure B.8: Lift Coefficient vs. Leading Edge Blowing Coefficient vs. Angle of Attack, Trailing Edge Blowing $=30$

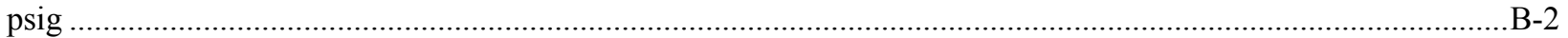

Figure B.9: Lift Coefficient vs. Trailing Edge Blowing Coefficient vs. Angle of Attack ......................................B-2

Figure B.10: Lift Coefficient vs. Trailing Edge Blowing Coefficient vs. Angle of Attack, Leading Edge Blowing =

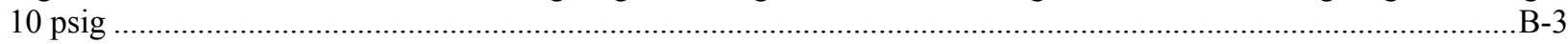

Figure B.11: Lift Coefficient vs. Trailing Edge Blowing Coefficient vs. Angle of Attack, Leading Edge Blowing =

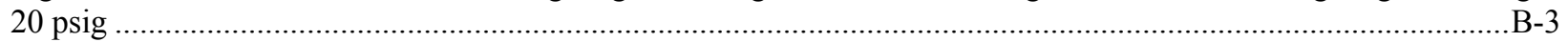

Figure B.12: Tunnel Velocity $=80 \mathrm{fps}$, Trailing Edge Blowing Only, Lift Coefficient vs. Angle of Attack (average

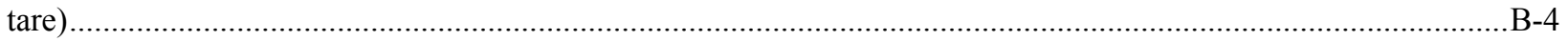
Figure B.13: Tunnel Velocity $=120 \mathrm{fps}$, Trailing Edge Blowing Only, Lift Coefficient vs. Angle of Attack (average

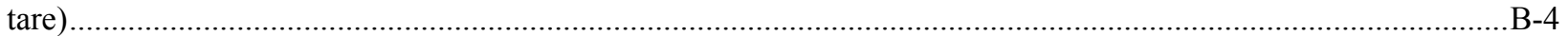
Figure B.14: Tunnel Velocity $=80 \mathrm{fps}$, Trailing Edge Varying and Leading Edge Blowing at $10 \mathrm{psi}$, Lift Coefficient vs. Angle of Attack (average tare) …….................................................................................................. Figure B.15: Tunnel Velocity $=120 \mathrm{fps}$, Trailing Edge Varying and Leading Edge Blowing at $10 \mathrm{psi}$, Lift Coefficient vs. Angle of Attack (average tare) .................................................................................................... Figure B.16: Tunnel Velocity $=80 \mathrm{fps}$, Trailing Edge Varying and Leading Edge Blowing at $20 \mathrm{psi}$, Lift Coefficient vs. Angle of Attack (average tare) ……................................................................................................... Figure B.17: Tunnel Velocity = $120 \mathrm{fps}$, Trailing Edge Varying and Leading Edge Blowing at $20 \mathrm{psi}$, Lift Coefficient vs. Angle of Attack (average tare) ............................................................................................... Figure B.18: Tunnel Velocity $=80 \mathrm{fps}$, Trailing Edge Blowing Only, Lift Coefficient vs. Angle of Attack (Error

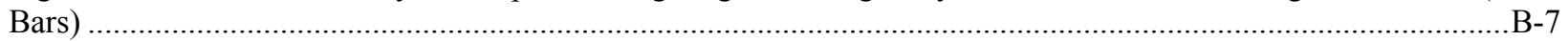
Figure B.19: Tunnel Velocity $=80 \mathrm{fps}$, Trailing Edge Blowing Only, Lift Coefficient vs. Angle of Attack

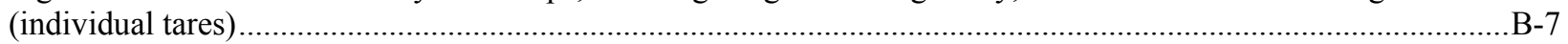
Figure B.20: Tunnel Velocity $=120 \mathrm{fps}$, Trailing Edge Blowing Only, Lift Coefficient vs. Angle of Attack

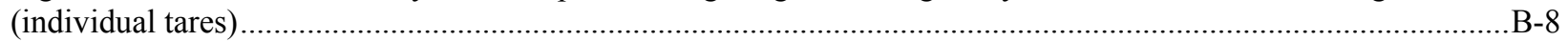
Figure B.21: Tunnel Velocity $=80 \mathrm{fps}$, Trailing Edge Varying and Leading Edge Blowing at $10 \mathrm{psi}$, Lift Coefficient vs. Angle of Attack (individual tares) ............................................................................................................ Figure B.22: Tunnel Velocity $=120 \mathrm{fps}$, Trailing Edge Varying and Leading Edge Blowing at 10 psi, Lift Coefficient vs. Angle of Attack (individual tares) ................................................................................................. B-9 Figure B.23: Tunnel Velocity $=80 \mathrm{fps}$, Trailing Edge Varying and Leading Edge Blowing at $20 \mathrm{psi}$, Lift Coefficient

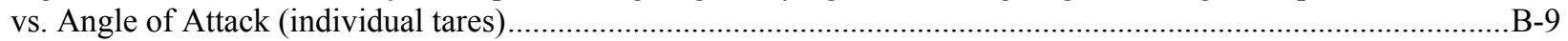
Figure B.24: Tunnel Velocity $=120 \mathrm{fps}$, Trailing Edge Varying and Leading Edge Blowing at $20 \mathrm{psi}$, Lift Coefficient vs. Angle of Attack (individual tares) ................................................................................................. Figure B.25: Tunnel Velocity $=80 \mathrm{fps}$, Change in Lift Coefficient vs. Angle of Attack, Trailing Edge Blowing Only Figure B 26: Tunnel Velocity $=120 \mathrm{fps}$, Change in Lift Coefficient vs. Angle of Attack, Trailing Edge Blowing

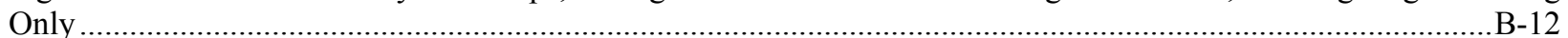
Figure B.27: Tunnel Velocity $=80 \mathrm{fps}$, Change in Lift Coefficient vs. Angle of Attack, Trailing Edge Varying,

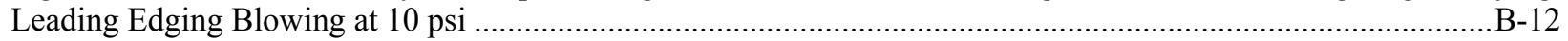
Figure B.28: Tunnel Velocity $=120 \mathrm{fps}$, Change in Lift Coefficient vs. Angle of Attack, Trailing Edge Varying, Leading Edging Blowing at $10 \mathrm{psi}$......................................................................................................... Figure B.29: Tunnel Velocity $=80 \mathrm{fps}$, Change in Lift Coefficient vs. Angle of Attack, Trailing Edge Varying,

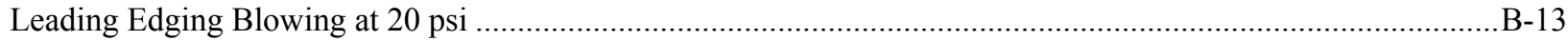
Figure B.30: Tunnel Velocity = $120 \mathrm{fps}$, Change in Lift Coefficient vs. Angle of Attack, Trailing Edge Varying, Leading Edging Blowing at 20 psi B-13 
Figure B.31: Tunnel Velocity $=80$ fps, Change in Lift Coefficient vs. Angle of Attack, Leading Edge Blowing Only (average tare) B-14 Figure B.32: Tunnel Velocity $=120 \mathrm{fps}$, Change in Lift Coefficient vs. Angle of Attack, Leading Edge Blowing

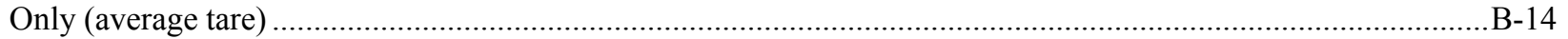
Figure B.33: Tunnel Velocity $=80 \mathrm{fps}$, Change in Lift Coefficient vs. Angle of Attack, Leading Edge Varying, Trailing Edge Blowing at 10 psi (average tare) ................................................................................................ Figure B.34: Tunnel Velocity = $120 \mathrm{fps}$, Change in Lift Coefficient vs. Angle of Attack, Leading Edge Varying, Trailing Edge Blowing at 10 psi (average tare) .............................................................................................. 15 Figure B.35: Tunnel Velocity $=80 \mathrm{fps}$, Change in Lift Coefficient vs. Angle of Attack, Leading Edge Varying, Trailing Edge Blowing at 30 psi (average tare) ........................................................................................ 16 Figure B.36: Tunnel Velocity $=120 \mathrm{fps}$, Change in Lift Coefficient vs. Angle of Attack, Leading Edge Varying,

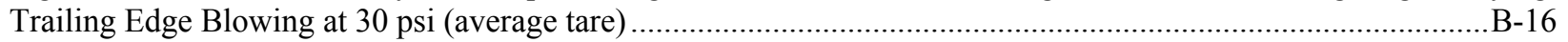
Figure B.37: Tunnel Velocity $=80$ fps, Change in lift coefficient, Leading Edge Blowing Only .........................B-17 Figure B.38: Tunnel Velocity $=120 \mathrm{fps}$, Change in lift coefficient, Leading Edge Blowing Only .......................B-17 Figure B.39: Tunnel Velocity $=80 \mathrm{fps}$, Change in lift coefficient, Leading Edge Varying, Trailing Edge Blowing at

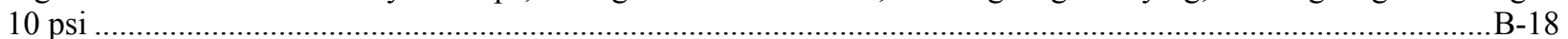
Figure B.40: Tunnel Velocity $=120 \mathrm{fps}$, Change in lift coefficient, Leading Edge Varying, Trailing Edge Blowing at

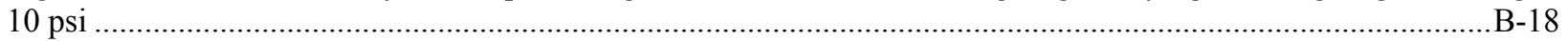
Figure B.41: Tunnel Velocity $=80 \mathrm{fps}$, Change in lift coefficient, Leading Edge Varying, Trailing Edge Blowing at

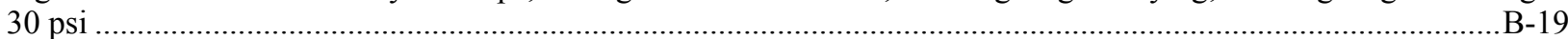
Figure B.42: Tunnel Velocity = 120 fps, Change in lift coefficient, Leading Edge Varying, Trailing Edge Blowing at

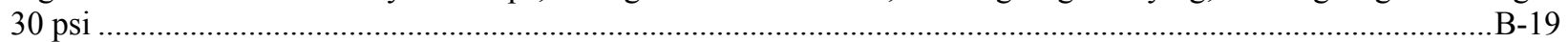
Figure B.43: Tunnel Velocity $=80 \mathrm{fps}$, Lift Coefficient vs. Angle of Attack, Leading Edge Blowing Only

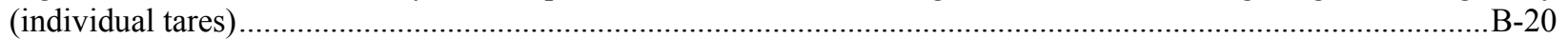
Figure B.44: Tunnel Velocity $=120 \mathrm{fps}$, Lift Coefficient vs. Angle of Attack, Leading Edge Blowing Only

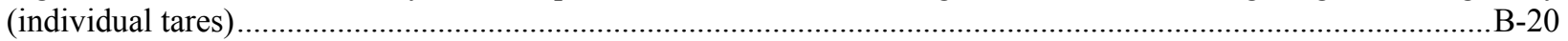
Figure B.45: Tunnel Velocity $=80 \mathrm{fps}$, Lift Coefficient vs. Angle of Attack, Leading Edge Varying, Trailing

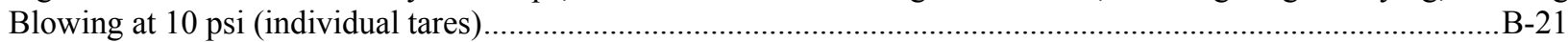
Figure B.46: Tunnel Velocity = 120 fps, Lift Coefficient vs. Angle of Attack, Leading Edge Varying, Trailing Blowing at $10 \mathrm{psi}$ (individual tares)..................................................................................................... Figure B.47: Tunnel Velocity $=80 \mathrm{fps}$, Lift Coefficient vs. Angle of Attack, Leading Edge Varying, Trailing

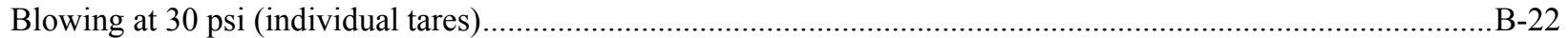
Figure B.48: Tunnel Velocity $=120$ fps, Lift Coefficient vs. Angle of Attack, Leading Edge Varying, Trailing

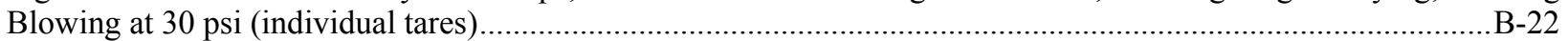
Figure B.49: Tunnel Velocity $=80 \mathrm{fps}$, Drag Coefficient vs. Angle of Attack, Trailing Edge Blowing Only (average tare) Figure B.50: Tunnel Velocity $=120 \mathrm{fps}$, Drag Coefficient vs. Angle of Attack, Trailing Edge Blowing Only

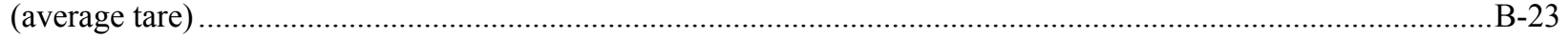
Figure B.51: Tunnel Velocity $=80 \mathrm{fps}$, Drag Coefficient vs. Angle of Attack, Trailing Edge Varying, Leading Edge

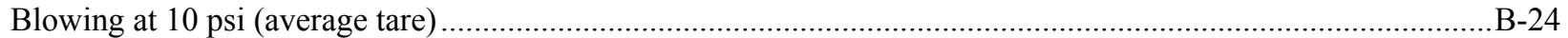
Figure B.52: Tunnel Velocity $=120 \mathrm{fps}$, Drag Coefficient vs. Angle of Attack, Trailing Edge Varying, Leading

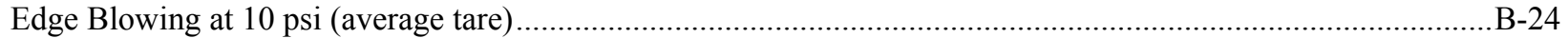
Figure B.53: Tunnel Velocity = 80 fps, Drag Coefficient vs. Angle of Attack, Trailing Edge Varying, Leading Edge

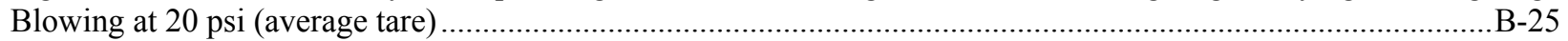
Figure B.54: Tunnel Velocity $=120 \mathrm{fps}$, Drag Coefficient vs. Angle of Attack, Trailing Edge Varying, Leading

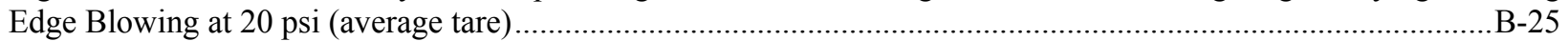
Figure B.55: Tunnel Velocity $=80 \mathrm{fps}$, Drag Coefficient vs. Angle of Attack, Trailing Edge Blowing Only

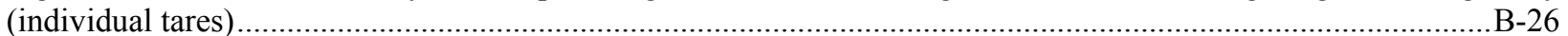
Figure B.56: Tunnel Velocity $=120$ fps, Drag Coefficient vs. Angle of Attack, Trailing Edge Blowing Only (individual tares) .............................................................................................................................. Figure B.57: Tunnel Velocity $=80 \mathrm{fps}$, Drag Coefficient vs. Angle of Attack, Trailing Edge Varying, Leading Edge

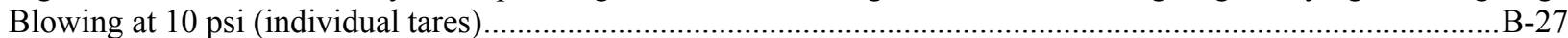
Figure B.58: Tunnel Velocity $=120 \mathrm{fps}$, Drag Coefficient vs. Angle of Attack, Trailing Edge Varying, Leading

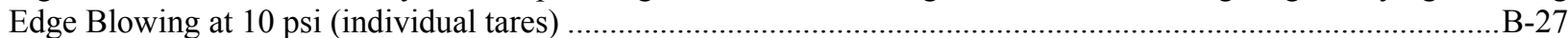
Figure B.59: Tunnel Velocity $=80 \mathrm{fps}$, Drag Coefficient vs. Angle of Attack, Trailing Edge Varying, Leading Edge

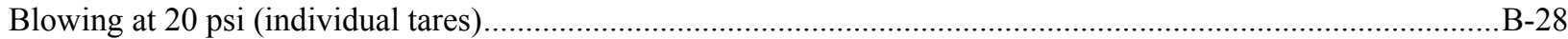


Figure B.60: Tunnel Velocity $=120$ fps, Drag Coefficient vs. Angle of Attack, Trailing Edge Varying, Leading Edge Blowing at $20 \mathrm{psi}$ (individual tares) ................................................................................................ 28 Figure B.61: Tunnel Velocity $=80 \mathrm{fps}$, Drag Coefficient Change vs. Angle of Attack, Trailing Edge Blowing Only B-29

Figure B.62: Tunnel Velocity $=120$ fps, Drag Coefficient Change vs. Angle of Attack, Trailing Edge Blowing Only

Figure B.63: Tunnel Velocity = 80 fps, Drag Coefficient Change Leading Edge Blowing at $10 \mathrm{psi}$ Figure B.64: Tunnel Velocity $=120$ fps, Drag Coefficient Change vs. Angle of Attack, Trailing Edge Varying, Leading Edge Blowing at 10 psi......................................................................................................... Figure B.65: Tunnel Velocity $=80 \mathrm{fps}$, Drag Coefficient Change vs. Angle of Attack, Trailing Edge Varying, Leading Edge Blowing at 20 psi............................................................................................................. Figure B.66: Tunnel Velocity $=120 \mathrm{fps}$, Drag Coefficient Change vs. Angle of Attack, Trailing Edge Varying, Leading Edge Blowing at 20 psi........................................................................................................... B-31 Figure B.67: Tunnel Velocity $=80 \mathrm{fps}$, Drag Coefficient vs. Angle of Attack, Leading Edge Blowing Only (average

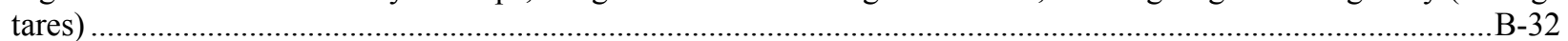
Figure B.68: Tunnel Velocity $=120$ fps, Drag Coefficient vs. Angle of Attack, Leading Edge Blowing Only (average tares) ........................................................................................................................... Figure B.69: Tunnel Velocity $=80 \mathrm{fps}$, Drag Coefficient vs. Angle of Attack, Leading Edge Varying, Trailing Edge

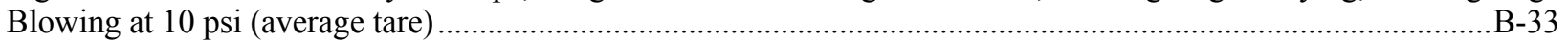
Figure B.70: Tunnel Velocity $=120$ fps, Drag Coefficient vs. Angle of Attack, Leading Edge Varying, Trailing

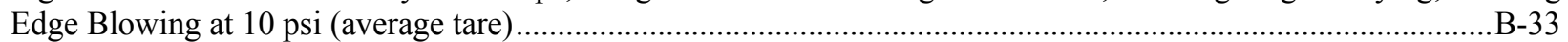
Figure B.71: Tunnel Velocity = $80 \mathrm{fps}$, Drag Coefficient vs. Angle of Attack, Leading Edge Varying, Trailing Edge

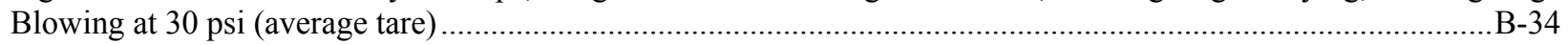
Figure B.72: Tunnel Velocity $=120$ fps, Drag Coefficient vs. Angle of Attack, Leading Edge Varying, Trailing Edge Blowing at $30 \mathrm{psi}$ (average tare) ..................................................................................................... Figure B.73: Tunnel Velocity $=80 \mathrm{fps}$, Drag Coefficient vs. Angle of Attack, Leading Edge Blowing Only

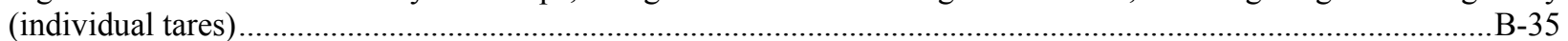
Figure B.74: Tunnel Velocity $=120$ fps, Drag Coefficient vs. Angle of Attack, Leading Edge Blowing Only

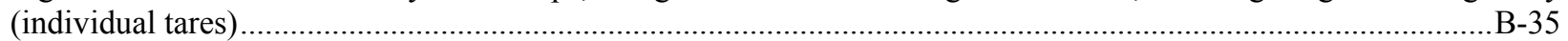
Figure B.75: Tunnel Velocity $=80 \mathrm{fps}$, Drag Coefficient vs. Angle of Attack, Leading Edge Varying, Trailing Edge

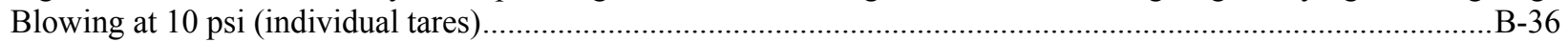
Figure B.76: Tunnel Velocity $=120$ fps, Drag Coefficient vs. Angle of Attack, Leading Edge Varying, Trailing

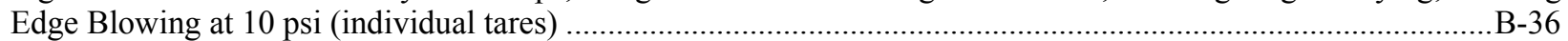
Figure B.77: Tunnel Velocity $=80 \mathrm{fps}$, Drag Coefficient vs. Angle of Attack, Leading Edge Varying, Trailing Edge

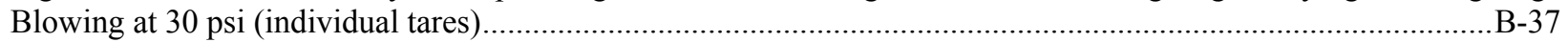
Figure B.78: Tunnel Velocity $=120$ fps, Drag Coefficient vs. Angle of Attack, Leading Edge Varying, Trailing Edge Blowing at $30 \mathrm{psi}$ (individual tares) ................................................................................................ Figure B.79: Tunnel Velocity $=80$ fps, Drag Coefficient Change vs. Angle of Attack, Leading Edge Blowing Only

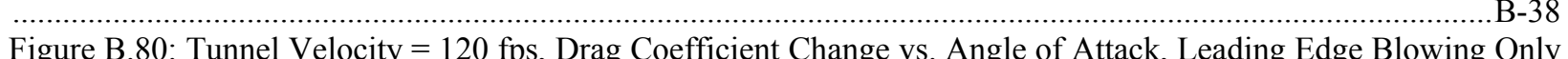

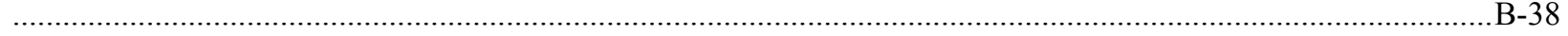

Figure B.81: Tunnel Velocity $=80 \mathrm{fps}$, Drag Coefficient Change vs. Angle of Attack, Leading Edge Varying,

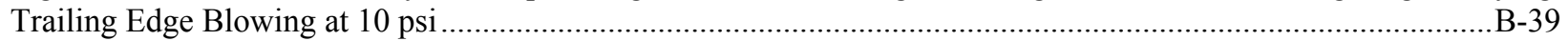
Figure B.82: Tunnel Velocity $=120$ fps, Drag Coefficient Change vs. Angle of Attack, Leading Edge Varying,

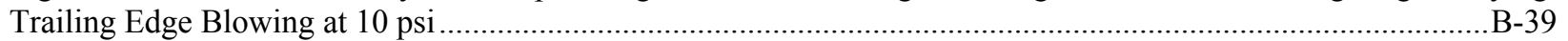
Figure B.83: Tunnel Velocity $=80 \mathrm{fps}$, Drag Coefficient Change vs. Angle of Attack, Leading Edge Varying,

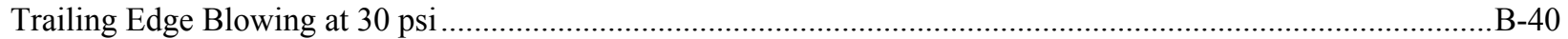
Figure B.84: Tunnel Velocity $=120$ fps, Drag Coefficient Change vs. Angle of Attack, Leading Edge Varying,

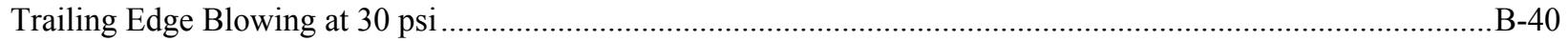




\section{List of Tables}

Table 3.1: Experimental Setup Review and Comparison ................................................................................... 16

Table 4.1: Average Frequency and Voltage Magnitudes for Various Wind Tunnel Components [Lyons, 2008].......23

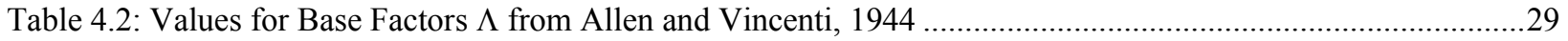

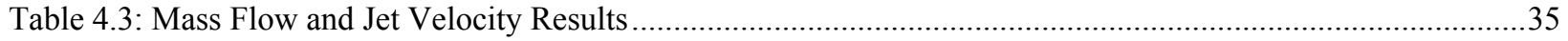

Table 4.4: Area and Velocity Ratios Used in Mass Flow Calculations ...............................................................35

Table 4.5: Lift and Drag Coefficient Uncertainties (Sample: Tunnel Velocity $=80$ fps, No Blowing) ......................38

Table 4.6: Lift and Drag Force Uncertainties (Tunnel Velocity $=80 \mathrm{fps}$, No Blowing) ...........................................39

Table 4.7: Uncertainties of Various Parameters (Tunnel Velocity $=80 \mathrm{fps}$, No Blowing) ......................................39

Table 5.1: Summarized Effects of Various Blowing Configurations in Reverse Flow, Leading or Trailing Edge

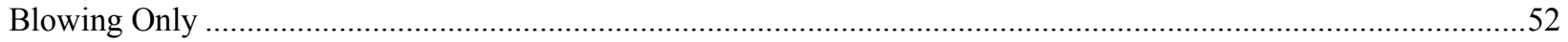

Table 5.2: : Summarized Effects of Various Blowing Configurations in Reverse Flow, Combined Blowing ............52

Table A.1: Description of Calibration Testing Schedule Terms ........................................................................ A-1

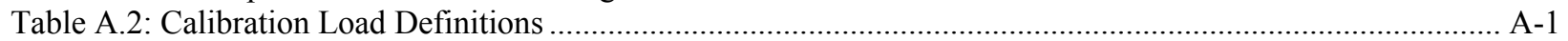

Table A.3: Calibration Testing Schedule for Single Loads ........................................................................... A-2

Table A.4: Example Gain Parameter Calculation (Top Lift Force Applied) ………............................................. A-5

Table A.5: Forces Calculated Using On-Axis Calibration Curves Only ………................................................... A-10

Table A.6: Forces Calculated Using On and Off Axis Calibration Curves Only ............................................... A-11

Table A.7: Forces Calculated Using All Calibration Curves (Matrix) ............................................................. A-12

Table A.8: Error Comparison for Calibration Methods Using Calibration Data ................................................. A-13

Table A.9: Multiple-Loading Validation Using On-Axis Calibration Curves Only ............................................. A-13

Table A.10: Multiple-Loading Validation Using On and Off Axis Calibration Curves ....................................... A-13

Table A.11: Multiple-Loading Validation Using All Axis Calibration .............................................................. A-14

Table A.12: Error Comparison for Calibration Methods Using Multiple-Loading Validation Data ..................... A-14

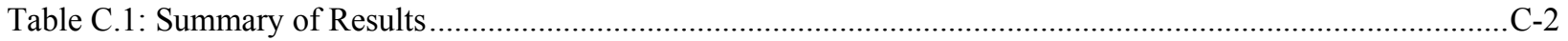

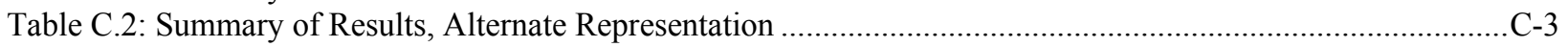




\section{Nomenclature}

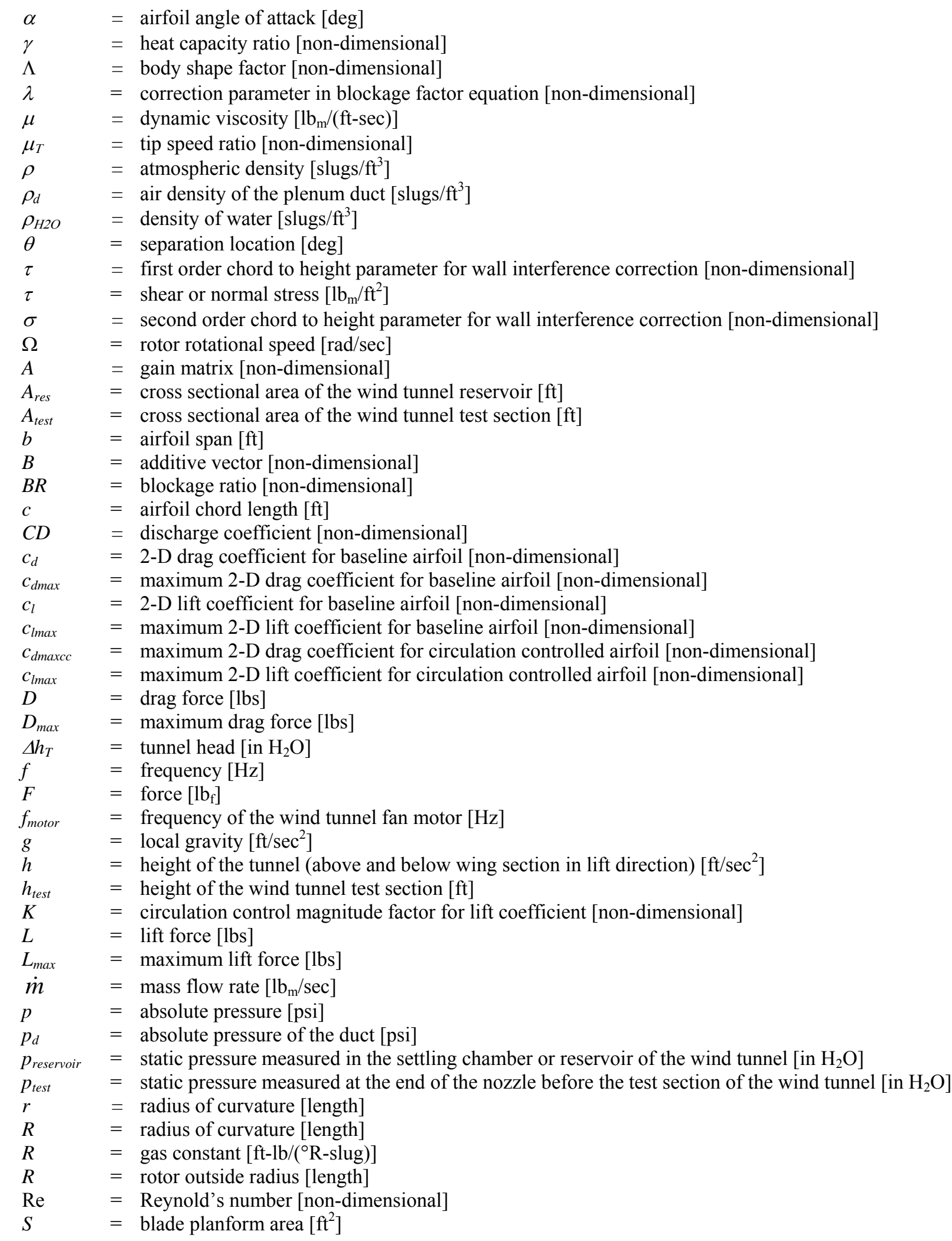




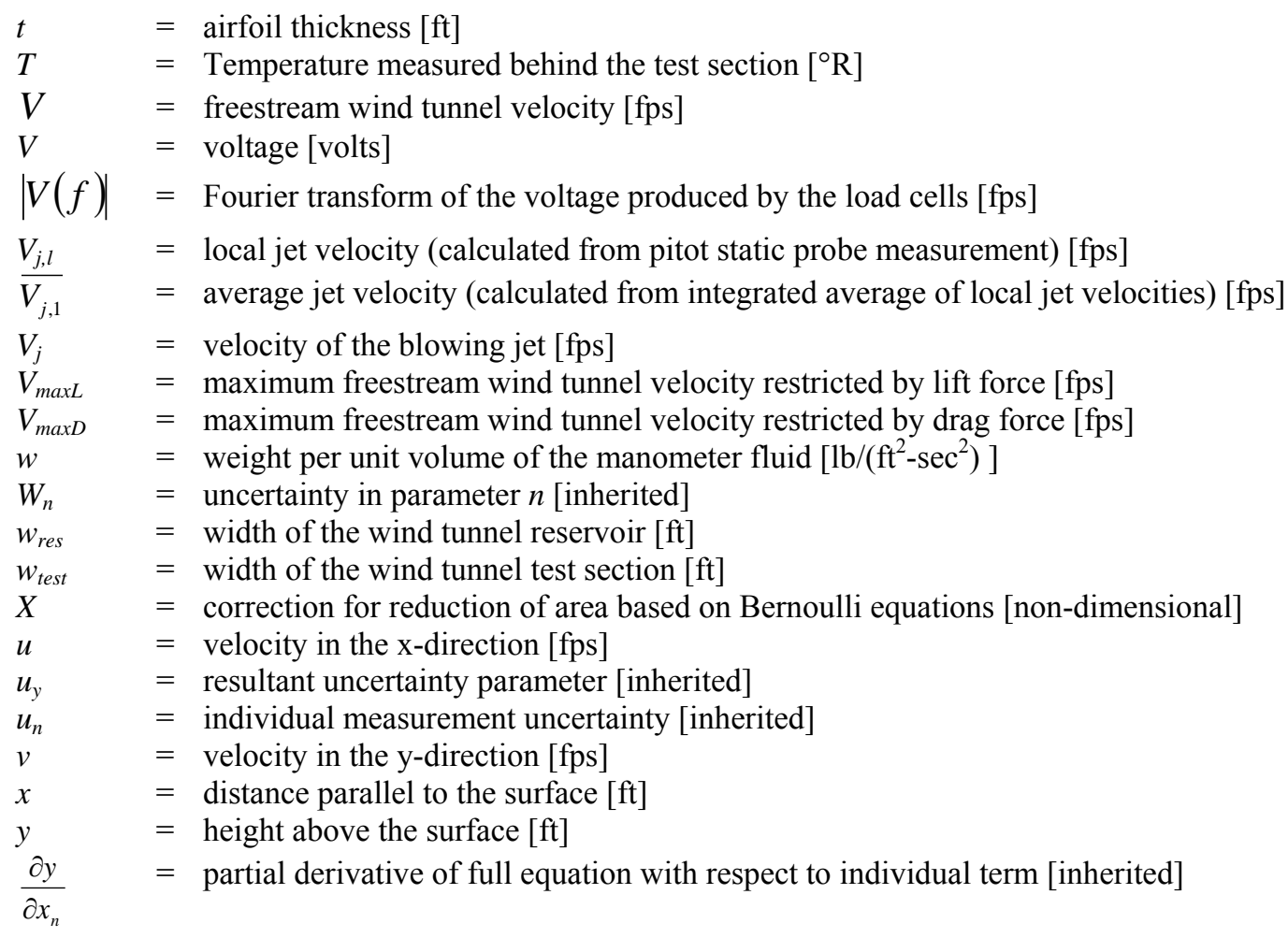

\section{Superscripts}

$=$ uncorrected value

\section{Subscripts}

$\begin{array}{ll}\mu & =\text { blowing parameter } \\ 0 & =\text { total } \\ \infty & =\text { freestream, atmospheric } \\ \mathrm{A} & =\text { applied } \\ \mathrm{B} & =\text { Blockage } \\ \mathrm{BD} & =\text { bottom drag } \\ \mathrm{BL} & =\text { bottom lift } \\ \mathrm{c} & =\text { constant } \\ \mathrm{cc} & =\text { circulation control } \\ \mathrm{d} & =\text { at plenum duct } \\ \mathrm{d} & =2 \text {-D drag } \\ \mathrm{D} & =\text { drag } \\ \mathrm{eff} & =\text { effective } \\ \mathrm{H}{ }_{2} \mathrm{O} & =\text { water } \\ \mathrm{j} & =\text { jet } \\ \mathrm{j}, \mathrm{l} & =\text { local at the jet } \\ \mathrm{l} & =2-\mathrm{D} \text { lift } \\ \mathrm{L} & =\text { lift } \\ \mathrm{LE} & =\text { leading edge } \\ \mathrm{m} & =\text { mass } \\ \mathrm{m} / 2 & =\text { half width of the jet } \\ \mathrm{max} & =\text { maximum value } \\ \mathrm{min} & =\text { minimum value } \\ \mathrm{model} & =\text { model }\end{array}$




$\begin{array}{ll}\mathrm{n} & =\text { varying parameter } \\ \mathrm{p} & =\text { pipe } \\ \mathrm{R} & =\text { read } \\ \mathrm{res} & =\text { reservoir } \\ \mathrm{s} & =\text { supply } \\ \mathrm{t} & =\text { throat } \\ \text { tare } & =\text { tare correction } \\ \mathrm{TD} & =\text { top drag } \\ \mathrm{TE} & =\text { trailing edge } \\ \text { test } & =\text { test section } \\ \mathrm{TL} & =\text { top lift } \\ \text { tunnel } & =\text { wind tunnel } \\ \mathrm{u} & =\text { uncorrected } \\ \mathrm{V} & =\text { venturi } \\ \mathrm{x} & =\text { varying parameter (usually varying pressure in psi) } \\ \mathrm{xx} & =\text { normal in } \mathrm{x} \text {-direction } \\ \mathrm{xy} & =\text { shear across } \mathrm{x} \text { and } \mathrm{y} \text {-direction } \\ \mathrm{yy} & =\text { normal in } \mathrm{y} \text {-direction }\end{array}$




\section{Chapter 1: Introduction}

A helicopter blade section has been designed that incorporates circulation controlled blowing slots for testing in the Closed-Loop Wind Tunnel at West Virginia University (Angle, 2008, Kweder, 2008). According to Johnson, 1980, "the primary limitation with many current [helicopter] designs is retreating blade stall, which at high speed produces an increase in rotor and control system loads and helicopter vibration, severe enough to limit the forward flight speed." Critzos, 1955, follows with the statement that higher forward flight speeds result in an increase of the "very high angle-of-attack region... and the forces contributed by this region constitute a greater part of the total rotor forces... Therefore, airfoil characteristics at very high angles of attack must be available in order to arrive at dependable performance estimates." This research investigated the subsonic reverse-flow two-dimensional aerodynamic characteristics of a 10:1 elliptical circulation controlled airfoil through stalling angles of attack and low blowing rates. The following section explains the need for this investigation in further detail.

When a helicopter rotor is in forward flight, the local velocities of the rotor change as the rotor rotates around the hub (around the azimuth angle). This creates an asymmetrical flow structure, as seen by Figure 1.1 where the advance ratio is the tip speed ratio, $\mu_{T}$, defined in Equation (1.1) as the ratio of the freestream velocity, $V_{\infty}$, (forward speed) divided by the rotor rotational speed, $\Omega$, multiplied by the overall rotor radius, $R$, as defined by Johnson, 1980. As the helicopter increases forward flight speed and the rotor speed is constant, the tip speed ratio increases. Johnson states that current helicopters achieve a maximum design tip speed ratio of approximately 0.3 to 0.4. These maximum ratios are limited by many factors, the greatest of which are the advancing rotor tip local velocity reaching transonic conditions and the retreating rotor inboard sections achieving reverse flow or blade stall conditions. At these extreme conditions, the rotor element is subject to high vibratory loads which can cause instabilities in the control system. Therefore, to achieve higher forward flight speeds, the transonic, blade stall, and reverse flow conditions need to be addressed.

$$
\mu_{T}=\frac{V_{\infty} \cos \alpha}{\Omega R}
$$




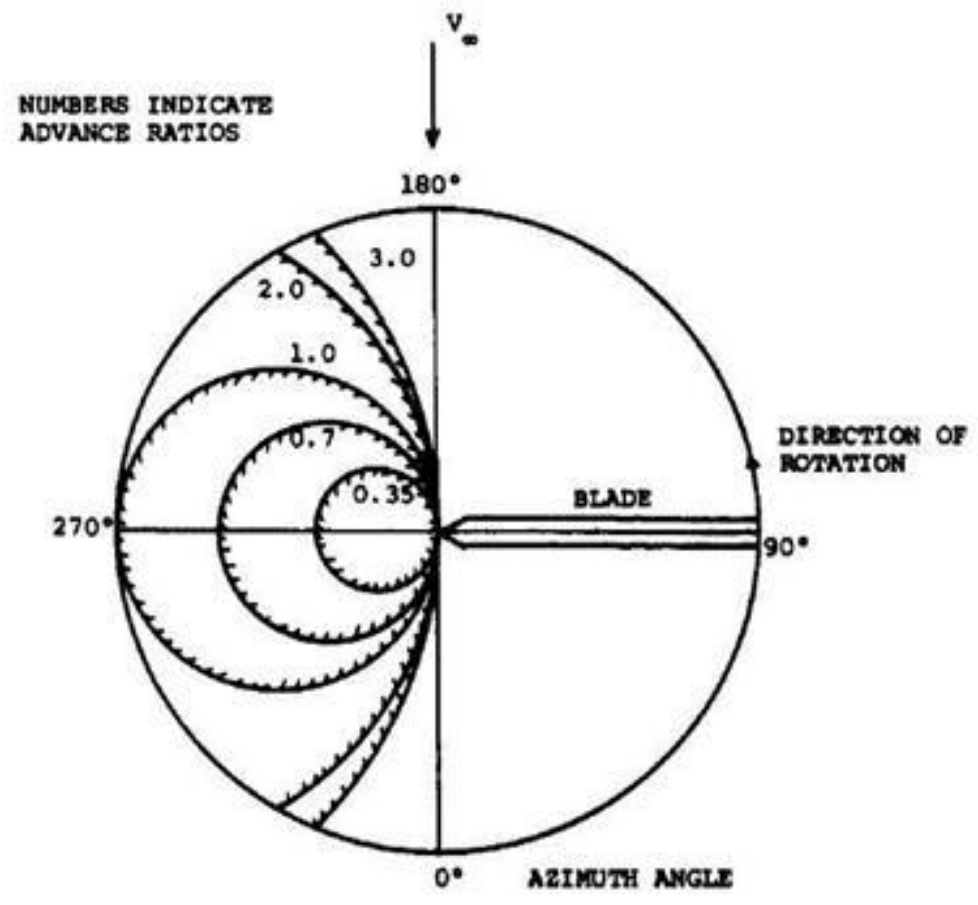

Figure 1.1: Growth of Reversed Flow Regions with Increasing Advance Ratios [Wood, 1981], Note: Reversed Flow Occurs Over the Portion of the Blade within the Operating Advance Ratio Circle

One method of reducing the transonic condition is to decrease the rotor speed. However, as the rotor speed is decreased, the tip speed ratio increases, causing a larger portion of the total flow structure for the rotor to be in reverse flow. This results in an asymmetric loading condition. The proposed circulation controlled rotor system potentially reduces this asymmetric loading condition by producing lift in the reverse flow regions and reducing blade stall. This is achieved by effectively changing the angle of attack of the blade as a function of the azimuth angle, the rotational position within the rotor plane. Conventionally, helicopters employ physical blade pitch change and flapping by rotor articulation to reduce this asymmetric loading, which highly increases the mechanical complexity. In contrast, the effective angle-of-attack can be changed by circulation control (blowing slots along the leading and trailing edges of the airfoil), eliminating the need for physical blade pitch change and rotor articulation.

Such a system's performance could be modeled by a modified version of pre-existing mathematical models and codes, which have been developed to simulate the complex rotor system of a conventional helicopter. Included in these codes are models which simulate the physical blade pitch change, flapping and lagging dynamics, and the structural responses to the forces and moments generated by the rotor blade. The blade element method is a common approach which divides the rotor into segments or elements in the spanwise direction. The elements are then treated as two-dimensional airfoils and the aerodynamic characteristics are evaluated at various local flow conditions, including transonic, stalled, yawed, and reverse flows. These aerodynamic characteristics, mainly the lift 
and drag coefficients through 360 degrees for angles of attack and Mach numbers from 0 (subsonic) to 0.84 (transonic), are needed for the programs' prediction of rotor performance characteristics.

The modified version of this program would include another parameter, the blowing coefficient (explained later), which defines the blowing rates for the circulation control. This parameter would be added to the look-up algorithms for the lift and drag coefficients and would allow the simulation of the circulation controlled rotor.

As a result, two-dimensional airfoil data for the aerodynamic characteristics based on Mach number, angleof-attack, and blowing coefficient is needed for a rotor performance simulation. Prior research has investigated these characteristics through small angles of attack and through transonic conditions for elliptical airfoils similar to the airfoil under investigation. However, a complete data set including reverse flow has not been provided.

This research investigated a portion of the two-dimensional aerodynamic characteristics, specifically the reverse-flow, two-dimensional aerodynamic characteristics of a 10:1 elliptical circulation controlled airfoil at subsonic conditions through stalling angles of attack and low blowing rates. 


\section{Chapter 2: Literature Review}

In order to understand the experimental and associated setup for this investigation, a review of the general concepts, definition, and evolution of circulation control is documented in this section. Also included in this section is a review of prior experimental setups.

\subsection{Circulation Control Review - General Concepts and Definition}

Circulation control is a highly efficient method for lift augmentation at low subsonic conditions (Novak, 1987). This type of control replaces a traditional mechanical flap or blown flap which has a sharp trailing edge with a fixed rounded or nearly rounded trailing edge. A jet blows tangentially along the trailing edge which "remains attached to the curved surface by creating a balance between the sub-ambient pressure and the centrifugal force." (Bertin, 2002). This jet flow entrains the flow around the wing, preventing separation. The flow then continues to follow the curve around the trailing edge (Cheesman, 1966, Englar, 1970, Mavis and Kirby, 1999). This effect known as the Coanda effect (as it was first studied by M. Henri Coanda) and moves the separation point to the lower side of the airfoil, hence increasing the circulation (and by default, the lift) around the airfoil (Abramson, 1979). A reproduction of this work can be found in Young and Zonars, 1950. Since the trailing edge is rounded, unlike the mechanical or blown flaps, high lift coefficients can be achieved without the penalty of large blowing momentum. A summarized Coanda effect explanation as proposed by Wood, 1981 is shown by Figure 2.1.

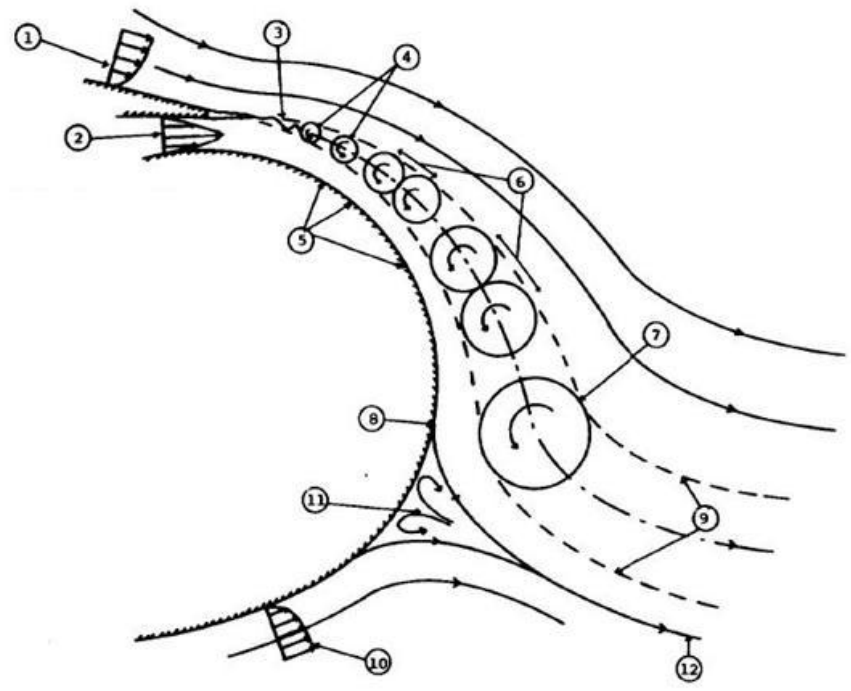

1. Upper surface boundary layer

2. Jet flow

3. Instability of the shed vortex sheet due to strong entrainment

4. Discrete vorticies formed - frequency dependant upon geometry and $\mathrm{cm}$

5. Reduced static presuure between vortices and wall - attachment

6. pairing process when adjacnt vortices touch

7. Limit of pairing process

8. Detachment due to limit of vortex stream

9. Shear layer limitations

10. Lower surface boundary layer

11. Separation bubble

12. Stagnation streamline

Figure 2.1: Proposed Coanda Effect Explanation [Wood, 1981] 
The Coanda effect can be used to generate one of three effects: Boundary Layer Control, Circulation Control, or a Jet flap effect. According to Wood, 1981 and others, the type of control or effect is determined by the augmentation ratio shown in Figure 2.2. The augmentation ratio is defined as the slope of the lift coefficient vs. blowing coefficient. The lift coefficient is defined by the lift force to freestream momentum ratio and the blowing coefficient is defined by the jet moment to freestream momentum ratio. These parameters are defined in more detail in Chapter 4. The lift augmentation ratio for a good circulation control airfoil is typically between 50 and 70 (Alexander and Anders, 2005).

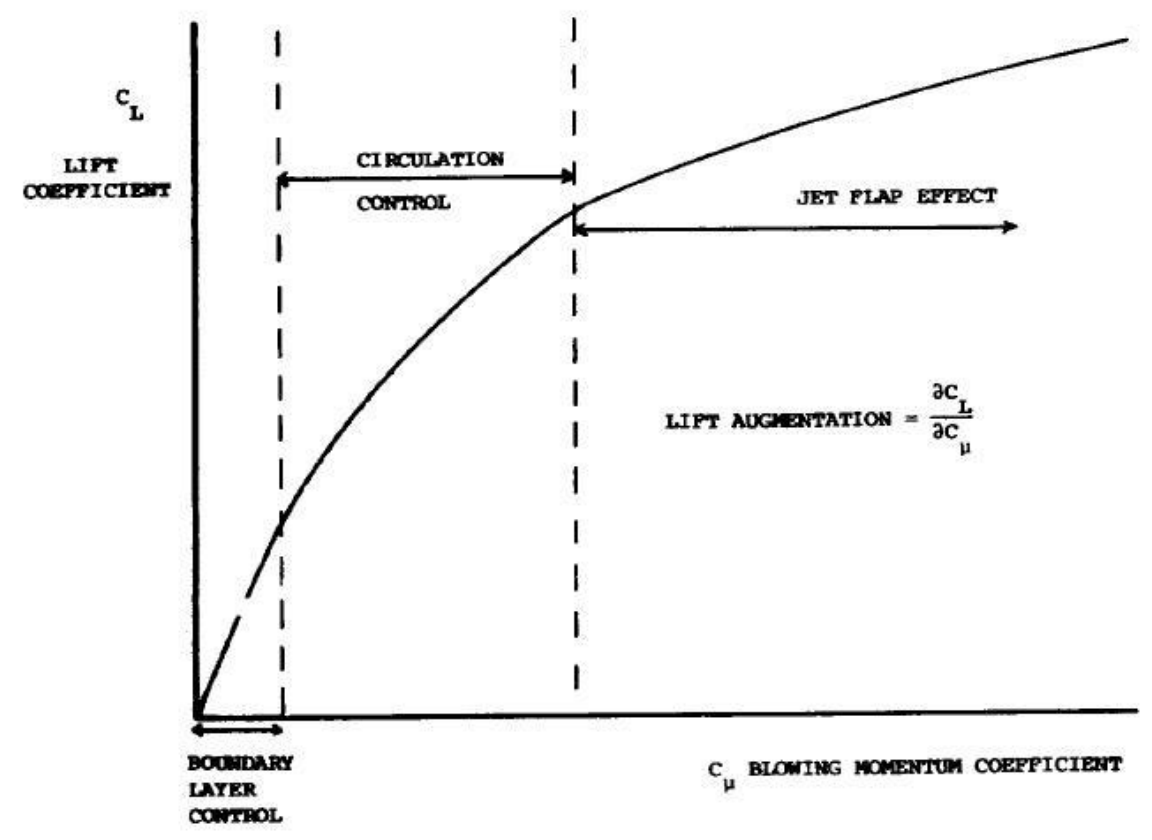

Figure 2.2: Performance Trends for a Circulation Controlled Aerofoil [Wood, 1981] 


\subsection{Circulation Control Evolution}

More specifically speaking, circulation control is achieved by ejecting a thin, high-momentum, turbulent jet tangentially along the wall of a convex surface. This complex phenomenon is a combination of several effects and its theoretical foundations have been built up through the experiments documented in this section. These experiments have investigated the consequences of plane jets, curved jets, and finally circulation control airfoils.

\subsubsection{Plane Jets}

A fundamental concept in the build-up of circulation control is a plane jet. This is defined by a jet which is blown in parallel, next to a solid surface or wall. An important parameter in plane wall jet experiments is the linear half velocity growth rate or velocity boundary layer. This boundary layer is defined by the region of the flow field where "the velocity of the fluid particles increases from a value of zero at the wall to the value that corresponds to the external frictionless flow outside the boundary layer (Bertin, 2002)." Compared to a similar free jet velocity boundary layer, this growth rate is $30 \%$ less, delaying separation, where the initiation of this boundary layer region may begin more than 50 slot widths downstream (Wood, 1981). This velocity boundary layer growth rate can be found in the review by Launder and Rodi, 1980 as Equation (2.1).

$$
\frac{d y_{m / 2}}{d x}=0.073 \pm 0.002
$$

Launder and Rodi, 1980 and Gartshore and Newman, 1969 provide a detailed summary of many plane wall jet experiments. Jet turbulence level, external flow, slot geometry, and longitudinal pressure gradients are some of the included topics in these experiments. Momentum balance discrepancies due to turbulence methods and assumptions are also discussed. These plane wall jet experiments provide a basis for the theoretical foundations, mainly the boundary layer growth and shear stress profiles that curved jets and circulation control airfoils are based upon.

\subsubsection{Curved Jets}

Increasing in complexity from the plane wall jet, a review of curved jet experiments provides an intermediate step in the understanding of the complex flow structure of circulation control. Ramaprian and Shivaprisad, 1977 and 1978, studied the boundary layer of a turbulent jet over curved surfaces and found that the wake in the outer portion of the boundary layer was increased with a convex surface, created by the centrifugal forces from the walls. Gibbs, 1975 found that, in addition to these wake changes, the shear stress distribution was also significantly altered, increasing in the outer regions of the boundary layer and decreasing in the inner regions. 
Newman, 1961 concluded that this curved jet flow structure is dependent on the blowing coefficient but not dependent on the Reynold's number (for Re $>9000$ ). He defines the boundary layer for a curved jet velocity profile with Equation (2.2), where the separation point is defined by Equation (2.3). This equation is derived from an angular maximum of 245 degrees, which was determined experimentally. Wilson and Goldstein, 1976 improved upon Newman's boundary layer equation with Equation (2.4). Dash and et Al, 1986 reviewed many of these experiments including Wilson and Goldstein, 1976, and Kind and Maull, 1968 and focused on correction techniques for the correlation of the experimental data. These corrections were based on turbulence models and gave a $\kappa-\varepsilon$ turbulence model correction for CFD analysis.

$$
\begin{gathered}
\frac{y_{m / 2}}{r \theta}=0.11\left(1+1.5 \frac{y_{m / 2}}{r}\right) \\
\theta=245-\frac{391 \frac{b}{r}}{1+1.125 \frac{b}{r}} \\
\frac{y_{m / 2}}{b}=0.0787\left(\frac{r \theta}{b}+6\right)\left[1+2.956\left(\frac{y_{m / 2}}{r}\right)-0.1559\left(\frac{y_{m / 2}}{r}\right)^{2}\right]
\end{gathered}
$$

From a theoretical perspective, Ambrosiani, 1971 derived the governing equations for two-dimensional incompressible flow for a curved wall jet. The continuity equation is shown in Equation (2.5), which is a function of density, time, radius of curvature, and distance above the surface as well as the $\mathrm{x}$ and $\mathrm{y}$-direction velocities.

$$
\frac{\partial \rho}{\partial t}+\frac{R}{R+y} \frac{\partial(\rho u)}{\partial x}+\frac{R}{R+y} \frac{\partial\left(\frac{R+y}{R} \rho v\right)}{\partial y}=0
$$

Ambrosiani, 1971 continued the derivation of the two-dimensional conservation equations with the $\mathrm{x}$ and $\mathrm{y}$ conservation of momentum equations shown in Equation (2.6) and Equation (2.7). These equations are additionally dependent on the normal stresses $\left(\tau_{x x}\right.$ and $\left.\tau_{y y}\right)$ and the shear stress $\left(\tau_{x y}\right)$ which are described by Equations $(2.8)$ through (2.10). These stresses are additionally dependent on the dynamic viscosity of the fluid.

$$
\begin{gathered}
\rho\left[\frac{\partial u}{\partial t}+\frac{R}{R+y} u \frac{\partial u}{\partial x}+v \frac{\partial v}{\partial y}+\frac{u v}{R+y}\right]+\frac{R}{R+y}\left(\frac{\partial p}{\partial x}-\frac{\partial \tau_{x x}}{\partial x}\right)-\frac{\partial \tau_{x y}}{\partial y}-\frac{2}{R+y} \tau_{x y}=0 \\
\rho\left[\frac{\partial v}{\partial t}+\frac{R}{R+y} u \frac{\partial v}{\partial x}+v \frac{\partial v}{\partial y}-\frac{u^{2}}{R+y}\right]+\frac{\partial p}{\partial y}-\frac{R}{R+y} \frac{\partial \tau_{x y}}{\partial x}-\frac{\partial \tau_{y y}}{\partial y}-\frac{1}{R+y}\left(\tau_{y y}-\tau_{x x}\right)=0 \\
\tau_{x x}=2 \mu\left[\frac{R}{R+y} \frac{\partial u}{\partial x}+\frac{v}{R+y}-\frac{1}{3}\left(\frac{R}{R+y} \frac{\partial u}{\partial x}+\frac{\partial v}{\partial y}+\frac{v}{R+y}\right)\right] \\
\tau_{y y}=2 \mu\left[\frac{\partial v}{\partial y}-\frac{1}{3}\left(\frac{R}{R+y} \frac{\partial u}{\partial x}+\frac{\partial v}{\partial y}+\frac{v}{R+y}\right)\right]
\end{gathered}
$$




$$
\tau_{x y}=\mu\left[\frac{R}{R+y} \frac{\partial v}{\partial x}+\frac{R+y}{R} \frac{\partial\left(\frac{R}{R+y} u\right)}{\partial y}\right]
$$

The experimental, numerical, and theoretical observations provide a glimpse into understanding the effect of a plane wall jet on curved surfaces. These findings later provided the interest in testing boundary layer control effects on airfoils with the promise of streamline alteration without physical shape change.

\subsubsection{Circulation Controlled Airfoils (Curved Jets in External Flows)}

Increasing the flow structure complexity, curved jets in external flows are the final step in understanding the evolution of circulation control. As previously mentioned, this flow structure (Coanda effect) is separated into boundary layer control, circulation control, and the jet flap effect. The focus here is on the experimentation of the circulation controlled realm, where the greatest aerodynamic efficiency is achieved (See Alexander and Anders, 2005).

Many experiments have been performed for circulation controlled airfoils in forward flow. Wood, 1981 is one such investigation where the subsonic aerodynamic characteristics of leading and trailing edge blowing on a 20 $\%$ thick elliptical airfoil were tested. The results from this experiment indicated general trends for a circulation controlled airfoil with leading and trailing edge blowing and were valid for circulation controlled airfoils based on the fact that:

1) the performance estimates for lift were in agreement with Kind and Maull, 1968 and are shown in Figure 2.3, where the effective incidence was the effective angle of attack,

2) the augmentation ratio was on the order of 60 , which is within the range of circulation controlled airfoils, as shown in Figure 2.2

3) the optimum height-to-chord ratio was approximately 0.002, which was in agreement with Englar and Williams, 1975.

Alexander and Anders, 2005 also investigated a circulation controlled airfoil in forward flow, with a 6\% symmetric circulation controlled airfoil and tested through transonic conditions with different trailing edge shapes, blowing slot heights, and blowing coefficients through various angles of attack. Figure 2.4 and Figure 2.5 show a sample of Alexander and Anders's results (2005). Alexander and Anders, 2005 found that at subsonic conditions, the data indicated that smaller Coanda surface and slot heights produced higher lift and pitching moments. In contrast, at transonic conditions, the larger Coanda surfaces and slot heights produced greater lift and pitching moments. The lift augmentation ratio of 27 for Alexander and Anders's (2005) experiment at low Mach numbers suggested that the model may be more of a Coanda jet flap than a circulation controlled airfoil. Further indications 
of transonic effects were presented by Alexander and Anders, 2005 but were not included here due to the nontransonic focus of this investigation.

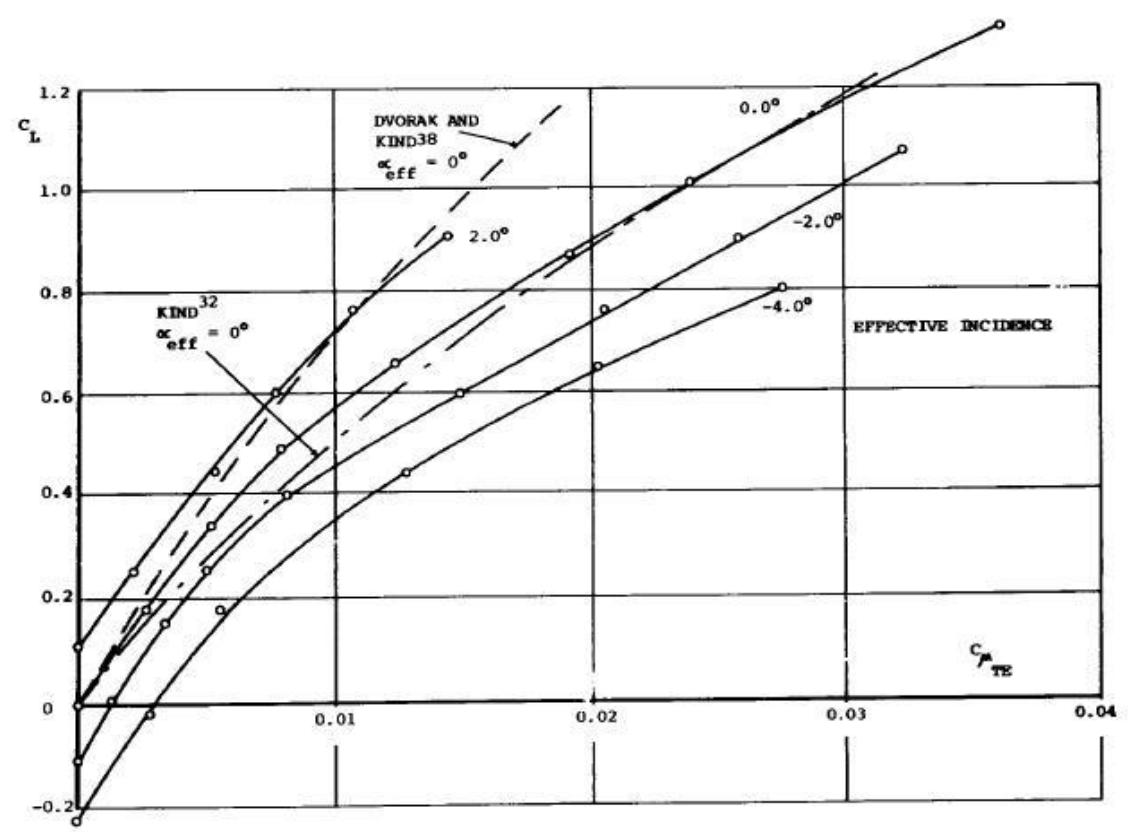

Figure 2.3: Lifting Performance of the Airfoil as a Function of Effective Incidence for Trailing Edge Blowing Only [Wood, 1981]

In spite of the extensive experimentation and analysis of a circulation controlled airfoil in forward flows, the investigation of a thin circulation controlled airfoil in reverse flow, similar to the current investigation, has not been found. The purposed circulation control in reverse flow is envisioned to create a much different flow structure, where adverse or reversed effects are likely. 


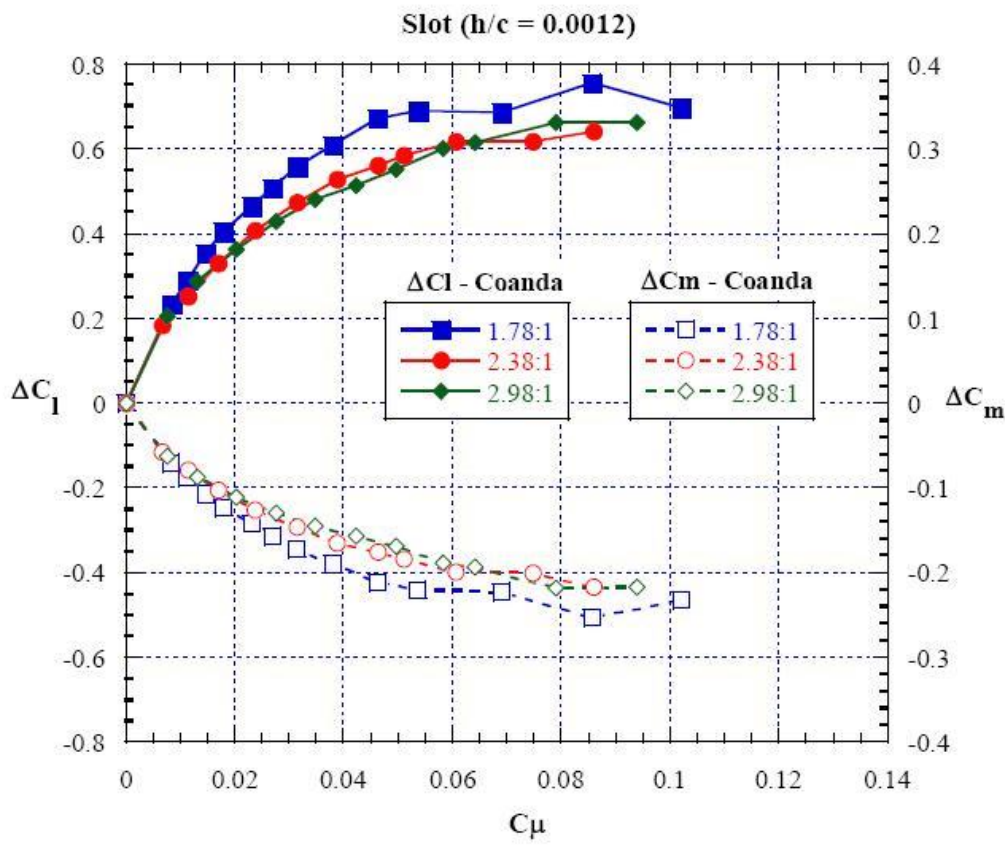

Figure 2.4: Change in lift coefficient and moment coefficient vs. blowing coefficient at $M=0.3, \alpha=+6$ deg [Alexander and Anders, 2005]

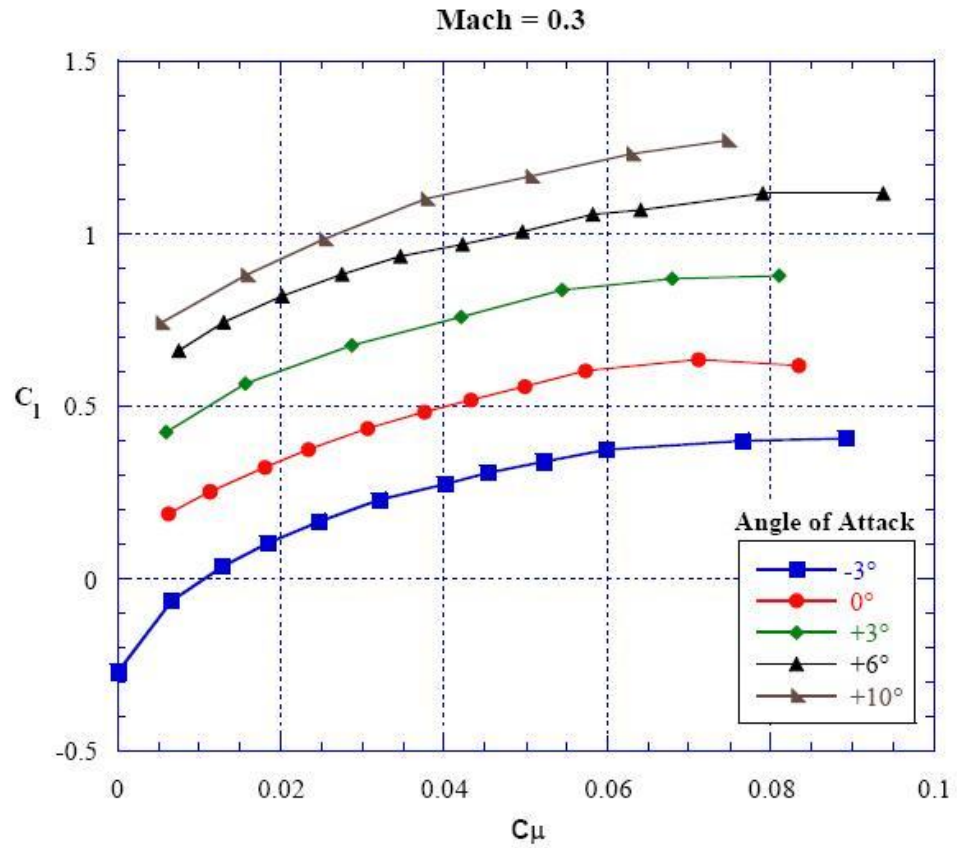

Figure 2.5: $C_{1}$ vs. $C_{\mu}$, angle of attack effect, upper slot blowing, Coanda (2.98:1), slot $(\mathrm{h} / \mathrm{c}=0.0012)$ [Alexander and Anders, 2005] 


\section{Chapter 3: Experimental Setup Review}

To obtain the greatest accuracy for this experimental investigation, a review of prior experimental setups was necessary. Specifically, this review examined and compared the construction materials, model geometries, measurement techniques, and correction methods from Wood, 1981, Rogers and Donnelly, 2004, Alexander and Anders, 2005, and Kweder, 2008. The experimental setup and model design for these circulation control experiments were very similar, and provided valuable insight on the design of the current setup.

\subsection{Model Construction Materials}

The construction of these airfoil models required high strength and the ability to finish the surfaces to a relatively low surface roughness. The most common material used for these models was aluminum due to its relatively low cost and ease of manufacturing. Rogers and Donnelly, 2004 used a brass Coanda surface in an attempt to increase smoothness, but the surface was easily scoured due to the material's softness.

\subsection{Model Geometry}

The model geometries for all the models used in these experiments were elliptical airfoils which are simple mathematically to reproduce for theoretical studies. Three major geometrical parameters of the models were studied in this review: chord length, span, and jet slot height.

The model chord length was determined by the chord length-to-tunnel height ratio. This ratio should be less than 0.3 for a CC airfoil (Englar and Williams, 1975), but the chord length should be as large as possible to obtain the high Reynold's numbers (Englar and Williams, 1975 and Barlow, Rae, and Pope, 1999). The chord length-totunnel height ratio ratio is important in reducing the wall interference effects in the wind tunnel.

The model span was typically determined by two recommended factors: The blockage ratio should be approximately 5\% (Barlow, Rae, and Pope, 1999) or 7\% with corrections (Allen, 1944) and the minimum height-towidth (aspect) ratio should be at least 1.5 (Barlow, Rae, and Pope, 1999) or 2 (Englar and Williams, 1975). The blockage ratio in percent is defined by Equation (3.1), where the area of the model is defined by Equation (3.2). As the angle of attack increases, the blockage ratio of the model increases and wall effects increase. For example, if the angle of attack range desired is large, the span or chord design may be reduced to stay within the recommended $7 \%$ blockage ratio.

$$
\begin{aligned}
& B R=A_{\text {model }} / A_{\text {tunnel }} * 100 \\
& A_{\text {model }}=(c \sin \alpha+t \cos \alpha) b
\end{aligned}
$$


Finally, the jet slot height is calculated from the jet height-to-chord ratio which is recommended to be as small as possible without slot expansion from internal pressurization (Englar and Williams, 1975). This recommended small height comes from the fact that the jet exit velocity is increased through the reduction of the slot area. This velocity increase directly increases the blowing momentum. Most experiments reviewed have a jet heightto-chord ratio within the range of 0.001 to 0.002 . Finally, the thickness-to-chord ratio of the model is usually chosen based the ability to house instrumentation and provide a consistent air supply for the jet.

\subsection{Measurement and Calculation Techniques for Important Non- Dimensional Parameters}

Now that the model geometry has been reviewed and compared, previous calculation methods for important non-dimensional parameters and their associated measurement techniques become the focus of this section. The main parameters studied in this review are the non-dimensional coefficients for lift, drag, and blowing, and are described further in Chapter 4.

The coefficients of lift and drag are functions of the lift or drag force, dynamic pressure, and model area. Since the calculation of the dynamic pressure and the model area is relatively straight forward and standardized, the focus of this review is the lift and drag forces for the calculation of the lift and drag coefficients.

The lift force is determined by either integrating the static pressure measurements (surface pressure taps) over the chord length or by the use of a force balance. In the case of Rogers and Donnelly, 2004 and the current setup, a force balance was used that employs a combination of load cells. However, the pressure integration method is the preferred (and typical) method of lift force calculation due to the reduction of three-dimensional effects (Englar and Williams, 1975).

Drag force calculation is similar to the lift calculation in a force balance method, but integrates the static pressure from a wake rake in the pressure integration method. Drag force calculated from a force balance has many associated problems and is "essentially useless" as discussed in Englar and Williams, 1975. Thus, the wake rake pressure integration (a.k.a. velocity deficit) method is preferred.

Once these experimenters chose a method of instrumentation for the lift and drag coefficients, the decision of whether or not to correct for wall tunnel interference was made. Wood, 1981 incorporated a simple blockage correction to the freestream velocity as described in Pankhurst, 1952 and defined by Equation (3.3) where the blockage factor, $\varepsilon_{B}$, is defined by Equation (3.4). The parameter $\lambda$ in the blockage factor equation is defined by Equation (3.5). The parameter $\tau_{B}$ is equal to 0.822 for a closed wind tunnel. Alexander and Anders, 2005 and Rogers and Donnelly, 2004 chose not to correct for wall tunnel interference. The current model used corrections from the Allen and Vincenti method (Allen, 1949), which is also used by Abbot and Von Doenhoff, 1945 and is described further in Chapter 4. 


$$
\begin{gathered}
V=V_{u}\left(1+\varepsilon_{B}\right) \\
\varepsilon_{B}=\tau_{B} \lambda\left(\frac{t}{h}\right)^{2} \\
\lambda=\frac{1}{2}\left(1+\frac{c}{t}\right)
\end{gathered}
$$

The final coefficient to be reviewed for measurement technique and calculation is the blowing coefficient. This parameter is a function of the mass flow and velocity of the jet, freestream dynamic pressure, and model area. The important parameters are the mass flow and velocity of the jet as per the discussion above for the freestream dynamic pressure and model area.

Mass flow as well as jet velocity is commonly measured by an isentropic expansion method (Englar and Williams, 1975). This method assumes isentropic expansion from the model plenums to the free stream and requires both total pressure and total temperature for proper calculation. The equation used for the calculation of mass flow is dependent on whether the flow is subcritical (subsonic) or critical (choked or sonic) as described by Equation (3.6) and Equation (3.7), respectively (Englar and Williams, 1975).

$$
\begin{gathered}
\dot{m}_{j}=A_{t} p_{0} \sqrt{\frac{2 \gamma}{(\gamma-1) R T_{0}}\left(\left(\frac{p}{p_{0}}\right)^{\frac{2}{\gamma}}-\left(\frac{p}{p_{0}}\right)^{\frac{\gamma+1}{\gamma}}\right)} \\
\dot{m}_{j}=A_{t} p_{0} \sqrt{\frac{\gamma g_{c}}{R T_{0}}}\left(\frac{2}{\gamma+1}\right)^{\frac{\gamma+1}{2(\gamma-1)}}
\end{gathered}
$$

Both Wood, 1981 and Alexander and Anders, 2005 used the isentropic expansion to determine mass flow. However, the measurement locations and techniques differed. Alexander and Anders, 2005 used a system of multiple critical venturis where the total pressure was measured upstream of the venturi and the static pressure and total temperature was measured at the throat of the venturi as shown in Figure 3.1. Wood, 1981 similarly positioned the pressure and temperature measurements but used an orifice plate in place of a venturi and measured the pressure drop across the orifice. Wood, 1981 also calculated the mass flow by substituting the jet exit area as the area of the throat and the plenum duct pressure as the total pressure in Equations (3.6) and (3.7). 


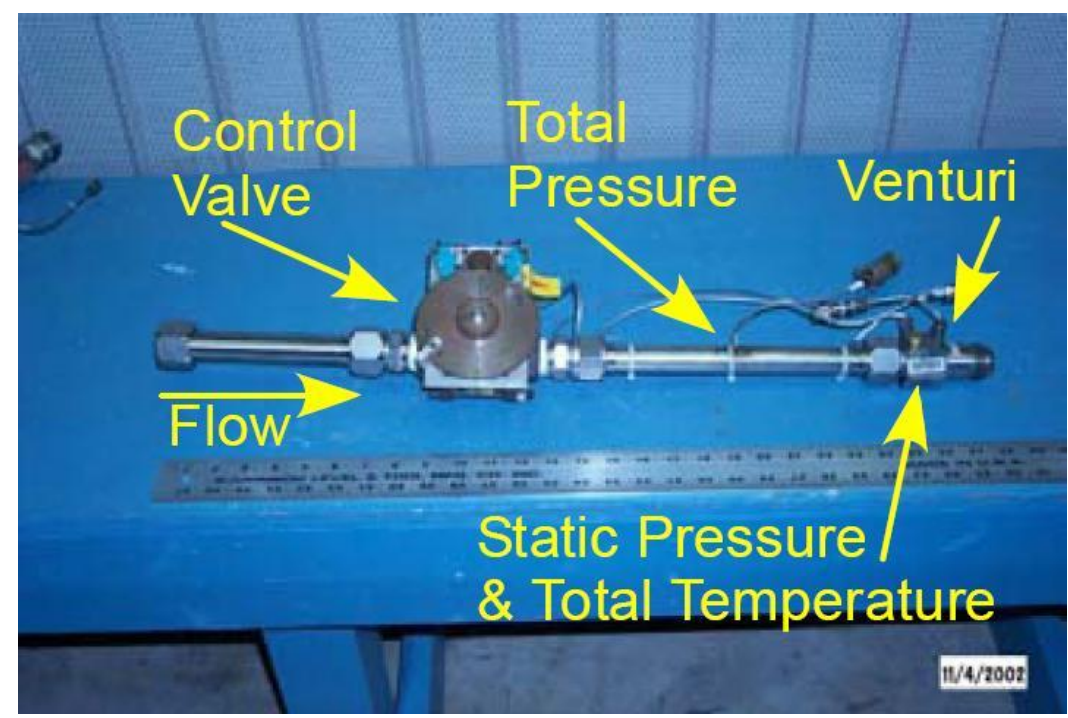

Figure 3.1: Mass Flow Measurement, Critical Venturi System [Alexander and Anders, 2005]

For determining jet exit velocity, Alexander and Anders, Wood, and Rogers and Donnelly used the isentropic expansion method described by Equation (3.8). In addition to this technique, Wood also used the substitution mentioned above and a hot-wire anemometer to determine the velocity of the jet and compared all three measurement methods in Figure 3.2. It is easily seen in this figure that all three methods were in relatively good correlation.

$$
V_{j}=\sqrt{2 R T_{d} \frac{\gamma}{(\gamma-1)}\left(1-{\frac{p}{p_{d}}}^{\frac{\gamma-1}{\gamma}}\right)}
$$

Table 3.1 shows the summary of the previous circulation control experimental setup and measurement techniques mentioned and a comparison to the current experimental setup. 


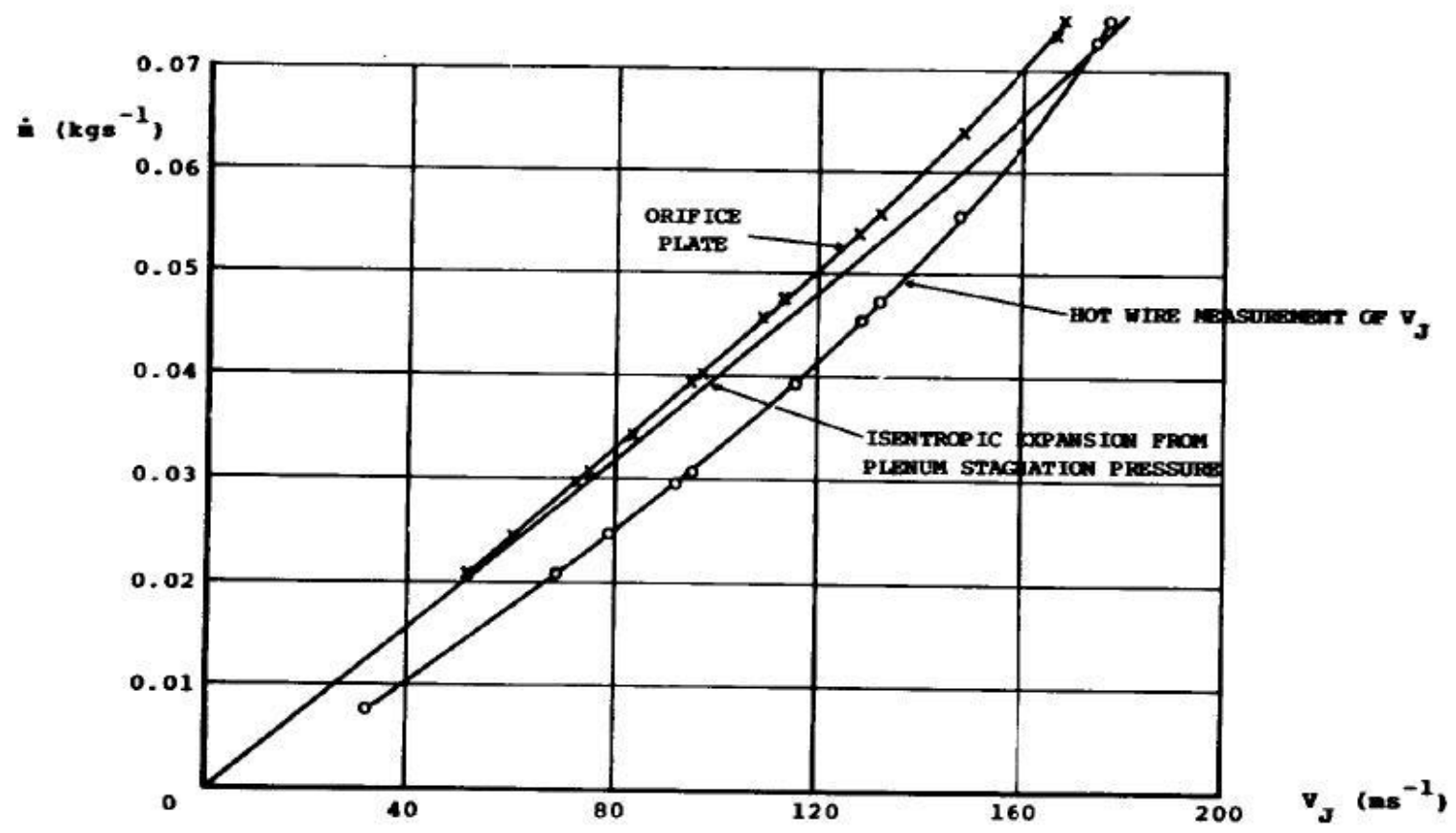

Figure 3.2: Estimation of Jet Velocity by Three Methods [Wood, 1981] 
Table 3.1: Experimental Setup Review and Comparison

\begin{tabular}{|c|c|c|c|c|}
\hline Experiment Reference & Wood, 1981 & $\begin{array}{c}\text { Rogers and } \\
\text { Donnelly, } 2004\end{array}$ & $\begin{array}{l}\text { Alexander and } \\
\text { Anders, } 2005\end{array}$ & $\begin{array}{l}\text { Kweder, } 2008 \text { and } \\
\text { Current }\end{array}$ \\
\hline Model Construction / Design & $\begin{array}{l}\text { Aluminum Skin } \\
\text { around Ribs }\end{array}$ & $\begin{array}{l}\text { Solid Aluminum, } \\
\text { Brass Coanda } \\
\text { surface }\end{array}$ & $\begin{array}{l}\text { Aluminum Skin, } \\
\text { Standoff Supports }\end{array}$ & $\begin{array}{l}\text { Aluminum Skin } \\
\text { around Ribs }\end{array}$ \\
\hline Airfoil Shape (elliptical, etc) & Elliptical & Elliptical & Elliptical & Elliptical \\
\hline Chord Length, $c$ (approx.) & $24 "$ & $24 "$ & $30^{\prime \prime}$ & $12^{\prime \prime}$ \\
\hline Model Span, $b$ & $24 "$ & 24" (average) & $60 "$ & $31 "$ \\
\hline Aspect Ratio, $b / c$ & 1 & 1 & 2 & 2.62 \\
\hline Chord to Tunnel Height Ratio, $c / h$ & 0.390 & 0.200 & 0.156 & 0.369 \\
\hline Jet Height-to-Chord Ratio, $h_{j} / c$ & 0.0009 & 0.0019 & $\begin{array}{l}0.0007,0.0012 \\
0.0020,0.0026\end{array}$ & $\begin{array}{l}\text { Trailing }=0.0021 \\
\text { Leading }=0.0010\end{array}$ \\
\hline Thickness-to-Chord Ratio, $t / c \%$ & 20 & 20 & 6 & 10 \\
\hline $\begin{array}{l}\text { Leading Edge/Trailing Edge Dual } \\
\text { Blowing }\end{array}$ & Yes & No & No & Yes \\
\hline $\begin{array}{l}\text { Upper/Lower Surface Dual } \\
\text { Blowing }\end{array}$ & No & Yes & Yes & Yes \\
\hline Lift Force Calculation & Pressure Integration & Load Cells & Pressure Integration & Load Cells \\
\hline Drag Force Calculation & $\begin{array}{c}\text { Wake Rake } \\
\text { Pressure Integration }\end{array}$ & Load Cells & $\begin{array}{c}\text { Wake Rake } \\
\text { Pressure Integration }\end{array}$ & Load Cells \\
\hline Trailing Edge Shape & round, $\mathrm{R}=2.0^{\prime \prime}$ & round, $\mathrm{R}=0.8^{\prime \prime}$ & $\begin{array}{l}\text { elliptical, 1.78:1, } \\
\text { 2.38:1, 2.98:1 }\end{array}$ & round, $\mathrm{R}=0.4^{\prime \prime}$ \\
\hline Internal Screens & No & Yes & Yes & Yes \\
\hline Wind Tunnel & $\begin{array}{l}\text { Bath University } \\
\text { Low Speed }\end{array}$ & $\begin{array}{c}\text { NSWCCD } \\
\text { Cavitation Channel }\end{array}$ & $\begin{array}{l}\text { Langley Transonic } \\
\text { Dynamics Tunnel }\end{array}$ & WVU Low-Speed \\
\hline Tunnel Type & Closed Air & Water & Closed Air & Closed Air \\
\hline Test Section Size & $7 \mathrm{ft} \times 5 \mathrm{ft}$ & $10 \mathrm{ft} \times 10 \mathrm{ft}$ & $16 \mathrm{ft} \times 16 \mathrm{ft}$ & $3.75 \mathrm{ft} \times 2.67 \mathrm{ft}$ \\
\hline Mach Numbers Tested & 0.093 & 0.0002 & $0.3,0.7,0.8,0.84$ & $0.073,0.109$ \\
\hline Velocity Measurement & Tunnel Head & Unknown & Unknown & Tunnel Head \\
\hline Turbulence Modeling & Yes & No & No & No \\
\hline Angle-of-Attack Test Range & -7.5 to +7.5 & -40 to +40 & -3 to +10 & $\begin{array}{c}-16 \text { to } 16,164 \text { to } \\
196 \text { (current) }\end{array}$ \\
\hline $\begin{array}{l}\text { Blockage Factor, Wall Interference } \\
\text { Corrections }\end{array}$ & Pankhurst & No & No & No, Allen (current) \\
\hline Reynold's Number at low-speed & $1.3 \times 10^{6}$ & $2.1 \times 10^{6}$ & $9.0 \times 10^{5}$ to $1.4 \times 10^{6}$ & $4.4 \times 10^{5}$ to $7.1 \times 10^{5}$ \\
\hline Re Number-to-Chord Ratio, Re/c & $6.5 \times 10^{5}$ & $1.05 \times 10^{6}$ & $3.6 \times 10^{5}$ to $5.5 \times 10^{5}$ & $4.4 \times 10^{5}$ to $7.1 \times 10^{5}$ \\
\hline Blowing Supply & Air & Water & Air & Air \\
\hline Mass Flow Measurement & $\begin{array}{c}\text { Orifice Plate by } \\
\text { Model Plenum } \\
\text { Stagnation Pressure }\end{array}$ & $\begin{array}{l}\text { Precision Turbine } \\
\text { Flowmeter }\end{array}$ & $\begin{array}{c}\text { Pressure and } \\
\text { Temperature at } \\
\text { Critical Venturis } \\
\end{array}$ & $\begin{array}{c}\text { Flowmeter and } \\
\text { Total Pressure and } \\
\text { Temperature in pipe }\end{array}$ \\
\hline Jet Velocity Calculation & $\begin{array}{c}\text { Orifice Plate, Hot- } \\
\text { Wire, Isentropic } \\
\text { Exp. }\end{array}$ & $\begin{array}{l}\text { Simplified } \\
\text { Isentropic Exp. }\end{array}$ & Isentropic Exp. & $\begin{array}{l}\text { Pitot Static Probe } \\
\text { Experiment }\end{array}$ \\
\hline $\begin{array}{l}\text { Blowing Coefficient Testing } \\
\text { Range }\end{array}$ & [0 to 0.04$]$ & {$[0$ to 0.60$]$} & [0 to 0.40$]$ & [0 to 0.014$]$ \\
\hline
\end{tabular}




\section{Chapter 4: Methodology}

This section decribes the reasonings behind the selection of the physical model, facilities, and instrumentation as well as the methodology and correction methods used in the calculation of the important aerodynamic parameters.

\subsection{Facilities, Model, and Instrumentation Setup}

Once the prior experiments were examined and compared, a model design and instrumentation setup was chosen. This section describes the model design, equipment setup, facilities used, and the accompanying methodology for obtaining results in this investigation. The setup chosen was similar to the experiments performed by Angle, 2008 and Kweder, 2008, with the exception that the model (designed by Angle) is installed in reverse.

\subsubsection{Wind Tunnel Background and Instrumentation}

The first and most significant experimental decision that was made was choosing the facility for testing. Of the available facilities, the West Virginia University Closed-Loop Wind Tunnel was chosen mainly due to the fact that it could house the largest model possible and obtain high Reynold's numbers at low speeds, similarly to previous circulation control experiments.

This tunnel is a low-speed wind tunnel where the test section of the tunnel has a rectangular (corners have a 45 degree camfer) 32 " x 45 " cross section that can attain a freestream velocity of approximately $175 \mathrm{ft} / \mathrm{s}$ by pitch control of a constant speed propeller.

The instrumentation for many of the wind tunnel parameters in this wind tunnel was similar to the prior experiments by Angle, 2008 and Kweder, 2008. The tunnel head in the tunnel was measured directly from the monitoring system, which was correlated to the freestream velocity. The absolute (atmospheric) pressure was read from an absolute pressure sensor and the temperature of the air inside the tunnel was read from a spring scale thermometer needle gauge. Both the temperature and pressure were used to calculate the density and dynamic viscosity, and the geometry of the tunnel was used to provide velocity corrections. 


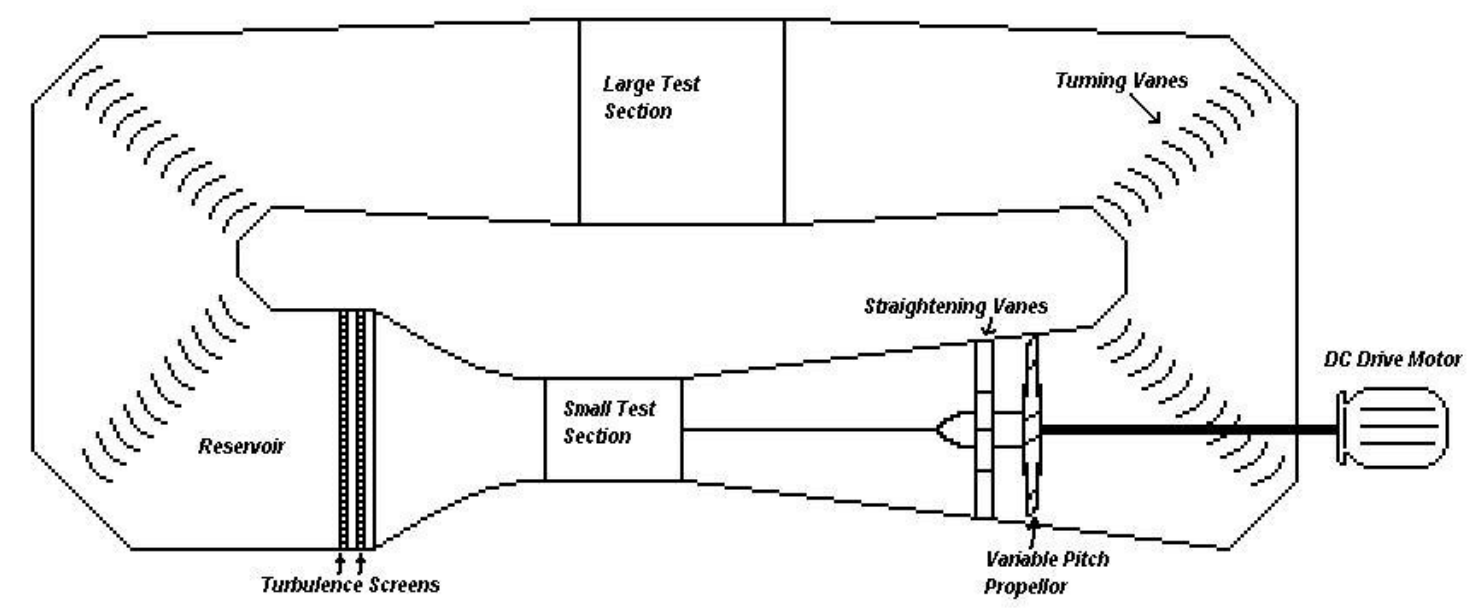

Figure 4.1: WVU Closed Loop Wind Tunnel (Not to scale)

Forces on the model were measured by reading the voltage change due to strain in S-beam type load cells connected to a National Instruments based data acquisition system. The load cells were attached at both the bottom and top of the vertically-mounted model, oriented in the lift and drag directions, and were independent of model angle-of-attack. Pressures could be measured by means of static ports in the model; however this functionality was not utilized for this study.

\subsubsection{Blade Segment Model (2-D Airfoil)}

The physical size of the wind tunnel test section had a significant impact on the design of the model. Since two-dimensional airfoil data is commonly used in many design schemes, including existing helicopter simulation programs to perform rotor performance evaluations, the model to be investigated was chosen by Angle to span the tunnel cross section to allow the airfoil to be treated as a two-dimensional, infinite span wing. This idea is congruent with many previous experiments (see Englar and Williams, 1975 and Wood, 1981). The model was then chosen by Angle to be mounted vertically to provide the largest chord (for the desirable largest Reynold's number) and the lowest chord-to-tunnel-height ratio (described in section Chapter 3: and in Englar and Williams, 1975). In this configuration, the angle of attack could be varied between 154 and 196 degrees without exceeding the 7\% blockage limit discussed earlier.

Once the span and chord length were chosen, the model geometry was selected by Angle to be a 10:1 (10\% thick) elliptical airfoil with a slightly modified leading and trailing edge. This airfoil selection was based on the results of previous experiments including minimizing the thickness to the amount of height needed to house the internal instrumentation. The trailing edge was modified to create a round Coanda surface which gave the airfoil a slight virtual camber. The leading and trailing edge also included blowing slots, which were supplied with air 
through the internal pressurization of the model and were controlled by turning cams which allowed for active circulation control. However, in the configuration under investigation, active opening was not required. Therefore, the slots were set in the desired configuration. The 3-D CAD representation of the entire model with endplates is shown in Figure 4.2. The cross-section of the airfoil model with the leading and trailing edge cams can be seen in Figure 4.3. The leading edge cam is colored blue and positioned on the right in Figure 4.3. The trailing edge cam is positioned on the left and colored red in the same figure.

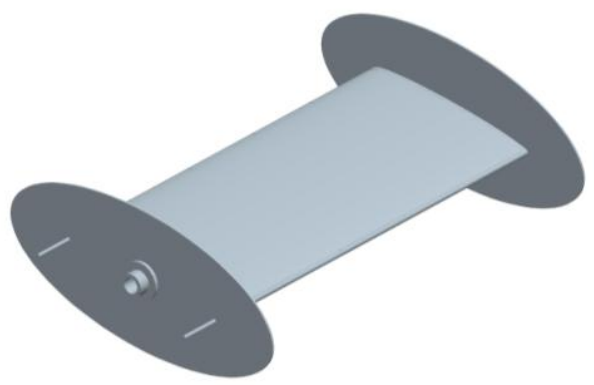

Figure 4.2: CAD Drawing for Proof of Concept Model of a Helicopter Blade with Circulation Control [Angle, 2006]

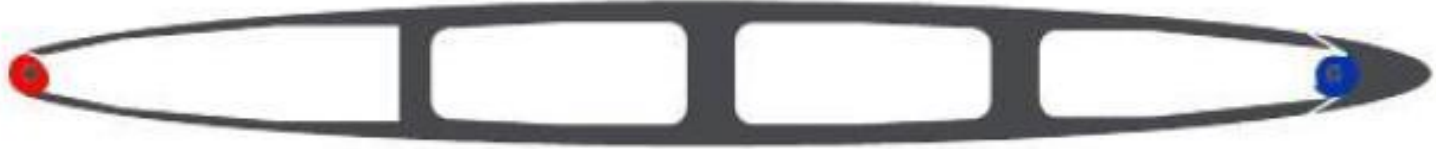

Figure 4.3: 2-D Cross Section of Entire Airfoil [Angle, 2008]

A single $20 \%$ porous wall was installed inside the model to provide plenum pressure equalization. This decision was based on model internal wall configuration and porosity test results similar to Alexander and Anders, 2005. The model was also designed to incorporate static pressure taps along the midspan of the model. However, after the model was constructed, the author of this document found that the number of taps on the upper and lower surface was inadequate (Englar and Williams, 1975) for proper application of the pressure integration method described in Alexander and Anders, 2005 and Wood, 1981. Figure 4.4 and Figure 4.5 show the internal structure of the model including pressure taps and the porous walls. 


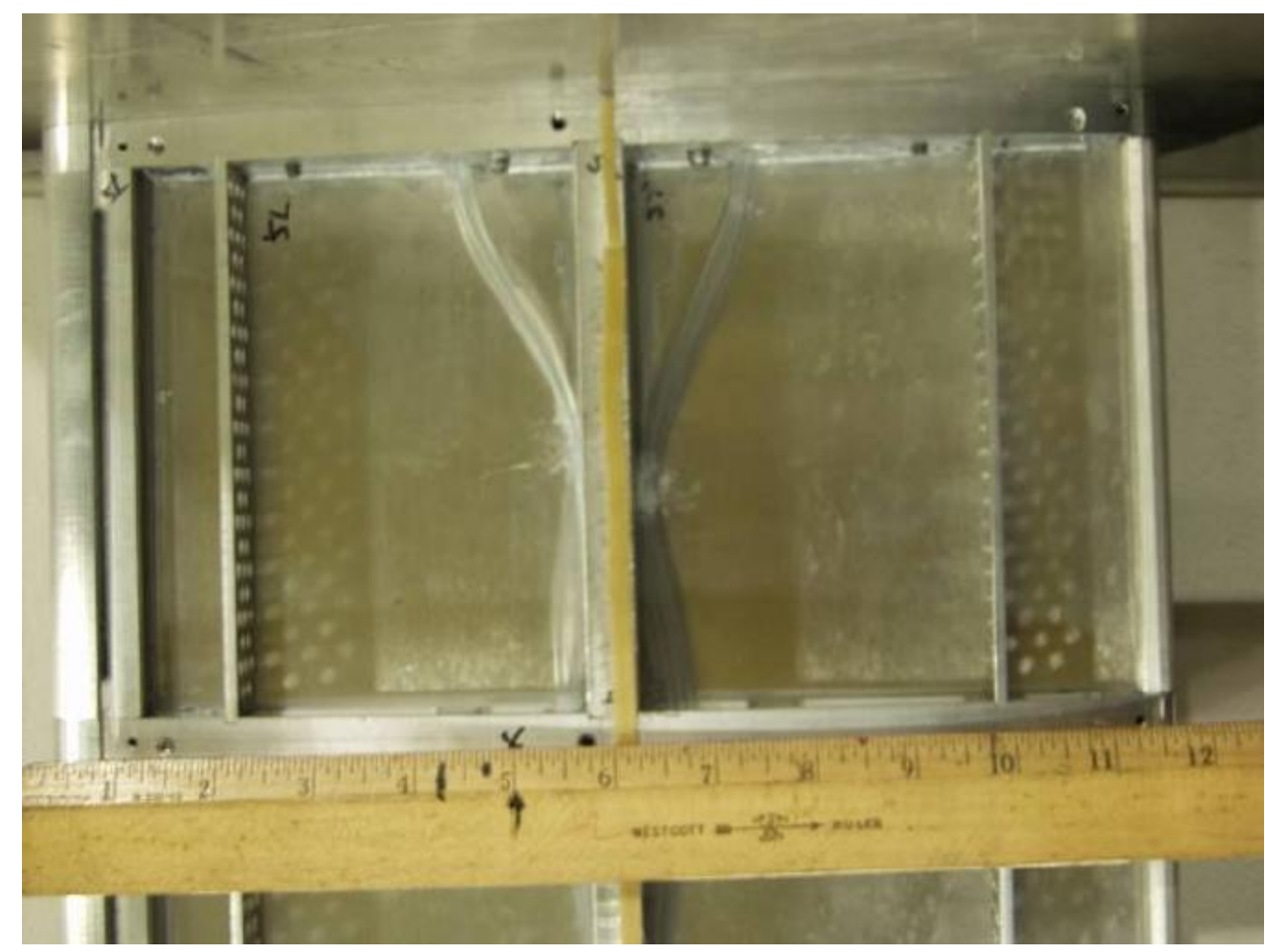

Figure 4.4: Internal Structure of the Blade Segment [Angle, 2008]

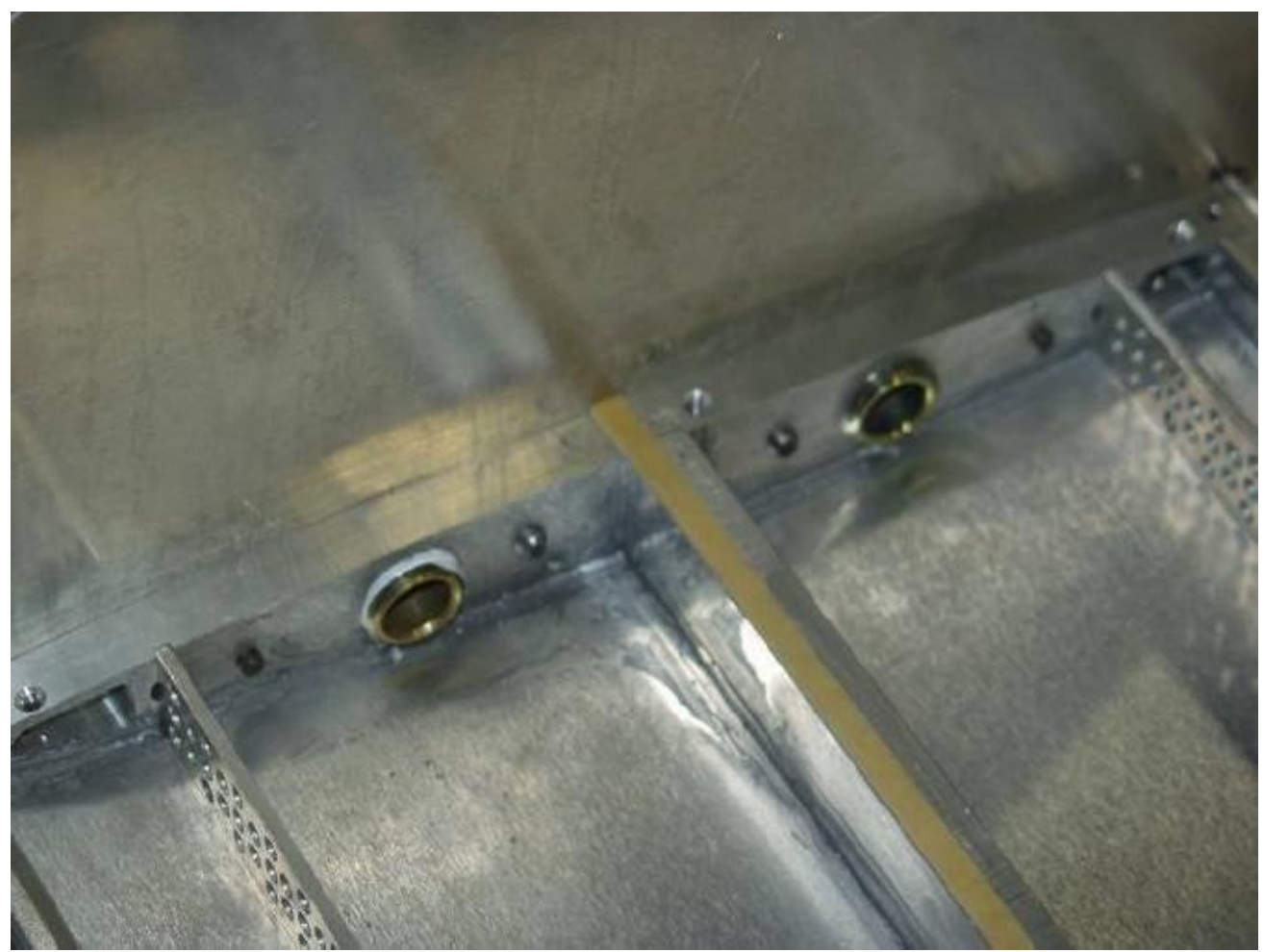

Figure 4.5: Internal Ports and Porous Walls of the Blade Element [Angle, 2008] 


\subsubsection{Wind Tunnel Test Stand Description}

Once the model was created, a suitable test stand was designed to mount the model and its associated instrumentation. The test stand was designed and created exclusively for the investigation of this model, and incorporated a large frame bolted to the concrete floor, made primarily from 4" x 8 " x 0.25 " aluminum I-beams and C-channels welded together as shown in Figure 4.6. The airfoil model sting is 31.5" tall, positioned vertically, with a small gap in the end plates to reduce vibrations created from contact with the wind tunnel test section.

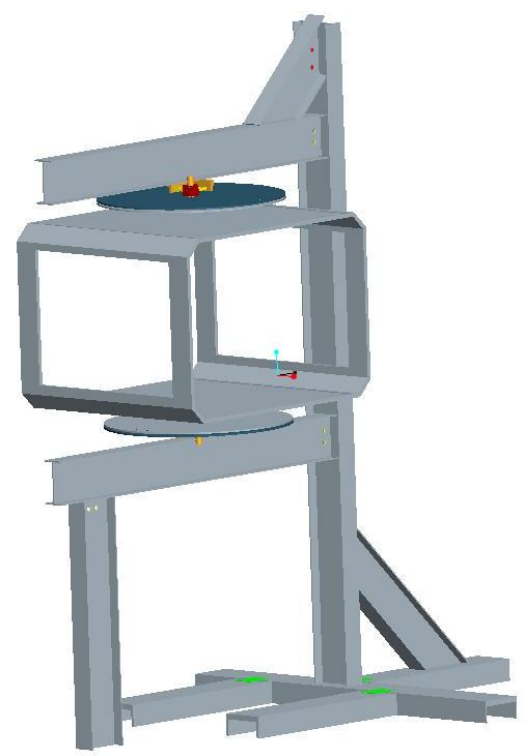

Figure 4.6: Test Section and Stand Design in as Developed in Pro-Engineer [Lyons, 2008]

A pair of rotatable 3/32" thick aluminum plates provided angle-of-attack variation. The outside plates were fixed and attached to the load cells while the inner plates were moveable and attached to the model. A $1 / 4$ " diameter steel pin was used to restrict rotation. The angle of attack could be varied from -180 to 180 in various increments. However, in this experiment, the angle of attack ranged from 164 to 196 degrees in 2 degree increments.

Four load cells were used in determining forces. Two one-dimensional Omega LC101-25 S-beam type load cells were placed at each hub of the rotating assembly (above and below the test section) as shown in Figure 4.7. 


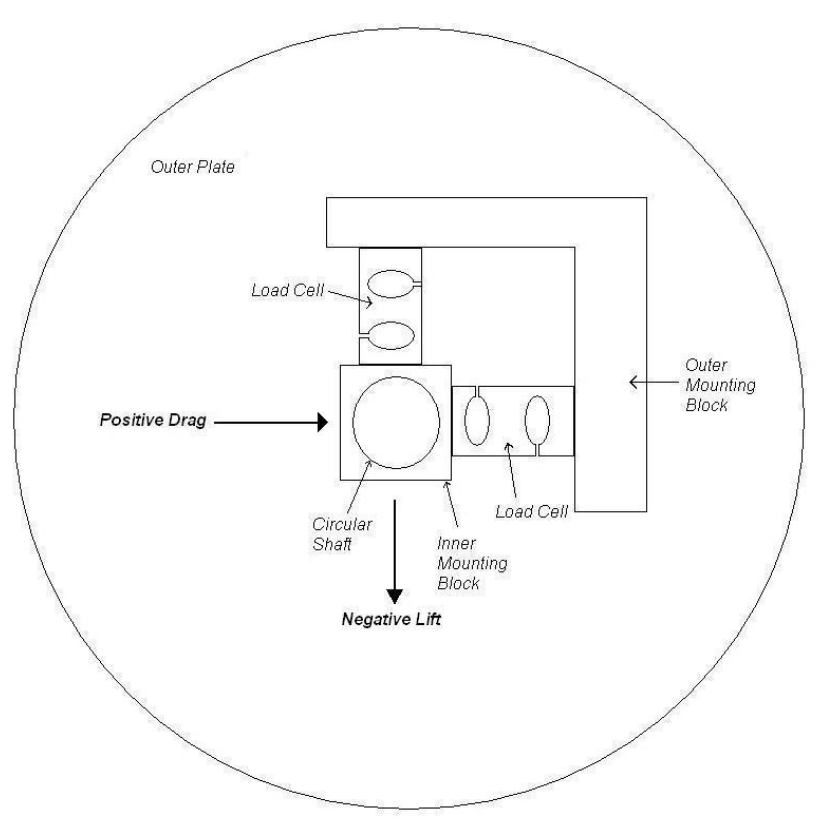

Figure 4.7: Rotating Assembly and Load Cell Setup

\subsubsection{Circulation Control Air Supply System}

The supply air system for the circulation controlled blowing was attached. Working backwards from the blowing slots, the circulation control blowing was supplied by pressurized chambers (plenums) within the airfoil. These chambers were connected by fittings to the supply lines which were controlled via pressure regulators and gauges, and were fed by a large pressurized tank. A summarized diagram of the air supply system leading up to the model can be seen in Figure 4.8. The tank was pressurized by a three-cylinder electric air compressor.

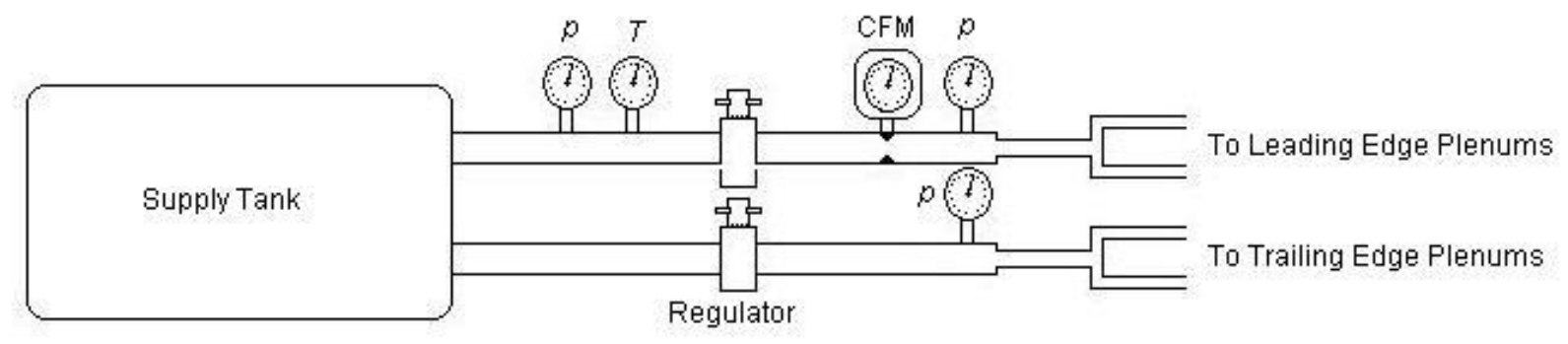

Figure 4.8: Supply Air System and Measurement Locations (Not drawn to scale or shape) 


\subsection{Facilities, Model, and Instrument Experimental Procedures}

Before testing could begin, proper calibration of each of the instruments was required.

\subsubsection{Facility/Environmental Study}

An environmental study for this experiment was performed by means of a wind tunnel frequency experiment (also documented by Lyons, 2008) to determine if any environmental variables or wind tunnel components such as breakers, fans, lights, motor vibrations, fan blade turbulence, etc. significantly contributed to the calculation of the forces. Another purpose of the study was to determine if any excessive deflection or vibration warranted a redesign to the test stand to maintain geometric stability.

Table 4.1 shows the average frequency and voltage magnitudes for the various environmental/wind tunnel components tested. Figure 4.9 shows the spectrum of the various wind tunnel component contributions. From these results, the only significant frequencies found in the frequency experiment were the $14-16 \mathrm{~Hz}$ natural frequency of the sting apparatus and the frequency generated by the motor (20 Hz at $1200 \mathrm{RPM})$. Since the amplitudes of oscillation were small compared to actual loadings of the airfoil (approximately $0.28 \mathrm{lbs}$ compared to $30 \mathrm{lbs}$ ), these frequencies were neglected as the data was sampled at $1000 \mathrm{~Hz}$ and averaged over a sampling time of 3 seconds. Therefore the natural frequencies and the forces generated from the various environmental and wind tunnel test conditions on the tunnel, stand, and sting were neglected.

Table 4.1: Average Frequency and Voltage Magnitudes for Various Wind Tunnel Components [Lyons, 2008]

\begin{tabular}{|c|c|c|}
\hline Testing Condition & $\mathrm{Hz}$ & Voltage Mag V \\
\hline \multirow{3}{*}{ Environment } & 22 & $3.00 \mathrm{e}-05$ \\
\cline { 2 - 3 } & 5149 & $3.92 \mathrm{e}-05$ \\
\cline { 2 - 3 } & 9444 & $2.54 \mathrm{e}-05$ \\
\hline Lights & 5614 & $4.48 \mathrm{e}-05$ \\
\hline Motor On at 595 RPM & 10 & $8.10 \mathrm{e}-05$ \\
\hline Motor On at 1204 RPM & 20 & $1.36 \mathrm{e}-04$ \\
\hline Motor On at 1204 RPM 50 fps & 14 & $1.80 \mathrm{e}-04$ \\
\hline \multirow{3}{*}{ Pressure Pump On } & 996 & $2.50 \mathrm{e}-05$ \\
\cline { 2 - 3 } & 3985 & $4.03 \mathrm{e}-05$ \\
\cline { 2 - 3 } & 6513 & $4.50 \mathrm{e}-05$ \\
\hline Blowing On & 847 & $2.33 \mathrm{e}-05$ \\
\hline
\end{tabular}




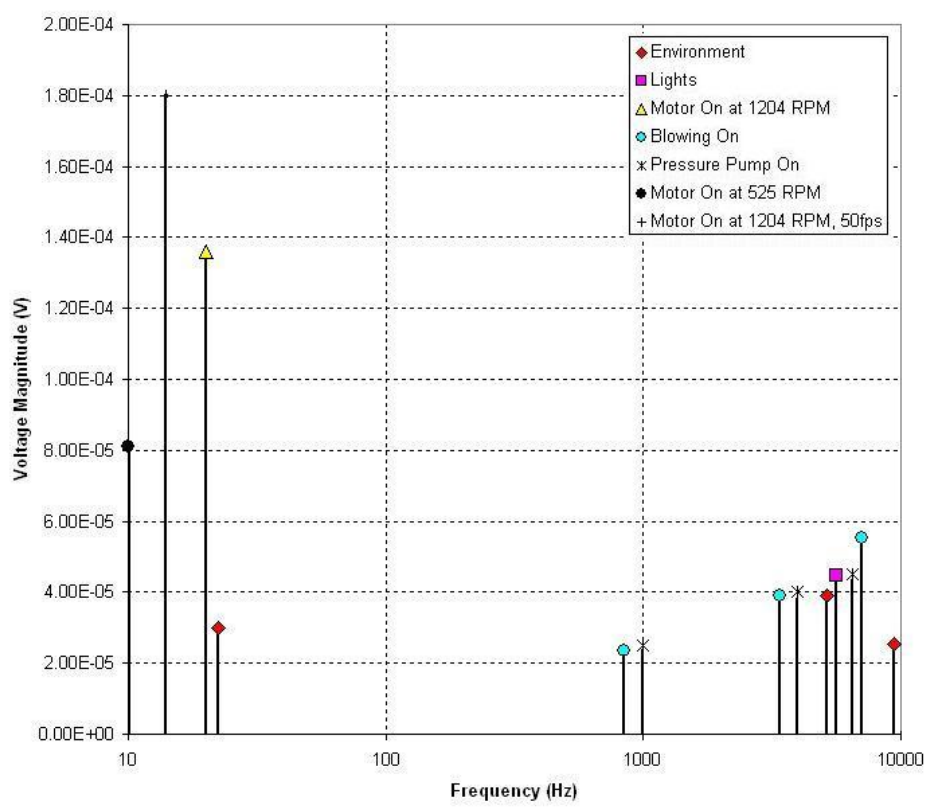

Figure 4.9: X-Axis Logarithmic Plot of the Average Frequency Components [Lyons, 2008]

A Finite Element Analysis was also performed on the test stand to simulate its deflection by the maximum forces shown in Figure 4.6. The manufacturer's recommended maximum force was applied to each load cell in the analysis. In addition to these loads, a force was applied in the vertical direction to simulate the weight of the model. The results of this study indicate that no significant deflection or force generation would occur from the maximum projected aerodynamic loadings. Therefore, these loadings would not provide any impact on the experimental readings including the geometry or the force calculation. 


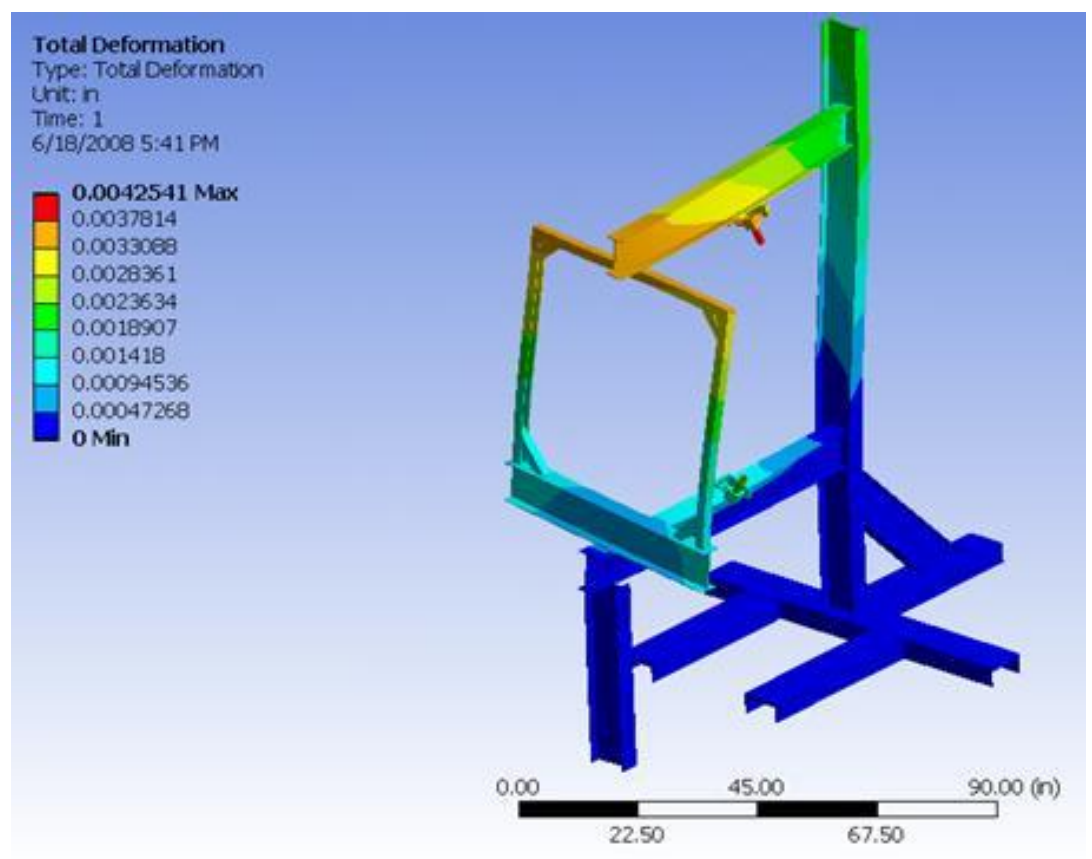

Figure 4.10: Exaggerated Deflection of the WVU Circulation Controlled model stand [Lyons, 2008]

To summarize this section, the deflection and vibration on the test stand generated from aerodynamic loading and from the natural environment including the test conditions were small enough to be neglected for this experiment.

\subsubsection{Load Cell Calibration}

The next step after proving experimentally that the testing environment and test stand were rigid (with respect to the sampled data set) was force calibration. The forces were measured by four S-beam type load cells and were calibrated with the model in place in the wind tunnel. Two load cells were attached at the top and bottom of the sting: one in the freestream (drag) direction, and one normal to the freestream (lift) direction. Forces were applied in the positive and negative direction in each load cell axis and recorded. These forces were applied to the load cells through the use of a pulley system and a calibrated weight set. Seven-point regression equations were then fit to each load cell's response to the various input forces. These equations were used to transform the voltages into forces. Because of the complexity of the system, gain matrices (such as Equation (4.1)) were used to calibrate the group of load cells and were treated as if they were a force balance as outlined in Barlow, Rae, and Pope, 1999 and in Appendix A. 


$$
\left\{\begin{array}{l}
F_{T L_{A}} \\
F_{T D_{A}} \\
F_{B L_{A}} \\
F_{B D_{A}}
\end{array}\right\}=\left[\begin{array}{llll}
K_{T L_{R} T L_{A}} & K_{T L_{R} T D_{A}} & K_{T L_{R} B L_{A}} & K_{T L_{R} B D_{A}} \\
K_{T D_{R} T L_{A}} & K_{T D_{R} T D_{A}} & K_{T D_{R} B L_{A}} & K_{T D_{R} B D_{A}} \\
K_{B L_{R} T L_{A}} & K_{B L_{R} T D_{A}} & K_{B L_{R} B L_{A}} & K_{B L_{R} B D_{A}} \\
K_{B D_{R} T L_{A}} & K_{B D_{R} T D_{A}} & K_{B D_{R} B L_{A}} & K_{B D_{R} B D_{A}}
\end{array}\right]^{-1}\left\{\begin{array}{l}
F_{T L_{R}} \\
F_{T D_{R}} \\
F_{B L_{R}} \\
F_{B D_{R}}
\end{array}\right\}
$$

The calibration testing scheme, definitions, methodology, and gain matrix calculations are presented in more detail in Appendix A as well as a comparison of various force calculation techniques.

\subsubsection{Angle-of-Attack Tare / Final Force Calculation}

Preliminary inspection of the model setup during one of the calibrations revealed that the forces applied to the model changed with variation of the angle of attack. This was not intended in the original design, but accounted for here. Thus, a small experiment was performed, recording the values of the forces at all test angles of attack in a lookup table. These force values were simply subtracted from the read value of the forces. Some unintentional shifting and movement of the model was also observed resulting in an inconsistent tare table for the angle of attack tare. To combat this inconsistency, the tare experiment was performed 4 times throughout the experiment and averaged. Figure 4.11 and Figure 4.12 show the lift and drag results of the four angle of attack tare experiments along with the average tare values.

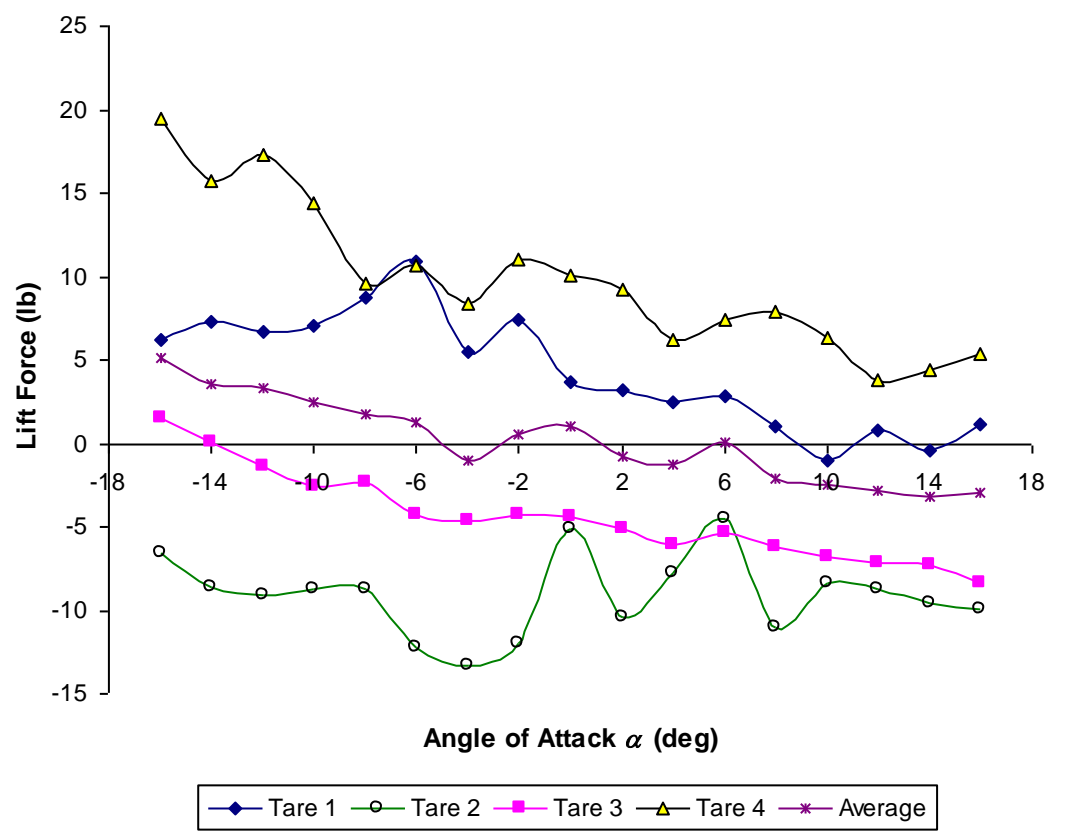

Figure 4.11: Angle of Attack Tare Values for Lift 


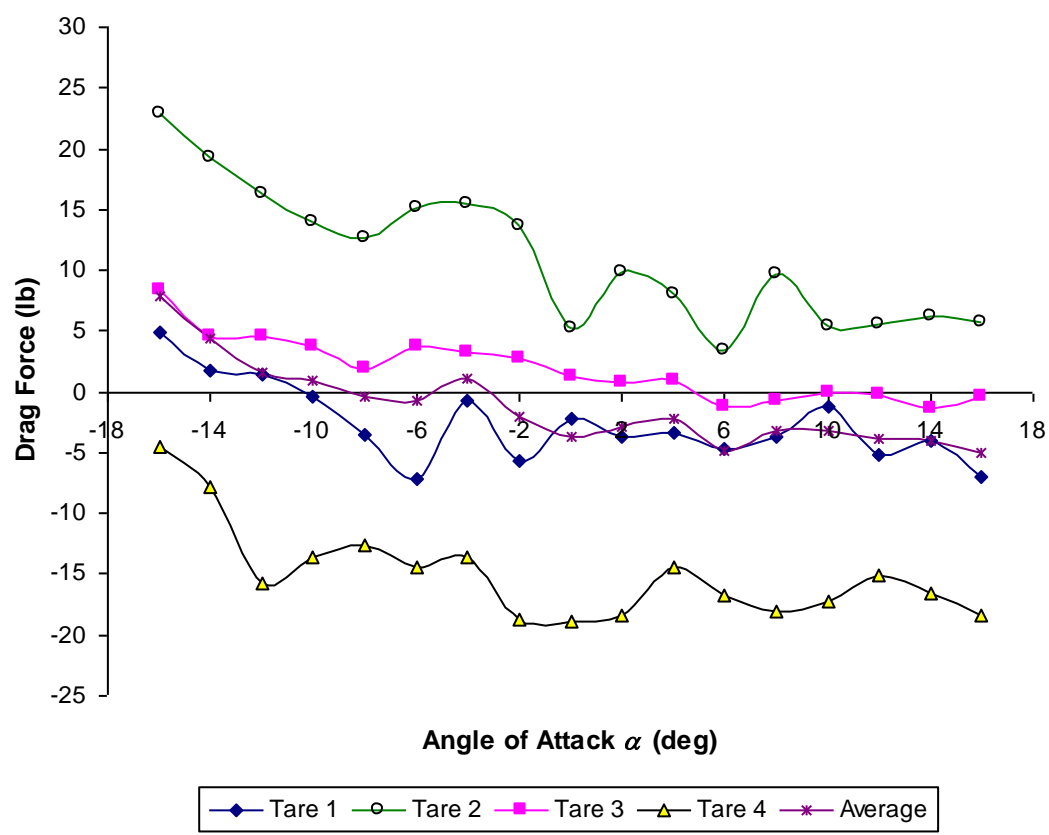

Figure 4.12: Angle of Attack Tare Values for Drag

Two methods of data reduction were investigated. The first method was to apply each individual angle of attack tare table until the next experiment was performed. For example, the first tare table was used to compensate for the loading caused by the change in angle of attack of the model in all the tests performed until the next tare table was created. The second method was to use the averaged tare table for all experimental runs.

In both methods, the forces from the top and bottom were summed to get the uncorrected lift and drag forces as shown in Equation (4.2) and Equation (4.3). The values for the tare forces at the appropriate angle of attack were then subtracted from the uncorrected forces to produce the corrected lift and drag forces as shown by Equation (4.4) and Equation (4.5). This concluded the calibration and tare methodology for the force calculation in the experiment. The forces were then input directly into the equations in Section 4.2.4 to provide useful data for comparison and analysis.

$$
\begin{gathered}
F_{L, u}=F_{T L}+F_{B L} \\
F_{D, u}=F_{T D}+F_{B D} \\
F_{L}(\alpha)=F_{L, u}(\alpha)-F_{L, \text { tare }}(\alpha) \\
F_{D}(\alpha)=F_{D, u}(\alpha)-F_{D, \text { tare }}(\alpha)
\end{gathered}
$$

\subsubsection{Lift and Drag Coefficient Calculation and Correction}

Once the measured voltages were calibrated and transformed into the force values, the lift and drag coefficients were calculated and corrections were applied. The lift and drag coefficients are normalized parameters 
useful for comparison of data from one experiment to another. These coefficients are calculated by Equations (4.6) and (4.7) from Anderson, 2001, respectively. The lift and drag forces were calculated by measuring the voltages on the load cells as described by the procedures outlined in Section 4.2.3. The density was calculated by using the atmospheric pressure, measured by an absolute pressure sensor, and the temperature, measured by a thermometer located along the tunnel wall in Equation (4.8) from Mills, 1995. These two parameters were measured at the beginning and end of every test run and averaged to provide a constant value over the full test range of angles of attack. The surface area was easily calculated by multiplying the chord of the model by the span of the model as shown in Equation (4.9).

$$
\begin{gathered}
c_{l}=\frac{2 L}{\rho V^{2} S} \\
c_{d}=\frac{2 D}{\rho V^{2} S} \\
\rho=\frac{p}{R T} \\
S=c \cdot b
\end{gathered}
$$

The velocity in the test section was calculated using Equation (4.10) from Anderson, 2001 and the weight per unit volume of water was found by multiplying the density of water by the local gravitational constant as shown in Equation (4.11). The density of water was found by Equation (4.12) from Jones, 1995 and the local gravity was found using the National Geodetic Survey Gravity Prediction Method from Fury, 2008 and Jekeli, 1994, at the latitude, longitude, and height above mean sea level of the test facility. The change in pressure was measured from subtracting the static pressure of the beginning of the test section from the wind tunnel reservoir (tunnel head) and the density was calculated using a variation of the ideal gas law, shown as Equation (4.8), from Bertin, 2002, where the absolute pressure is read from an absolute pressure sensor and the temperature is read from a spring scale thermometer needle gauge. The parameter $X$ was a near-unity Bernoulli correction for the change in areas and is described by Equation (4.13). The area ratio was determined from the cross sectional areas of the reservoir and test section which are $60 \mathrm{ft}^{2}$ and $10 \mathrm{ft}^{2}$, respectively, where the cross-sectional areas were determined by Equation (4.14) and (4.15) with the height and width measured by hand.

$$
\begin{gathered}
V=\sqrt{\frac{2 w \Delta h_{T}}{\rho X}} \\
w=\rho_{H_{2} O} \cdot g \\
\rho_{\mathrm{H}_{2} \mathrm{O}}=999.84847+0.06337563 T-8.523829 \times 10^{-3} T^{2}+6.94328 \times 10^{-5} T^{3}-3.821216 \times 10^{-7} T^{4} \\
X=1-\left(A_{\text {test }} / A_{\text {reservoir }}\right)^{2} \\
A_{\text {test }}=h_{\text {test }} w_{\text {test }} \\
A_{\text {res }}=h_{\text {res }} w_{\text {res }}
\end{gathered}
$$


The Reynold's number was then calculated by Equation (4.16), from Anderson, 2001, with the dynamic viscosity being described by Equation (4.17), from the Staff of NACA, 1953.

$$
\begin{gathered}
\operatorname{Re}=\frac{\rho V c}{\mu} \\
\mu=3.74 \times 10^{-7}\left(\frac{T}{518.6}\right)^{0.76}
\end{gathered}
$$

To account for tunnel wall interference effects, corrections to the various aerodynamic parameters were applied (Barlow, Rae, and Pope, 1999). Based on the availability of equipment, the method chosen for the correction of the lift and drag coefficients was the Allen and Vincenti method (Allen and Vincenti, 1944). This method was used by Abbot and Von Doenhofft in the data collection for his "Theory of Wing Sections" book (Abbot and Von Doenhofft, 1949). The first step in the correction was to find the base factor of the airfoil under investigation. The $10 \%$ thick ellipse base factor was not available in Allen and Vincenti, 1944. Therefore, a simple 5 point regression equation was fit to the elliptical airfoil base factor data provided Table 4.2. Note that the shaded value in the table was the generated value while the other values were the referenced values. Figure 4.13 shows the graph of some values from Table 4.2 and the regression equation used to generate the desired value for the $10 \%$ elliptical base factor. This equation is also described in Equation (4.18).

Table 4.2: Values for Base Factors $\Lambda$ from Allen and Vincenti, 1944

\begin{tabular}{|c|c|c|c|c|c|}
\hline $\mathrm{t} / \mathrm{c}$ & $\begin{array}{c}\text { Rankine } \\
\text { Oval }\end{array}$ & Ellipse & $\begin{array}{c}\text { Joukowski } \\
\text { section }\end{array}$ & $\begin{array}{c}\text { Conventional } \\
\text { NACA sections } \\
\text { 00XX }\end{array}$ & $\begin{array}{c}\text { NACA } \\
\text { Low-drag } \\
\text { 07-0XX }\end{array}$ \\
\hline & & & & & \\
\hline 0.06 & - & 0.127 & - & 0.111 & 0.125 \\
\hline 0.09 & 0.236 & 0.196 & 0.155 & 0.172 & 0.190 \\
\hline 0.10 & 0.264 & 0.222 & 0.174 & 0.195 & 0.218 \\
\hline 0.12 & 0.320 & 0.269 & 0.212 & 0.237 & 0.264 \\
\hline 0.15 & 0.403 & 0.345 & 0.273 & 0.305 & 0.342 \\
\hline 0.18 & 0.493 & 0.425 & 0.337 & 0.376 & 0.425 \\
\hline 0.21 & 0.580 & 0.508 & 0.404 & 0.450 & 0.512 \\
\hline 0.25 & 0.703 & 0.625 & 0.497 & 0.554 & 0.632 \\
\hline 0.30 & 0.864 & 0.780 & 0.626 & - & - \\
\hline 0.35 & 1.049 & 1.450 & 0.767 & - & - \\
\hline 0.50 & 1.690 & 1.500 & 1.258 & - & - \\
\hline 1.00 & 4.000 & 4.000 & - & - & - \\
\hline
\end{tabular}



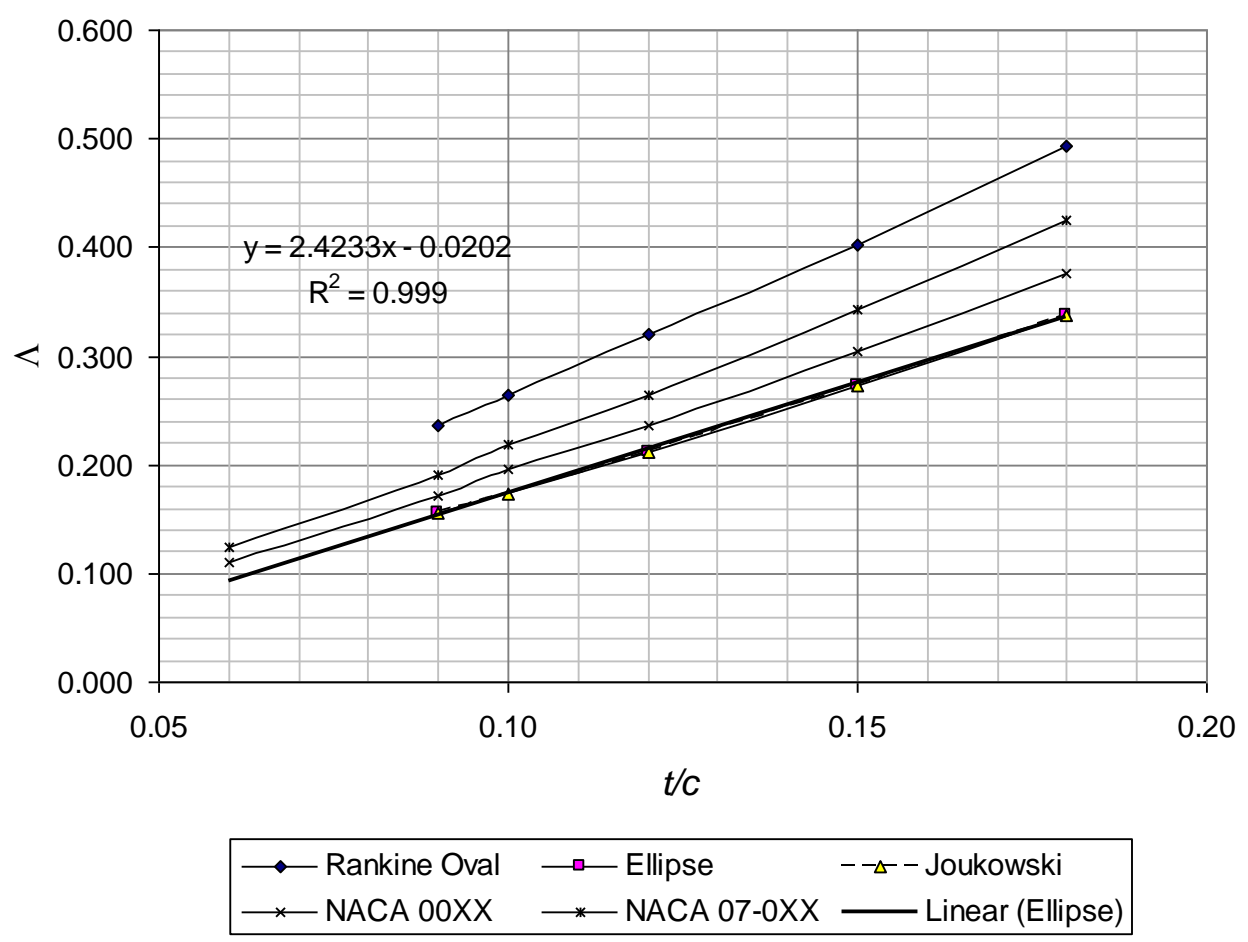

Figure 4.13: Base Factors from Various Airfoils (Generated from Allen and Vincenti, 1944)

Once the base factor was chosen, the chord-to-height ratio was determined and the parameters $\tau$ and $\sigma$ were calculated by Equation (4.19) and Equation (4.20), respectively. The uncorrected velocity, lift and drag coefficient, and angle of attack, denoted by the prime superscript, were then input, along with the base factor and the recently calculated parameters, $\tau$ and $\sigma$, into Equations (4.21)-(4.24) from Allen and Vincenti, 1949 to determine the corrected values. The velocity, lift coefficient, and drag coefficient were corrected using this approach.

$$
\begin{gathered}
\Lambda=2.4233\left(\frac{t}{c}\right)-0.0202 \\
\tau=\frac{c / h}{4} \\
\sigma=\frac{\pi^{2}}{48}\left(\frac{c}{h}\right)^{2} \\
V=V^{\prime}\left(1+\Lambda \sigma+\tau c_{d}{ }^{\prime}\right) \\
c_{l}=c_{l}{ }^{\prime}\left(1-\sigma-2 \Lambda \sigma-2 \tau c_{d}^{\prime}\right) \\
c_{d}=c_{d}{ }^{\prime}\left(1-3 \Lambda \sigma-2 \pi c_{d}{ }^{\prime}\right) \\
\alpha=\alpha^{\prime}+\frac{57.3 \sigma}{2 \pi}\left(c_{l}{ }^{\prime}+4 c_{m_{c / 4}}{ }^{\prime}\right)
\end{gathered}
$$


This concludes the calculation and applied corrections for two of the main parameters under investigation in this experiment, the lift and drag coefficients. The last coefficient, the blowing coefficient, is discussed in the next section.

\subsubsection{Jet Velocity and Blowing Coefficient Determination}

The last main parameter to be determined in this investigation was the blowing coefficient. This parameter non-dimensionalized the circulation control applied to the airfoil, allowing useful comparison between the current and previous experiments. To properly determine this coefficient, a detailed analysis was performed. The assumptions that the model maintained the same geometry as the design geometry or that the blowing velocities were uniform across the model were invalid and were validated experimentally.

The first step in determining the blowing coefficient was to model the geometry of the blowing slots. Specifically important is the area of the jets, determined by integrating the height of the slots along the span of the model. Measurements of slot height were taken at one or less inch intervals along the 30.94 inch span. The normalized slot height of the leading and trialing edges of the model are shown in Figure 4.14.

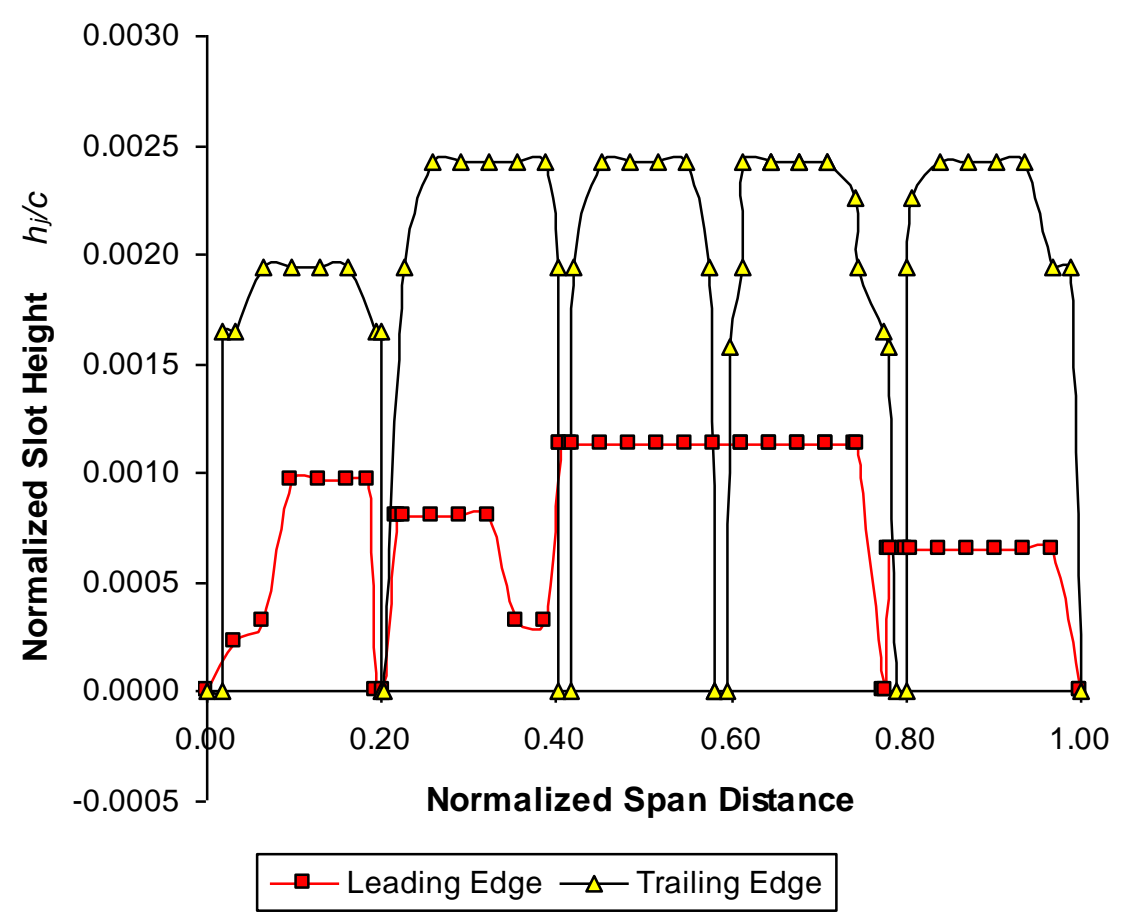

Figure 4.14: Normalized Slot Height for CCHB Model 
Next, measurements of differential pressure were made with a pitot static probe at one or less inch intervals. Because of the difficulty of measuring the complex flow of the blowing slots (example: entire flow stream from blowing slots is on the same order of magnitude as the probe), the magnitudes of the local velocities calculated from the simple pressure differential, as shown by Eq. (4.25) from Anderson, 2001, as well as the angles of the blowing velocities are arguably invalid. However, the normalized distribution of the velocities is useful. By comparing the normalized velocities of the two different supply pressures, the distribution profile can be validated.

$$
V_{j, 1}=\sqrt{\frac{2 \Delta P}{\rho_{\text {atm }}}}
$$

The normalized velocities profiles of the two different supply pressures are shown in Figure 4.15 and Figure 4.16. Figure 4.17 shows the design maximum jet exit velocity angle (black arrow) and the measured maximum jet exit velocity angle variation (gray shaded area) along the span of the model.

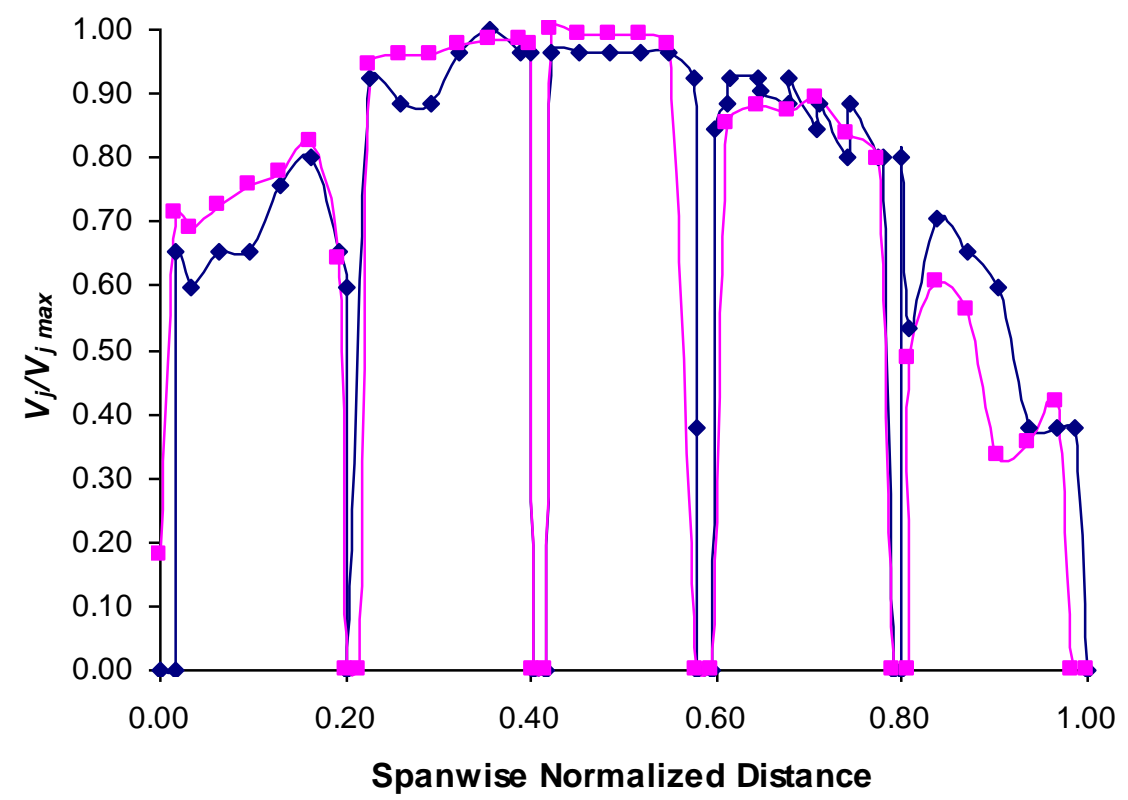

$\longrightarrow \bullet$ TE $10 \mathrm{psi} \leadsto-\mathrm{TE} 30 \mathrm{psi}$

Figure 4.15: Trailing Edge Normalized Velocity Profile 


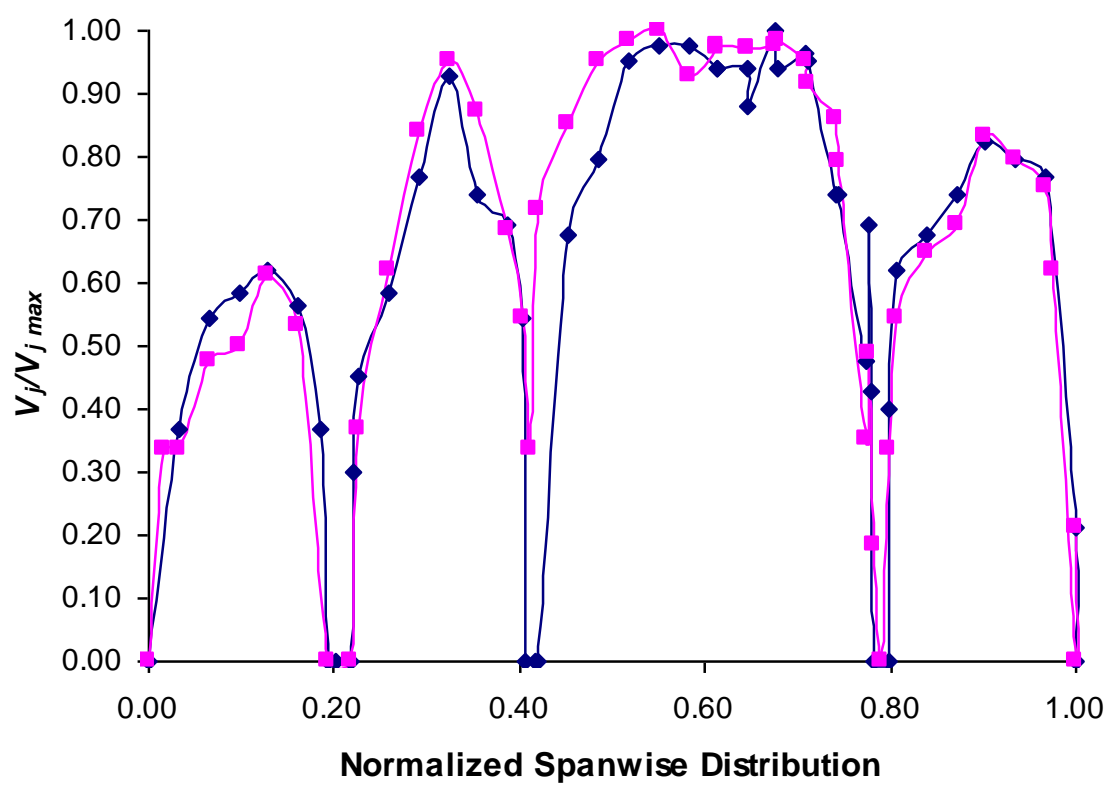

$\multimap-$ LE $10 \mathrm{psi} \rightarrow-\mathrm{LE} 20 \mathrm{psi}$

Figure 4.16: Leading Edge Normalized Velocity Profile

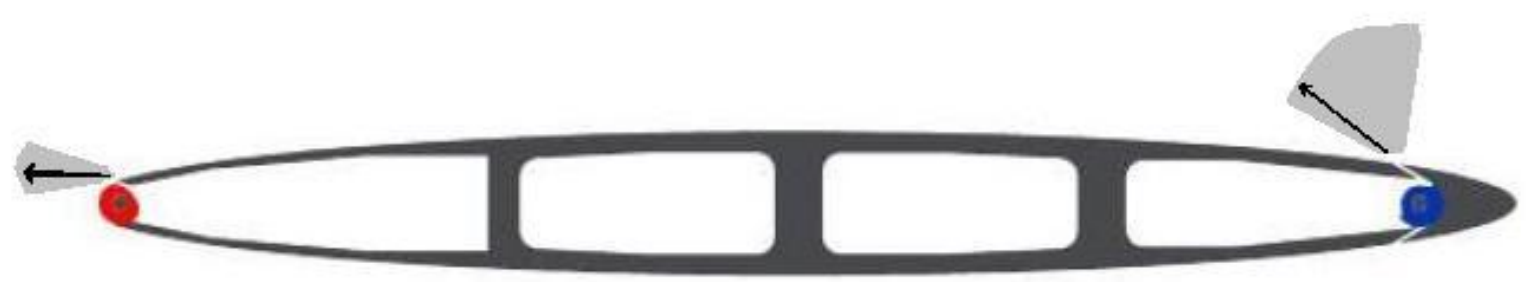

Figure 4.17: Blowing Slot Jet Maximum Velocity Direction 
The velocity profiles were then averaged by integration as shown in Eq. (4.26) and the total area found by Eq. (4.27). Unless otherwise noted, all integrations are performed by using the trapezoidal rule approximation.

$$
\begin{gathered}
\overline{V_{j, 1}}=\frac{\int V_{j, 1} d b}{b} \\
A_{j}=\int h_{j} d b
\end{gathered}
$$

Once the profiles were obtained, the magnitudes of the velocities and the corresponding mass flow rates of the leading and trailing edge blowing systems at various supply pressures were desired. The mass flow of the trailing edge was calculated by measuring the volumetric flow rate using a flow meter and by multiplying the results by the atmospheric density as shown in Eq. (4.28). Eq. (4.29) shows a relationship between the leading and trailing edge mass flows. Assuming that the density of the air in the supply lines for the trailing edge and leading edge are equal, this equation can be rearranged as shown in Eq. (4.30) to find the mass flow of the leading edge with the other parameters measured from the velocity distribution and trailing edge volumetric flow measurement. The average velocity magnitudes were then calculated using Eq. (4.31) for each test condition. The supply air temperature was read with an analog thermocouple and the atmospheric pressure was measured with a wall mounted analog barometer. These measurements were performed at near the same conditions the manufacturer used to calibrate the flow meter. The blowing coefficient was then calculated using Equation (4.32) from Alexander and Anders, 2005.

$$
\begin{gathered}
\dot{m}_{T E}=g_{c} * \rho_{p} * C F M / 60 \\
\frac{\dot{m}_{L E}}{\dot{m}_{T E}}=\frac{\rho_{L E} A_{L E} V_{j_{L E}}}{\rho_{T E} A_{T E} V_{j T E}} \\
\dot{m}_{L E}=\frac{A_{L E} \overline{V_{j, 1}}}{A_{T E} \overline{V_{j, 1}}} \dot{m}_{T E} \\
\overline{V_{j}}=\frac{\dot{m} / g_{c}}{\rho_{d} A_{j}} \\
c_{\mu}=\frac{\dot{m} V_{j}}{1 / 2 \rho V^{2} S}
\end{gathered}
$$

The results of the mass flow analysis are shown in Table 4.3 and plotted in Figure 4.18. The area and velocity ratios used in these calculations are shown in Table 4.4 . 
Table 4.3: Mass Flow and Jet Velocity Results

\begin{tabular}{|c|c|c|c|c|c|c|c|}
\hline Location & \multicolumn{7}{|c|}{ Trailing Edge } \\
\hline Descript. & Pressure & Volumetric Flow Rate & Mass Flow Rate & Jet Velocity & Momentum & Blowing & Blowing \\
\hline Units & psi & SCFM & $\mathrm{lb}_{\mathrm{m}} / \mathrm{sec}$ & $\mathrm{fps}$ & $\mathrm{lb}_{\mathrm{m}}-\mathrm{ft} / \mathrm{sec}^{2}$ & $c_{\mu} @ 80 \mathrm{fps}$ & $c_{\mu} @ 120 \mathrm{fps}$ \\
\hline \multirow{4}{*}{ Data } & 10 & 77 & 0.092 & 96.233 & 8.856 & 0.00340 & 0.00151 \\
\hline & 20 & 122 & 0.146 & 152.473 & 22.233 & 0.00853 & 0.00379 \\
\hline & 30 & 155 & 0.185 & 193.716 & 35.888 & 0.01377 & 0.00612 \\
\hline & 40 & 180 & 0.215 & 224.961 & 48.398 & 0.01857 & 0.00825 \\
\hline Location & \multicolumn{7}{|c|}{ Leading Edge } \\
\hline Descript. & Pressure & Volumetric Flow Rate & Mass Flow Rate & Jet Velocity & Momentum & Blowing & Blowing \\
\hline Units & $p s i$ & SCFM & $\mathrm{lb} / \mathrm{sec}$ & fps & $\mathrm{lb}_{\mathrm{m}}-\mathrm{ft} / \mathrm{sec}^{2}$ & $c_{\mu} @ 80 \mathrm{fps}$ & $c_{\mu} @ 120 \mathrm{fps}$ \\
\hline \multirow{4}{*}{ Data } & 10 & 25.959 & 0.058 & 153.843 & 8.908 & 0.00342 & 0.00152 \\
\hline & 20 & 43.175 & 0.096 & 255.867 & 24.641 & 0.00946 & 0.00420 \\
\hline & 30 & 57.451 & 0.128 & 340.471 & 43.631 & 0.01674 & 0.00744 \\
\hline & 40 & 69.735 & 0.156 & 413.270 & 64.283 & 0.02467 & 0.01096 \\
\hline
\end{tabular}

Table 4.4: Area and Velocity Ratios Used in Mass Flow Calculations

\begin{tabular}{|c|c|c|}
\hline Area Ratio & $\mathrm{A}_{\mathrm{jLE}} / \mathrm{A}_{\mathrm{jTE}}$ & 0.3936 \\
\hline \multirow{3}{*}{$\begin{array}{c}\text { Velocity } \\
\text { Ratio }\end{array}$} & $\mathrm{V}_{\mathrm{jLE}} / \mathrm{V}_{\mathrm{jTE}}$ at $10 \mathrm{psi}$ & 1.5987 \\
\cline { 2 - 3 } & $\mathrm{V}_{\mathrm{jLE}} / \mathrm{V}_{\mathrm{jTE}}$ at $20 \mathrm{psi}$ & 1.6781 \\
\cline { 2 - 3 } & $\mathrm{V}_{\mathrm{jLE}} / \mathrm{V}_{\mathrm{jTE}}$ at $30 \mathrm{psi}$ & 1.7576 \\
\cline { 2 - 3 } & $\mathrm{V}_{\mathrm{jLE}} / \mathrm{V}_{\mathrm{jTE}}$ at $40 \mathrm{psi}$ & 1.8371 \\
\hline
\end{tabular}

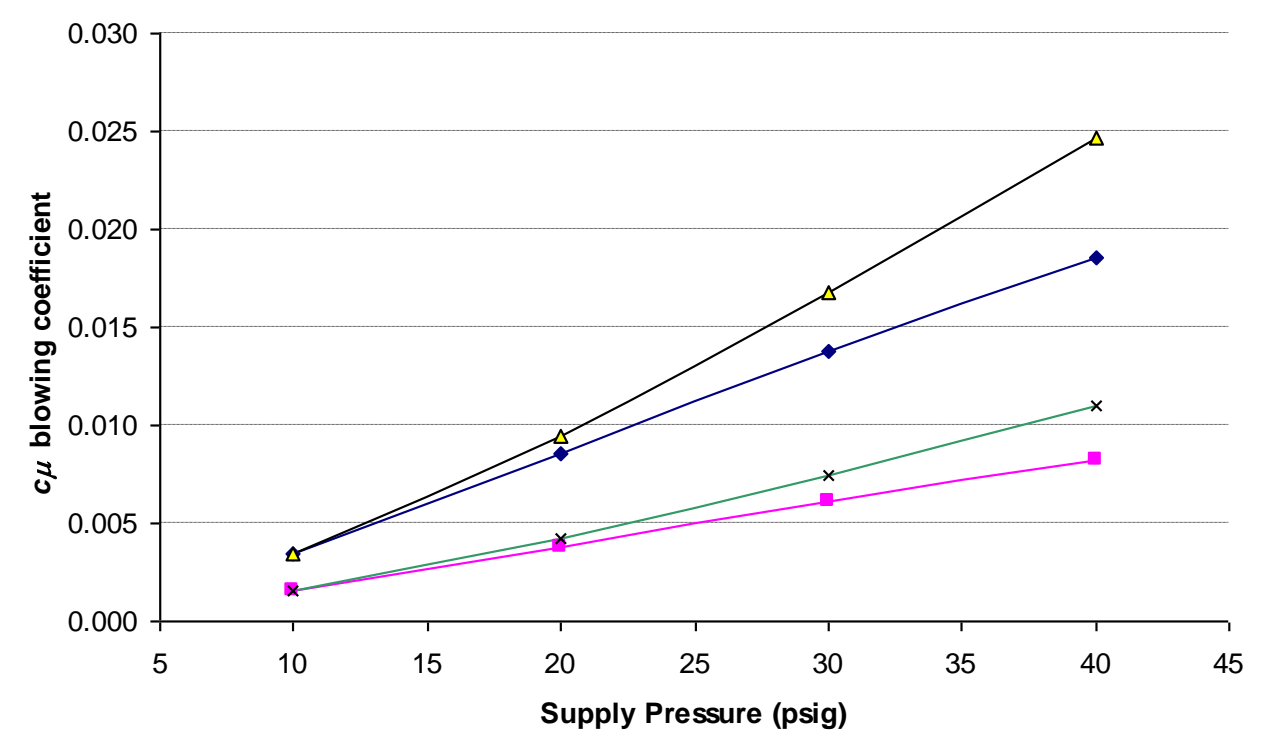

$\rightarrow-80$ fps TE Blowing --120 fps TE Blowing $\triangle-80$ fps LE Blowing $\rightarrow-120$ fps LE Blowing

Figure 4.18: Blowing Coefficient vs. Regulated Supply Pressure 


\subsection{Wind Tunnel Testing Schedule}

Once the experiment was setup and the proper calibrations and parameter corrections were applied as discussed previously, the actual testing of the airfoil could begin. The lift and drag coefficients, were selected to be tested independently of the leading and trailing edge blowing coefficients and the angle of attack. In terms of measurement, the lift and drag forces were measured independently of the leading and trailing edge blowing supply pressure and angle of attack. The blowing pressures of 0,10 , and $20 \mathrm{psi}$ for the leading edge blowing slots $\left(c_{\mu}=0\right.$, 0.0015 , and 0.0042$)$ and 0,10 , and 30 psi for the trailing edge blowing slots $\left(c_{\mu}=0,0.0015\right.$, and 0.0061$)$ were selected based on the storage capacity and time response of the air pressure supply system. The angle of attack test range was selected to be from 154 to 196 degrees, with 2 degree increments.

The lift and drag coefficients were also tested for Mach number independence. Again, from a measurement perspective, the lift and drag forces were measured independently of the freestream velocity at two test points, 80 fps and 120 fps. These test points lie within the recommended Mach number range of 0.05 to 0.2 where flow similarity and therefore lift and drag coefficient similarity should exist. The Mach number independence check is similar to a Reynold's number independence, but is not dependent on the viscosity. In this Mach number range, the lift and drag coefficients should not change as the Mach number changes.

All 9 conditions at 2 freestream velocities were tested 3 times and averaged for a total of 54 independent runs with 486 test points and 1,458,000 samples.

\subsection{Uncertainty analysis}

An uncertainty analysis was performed according to Beckwith's procedure (Beckwith, Marangoni, and Lienhard, 1995). This analysis showed the error in the results due to the uncertainty of the various measurement devices and parameters. These uncertainties are simply a vector sum of the partial derivatives of each contributing sub-parameter multiplied by its error as shown in Equation (4.33). The final equations for all the uncertainty percentages for the results are shown in Equations (4.34) through (4.44). The uncertainties are divided by the calculated value to show percentage of error and allow for easy manipulation. It is noted that: if an uncertainty parameter is not defined by another equation, it is a measured value or a manufacturer specification. 


$$
\begin{aligned}
& u_{y}=\left[\left(\frac{\partial y}{\partial x_{1}} u_{1}\right)^{2}+\left(\frac{\partial y}{\partial x_{2}} u_{2}\right)^{2}+\cdots+\left(\frac{\partial y}{\partial x_{n}} u_{n}\right)^{2}\right]^{1 / 2} \\
& \frac{W_{c_{l}}}{c_{l}}=\left[\left(\frac{W_{L}}{L}\right)^{2}+\left(\frac{W_{\rho}}{\rho}\right)^{2}+\left(\frac{2 W_{V}}{V}\right)^{2}+\left(\frac{W_{S}}{S}\right)^{2}\right]^{1 / 2} \\
& \frac{W_{c_{d}}}{c_{d}}=\left[\left(\frac{W_{D}}{D}\right)^{2}+\left(\frac{W_{\rho}}{\rho}\right)^{2}+\left(\frac{2 W_{V}}{V}\right)^{2}+\left(\frac{W_{S}}{S}\right)^{2}\right]^{1 / 2} \\
& \frac{W_{L}}{L}=\left[\left(\frac{W_{L}(\alpha)}{L}\right)^{2}+\left(\frac{W_{L_{\text {Calib }}}}{L}\right)^{2}\right]^{1 / 2} \\
& \frac{W_{\rho}}{\rho}=\left[\left(\frac{W_{p}}{p}\right)^{2}+\left(\frac{W_{T}}{T}\right)^{2}\right]^{1 / 2} \\
& \frac{W_{S}}{S}=\left[\left(\frac{W_{c}}{c}\right)^{2}+\left(\frac{W_{b}}{b}\right)^{2}\right]^{1 / 2} \\
& \frac{W_{V}}{V}=\left[\left(\frac{W_{w}}{2 w}\right)^{2}+\left(\frac{W_{\Delta h_{T}}}{2 \Delta h_{T}}\right)^{2}+\left(\frac{W_{\rho}}{2 \rho}\right)^{2}+\left(\frac{W_{X}}{2 X}\right)^{2}\right]^{1 / 2} \\
& \frac{W_{\mathrm{Re}}}{\operatorname{Re}}=\left[\left(\frac{W_{\rho}}{\rho}\right)^{2}+\left(\frac{W_{V}}{V}\right)^{2}+\left(\frac{W_{c}}{c}\right)^{2}+\left(\frac{W_{\mu}}{\mu}\right)^{2}\right]^{1 / 2} \\
& \frac{W_{w}}{w}=\left[\left(\frac{W_{\rho_{H_{2} O}}}{\rho_{H_{2} O}}\right)^{2}+\left(\frac{W_{g}}{g}\right)^{2}\right]^{1 / 2} \\
& W_{X}=\left[\left(2 W_{h_{\text {test }}} \frac{h_{\text {test }} w_{\text {test }}{ }^{2}}{h_{\text {res }}{ }^{2} w_{\text {res }}{ }^{2}}\right)^{2}+\left(2 W_{w_{\text {test }}} \frac{h_{\text {test }}{ }^{2} w_{\text {test }}}{h_{\text {res }}{ }^{2} w_{\text {res }}{ }^{2}}\right)^{2}+\left(2 W_{h_{\text {tres }}} \frac{h_{\text {test }}{ }^{2} w_{\text {test }}{ }^{2}}{h_{\text {res }}{ }^{3} w_{\text {res }}{ }^{2}}\right)^{2}+\left(2 W_{w_{\text {res }}} \frac{h_{\text {test }}{ }^{2} w_{\text {test }}{ }^{2}}{h_{\text {res }}{ }^{2} w_{\text {res }}{ }^{3}}\right)^{2}\right]^{1 / 2} \\
& W_{\rho_{H_{2} \mathrm{O}}}=0.06337563-2\left(8.523829 \times 10^{-3}\right) T+3\left(6.94328 \times 10^{-5}\right) T^{2}-4\left(3.821216 \times 10^{-7}\right) T^{3} \\
& \frac{W_{\mu}}{\mu}=\left[\left(\frac{W_{T}}{T}\right)^{2}\right]^{1 / 2} \\
& \frac{W_{\dot{m}}}{\dot{m}}=\left[\left(\frac{W_{g_{c}}}{g_{c}}\right)^{2}+\left(\frac{W_{\rho_{p}}}{\rho_{p}}\right)^{2}+\left(\frac{W_{C F M}}{C F M} / 60\right)^{2}\right]^{1 / 2} \\
& \frac{W_{\overline{V_{j}}}}{\overline{V_{j}}}=\left[\left(\frac{W_{g_{c}}}{g_{c}}\right)^{2}+\left(\frac{W_{\dot{m}}}{\dot{m}}\right)^{2}+\left(\frac{W_{\rho_{d}}}{\rho_{d}}\right)^{2}+\left(\frac{W_{A_{j}}}{A_{j}}\right)^{2}\right]^{1 / 2}
\end{aligned}
$$


An uncertainty calculation from the 80 fps freestream velocity case with no blowing is shown in Table 4.5 through Table 4.7. Figure 4.19 and Figure 4.20 show the error bar plots for the same case based on the uncertainties calculated. Since over 54 runs were performed, and the uncertainties from the various cases are highly similar in magnitude.only this case is shown.

It is concluded that uncertainties due to the final force calculation (as a function of angle of attack), the preliminary force calculation, mass flow of the jet, and the velocity of the jet are highly significant. The other uncertainty parameters provide only small contributions to the overall uncertainty of the experiment.

Table 4.5: Lift and Drag Coefficient Uncertainties (Sample: Tunnel Velocity = 80 fps, No Blowing)

\begin{tabular}{|c|c|c|c|c|}
\hline$\alpha(\mathrm{deg})$ & $W_{c_{l_{u}}}$ & $W_{c_{l}}$ & $W_{c_{d_{u}}}$ & $W_{c_{d}}$ \\
\hline-16 & 0.708 & 0.746 & 0.708 & 0.753 \\
\hline-14 & 0.612 & 0.634 & 0.611 & 0.639 \\
\hline-12 & 0.719 & 0.778 & 0.719 & 0.786 \\
\hline-10 & 0.621 & 0.663 & 0.619 & 0.668 \\
\hline-8 & 0.416 & 0.383 & 0.411 & 0.383 \\
\hline-6 & 0.516 & 0.558 & 0.515 & 0.562 \\
\hline-4 & 0.496 & 0.529 & 0.496 & 0.534 \\
\hline-2 & 0.550 & 0.585 & 0.550 & 0.591 \\
\hline 0 & 0.471 & 0.454 & 0.471 & 0.459 \\
\hline 2 & 0.527 & 0.486 & 0.527 & 0.492 \\
\hline 4 & 0.399 & 0.354 & 0.399 & 0.358 \\
\hline 6 & 0.397 & 0.350 & 0.395 & 0.353 \\
\hline 8 & 0.529 & 0.454 & 0.529 & 0.459 \\
\hline 10 & 0.463 & 0.406 & 0.462 & 0.410 \\
\hline 12 & 0.338 & 0.305 & 0.337 & 0.308 \\
\hline 14 & 0.385 & 0.345 & 0.384 & 0.349 \\
\hline
\end{tabular}


Table 4.6: Lift and Drag Force Uncertainties (Tunnel Velocity $=80 \mathrm{fps}$, No Blowing)

\begin{tabular}{|c|c|c|c|c|}
\hline$\alpha(\operatorname{deg})$ & $W_{L}(+)$ & $W_{L}(-)$ & $W_{D}(+)$ & $W_{D}(-)$ \\
\hline-16 & 14.264 & 11.728 & 15.047 & 12.478 \\
\hline-14 & 12.114 & 12.220 & 14.821 & 12.225 \\
\hline-12 & 13.968 & 12.495 & 14.626 & 17.446 \\
\hline-10 & 11.889 & 11.285 & 13.057 & 14.573 \\
\hline-8 & 7.845 & 10.604 & 13.090 & 12.247 \\
\hline-6 & 9.685 & 13.536 & 15.865 & 13.777 \\
\hline-4 & 9.409 & 12.334 & 14.456 & 14.763 \\
\hline-2 & 10.453 & 12.492 & 15.632 & 16.741 \\
\hline 0 & 8.981 & 6.149 & 8.887 & 15.183 \\
\hline 2 & 9.962 & 9.611 & 12.667 & 15.526 \\
\hline 4 & 7.460 & 6.474 & 10.232 & 12.227 \\
\hline 6 & 7.414 & 5.515 & 8.270 & 11.998 \\
\hline 8 & 10.015 & 9.036 & 12.948 & 14.951 \\
\hline 10 & 8.862 & 5.899 & 8.757 & 13.960 \\
\hline 12 & 6.613 & 5.950 & 9.300 & 11.364 \\
\hline 14 & 7.610 & 6.348 & 10.161 & 12.663 \\
\hline
\end{tabular}

Table 4.7: Uncertainties of Various Parameters (Tunnel Velocity $=80$ fps, No Blowing)

\begin{tabular}{|c|c|c|c|c|c|c|c|c|c|c|}
\hline$\alpha(\mathrm{deg})$ & $W_{\alpha}(\mathrm{deg})$ & $W_{T}$ & $W_{p}$ & $W_{\Delta h_{T}}$ & $W_{\mu}$ & $W_{\rho}$ & $W_{V_{u}}$ & $W_{V}$ & $W_{\mathrm{Re}_{u}}$ & $W_{\mathrm{Re}}$ \\
\hline $\mathrm{deg}$ & $\mathrm{deg}$ & ${ }^{\circ} \mathrm{R}$ & $\mathrm{psia}$ & $\mathrm{in}_{2} \mathrm{O}$ & $\mathrm{lb}_{\mathrm{m}} /(\mathrm{ft}-\mathrm{sec})$ & \multicolumn{1}{|c|}{ slugs $/ \mathrm{ft}^{3}$} & $\mathrm{fps}$ & $\mathrm{fps}$ & - & - \\
\hline-16 & 0.477 & 1 & 0.005 & $2.92 \mathrm{E}-04$ & $7.14 \mathrm{E}-10$ & $4.09 \mathrm{E}-06$ & 0.4096 & 0.3958 & 2905 & 2807 \\
\hline-14 & 0.477 & 1 & 0.005 & $2.92 \mathrm{E}-04$ & $7.14 \mathrm{E}-10$ & $4.09 \mathrm{E}-06$ & 0.4067 & 0.3965 & 2883 & 2811 \\
\hline-12 & 0.477 & 1 & 0.005 & $2.92 \mathrm{E}-04$ & $7.14 \mathrm{E}-10$ & $4.09 \mathrm{E}-06$ & 0.4031 & 0.3837 & 2856 & 2719 \\
\hline-10 & 0.477 & 1 & 0.005 & $2.92 \mathrm{E}-04$ & $7.14 \mathrm{E}-10$ & $4.09 \mathrm{E}-06$ & 0.4010 & 0.3845 & 2841 & 2724 \\
\hline-8 & 0.477 & 1 & 0.005 & $2.92 \mathrm{E}-04$ & $7.14 \mathrm{E}-10$ & $4.09 \mathrm{E}-06$ & 0.3999 & 0.4129 & 2832 & 2924 \\
\hline-6 & 0.477 & 1 & 0.005 & $2.92 \mathrm{E}-04$ & $7.14 \mathrm{E}-10$ & $4.09 \mathrm{E}-06$ & 0.3975 & 0.3787 & 2814 & 2681 \\
\hline-4 & 0.477 & 1 & 0.005 & $2.92 \mathrm{E}-04$ & $7.14 \mathrm{E}-10$ & $4.09 \mathrm{E}-06$ & 0.3991 & 0.3829 & 2826 & 2712 \\
\hline-2 & 0.477 & 1 & 0.005 & $2.92 \mathrm{E}-04$ & $7.14 \mathrm{E}-10$ & $4.09 \mathrm{E}-06$ & 0.3993 & 0.3838 & 2827 & 2718 \\
\hline 0 & 0.477 & 1 & 0.005 & $2.92 \mathrm{E}-04$ & $7.14 \mathrm{E}-10$ & $4.09 \mathrm{E}-06$ & 0.3999 & 0.4042 & 2832 & 2863 \\
\hline 2 & 0.477 & 1 & 0.005 & $2.92 \mathrm{E}-04$ & $7.14 \mathrm{E}-10$ & $4.09 \mathrm{E}-06$ & 0.3984 & 0.4110 & 2821 & 2910 \\
\hline 4 & 0.477 & 1 & 0.005 & $2.92 \mathrm{E}-04$ & $7.14 \mathrm{E}-10$ & $4.09 \mathrm{E}-06$ & 0.3969 & 0.4167 & 2809 & 2950 \\
\hline 6 & 0.477 & 1 & 0.005 & $2.92 \mathrm{E}-04$ & $7.14 \mathrm{E}-10$ & $4.09 \mathrm{E}-06$ & 0.3975 & 0.4179 & 2814 & 2959 \\
\hline 8 & 0.477 & 1 & 0.005 & $2.92 \mathrm{E}-04$ & $7.14 \mathrm{E}-10$ & $4.09 \mathrm{E}-06$ & 0.3989 & 0.4245 & 2825 & 3006 \\
\hline 10 & 0.477 & 1 & 0.005 & $2.92 \mathrm{E}-04$ & $7.14 \mathrm{E}-10$ & $4.09 \mathrm{E}-06$ & 0.4011 & 0.4230 & 2841 & 2996 \\
\hline 12 & 0.477 & 1 & 0.005 & $2.92 \mathrm{E}-04$ & $7.14 \mathrm{E}-10$ & $4.09 \mathrm{E}-06$ & 0.4049 & 0.4220 & 2869 & 2991 \\
\hline 14 & 0.477 & 1 & 0.005 & $2.92 \mathrm{E}-04$ & $7.14 \mathrm{E}-10$ & $4.09 \mathrm{E}-06$ & 0.4066 & 0.4249 & 2882 & 3012 \\
\hline
\end{tabular}




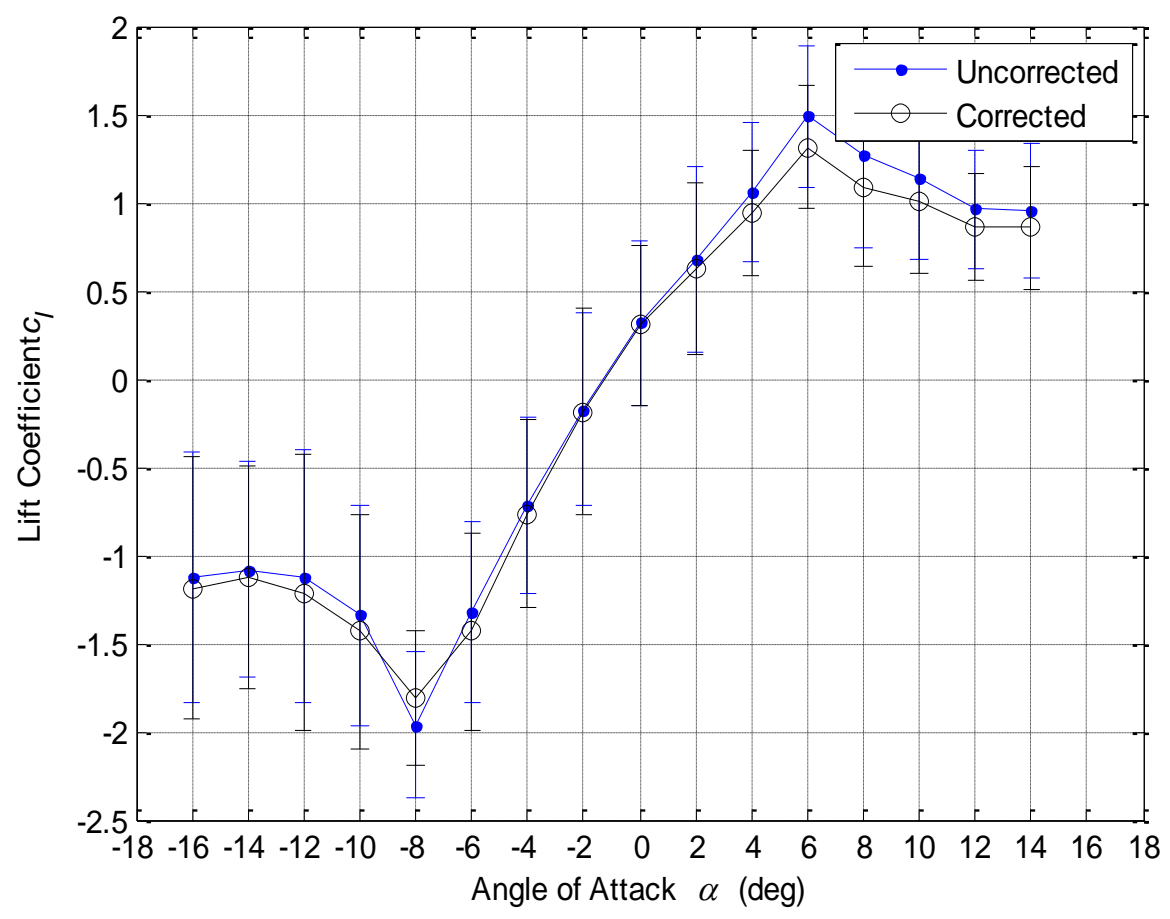

Figure 4.19: Lift Coefficient Error Bar Plot (Tunnel Velocity $=80$ fps, no blowing)

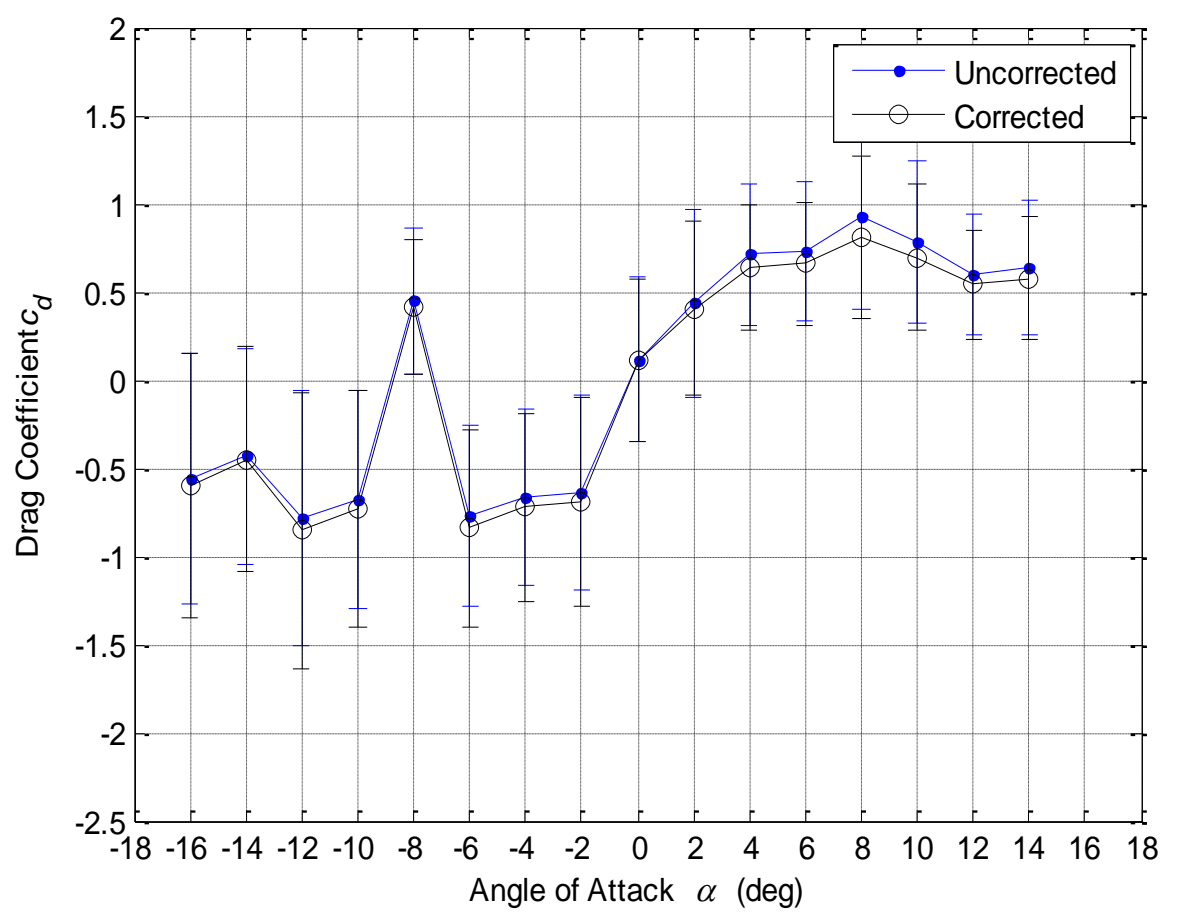

Figure 4.20: Drag Coefficient Error Bar Plot (Tunnel Velocity $=80$ fps, no blowing) 


\section{Chapter 5: Summarized Results}

This section summarizes the results of this investigation which includes the comparison between this and prior experiments' findings for the airfoil's lift and drag coefficients under no-blowing conditions as well as lift coefficient plots for the blown conditions that summarize the findings of this experiment. A table is also provided to easily compare the different blowing configurations' results. Higher detail results are presented in a graphical format in Appendix B.

\subsection{Results for Lift and Drag Coefficients under the No-Blowing Condition}

Figure 5.1 and Figure 5.2 show the results from the current experiment compared to the previous experimental studies for the lift and drag coefficients in reverse flow under the no blowing condition, with the exception that Alexander and Anders's (2005) results were for forward flow. The shape of the lift coefficient was similar to previous experiments. However, the magnitude was much higher, with the negative angles of attack showing a greater discrepancy, falling outside the range of error. Based on these results, Mach number independence was assumed.

The shape and the magnitude of the drag coefficient were dissimilar to previous experimental results but fell within the range of error for the majority of the test points. One possible reason for such dissimilarity is the measurement method for this parameter. As stated earlier, and by Englar and Williams, 1975, drag results using this method are highly inaccurate. Another possible reason for the discrepancies was the unintentional shifting of the test stand and sting apparatus. 


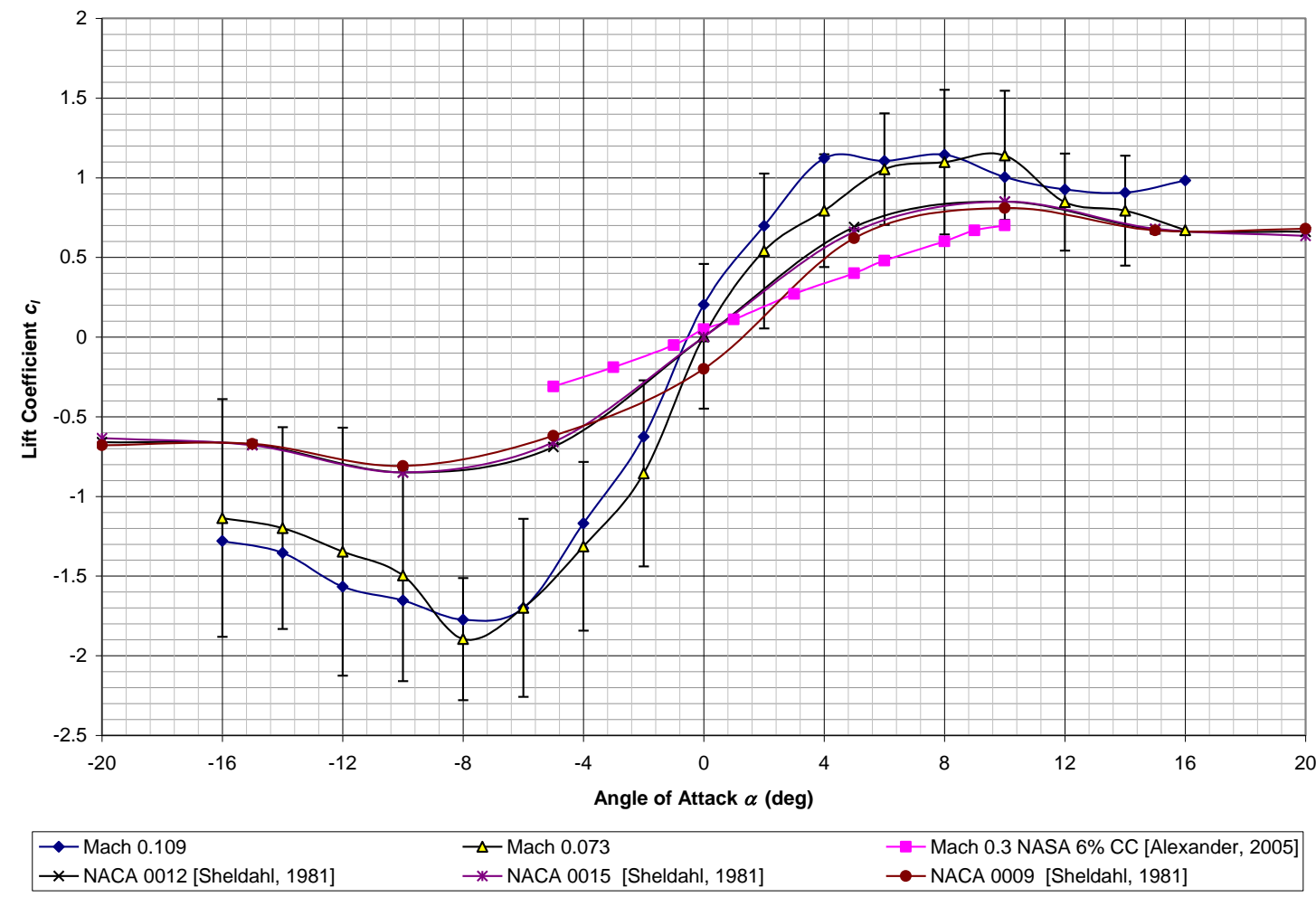

Figure 5.1: Lift Coefficient vs. Angle of Attack, No Blowing Comparison

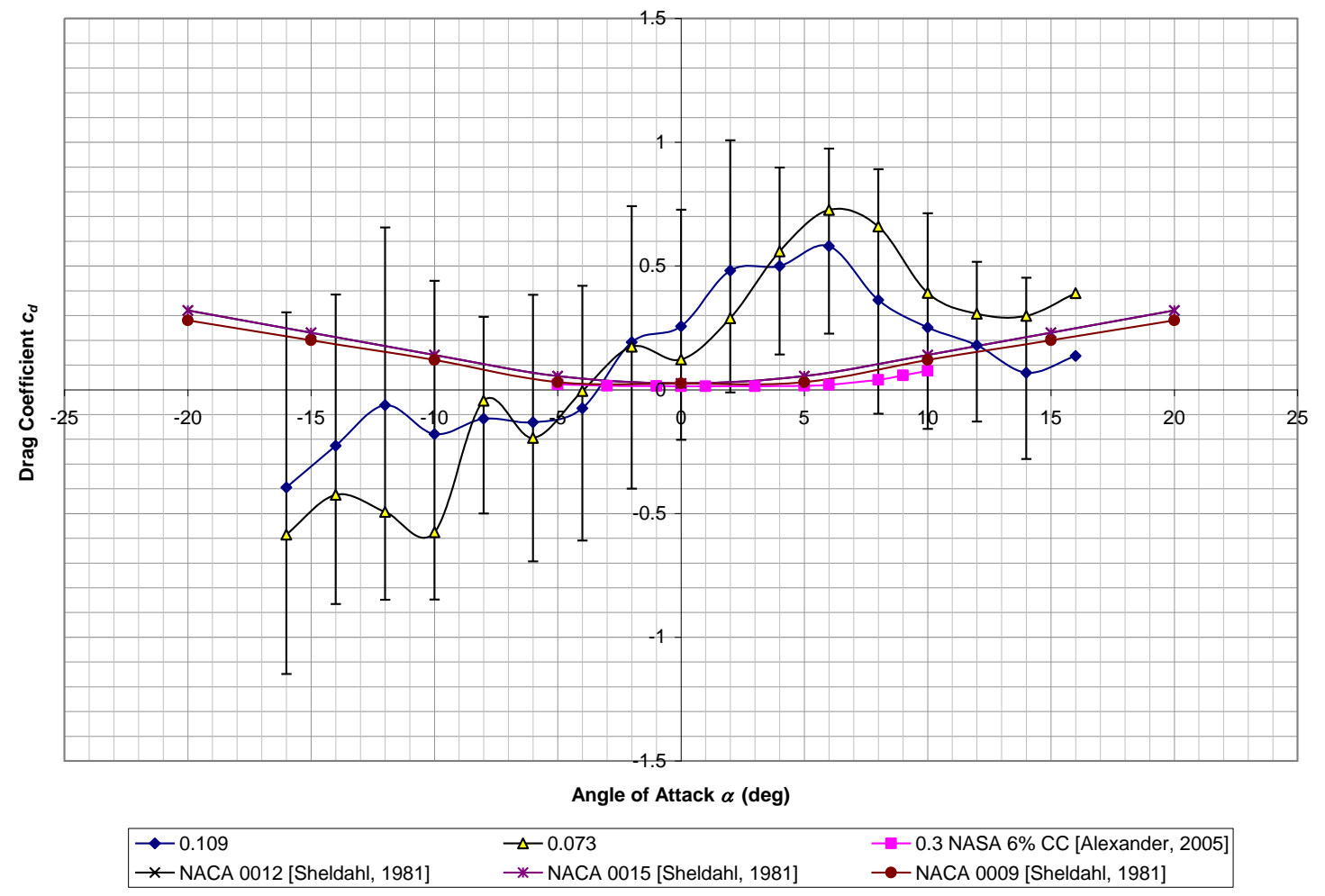

Figure 5.2: Drag Coefficient vs. Angle of Attack, No Blowing Comparison 


\subsection{Wind Tunnel Results}

The area of focus for this investigation was the effect of blowing on the aerodynamic characteristics of the airfoil in reverse flow. Since Mach number independence is assumed based on the previous section, three main parameters are of importance. They are the blowing coefficient (for both leading and trailing edges), the angle of attack (at significant points such as zero angle, small angles, and near-stall angles), and the change in the lift coefficient. For the actual values of the lift coefficients instead of the change in the values, see Appendix B. The following plots were constructed from subsets of the test ranges and show the general trending of the lift coefficients and the change in lift coefficients in the non-stall or near-stall regions only. More detailed graphical results are presented in Appendix B. Figure 5.3 through Figure 5.8 show the lift coefficient results with the trailing edge blowing coefficient constant and the leading edge blowing coefficient varying.

It is seen in Figure 5.4 and Figure 5.4 that at a 0 degree angle of attack, the introduction of leading edge blowing in reverse flow decreased the lift coefficient and this effect was most prominent at the blowing coefficient of approximately 0.0035 . At small positive angles of attack, the effect of the leading edge blowing was a slight decrease in the lift coefficient until a high blowing coefficient was achieved, at which time the effect became slightly positive. At positive near-stall conditions, the effect was a general increase in lift coefficient as the leading edge blowing coefficient was increased, while at negative near-stall conditions, the effect of the leading edge blowing was minimal. 


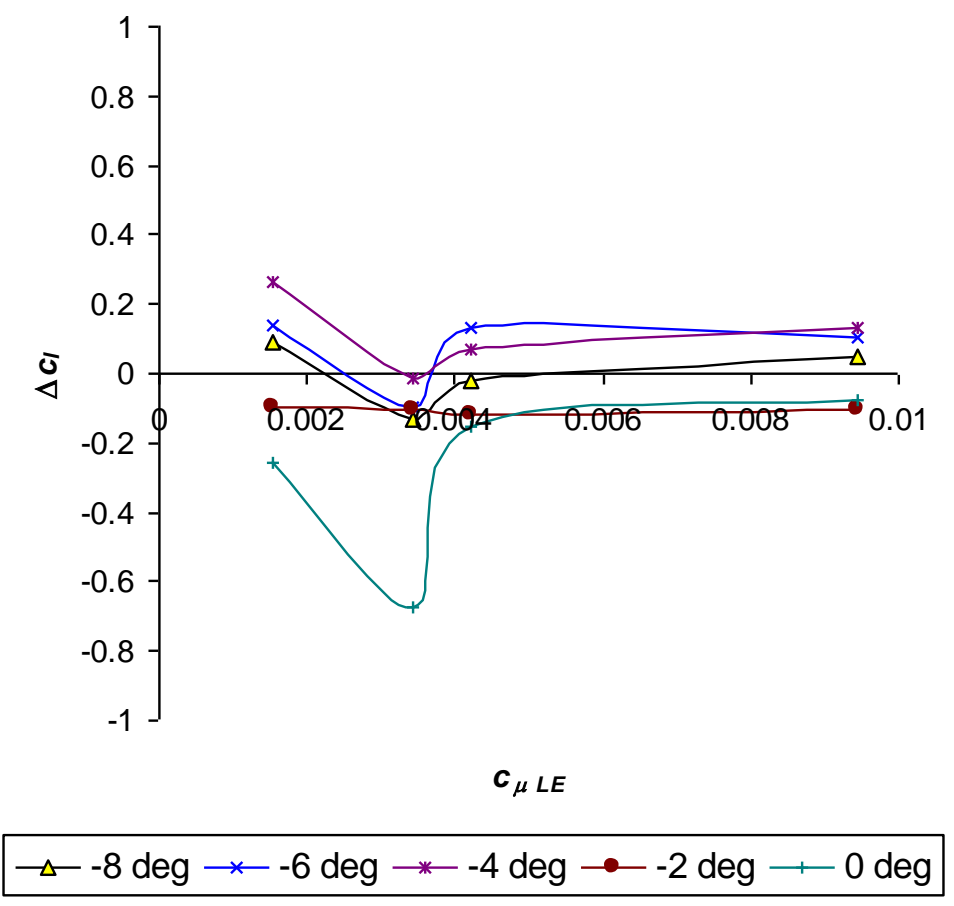

Figure 5.3: Change in Lift Coefficient vs. Leading Edge Blowing Coefficient vs. Negative Angle of Attack

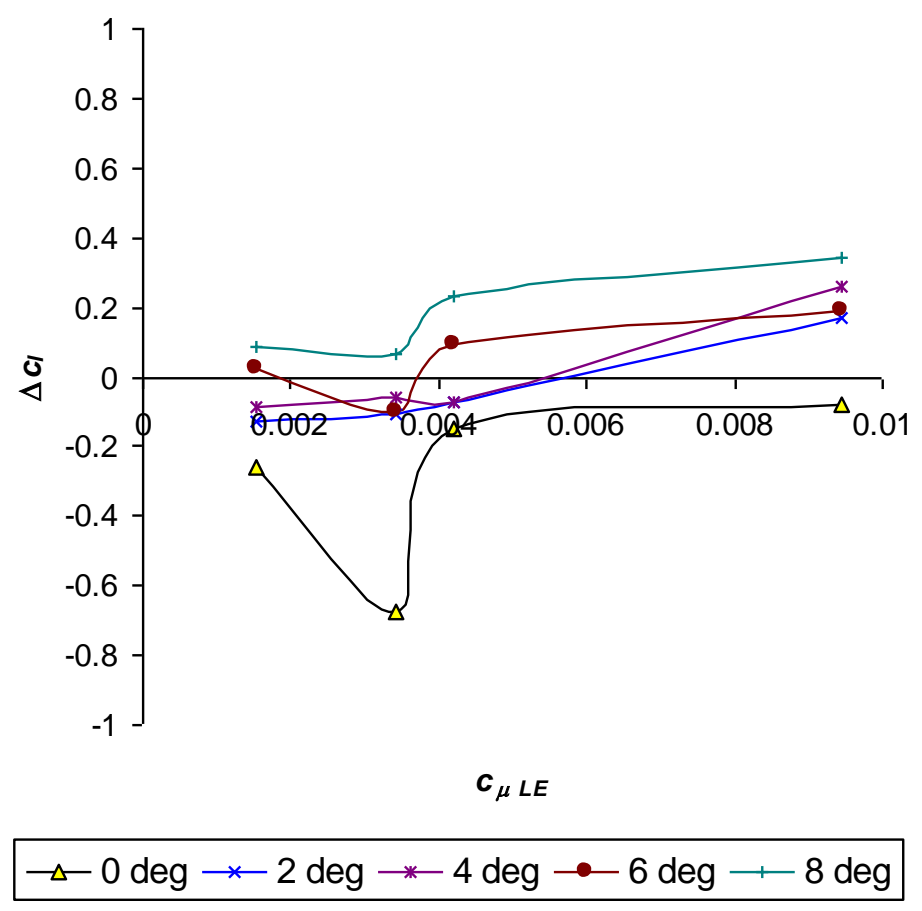

Figure 5.4: Change in Lift Coefficient vs. Leading Edge Blowing Coefficient vs. Positive Angle of Attack 
As seen in Figure 5.5 and Figure 5.6, adding in the trailing edge blowing at 10 psi increased the lift coefficient at negative near-stall conditions and decreased the lift coefficient at negative near-stall conditions. Otherwise, the effect was minimal.

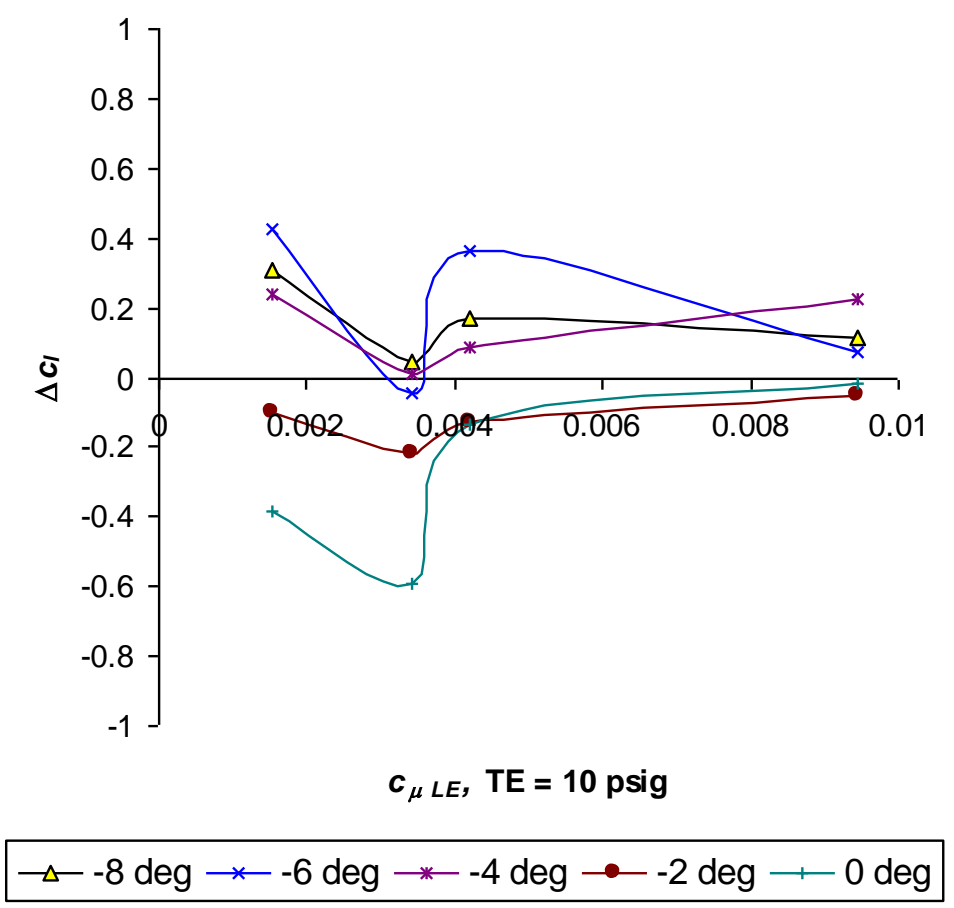

Figure 5.5: Change in Lift Coefficient vs. Leading Edge Blowing Coefficient vs. Negative Angle of Attack, Trailing Edge Blowing $=10$ psig 


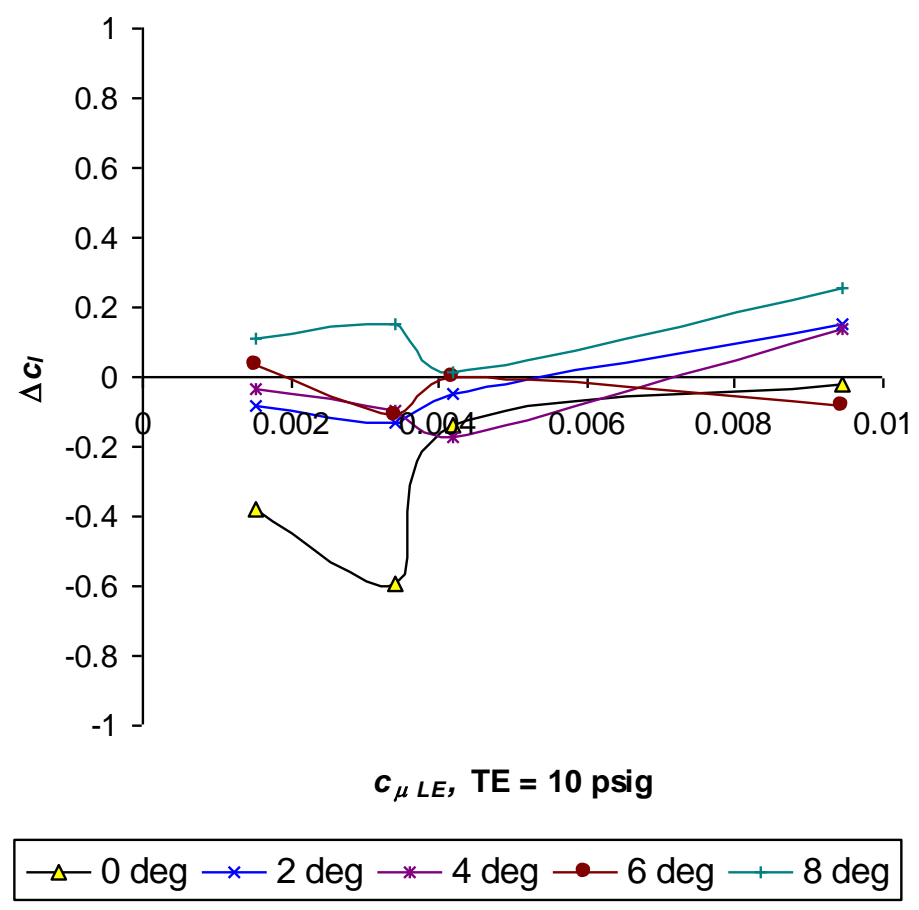

Figure 5.6: Change in Lift Coefficient vs. Leading Edge Blowing Coefficient vs. Positive Angle of Attack, Trailing Edge Blowing $=10$ psig

When the blowing coefficient on the trailing edge was increased, the effects are shown in Figure 5.7 and Figure 5.8 and are described as follows. At zero degrees angle of attack, the addition of more blowing to the trailing edge decreased the lift coefficient at low values of leading edge blowing and increased the lift coefficient at the higher values of leading edge blowing. At small positive angles of attack the lift coefficient decreased slightly but had less effect at the higher blowing rates for the leading edge. At small negative angles of attack, the lift coefficient had minimal effect at low blowing values for the leading edge but decreased the lift coefficient more as the blowing values of the leading edge increased. At the negative near-stall condition, this addition of trailing edge blowing increased the lift coefficient slightly over the less-blown similar condition. At near stall on the positive side, no appreciable effect was noted. 


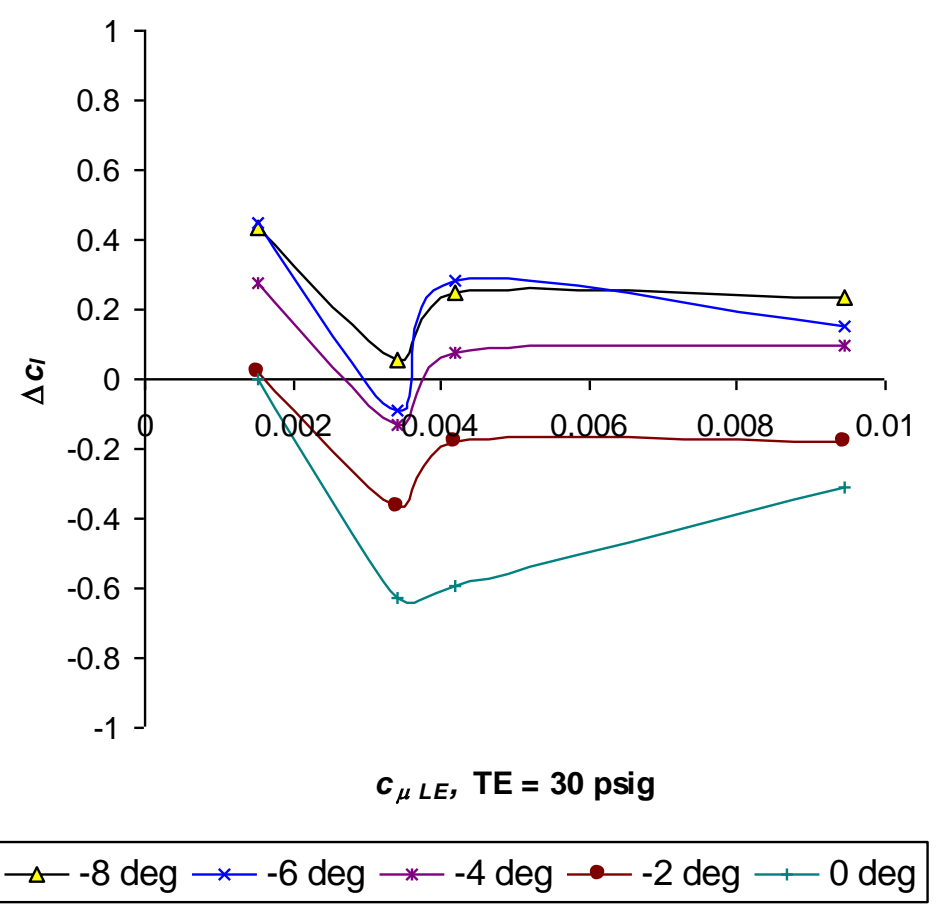

Figure 5.7: Change in Lift Coefficient vs. Leading Edge Blowing Coefficient vs. Negative Angle of Attack, Trailing Edge Blowing $=30$ psig

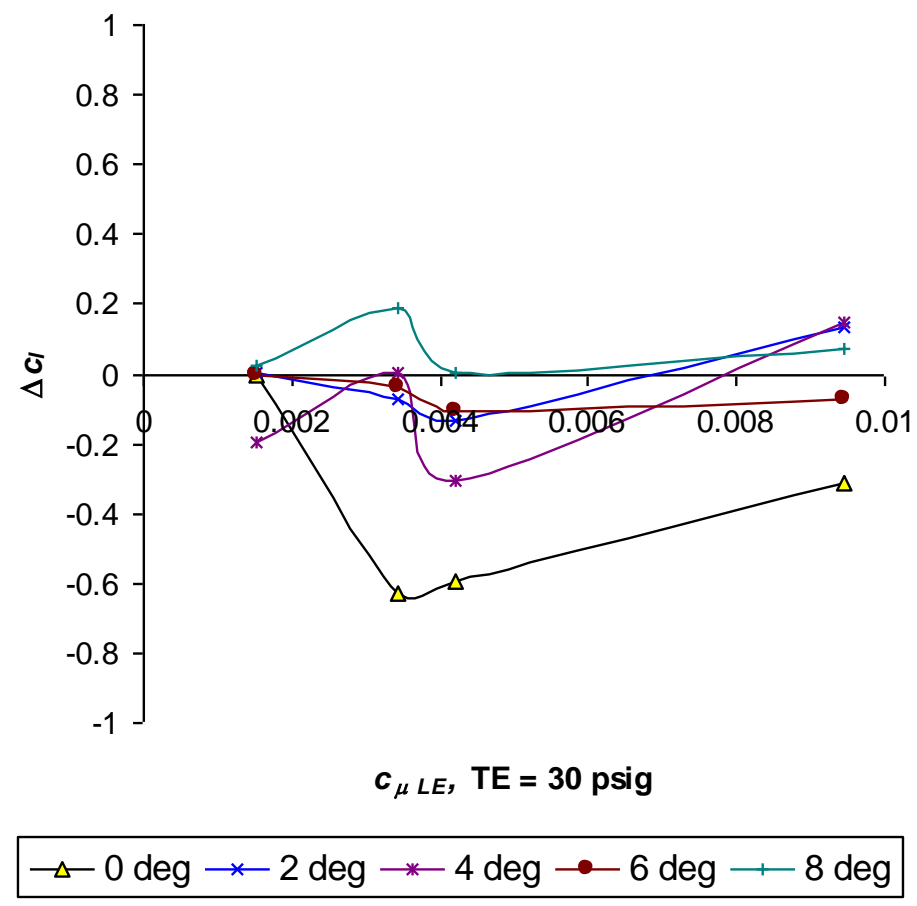

Figure 5.8: Change in Lift Coefficient vs. Leading Edge Blowing Coefficient vs. Positive Angle of Attack, Trailing Edge Blowing $=30$ psig

Figure 5.9 through Figure 5.14 show the lift coefficient results with the leading edge blowing constant and the trailing edge blowing constant. As shown in Figure 5.10, the effect of the trailing-edge-only blowing maintained 
a similar general shape, with the exception of the -8 deg curve, where an at-stall or after-stall effect is presumed. The plot shows that at low rates, the lift coefficient is decreased for all angles of attack. At trailing edge blowing coefficient of approximately 0.003 , the lift coefficient is increased for all angles of attack. At medium to higher blowing rates, the lift coefficient decreases for angles of attack from 0 to 8 degrees. Also, at medium to higher blowing rates, the small negative angle of attack condition increases the lift coefficient, but less than at the 0.003 trailing edge blowing rate.

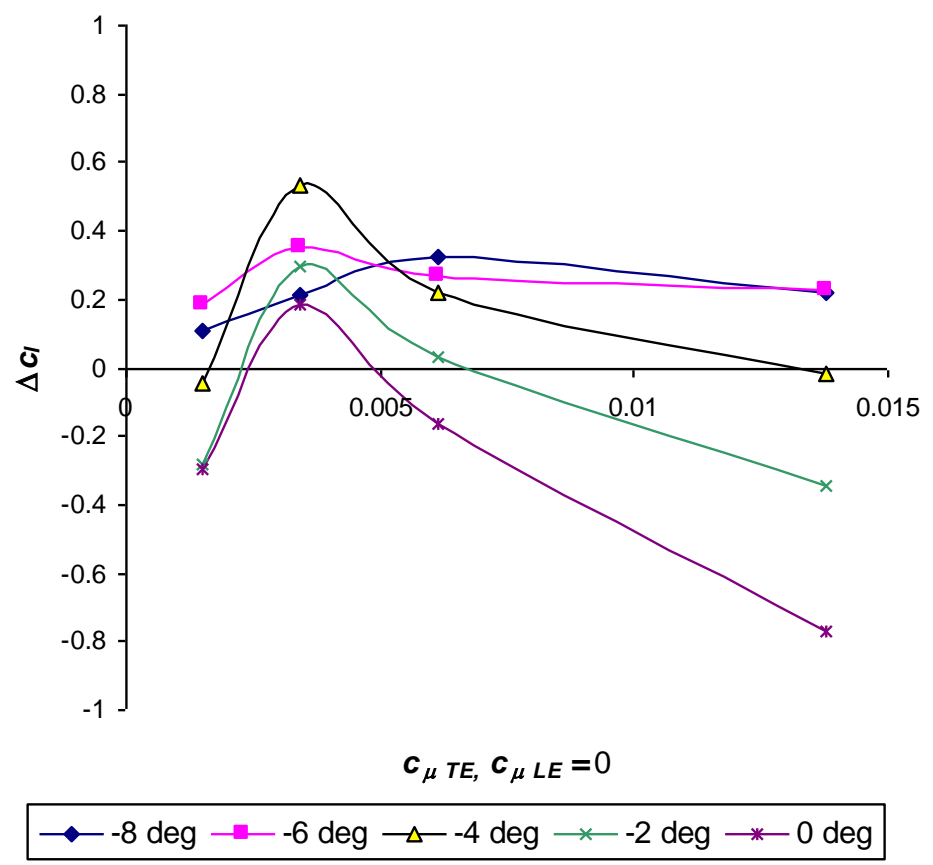

Figure 5.9: Change in Lift Coefficient vs. Trailing Edge Blowing Coefficient vs. Negative Angle of Attack

When the leading edge blowing was added, the entire general trend for the lift coefficient changed as shown in Figure 5.11 and Figure 5.12. The zero degrees to the negative near stall angles of attack change in lift coefficient trends were similar, as the small and near positive stall angles of attack were similar in trends. There existed a large decrease in the lift coefficient with the angle of attack was zero, especially at the lower blowing rates for the leading edge blowing coefficient. Also, an increase in the lift coefficient at negative small angles of attack to the near stall conditions for angles of attack was observed at high vales of the leading edge blowing coefficient. When the angle of attack was a small positive value, the lift coefficient slightly decreased for all leading edge blowing values.

Finally, the further addition of blowing to the leading edge restored the lift coefficient general trend shapes back to the no-blowing condition shapes. This can be seen in Figure 5.13 and Figure 5.14. The magnitudes of these shapes were lower than the no blowing condition and had one exception: the small positive angle of attack condition. Under this condition, the general trend seemed to "mix" the non-blown condition with the low-blown condition. 


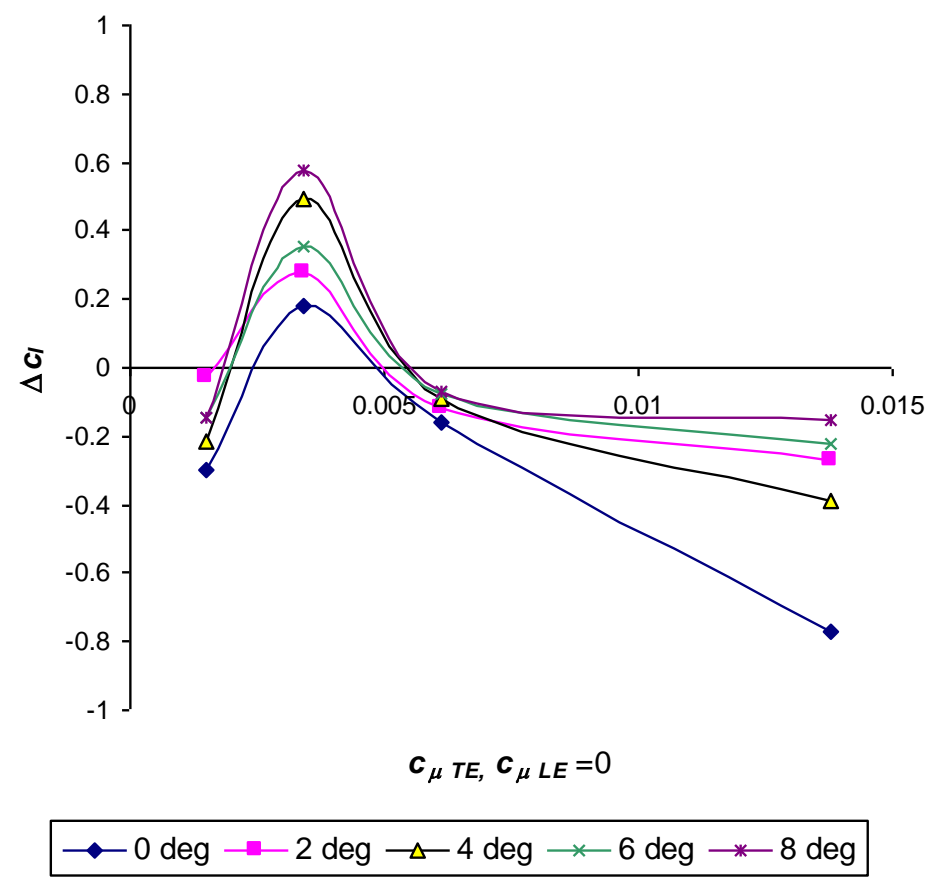

Figure 5.10: Change in Lift Coefficient vs. Trailing Edge Blowing Coefficient vs. Positive Angle of Attack

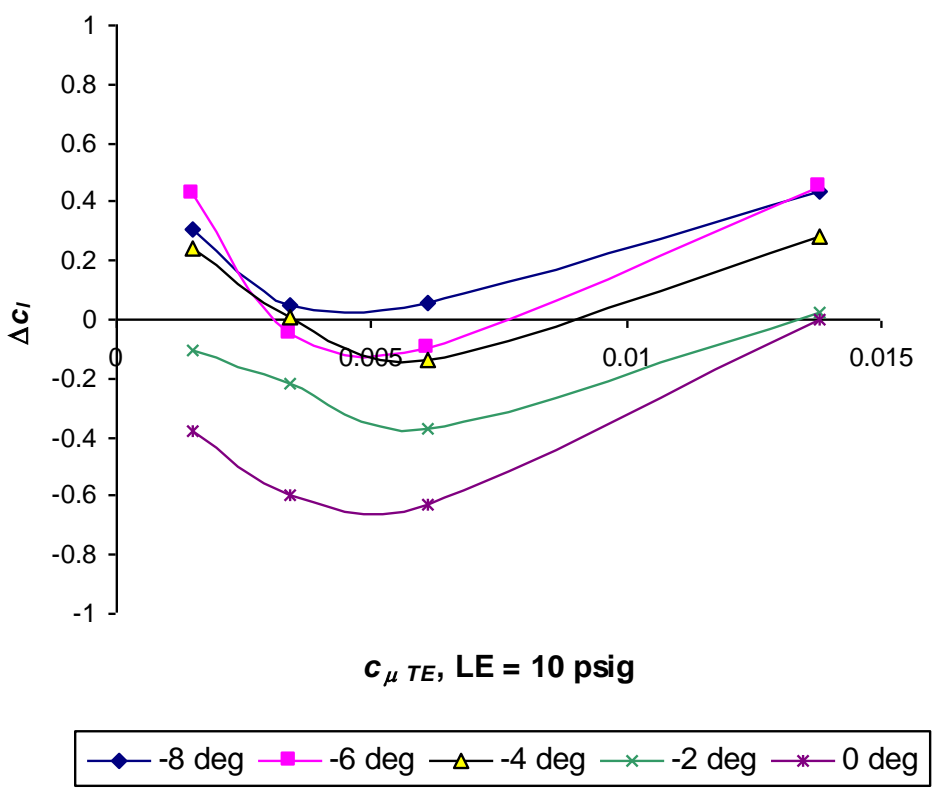

Figure 5.11: Change in Lift Coefficient vs. Trailing Edge Blowing Coefficient vs. Negative Angle of Attack, Leading Edge Blowing = 10 psig 


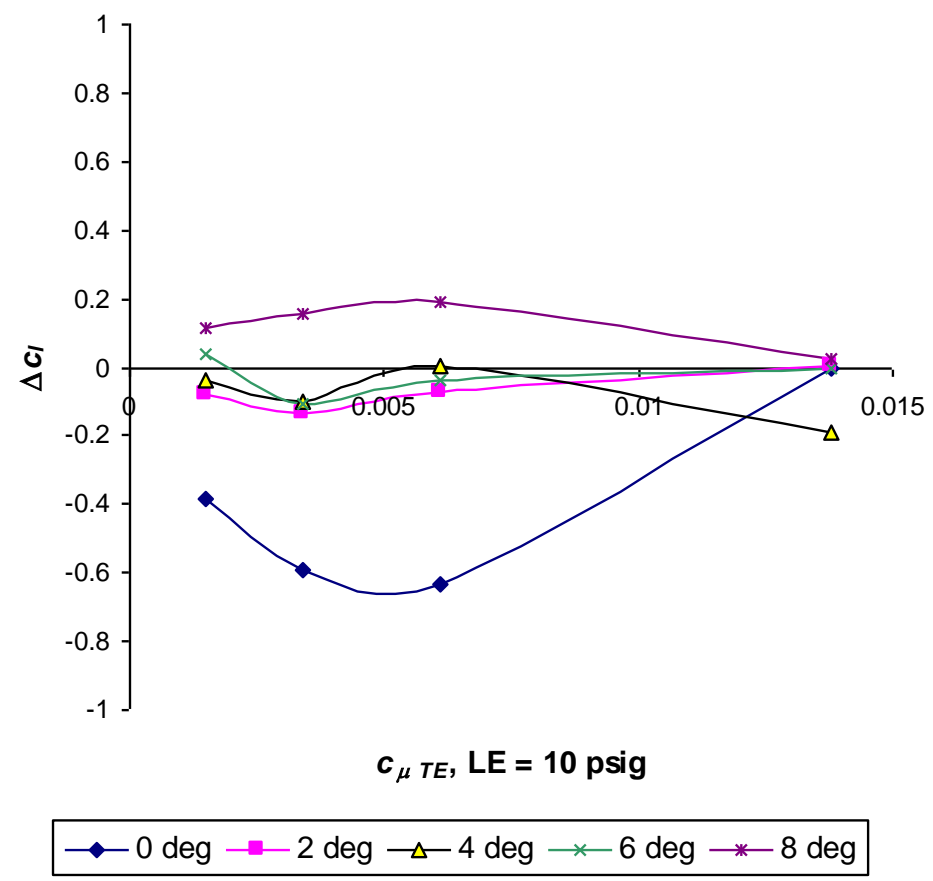

Figure 5.12: Change in Lift Coefficient vs. Trailing Edge Blowing Coefficient vs. Positive Angle of Attack, Leading Edge Blowing $=10$ psig

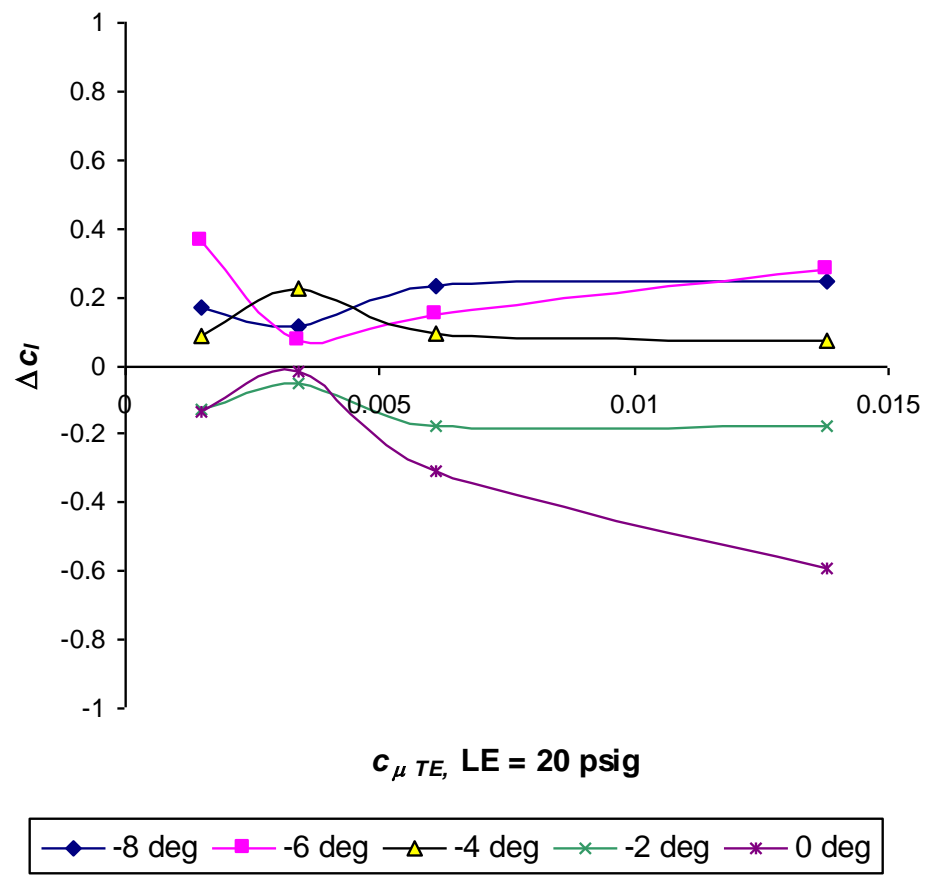

Figure 5.13: Change in Lift Coefficient vs. Trailing Edge Blowing Coefficient vs. Negative Angle of Attack, Leading Edge Blowing $=20$ psig 


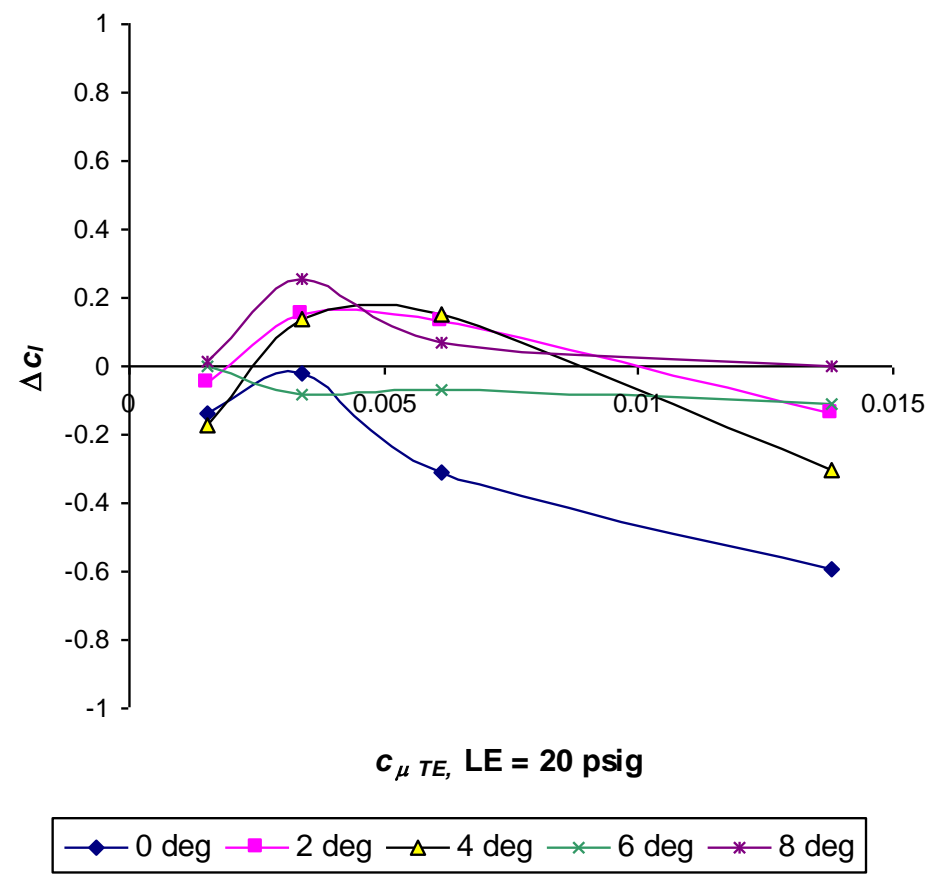

Figure 5.14: Change in Lift Coefficient vs. Trailing Edge Blowing Coefficient vs. Positive Angle of Attack, Leading Edge Blowing $=20$ psig

Table 5.1 and Table 5.2 show the summarized results in a tablular format. This is useful for determining which blowing configuration(s) would produce the desired effects in reverse flow. For example, if the desired condition was to increase the lift (or decrease the negative lift) at a small negative angle of attack in reverse flow, the configuration to best fit the scenario would be to blow on the trailing edge only at a low to medium blowing rate. This selection is based on the fact that the lowest amount of blowing necessary would produce the most aerodynamic

efficiency. 
Table 5.1: Summarized Effects of Various Blowing Configurations in Reverse Flow, Leading or Trailing Edge Blowing Only

\begin{tabular}{|l|l|c|c|c|c|c|}
\hline \multicolumn{2}{|c|}{ Blowing } & \multicolumn{5}{c|}{ Reverse Angle of Attack } \\
\hline \multirow{4}{*}{$\begin{array}{c}\text { Configuration } \\
\text { Beading Edge }\end{array}$} & Amount $c_{\mu}$ & Zero & Small Neg & Large Neg & Small Pos & Large Pos \\
\cline { 2 - 7 } & Low & - & 0 & + & - & + \\
\cline { 2 - 7 } & Low-Med & - & 0 & - & - & 0 \\
\cline { 2 - 7 } & High & - & 0 & + & - & + \\
\hline \multirow{4}{*}{$\begin{array}{c}\text { Trailing Edge } \\
\text { Blowing Only }\end{array}$} & Low & - & - & + & - & + \\
\cline { 2 - 7 } & Led & - & + & + & - & + \\
\cline { 2 - 7 } & High & - & - & + & - & - \\
\hline
\end{tabular}

Table 5.2: : Summarized Effects of Various Blowing Configurations in Reverse Flow, Combined Blowing

\begin{tabular}{|c|c|c|c|c|c|c|}
\hline \multicolumn{2}{|c|}{ Blowing } & \multicolumn{5}{|c|}{ Reverse Angle of Attack } \\
\hline Configuration & Amount $c_{\mu}$ & Zero & Small Neg & Large Neg & Small Pos & Large Pos \\
\hline \multirow{4}{*}{$\begin{array}{l}\text { Leading Edge } \\
\text { Varying, } \\
\text { Trailing Edge } \\
=10 \mathrm{psi}\end{array}$} & Low & - & 0 & + & - & + \\
\hline & Low-Med & - & - & 0 & - & 0 \\
\hline & Med & - & 0 & + & - & 0 \\
\hline & High & 0 & + & + & + & + \\
\hline \multirow{4}{*}{$\begin{array}{l}\text { Leading Edge } \\
\text { Varying, } \\
\text { Trailing Edge } \\
=30 \mathrm{psi}\end{array}$} & Low & 0 & + & + & - & 0 \\
\hline & Low-Med & - & - & 0 & 0 & + \\
\hline & Med & - & 0 & + & - & 0 \\
\hline & High & - & 0 & + & + & 0 \\
\hline \multirow{4}{*}{$\begin{array}{l}\text { Trailing Edge } \\
\text { Varying, } \\
\text { Leading Edge } \\
=10 \mathrm{psi}\end{array}$} & Low & - & - & 0 & - & + \\
\hline & Low-Med & - & - & + & - & + \\
\hline & Med & - & - & + & - & + \\
\hline & High & 0 & 0 & + & 0 & 0 \\
\hline \multirow{4}{*}{$\begin{array}{l}\text { Trailing Edge } \\
\text { Varying, } \\
\text { Leading Edge } \\
=20 \mathrm{psi} \\
\end{array}$} & Low & - & 0 & + & - & 0 \\
\hline & Low-Med & + & + & + & + & + \\
\hline & Med & - & + & + & + & + \\
\hline & High & - & 0 & + & - & - \\
\hline
\end{tabular}




\section{Chapter 6: Conclusions}

It is concluded that the potential exists for increasing forward flight speeds for helicopters using circulation control in the reverse flow regions of the helicopter rotor environment. Specifically, it is concluded that positively altering the aerodynamic characteristics, primarily the lift coefficient, in reverse flow, is possible through circulation control.

Through this investigation, the general trends were found for the aerodynamic characteristics of a 10:1 circulation controlled elliptical airfoil in reverse flow. These trends led to the selection of blowing configurations to decrease the asymmetric loading condition based on the condition of the local blade environment. The data generated for this experiment can be used for implementation into a circulation controlled rotor performance simulation program or to generate a preliminary testing schedule for a spinning rotor experiment.

Within the wind tunnel data, Mach number independence was successfully achieved within the limits of uncertainty. It is also concluded that the magnitude of the lift and drag coefficients are large in comparison with previous experiments. These discrepancies are due to the large and inconsistent tare values in the angle of attack apparatus.

It is concluded from the general trends from Figure 5.4 to Figure 5.8 that the leading edge in reverse flow alters the lift coefficient similarly to a trailing edge Coanda surface in forward flow. From Figure 5.4 and Figure 5.4, the lift coefficient increases at positive angles of attack. However, at zero angle of attack, the lift coefficient is decreased, possibly due to the fact that the blowing slot is not tangential to the free stream flow, hence causing separation. At negative angles of attack, the flow seems only slightly influenced by the leading edge blowing. It is observed from Figure 5.5Figure 5.6 that adding 10 psi trailing edge blowing pressure decreases the lift coefficient at positive angles of attack but increases the lift coefficient at zero and negative angles of attack. It is also observed from Figure 5.7Figure 5.8 that adding an additional 20 psi to the trailing edge blowing pressure decreases the lift coefficient at small negative, zero, and positive angles of attack, but increases the lift coefficient at near negative stall conditions.

It is concluded from observing the general trends from Figure 5.9 through Figure 5.14 that the trailing edge in reverse flow behaves similarly to a leading edge Coanda surface in forward flow. From Figure 5.9 and Figure 5.10, the lift coefficient is decreased at zero and positive angles of attack, and increased at negative angles of attack. The large peak in this figure could be attributed to a complex separation or attachment of the flow or due to a data anomaly. It is observed from Figure 5.11and Figure 5.12 that the addition of leading edge blowing at 10 psi reverses the change in lift coefficient trend. It is observed from Figure 5.14 that original trend from Figure 5.10 is restored with the addition of another 10 psi to the leading edge. 


\section{Chapter 7: Recommendations}

This section describes the author's recommendations for further experimentation. It is recommended to expand the scope of this experiment, based on the positive results obtained, and validate with computational and numerical methods.

For a future experiment, that will validate and increase the scope and detail of this investigation, it is recommended to create a new, redesigned support structure that will have a rigid base and tight tolerances to minimize deflection. It is recommended that the plate system to change the angle of attack be a design with low manufacturing tolerances, lightweight materials, full automation, and the ability to rotate an entire 360 degrees. This design will increase accuracy, precision, and allow for dynamic studies. Specifically, the experimenter could study the dynamic effects of the blowing actuation and fast blade pitch change. The new design should have low manpower and time requirements for testing, ideally allowing for one-person operation.

To further investigate the flow structure and the fluid mechanics associated with circulation control in reverse flow, it is recommended that the next model have low manufacturing tolerances (for the jet slots and overall geometry) and include at least one thermocouple and one pressure sensor inside each blowing plenum. This will allow for the conventional measurement procedure (see Alexander and Anders, 2005) for the determination of the jet exit velocity. It is also recommended that the internal design be designed to include several pressure taps (see Englar and Williams, 1975) to allow the pressure integration method to be used for the pressure coefficient calculation, center of pressure calculation, and the determination of the lift and drag forces. Implementation of this method will also allow for the calculation of the aerodynamic moment, which, in turn, will allow the application of further tunnel wall corrections.

A recommendation for the next generation air supply system is that temperature and pressure measurement locations be placed at a critical venturi similar to Alexander and Anders, 2005 on each supply line to measure mass flow through the piping with high accuracy. A high output compressor could increase the blowing coefficient range and reduce downtime between runs.

Another recommendation is to create and use a data acquisition system that includes measurements of the mass flow, jet exit velocities, internal model sensors, and an angle of attack sensor at every time step or run. This system should also include calibration and gain matrix algorithms to allow for real-time generation of lift and drag coefficient plots. 


\section{Chapter 8: References}

Angle, G., “Aerodynamic Benefits of Near-Surface-Actuated Circulation Control Blowing Slots for Rotorcraft Use,” Ph.D. Dissertation, West Virginia University, Morgantown, WV, 2008

Kweder, Jonathan, "Effect of Circulation Control Blowing Slots on Stall Angles of a 10:1 Elliptical Airfoil," Masters Thesis, West Virginia University, Morgantown, WV, 2008

Johnson, Wayne. Helicopter Theory. Dover Publications, Inc., New York, 1980, pp. 152,153,198

Critzos, Chris C., Heyson, Harry H., Boswinkle Jr., Robert W., "Aerodynamic Characteristics of NACA 0012 Airfoil Section at Angles of Attack from 0 to 180o," NACA Report TN 3361, 1955

Wood, N.J., "The Aerodynamics of Circulation Control Aerofoils," Ph.D. Thesis, Department of Aeronautics and Astronautics, Stanford University, Stanford, California, July 1981

Novak, C.J.; Cornelius, K.C.; Road, R.K.: Experimental Investigations of Circular Wall Jet on a Circulation Control Airfoil. AIAA 87-0155, Jan. 1987.

Bertin, J.J., Aerodynamics for Engineers, $4^{\text {th }}$ ed., Prentice Hall, Upper Saddle River, New Jersey, 2002. pp. 513

Cheesman, I.C.; and Seed, A.R.: The Application of Circulation Control by Blowing to Helicopter Rotors. Journal of Royal Aeronautical Society, Vol. 71, No. 848, July 1966.

Englar, R.J.: Two Dimensional Transonic Wind Tunnel Tests of Three 15-Percent -Thick Circulation Control Airfoils. Naval Ship R\&D Center Report ASED-182 (AD882-075), December 1970

Mavis, D.N.; Kirby, M.R.: Takeoff/Landing Assessment of an HSCT with Pneumatic Lift Augmentation. AIAA-990534, Jan. 1999

Abramson, J.: The Low Speed Characteristics of a 15-Percent Quasi-Elliptical Circulation Control Airfoil with Distributed Camber. David W. Taylor Naval Ship R\&D Center Report DTNSRDC/ASED-79/07(AD-A084-176), May 1979

Young, D.W., Zonars D., Wind tunnel tests of the Coanda wing and nozzle. U.S.A.F. TR 6199, September 1950.

Alexander, M., Anders, S., et. Al. "Trailing Edge Blowing on a Two-Dimensional Six-Percent Thick Elliptical Circulation Control Airfoil Up to Transonic Conditions” NASA TM-2005-213545. Langley, VA, 2005

Launder, B.E., Rodi, W., Summary Presentation of the Turbulent Wall Jet - A Review of the Experimental Data Presented at the AFOSR-HTTM-Stanford Conference on Complex Turbulent Flows. Stanford University, September 1980

Gartshore, I.S., Newman, B.G., The Turbulent Wall Jet in an Arbitrary Pressure Gradient. The Aeronautical Quarterly, February 1969.

Kruka, V., Eskinazi, S., The Wall Jet in a Moving Stream. Journal of Fluid Mechanics (1964), Volume 20 , Part 4.

Ramaprian, B.R. Shivaprasad, B.G., "Mean Flow Measurements in Turbulent Boundary Layers Along Mildly Curved Surfaces," AIAA Journal Vol. 15 No. 2, February 1977, pp. 189-196

Ramaprian, B.R., Shivaprasad, B.G., "Turbulent Measurements in Boundary Layers Along Mildly Curved Surfaces," Journal of Fluids Engineering - Transactions of ASME Vol. 100 No. 1, March 1978, pp. 37 -46 
Gibbs, E.J., "Analysis of Circulation Controlled Airfoils," Ph.D. Dissertation, Department of Aerospace Engineering, West Virginia University, Morgantown, WV, 1975.

Newman, B.G., "The Deflection of Plane Jets by Adjacent Boundaries - Coanda Effect," Boundary Layer and Flow Control, Pergamon Press Vol. 1, 1961, p232.

Wilson, D.J., Goldstein, R.J., Turbulent Wall Jets with Cylindrical Stream-wise Surface Curvature. Journal of Fluids Engineering, September 1976

Dash, S. M., York, B. J., Sinha, N., Dvorak, F. A., "Wall Jet Analysis for Circulation Control Aerodynamics, Part I: Fundamental CFD and Turbulence Modeling Concepts," Proceedings of the 1986 Circulation Control Workshop, NASA CP-2432, February 1986, pp.23-69

Kind, R. J., Maull, D. J., “An Experimental Investigation of a Low-Speed Circulation-Controlled Aerofoil," The Aeronautical Quarterly, Vol. XIX, May 1968, pp. 170-182.

Ambrosiani, J.P., "Analysis of a Circulation Controlled Elliptical Airfoil," Ph. D. Dissertation, Department of Aerospace Engineering, West Virginia University, Morgantown, WV, 1971

Englar, R.J., Williams, R.M., Test Techniques for High-Lift, Two-Dimensional Airfoils with Boundary Layer and Circulation Control for Application to Rotary Wing Aircraft. NSRDC 4645, July 1975.

Rogers, E.O, Donnelly, M.J., Characteristics of a Dual-Slotted Circulation Control Wing of Low Aspect Ratio Intended for Naval Hydrodynamic Applications. AIAA 2004-1244

Barlow, J.B., Rae Jr., W.H., Pope, A., Low Speed Wind Tunnel Testing, $3^{\text {rd }}$ Edition, John Wiley \& Sons, Inc., New York, 1999.

Allen, H.J., and Vincenti, W.G., "Wall Interference in a Two-Dimensional-Flow Wind Tunnel with Consideration of the Effect of Compressibility," NACA TR 782, 1944.

Abbot, Ira H., and Von Doenhoff, Albert E., Theory of Wing Sections, 2nd ed., Dover, New York, 1949, Appendix IV.

Angle, G., et. Al, "Circulation Controlled Fixed-Root Helicopter Concept”, AHS Forum 62 Proceedings, Grapevine, TX, 2006

Lyons, Michael J., et al, "Deflection and Vibration Analysis of a Wind Tunnel Test Stand," AIAA Aerodynamic Measurement Technology and Ground Testing Conference, Doubletree Hotel Seattle Airport, Seattle, WA, 2008

Anderson, John D., Fundamentals of Aerodynamics, 2nd ed., McGraw-Hill, New York, 1991, pp. 76

Mills, A.F., Basic Heat and Mass Transfer, 1st ed., Richard D. Irwin, Inc., Illinois, 1995, pp. 851

Anderson, John D., Fundamentals of Aerodynamics, 2nd ed., McGraw-Hill, New York, 1991, pp. 190

Jones, F.E., Techniques and Topics in Flow Measurements, CRC Press, Boca Raton, FL, 1995.

Fury, Rudolf J., "National Geodetic Survey (NGS) Gravity Prediction Methodology," [online article], URL: http://www.ngs.noaa.gov/TOOLS/Gravity/grav_method.htm [cited 17 June 2008].

Jekeli, C., "Hardy's Multiquadric-Biharmonic Method for Gravity Field Predictions," Computers Mathematical Applications, 1994, Vol. 28, No. 7, pp 43-46

Staff of Ames Research Center, "Equations, Tables, and Charts for Compressible Flow." NACA Report 1135, 1953 
Beckwith, T.G., Marangoni, R.D., Lienhard V, J.H., Mechanical Measurements, $5^{\text {th }}$ ed., Addison-Wesley Publishing Company, Inc., Reading, MA, 1995

Sheldahl, Robert E., and Klimas, Paul C., "Aerodynamic Characteristics of Seven Symmetrical Airfoil Sections Through 180-Degree Angle of Attack for Use in Aerodynamic Analysis of Vertical Axis Wind Turbines," National Technical Information Services SAND80-2114, 1981. 


\section{Appendix A - Load Cell Calibration and Force Calculation Experimentation}

This appendix describes, in detail, the procedure and methodology used to calibrate the system of load cells used in this experiment and calculate the forces used for input into the lift and drag coefficient equations. This section also compares different methods of determining the forces and compares their associated errors.

\section{A.1 Load Cell Calibration}

Four S-beam type load cells and were calibrated with the model in place in the wind tunnel. Two load cells were attached at the top and bottom of the sting: one in the freestream (drag) direction, and one in the freestreamnormal (lift) direction as shown in Figure 4.7. Forces were applied in the positive and negative direction in each load cell axis and recorded. These forces were applied to the top load cells through the use of a pulley system and a calibrated weight set as shown in Figure A.1. Similarly, the bottom load cells were weighted. The calibration testing scheme was one of random force application to minimize loading bias. The definitions for the calibration testing scheme are shown in Table A.1 and Table A.2. The calibration testing schedule is shown in Table A.3.

Table A.1: Description of Calibration Testing Schedule Terms

\begin{tabular}{|c|c|}
\hline Load & Description \\
\hline PBL & Positive Bottom Lift \\
\hline NBL & Negative Bottom Lift \\
\hline PBD & Positive Bottom Drag \\
\hline NBD & Negative Bottom Drag \\
\hline PTL & Positive Top Lift \\
\hline NTL & Negative Top Lift \\
\hline PTD & Positive Top Drag \\
\hline NTD & Negative Top Drag \\
\hline
\end{tabular}

Table A.2: Calibration Load Definitions

\begin{tabular}{|c|c|}
\hline Load & Acronym \\
\hline Light & $\mathrm{L}$ \\
\hline Med & $\mathrm{M}$ \\
\hline Heavy & $\mathrm{H}$ \\
\hline $\begin{array}{c}\text { Extra } \\
\text { Heavy }\end{array}$ & $\mathrm{XH}$ \\
\hline Zero & $\mathrm{Z}$ or 0 \\
\hline
\end{tabular}


Table A.3: Calibration Testing Schedule for Single Loads

\begin{tabular}{|c|c|c|c|c|}
\hline Load & \multicolumn{4}{|c|}{ Single Testing Schedule } \\
\hline PBL & Med & Light & Heavy & Zero \\
\hline PBD & Heavy & Light & Med & Zero \\
\hline NBL & Light & Heavy & Med & Zero \\
\hline NBD & Med & Heavy & Light & Zero \\
\hline PTL & Med & Light & Heavy & Zero \\
\hline PTD & Heavy & Light & Med & Zero \\
\hline NTL & Light & Heavy & Med & Zero \\
\hline NTD & Med & Heavy & Light & Zero \\
\hline
\end{tabular}

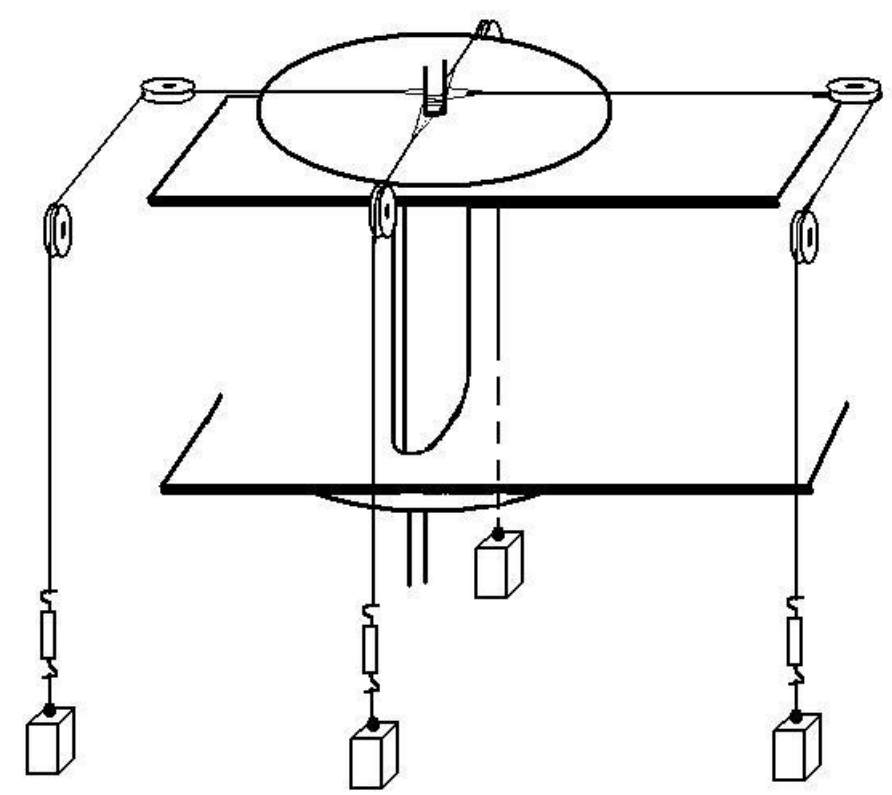

Figure A.1: Calibration Setup for Top Load Cells

The calibration data collection was performed using a PC and the custom data acquisition hardware and software associated with the low-speed closed-loop wind tunnel described previously. Each test point on the testing schedule was performed using a sampling rate of $1000 \mathrm{~Hz}$ for a 3 second time period.

Figure A.2 shows this using sample 7-point calibration curves (top lift applied force only) along with the corresponding regression equations generated by custom MATLAB programs. For example, Figure A.2 shows the voltage readings of each of the four load cells in response to the applied forces from the calibrated weight set at the top location in the lift direction. This allows a regression equation to be fit for each load cell and used for the force 
calculations.
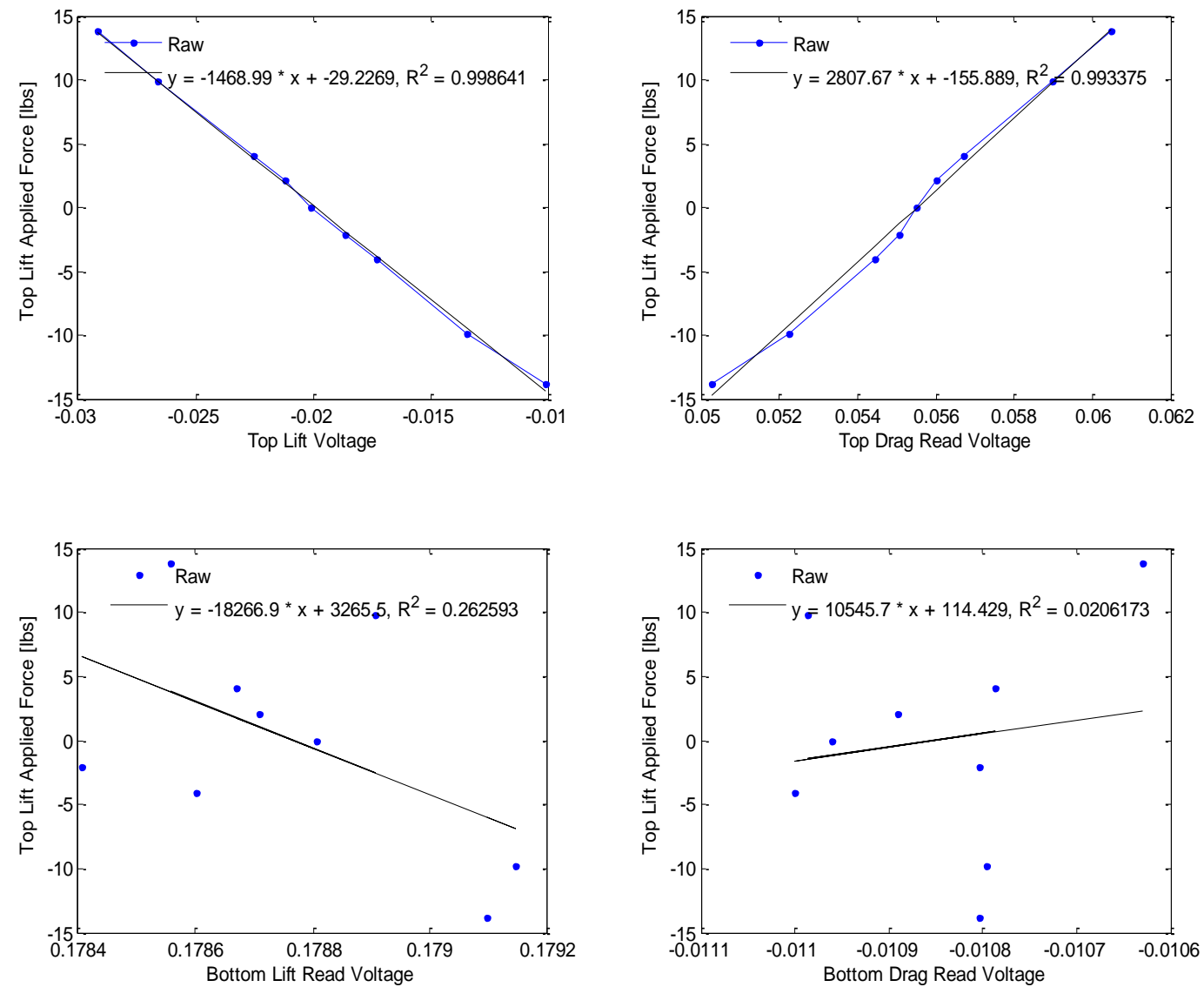

Figure A.2: Top Lift loaded Calibration Curves

From the visual inspection of the calibration plots, it is concluded that only the at-location (top or bottom) on-axis and off-axis deflections contribute significantly to the force calculation. The $\mathrm{R}^{2}$ values from the regressions suggest further that the on-location sensors are highly correlated to the force measurements whereas the off-location sensors may not be correlated. The best method for the calibration and data reduction is the inclusion of all the parameters in the gain matrix and provides significant improvement over the linear 7-point on-location on-axis regression equations. It is also concluded that the force calculation method is validated for multiple-loading configurations, retaining similar error values.

\section{A.2 Preliminary Force Calculation}

The 3,000 samples of voltage per test point were first averaged and the standard deviations calculated using a custom MATLAB program. These test points were then separated into four categories (columns). 
- Top Lift (TL) Loaded, All others unloaded

- Top Drag (TD) Loaded, All others unloaded

- $\quad$ Bottom Lift (BL) Loaded, All others unloaded

- Bottom Drag (BD) Loaded, All others unloaded

Each category contains voltage readings for each of the four channels (TL, TD, BL, and BD) for the various loadings. The data was then plotted using the same categories. For example, each channel was recorded with only varying the load applied in the top lift direction. A linear regression was then fit using custom MATLAB programs provided in Appendix D. A 7-point calibration was used to increase accuracy in the equations produced by each linear squares regression.

Since it was not known which method of calibration would produce the best results, a simple experiment was set up. First, the on-axis regression equations from the calibration procedure were used to calculate the forces as described by Equation (A.1), which expanded is Equation (A.2).Once these forces were calculated, the known values of the suspended weights were subtracted from calculated values to find the error as described by Equation (A.3). Next, the max and average values of the error within the four channels were found. Then, the maximum error value of the set of maximum error values and the average error value of the set of average error values over the many test points were found. The results give a quantitative value of the accuracy of the calibration method.

$$
\begin{aligned}
& \{F\}=[A]\{V\}+[B] \\
& \left\{\begin{array}{l}
F_{T L} \\
F_{T D} \\
F_{B L} \\
F_{B D}
\end{array}\right\}=\left[\begin{array}{llll}
A_{T L_{R} T L_{A}} & A_{T D_{R} T D_{A}} & A_{B L_{R} B L_{A}} & A_{B D_{R} B D_{A}}
\end{array}\right]\left\{\begin{array}{l}
V_{T L_{R} T L_{A}} \\
V_{T D_{R} T D_{A}} \\
V_{B L_{R} B L_{A}} \\
V_{B D_{R} B D_{A}}
\end{array}\right\}+\left[\begin{array}{c}
B_{T L_{R} T L_{A}} \\
B_{T D_{R} T D_{A}} \\
B_{B L_{R} B L_{A}} \\
B_{B D_{R} B D_{A}}
\end{array}\right] \\
& \{\text { error }\}=\left|\left\{F_{R}\right\}-\left\{F_{A}\right\}\right|
\end{aligned}
$$

The second force calculation method includes the addition of an inverted gain matrix. This procedure is outlined in Barlow, Rae, and Pope, 1999 and is typically used to calibrate force balances. The forces are calculated as before and then each force is divided by the actual known applied force. This gives a gain for each channel for each test point. The gains are averaged over each of the previously described categories to create the average channel gains. A partial example of this gain matrix calculation is shown in Table A.4. One can note that in an ideal situation, the first gain value should be 1 and the rest of the columns 0 . However, it can easily be seen that the onlocation, off-axis sensitivity is high and must be taken into consideration. As mentioned earlier, the off-location 
sensitivities are low in magnitude. Therefore, the low sensitivities are ignored and are set to 0 in the gain matrix in this method.

Table A.4: Example Gain Parameter Calculation (Top Lift Force Applied)

\begin{tabular}{|c|c|c|c|c|}
\hline $\begin{array}{c}\text { Loading } \\
\text { (lbs) }\end{array}$ & $K_{T L_{R} T L_{A}}$ & $K_{T D_{R} T L_{A}}$ & $K_{B L_{R} T L_{A}}$ & $K_{B D_{R} T L_{A}}$ \\
\hline-13.8290 & 1.0430 & 1.2155 & 0.2465 & 0.0821 \\
\hline-9.8362 & 0.9594 & 1.1296 & 0.3553 & 0.1141 \\
\hline-4.0896 & 0.9352 & 1.1621 & 0.6202 & 0.3645 \\
\hline-2.0920 & 0.9042 & 1.4029 & 1.0466 & 0.5429 \\
\hline 0.0000 & - & - & - & - \\
\hline 2.0920 & 0.9246 & -0.1011 & -1.3021 & -0.6181 \\
\hline 4.0896 & 0.9363 & 0.4468 & -0.6487 & -0.2704 \\
\hline 9.8362 & 1.0007 & 0.8572 & -0.3126 & -0.1490 \\
\hline 13.8290 & 0.9879 & 0.9224 & -0.1776 & -0.0595 \\
\hline Average & 0.9614 & 0.8794 & -0.0216 & 0.0008 \\
\hline
\end{tabular}

Once the gain parameters have been calculated, the inverse of the gain matrix is performed as in Equation (A.5) from Barlow, Rae, and Pope, 1999. This procedure creates a more accurate representation of the actual forces whenever the on-axis forces are read from the system. Equation (A.6) and (A.7) shows the expanded form of Equation (A.4) and (A.5).

$$
\begin{aligned}
& \left\{F_{R}\right\}=[K]\left\{F_{A}\right\} \\
& \left\{F_{A}\right\}=[K]^{-1}\left\{F_{R}\right\} \\
& \left\{\begin{array}{l}
F_{T L_{R}} \\
F_{T D_{R}} \\
F_{B L_{R}} \\
F_{B D_{R}}
\end{array}\right\}=\left[\begin{array}{cccc}
K_{T L_{R} T L_{A}} & K_{T L_{R} T D_{A}} & 0 & 0 \\
K_{T D_{R} T L_{A}} & K_{T D_{R} T D_{A}} & 0 & 0 \\
0 & 0 & K_{B L_{R} B L_{A}} & K_{B L_{R} B D_{A}} \\
0 & 0 & K_{B D_{R} B L_{A}} & K_{B D_{R} B D_{A}}
\end{array}\right]\left\{\begin{array}{l}
F_{T L_{A}} \\
F_{T D_{A}} \\
F_{B L_{A}} \\
F_{B D_{A}}
\end{array}\right\} \\
& \left\{\begin{array}{l}
F_{T L_{A}} \\
F_{T D_{A}} \\
F_{B L_{A}} \\
F_{B D_{A}}
\end{array}\right\}=\left[\begin{array}{cccc}
K_{T L_{R} T L_{A}} & K_{T L_{R} T D_{A}} & 0 & 0 \\
K_{T D_{R} T L_{A}} & K_{T D_{R} T D_{A}} & 0 & 0 \\
0 & 0 & K_{B L_{R} B L_{A}} & K_{B L_{R} B D_{A}} \\
0 & 0 & K_{B D_{R} B L_{A}} & K_{B D_{R} B D_{A}}
\end{array}\right]^{-1}\left\{\begin{array}{l}
F_{T L_{R}} \\
F_{T D_{R}} \\
F_{B L_{R}} \\
F_{B D_{R}}
\end{array}\right\}
\end{aligned}
$$


The final method under consideration is the inclusion of all the gain parameters in the gain matrix. This is shown below by the expanded equations (A.8) and (4.1).

$$
\begin{gathered}
\left\{\begin{array}{l}
F_{T L_{R}} \\
F_{T D_{R}} \\
F_{B L_{R}} \\
F_{B D_{R}}
\end{array}\right\}=\left[\begin{array}{llll}
K_{T L_{R} T L_{A}} & K_{T L_{R} T D_{A}} & K_{T L_{R} B L_{A}} & K_{T L_{R} B D_{A}} \\
K_{T D_{R} T L_{A}} & K_{T D_{R} T D_{A}} & K_{T D_{R} B L_{A}} & K_{T D_{R} B D_{A}} \\
K_{B L_{R} T L_{A}} & K_{B L_{R} T D_{A}} & K_{B L_{R} B L_{A}} & K_{B L_{R} B D_{A}} \\
K_{B D_{R} T L_{A}} & K_{B D_{R} T D_{A}} & K_{B D_{R} B L_{A}} & K_{B D_{R} B D_{A}}
\end{array}\right]\left\{\begin{array}{l}
F_{T L_{A}} \\
F_{T D_{A}} \\
F_{B L_{A}} \\
F_{B D_{A}}
\end{array}\right\} \\
\left\{\begin{array}{l}
F_{T L_{A}} \\
F_{T D_{A}} \\
F_{B L_{A}} \\
F_{B D_{A}}
\end{array}\right\}=\left[\begin{array}{llll}
K_{T L_{R} T L_{A}} & K_{T L_{R} T D_{A}} & K_{T L_{R} B L_{A}} & K_{T L_{R} B D_{A}} \\
K_{T D_{R} T L_{A}} & K_{T D_{R} T D_{A}} & K_{T D_{R} B L_{A}} & K_{T D_{R} B D_{A}} \\
K_{B L_{R} T L_{A}} & K_{B L_{R} T D_{A}} & K_{B L_{R} B L_{A}} & K_{B L_{R} B D_{A}} \\
K_{B D_{R} T L_{A}} & K_{B D_{R} T D_{A}} & K_{B D_{R} B L_{A}} & K_{B D_{R} B D_{A}}
\end{array}\right]^{-1}\left\{\begin{array}{l}
F_{T L_{R}} \\
F_{T D_{R}} \\
F_{B L_{R}} \\
F_{B D_{R}}
\end{array}\right\}
\end{gathered}
$$

The calibration methods were then validated by using multiple axis loadings. The same pulley system and calibrated weight set were used to provide loadings in two directions. A similar randomization of the testing schedule was performed. 


\section{A.3 7-Point Load Cell Calibration Curves and Error Calculation}
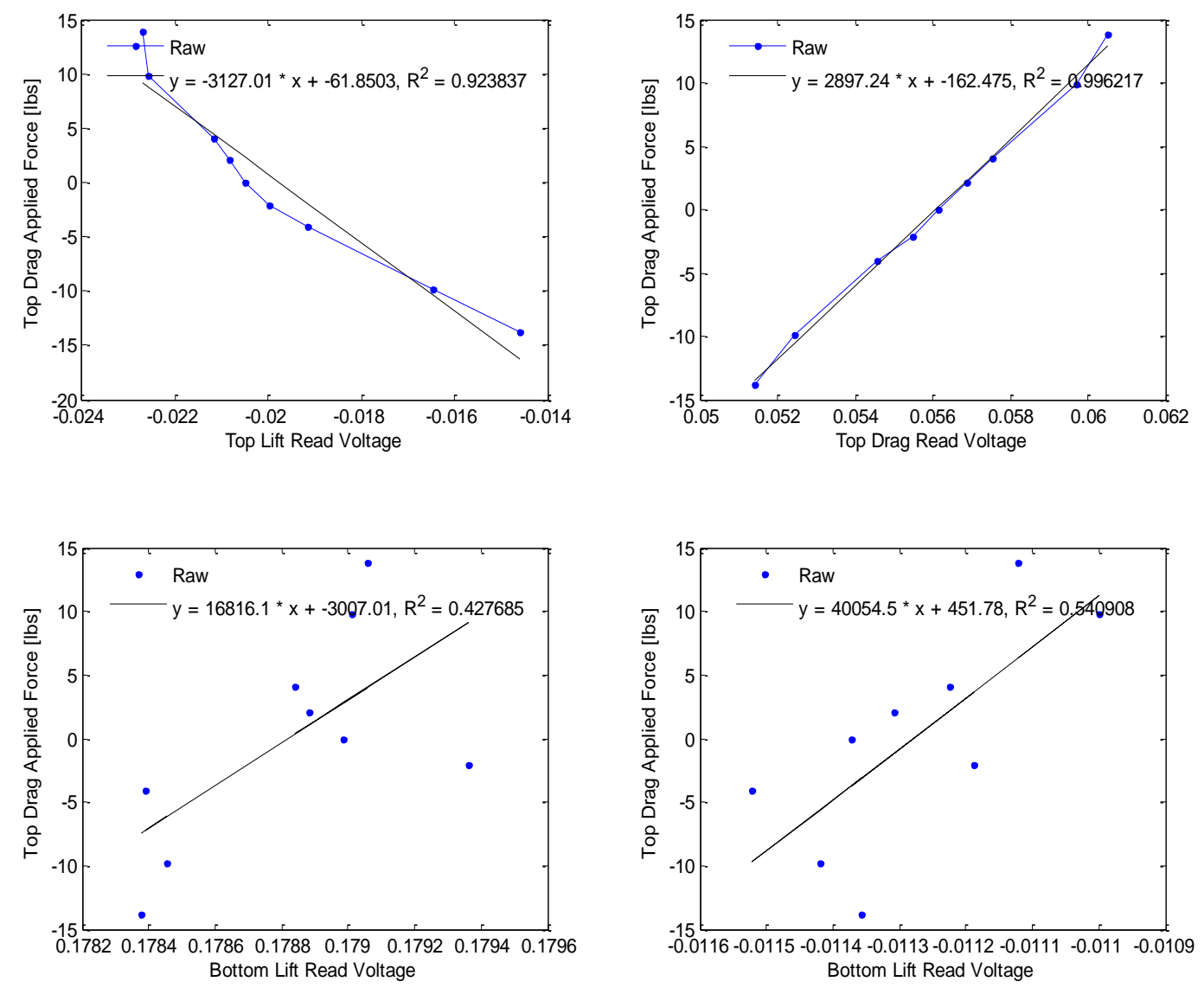

Figure A.3: Top Drag loaded Calibration Curves 

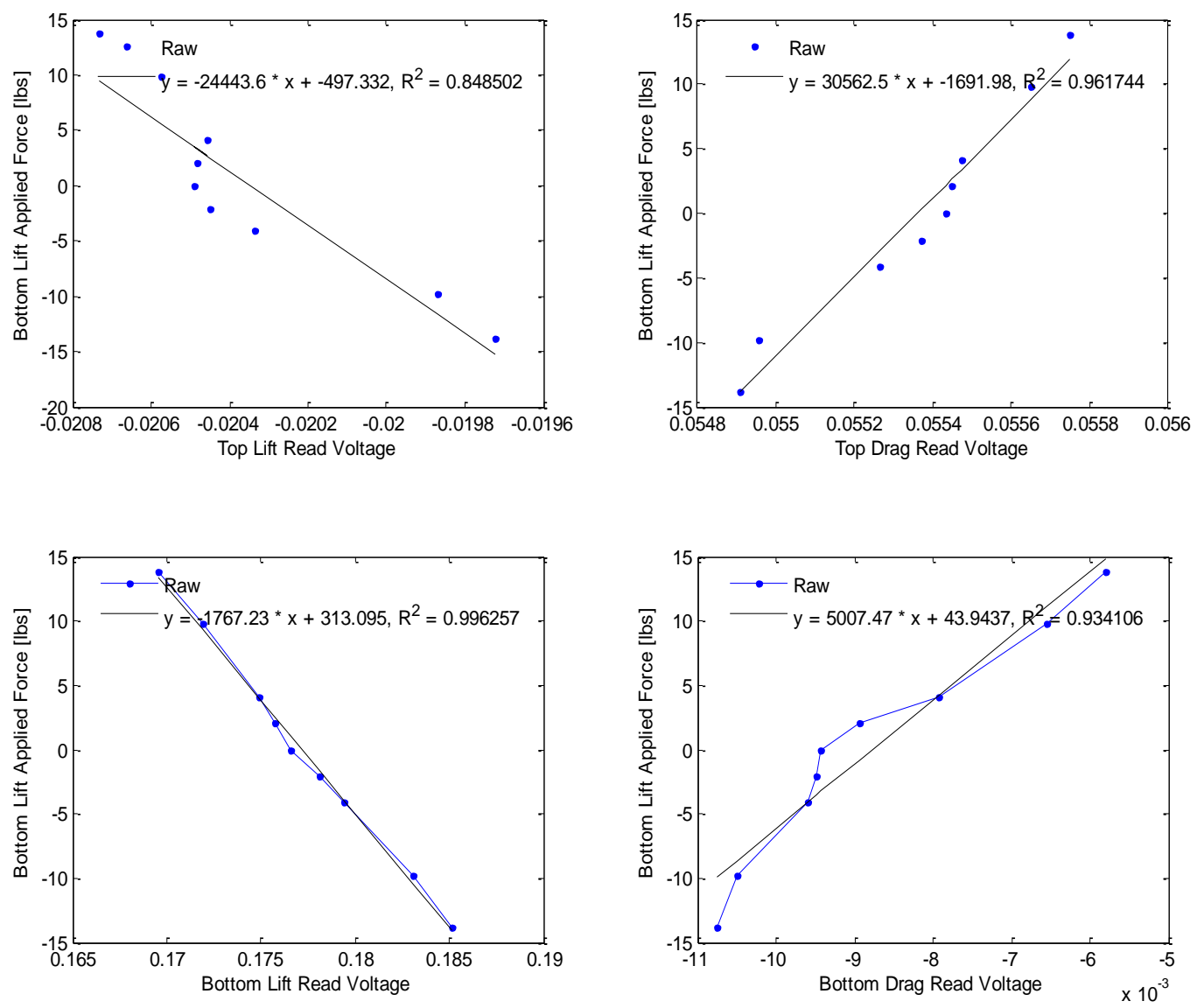

Figure A.4: Bottom Lift Loaded Calibration Curves 

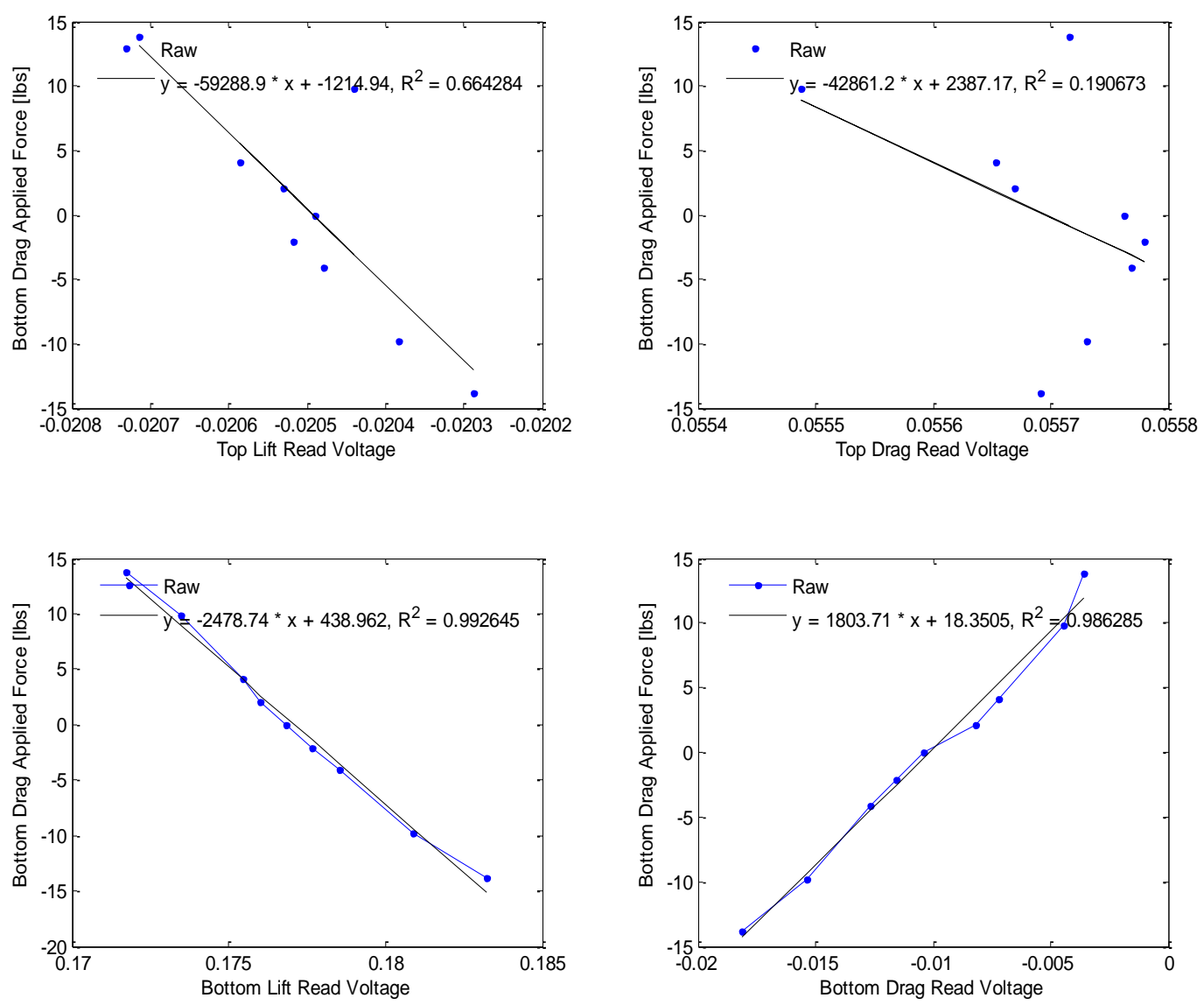

Figure A.5: Bottom Drag Loaded Voltage Readings

Table A.5 shows the forces calculated only using the on-axis regression equations and is color coded for ease of isolation. The pale yellow color designates the on-axis, on-location forces and the pale green color designates the off-axis, on-location forces. The results of the on and off-axis method for force calculation are presented similarly and can be seen in Table A.6. The force calculation method using the on and off-axis on and offlocation method is shown in Table A.7. The error comparison of the different force calculation methods can be seen in Table A.8. 
Table A.5: Forces Calculated Using On-Axis Calibration Curves Only

\begin{tabular}{|c|c|c|c|c|c|c|c|}
\hline \multicolumn{4}{|c|}{ Forces Calculated Using On-Axis Only } & \multicolumn{4}{|c|}{ Loading and Associated Errors } \\
\hline $\mathrm{TL}$ & $\mathrm{TD}$ & $\mathrm{BL}$ & $\mathrm{BD}$ & Loading & Loading Err & Max Err & Avg Err \\
\hline-14.424 & -16.809 & -3.409 & -1.135 & -13.829 & 0.595 & 16.809 & 5.487 \\
\hline-9.437 & -11.111 & -3.494 & -1.122 & -9.836 & 0.399 & 11.111 & 4.031 \\
\hline-3.825 & -4.753 & -2.536 & -1.491 & -4.090 & 0.265 & 4.753 & 2.261 \\
\hline-1.892 & -2.935 & -2.189 & -1.136 & -2.092 & 0.200 & 2.935 & 1.615 \\
\hline 0.309 & -1.710 & -2.897 & -1.418 & 0.000 & 0.309 & 2.897 & 1.584 \\
\hline 1.934 & -0.212 & -2.724 & -1.293 & 2.092 & 0.158 & 2.724 & 1.097 \\
\hline 3.829 & 1.827 & -2.653 & -1.106 & 4.090 & 0.260 & 2.653 & 1.462 \\
\hline 9.843 & 8.432 & -3.075 & -1.466 & 9.836 & 0.007 & 8.432 & 3.245 \\
\hline 13.661 & 12.755 & -2.456 & -0.823 & 13.829 & 0.168 & 12.755 & 4.051 \\
\hline-7.790 & -13.515 & -2.136 & -2.133 & -13.829 & 0.314 & 7.790 & 3.093 \\
\hline-5.024 & -10.517 & -2.277 & -2.248 & -9.836 & 0.681 & 5.024 & 2.557 \\
\hline-1.114 & -4.420 & -2.160 & -2.431 & -4.090 & 0.331 & 2.431 & 1.509 \\
\hline 0.102 & -1.668 & -3.881 & -1.829 & -2.092 & 0.424 & 3.881 & 1.559 \\
\hline 0.902 & 0.203 & -3.214 & -2.162 & 0.000 & 0.203 & 3.214 & 1.620 \\
\hline 1.395 & 2.272 & -3.033 & -2.045 & 2.092 & 0.180 & 3.033 & 1.663 \\
\hline 1.890 & 4.211 & -2.957 & -1.897 & 4.090 & 0.122 & 2.957 & 1.716 \\
\hline 3.961 & 10.550 & -3.258 & -1.489 & 9.836 & 0.713 & 3.961 & 2.355 \\
\hline 4.136 & 12.885 & -3.340 & -1.710 & 13.829 & 0.944 & 4.136 & 2.532 \\
\hline-0.252 & -3.393 & -14.058 & -1.050 & -13.829 & 0.229 & 3.393 & 1.231 \\
\hline-0.038 & -3.250 & -10.467 & -0.595 & -9.836 & 0.630 & 3.250 & 1.128 \\
\hline 0.644 & -2.359 & -4.013 & 1.051 & -4.090 & 0.077 & 2.359 & 1.033 \\
\hline 0.812 & -2.050 & -1.677 & 1.245 & -2.092 & 0.415 & 2.050 & 1.130 \\
\hline 0.872 & -1.877 & 1.035 & 1.355 & 0.000 & 1.035 & 1.877 & 1.285 \\
\hline 0.863 & -1.829 & 2.479 & 2.224 & 2.092 & 0.387 & 2.224 & 1.326 \\
\hline 0.826 & -1.757 & 3.980 & 4.040 & 4.090 & 0.110 & 4.040 & 1.683 \\
\hline 0.995 & -1.248 & 9.298 & 6.545 & 9.836 & 0.539 & 6.545 & 2.331 \\
\hline 1.231 & -0.953 & 13.426 & 7.881 & 13.829 & 0.403 & 7.881 & 2.617 \\
\hline 0.576 & -1.122 & -10.691 & -14.312 & -13.829 & 0.483 & 10.691 & 3.218 \\
\hline 0.716 & -1.012 & -6.559 & -9.399 & -9.836 & 0.437 & 6.559 & 2.181 \\
\hline 0.856 & -0.901 & -2.428 & -4.486 & -4.090 & 0.397 & 2.428 & 1.145 \\
\hline 0.912 & -0.869 & -0.855 & -2.516 & -2.092 & 0.424 & 0.912 & 0.765 \\
\hline 0.871 & -0.917 & 0.564 & -0.419 & 0.000 & 0.419 & 0.917 & 0.693 \\
\hline 0.932 & -1.187 & 2.015 & 3.552 & 2.092 & 1.460 & 2.015 & 1.399 \\
\hline 1.014 & -1.233 & 3.063 & 5.325 & 4.090 & 1.235 & 3.063 & 1.636 \\
\hline 0.799 & -1.714 & 6.515 & 10.333 & 9.836 & 0.497 & 6.515 & 2.382 \\
\hline 1.203 & -1.052 & 9.588 & 11.923 & 13.829 & 1.906 & 9.588 & 3.437 \\
\hline
\end{tabular}


Table A.6: Forces Calculated Using On and Off Axis Calibration Curves Only

\begin{tabular}{|c|c|c|c|c|c|c|c|}
\hline \multicolumn{4}{|c|}{$\begin{array}{c}\text { Forces Calculated Using On and Off } \\
\text { Axis Curves }\end{array}$} & \multicolumn{4}{|c|}{ Loading and Associated Errors } \\
\hline $\mathrm{TL}$ & TD & BL & BD & Loading & Loading Err & Max Err & Avg Err \\
\hline-12.739 & -5.536 & -3.409 & -0.038 & -13.829 & 1.090 & 5.536 & 2.518 \\
\hline-8.264 & -3.796 & -3.526 & 0.006 & -9.836 & 1.573 & 3.796 & 2.225 \\
\hline-3.193 & -1.923 & -2.053 & -0.724 & -4.090 & 0.897 & 2.053 & 1.399 \\
\hline-1.213 & -1.852 & -1.885 & -0.462 & -2.092 & 0.879 & 1.885 & 1.270 \\
\hline 1.571 & -3.075 & -2.558 & -0.521 & 0.000 & 1.571 & 3.075 & 1.931 \\
\hline 3.254 & -3.060 & -2.435 & -0.446 & 2.092 & 1.162 & 3.060 & 1.776 \\
\hline 5.034 & -2.594 & -2.487 & -0.269 & 4.090 & 0.944 & 2.594 & 1.573 \\
\hline 10.598 & -0.908 & -2.744 & -0.510 & 9.836 & 0.762 & 2.744 & 1.231 \\
\hline 14.049 & 0.363 & -2.453 & -0.033 & 13.829 & 0.220 & 2.453 & 0.767 \\
\hline-4.097 & -9.833 & -1.072 & -1.555 & -13.829 & 3.996 & 4.097 & 2.680 \\
\hline-1.515 & -9.118 & -1.163 & -1.629 & -9.836 & 0.718 & 1.629 & 1.256 \\
\hline 0.974 & -5.242 & -0.878 & -1.867 & -4.090 & 1.153 & 1.867 & 1.218 \\
\hline 1.210 & -2.717 & -3.479 & -0.622 & -2.092 & 0.625 & 3.479 & 1.484 \\
\hline 1.329 & -0.962 & -2.397 & -1.211 & 0.000 & 0.962 & 2.397 & 1.475 \\
\hline 0.828 & 1.531 & -2.259 & -1.148 & 2.092 & 0.561 & 2.259 & 1.199 \\
\hline 0.409 & 3.824 & -2.275 & -1.015 & 4.090 & 0.266 & 2.275 & 0.991 \\
\hline-0.221 & 10.670 & -2.956 & -0.472 & 9.836 & 0.834 & 2.956 & 1.120 \\
\hline-1.403 & 14.025 & -2.894 & -0.680 & 13.829 & 0.196 & 2.894 & 1.293 \\
\hline 1.720 & -4.875 & -16.778 & 3.751 & -13.829 & 2.949 & 4.875 & 3.324 \\
\hline 1.976 & -4.958 & -12.631 & 2.994 & -9.836 & 2.795 & 4.958 & 3.181 \\
\hline 2.517 & -4.548 & -5.800 & 2.525 & -4.090 & 1.711 & 4.548 & 2.825 \\
\hline 2.594 & -4.308 & -3.027 & 1.923 & -2.092 & 0.935 & 4.308 & 2.440 \\
\hline 2.583 & -4.127 & 0.279 & 1.099 & 0.000 & 0.279 & 4.127 & 2.022 \\
\hline 2.540 & -4.041 & 1.432 & 1.533 & 2.092 & 0.659 & 4.041 & 2.193 \\
\hline 2.435 & -3.878 & 1.948 & 2.966 & 4.090 & 2.141 & 3.878 & 2.855 \\
\hline 2.387 & -3.330 & 6.717 & 3.816 & 9.836 & 3.119 & 3.816 & 3.163 \\
\hline 2.583 & -3.209 & 10.875 & 3.821 & 13.829 & 2.954 & 3.821 & 3.142 \\
\hline 1.633 & -2.544 & -2.644 & -11.693 & -13.829 & 2.136 & 2.644 & 2.239 \\
\hline 1.789 & -2.572 & -1.159 & -7.839 & -9.836 & 1.997 & 2.572 & 1.879 \\
\hline 1.946 & -2.599 & 0.325 & -3.986 & -4.090 & 0.104 & 2.599 & 1.243 \\
\hline 2.017 & -2.630 & 0.815 & -2.411 & -2.092 & 0.319 & 2.630 & 1.445 \\
\hline 1.981 & -2.645 & 1.019 & -0.647 & 0.000 & 0.647 & 2.645 & 1.573 \\
\hline 2.248 & -3.148 & -0.141 & 3.124 & 2.092 & 1.032 & 3.148 & 1.642 \\
\hline 2.409 & -3.334 & -0.159 & 4.668 & 4.090 & 0.578 & 3.334 & 1.620 \\
\hline 2.365 & -3.774 & 0.405 & 8.860 & 9.836 & 0.976 & 3.774 & 1.880 \\
\hline 2.160 & -2.823 & 3.095 & 9.540 & 13.829 & 4.289 & 4.289 & 3.092 \\
\hline
\end{tabular}


Table A.7: Forces Calculated Using All Calibration Curves (Matrix)

\begin{tabular}{|c|c|c|c|c|c|c|c|}
\hline \multicolumn{4}{|c|}{ Forces Calculated Using All Axes } & \multicolumn{4}{|c|}{ Loading and Associated Errors } \\
\hline $\mathrm{TL}$ & $\mathrm{TD}$ & $\mathrm{BL}$ & $\mathrm{BD}$ & Loading & Loading Err & Max Err & Avg Err \\
\hline-12.713 & -5.292 & -3.810 & 0.164 & -13.829 & 1.116 & 5.292 & 2.596 \\
\hline-8.239 & -3.541 & -3.788 & 0.141 & -9.836 & 1.598 & 3.788 & 2.267 \\
\hline-3.146 & -1.830 & -2.158 & -0.664 & -4.090 & 0.944 & 2.158 & 1.399 \\
\hline-1.179 & -1.751 & -1.935 & -0.420 & -2.092 & 0.913 & 1.935 & 1.255 \\
\hline 1.613 & -2.929 & -2.543 & -0.481 & 0.000 & 1.613 & 2.929 & 1.891 \\
\hline 3.291 & -2.918 & -2.373 & -0.421 & 2.092 & 1.199 & 2.918 & 1.728 \\
\hline 5.063 & -2.434 & -2.371 & -0.267 & 4.090 & 0.974 & 2.434 & 1.512 \\
\hline 10.640 & -0.748 & -2.460 & -0.584 & 9.836 & 0.804 & 2.460 & 1.149 \\
\hline 14.068 & 0.538 & -2.062 & -0.157 & 13.829 & 0.239 & 2.062 & 0.749 \\
\hline-4.021 & -9.874 & -1.273 & -1.351 & -13.829 & 3.955 & 4.021 & 2.650 \\
\hline-1.434 & -9.157 & -1.287 & -1.459 & -9.836 & 0.679 & 1.459 & 1.215 \\
\hline 1.062 & -5.320 & -0.899 & -1.785 & -4.090 & 1.231 & 1.785 & 1.244 \\
\hline 1.263 & -2.512 & -3.470 & -0.585 & -2.092 & 0.420 & 3.470 & 1.435 \\
\hline 1.399 & -0.881 & -2.369 & -1.206 & 0.000 & 0.881 & 2.369 & 1.464 \\
\hline 0.894 & 1.608 & -2.222 & -1.181 & 2.092 & 0.484 & 2.222 & 1.196 \\
\hline 0.471 & 3.911 & -2.229 & -1.084 & 4.090 & 0.178 & 2.229 & 0.990 \\
\hline-0.179 & 10.848 & -2.865 & -0.654 & 9.836 & 1.012 & 2.865 & 1.177 \\
\hline-1.352 & 14.182 & -2.806 & -0.910 & 13.829 & 0.353 & 2.806 & 1.355 \\
\hline 1.675 & -3.378 & -16.774 & 3.821 & -13.829 & 2.945 & 3.821 & 2.955 \\
\hline 1.935 & -3.818 & -12.621 & 3.063 & -9.836 & 2.785 & 3.818 & 2.900 \\
\hline 2.448 & -3.937 & -5.772 & 2.582 & -4.090 & 1.682 & 3.937 & 2.662 \\
\hline 2.532 & -3.943 & -2.995 & 1.975 & -2.092 & 0.903 & 3.943 & 2.338 \\
\hline 2.533 & -4.064 & 0.313 & 1.148 & 0.000 & 0.313 & 4.064 & 2.015 \\
\hline 2.462 & -4.028 & 1.466 & 1.582 & 2.092 & 0.626 & 4.028 & 2.174 \\
\hline 2.290 & -3.794 & 1.981 & 3.013 & 4.090 & 2.109 & 3.794 & 2.802 \\
\hline 2.171 & -3.527 & 6.753 & 3.853 & 9.836 & 3.083 & 3.853 & 3.159 \\
\hline 2.337 & -3.706 & 10.917 & 3.855 & 13.829 & 2.912 & 3.855 & 3.202 \\
\hline 2.167 & -3.239 & -2.621 & -11.663 & -13.829 & 2.166 & 3.239 & 2.548 \\
\hline 2.143 & -3.082 & -1.133 & -7.810 & -9.836 & 2.026 & 3.082 & 2.096 \\
\hline 2.119 & -2.924 & 0.355 & -3.958 & -4.090 & 0.132 & 2.924 & 1.383 \\
\hline 2.117 & -2.871 & 0.847 & -2.383 & -2.092 & 0.291 & 2.871 & 1.532 \\
\hline 2.002 & -2.768 & 1.050 & -0.618 & 0.000 & 0.618 & 2.768 & 1.609 \\
\hline 2.111 & -2.901 & -0.108 & 3.159 & 2.092 & 1.067 & 2.901 & 1.547 \\
\hline 2.205 & -2.969 & -0.122 & 4.705 & 4.090 & 0.615 & 2.969 & 1.478 \\
\hline 1.972 & -3.132 & 0.436 & 8.905 & 9.836 & 0.931 & 3.132 & 1.618 \\
\hline 2.160 & -2.823 & 3.095 & 9.540 & 13.829 & 4.289 & 4.289 & 3.092 \\
\hline
\end{tabular}


Table A.8: Error Comparison for Calibration Methods Using Calibration Data

\begin{tabular}{|c|c|c|c|c|c|c|}
\hline \multirow{2}{*}{ Calibration Curves Used } & \multicolumn{2}{|c|}{ On Axis Only } & \multicolumn{2}{c|}{ On and Off Axis } & \multicolumn{2}{c|}{ All Axes } \\
\hline Value & Max Error & Avg Error & Max Error & Avg Error & Max Error & Avg Error \\
\hline Error & 16.809 & 2.057 & 5.536 & 1.928 & 5.292 & 1.899 \\
\hline \% Error Full Scale Test & 60.775 & 7.438 & 20.016 & 6.970 & 19.135 & 6.868 \\
\hline \% Error Full Scale LC & 33.618 & 4.114 & 11.072 & 3.856 & 10.585 & 3.799 \\
\hline
\end{tabular}

\section{A.4 Load Cell Multiple-Loading Validation}

Table A.9 shows the forces calculated only using the on-axis regression equations. The results of the on and off-axis method for force calculation are shown in Table A.10. The force calculation method using the on and offaxis on and off-location method are presented in Table A.11. The error of the different force calculation methods is compared in Table A.12.

Table A.9: Multiple-Loading Validation Using On-Axis Calibration Curves Only

\begin{tabular}{|c|c|c|c|c|c|c|c|c|c|c|c|c|c|c|}
\hline & \multicolumn{4}{|c|}{ On Axis Calc } & \multicolumn{4}{|c|}{ Loading } & \multicolumn{4}{|c|}{ Loading Error } & \multirow{2}{*}{$\begin{array}{c}\text { Maximum } \\
\text { Error }\end{array}$} & \multirow{2}{*}{$\begin{array}{c}\text { Average } \\
\text { Error }\end{array}$} \\
\hline & $\mathrm{TL}$ & $\mathrm{TD}$ & $\mathrm{BL}$ & BD & TL & $\mathrm{TD}$ & $\mathrm{BL}$ & BD & $\mathrm{TL}$ & TD & $\mathrm{BL}$ & BD & & \\
\hline NBLM PBDL & 0.00 & -2.72 & -1.39 & 2.81 & 0.00 & 0.00 & -4.09 & 2.09 & 0.00 & 2.72 & -2.70 & -0.72 & 2.72 & 1.54 \\
\hline NTLM_PTDL & -3.25 & -3.25 & -2.83 & -1.03 & -4.09 & 2.09 & 0.00 & 0.00 & -0.84 & 5.34 & 2.83 & 1.03 & 34 & 2.51 \\
\hline PBLH_PBDL & 1.10 & -0.68 & 9.02 & 7.93 & 0.00 & 0.00 & 9.84 & 2.09 & -1.10 & 0.68 & 0.82 & -5.84 & .84 & 2.11 \\
\hline PBLL_PBDM & 0.87 & -1.39 & 4.62 & 6.14 & 0.00 & 0.00 & 2.09 & 4.09 & -0.87 & 1.39 & -2.53 & -2.05 & 2.53 & 1.71 \\
\hline PTL0_PTD0 & 0.22 & -1.55 & -1.90 & -2.11 & 0.00 & 0.00 & 0.00 & 0.00 & -0.22 & 1.55 & 1.90 & 2.11 & 2.11 & 1.44 \\
\hline PTLH_PTDL & 10.16 & 10.48 & -2.99 & -1.00 & 9.84 & 2.09 & 0.00 & 0.00 & -0.32 & -8.39 & 2.99 & 1.00 & 8.39 & 3.17 \\
\hline PTLXH_PTDL & 14.02 & 15.45 & -1.54 & -1.53 & 13.83 & 2.09 & 0.00 & 0.00 & -0.19 & -13.36 & 1.54 & 1.53 & 13.36 & 4.16 \\
\hline
\end{tabular}

Table A.10: Multiple-Loading Validation Using On and Off Axis Calibration Curves

\begin{tabular}{|c|c|c|c|c|c|c|c|c|c|c|c|c|c|c|}
\hline & \multicolumn{4}{|c|}{ On and Off Axis Calc } & \multicolumn{4}{|c|}{ Loading } & \multicolumn{4}{|c|}{ Loading Error } & \multirow{2}{*}{$\begin{array}{c}\text { Maximum } \\
\text { Error }\end{array}$} & \multirow{2}{*}{$\begin{array}{c}\text { Average } \\
\text { Error }\end{array}$} \\
\hline & TL & TD & $\mathrm{BL}$ & $\mathrm{BD}$ & TL & TD & $\mathrm{BL}$ & $\mathrm{BD}$ & $\mathrm{TL}$ & TD & $\mathrm{BL}$ & $\mathrm{BD}$ & & \\
\hline NBLM PBDL & 1.71 & -4.20 & -3.83 & 3.51 & 0.00 & 0.00 & -4.09 & 2.09 & -1.71 & 4.20 & -0.26 & -1.41 & 4.20 & 1.89 \\
\hline NTLM_PTDL & -3.21 & -0.42 & -2.76 & -0.13 & -4.09 & 2.09 & 0.00 & 0.00 & -0.88 & 2.51 & 2.76 & 0. & 76 & 1.57 \\
\hline PBLH_PBDL & 2.20 & -2.61 & 5.33 & 5.40 & 0.00 & 0.00 & 9.84 & 2.09 & -2.20 & 2.61 & 4.50 & -3.31 & 4.50 & 3.16 \\
\hline PBLL_PBDM & 2.28 & -3.37 & 1.18 & 5.01 & 0.00 & 0.00 & 2.09 & 4.09 & -2.28 & 3.37 & 0.91 & -0.92 & 3.37 & 1.87 \\
\hline PTL0_PTD0 & 1.33 & -2.71 & -0.79 & -1.61 & 0.00 & 0.00 & 0.00 & 0.00 & -1.33 & 2.71 & 0.79 & 1.61 & 2.71 & 1.61 \\
\hline PTLH_PTDL & 9.82 & 1.80 & -2.99 & -0.04 & 9.84 & 2.09 & 0.00 & 0.00 & 0.01 & 0.29 & 2.99 & 0.04 & 2.99 & 0.83 \\
\hline PTLXH_PTDL & 12.94 & 4.01 & -0.78 & -1.11 & 13.83 & 2.09 & 0.00 & 0.00 & 0.89 & -1.92 & 0.78 & 1.11 & 1.92 & 1.17 \\
\hline
\end{tabular}


Table A.11: Multiple-Loading Validation Using All Axis Calibration

\begin{tabular}{|c|c|c|c|c|c|c|c|c|c|c|c|c|c|c|}
\hline & \multicolumn{4}{|c|}{ On Axis Calc } & \multicolumn{4}{|c|}{ Loading } & \multicolumn{4}{|c|}{ Loading Error } & \multirow{2}{*}{$\begin{array}{c}\text { Maximum } \\
\text { Error }\end{array}$} & \multirow{2}{*}{$\begin{array}{c}\text { Average } \\
\text { Error }\end{array}$} \\
\hline & $\mathrm{TL}$ & TD & $\mathrm{BL}$ & $\mathrm{BD}$ & $\mathrm{TL}$ & $\mathrm{TD}$ & $\mathrm{BL}$ & $\mathrm{BD}$ & TL & $\mathrm{TD}$ & $\mathrm{BL}$ & $\mathrm{BD}$ & & \\
\hline NBLM_PBDL & 0.00 & -2.72 & -1.39 & 2.81 & 0.00 & 0.00 & -4.09 & 2.09 & 0.00 & 2.72 & -2.70 & -0.72 & 2.72 & 1.54 \\
\hline NTLM_PTDL & -3.25 & -3.25 & -2.83 & -1.03 & -4.09 & 2.09 & 0.00 & 0.00 & -0.84 & 5.34 & 2.83 & 1.03 & 5.34 & 2.51 \\
\hline PBLH_PBDL & 1.10 & -0.68 & 9.02 & 7.93 & 0.00 & 0.00 & 9.84 & 2.09 & -1.10 & 0.68 & 0.82 & -5.84 & 5.84 & 2.11 \\
\hline PBLL_PBDM & 0.87 & -1.39 & 4.62 & 6.14 & 0.00 & 0.00 & 2.09 & 4.09 & -0.87 & 1.39 & -2.53 & -2.05 & 2.53 & 1.71 \\
\hline PTL0_PTD0 & 0.22 & -1.55 & -1.90 & -2.11 & 0.00 & 0.00 & 0.00 & 0.00 & -0.22 & 1.55 & 1.90 & 2.11 & 2.11 & 1.44 \\
\hline PTLH_PTDL & 10.16 & 10.48 & -2.99 & -1.00 & 9.84 & 2.09 & 0.00 & 0.00 & -0.32 & -8.39 & 2.99 & 1.00 & 8.39 & 3.17 \\
\hline PTLXH_PTDL & 14.02 & 15.45 & -1.54 & -1.53 & 13.83 & 2.09 & 0.00 & 0.00 & -0.19 & -13.36 & 1.54 & 1.53 & 13.36 & 4.16 \\
\hline
\end{tabular}

Table A.12: Error Comparison for Calibration Methods Using Multiple-Loading Validation Data

\begin{tabular}{|l|c|c|c|c|c|c|}
\hline \multirow{2}{*}{ Calibration Curves Used } & \multicolumn{2}{|c|}{ On Axis Only } & \multicolumn{2}{c|}{ On and Off Axis } & \multicolumn{2}{c|}{ All Axes } \\
\hline \multicolumn{1}{|c|}{ Value } & Max Error & Avg Error & Max Error & Avg Error & Max Error & Avg Error \\
\hline Error & 13.36 & 2.38 & 4.50 & 1.73 & 4.47 & 1.65 \\
\hline$\%$ Error Full Scale Test & 48.30 & 8.60 & 16.28 & 6.26 & 16.15 & 5.98 \\
\hline \% Error Full Scale LC & 26.72 & 4.76 & 9.01 & 3.46 & 8.93 & 3.31 \\
\hline
\end{tabular}




\section{Appendix B - Detailed Results for Lift and Drag Coefficient}

\section{B.1 Lift Coefficient Summary}

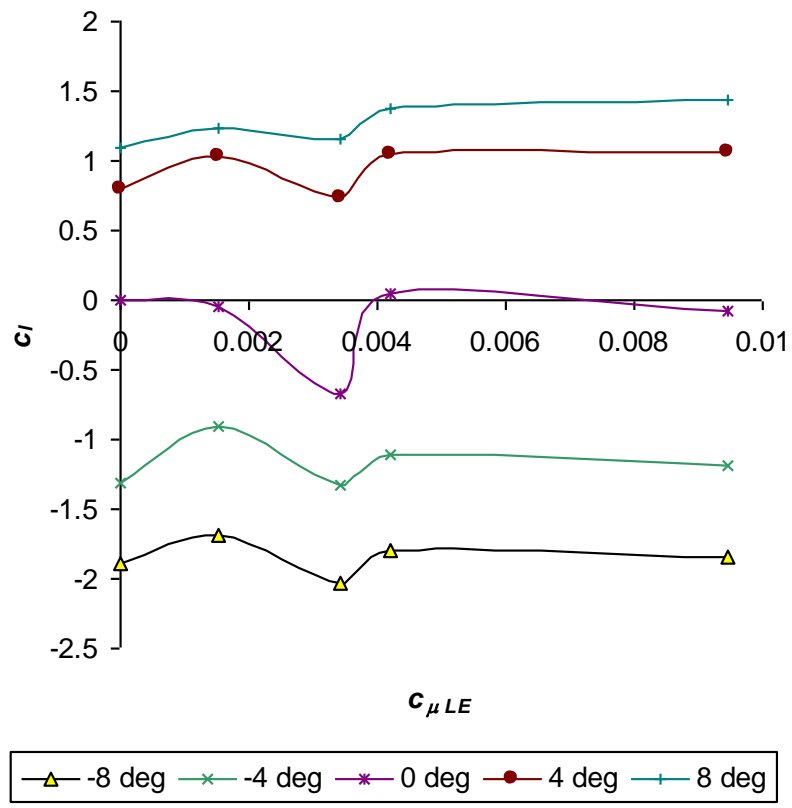

Figure B.6: Lift Coefficient vs. Leading Edge Blowing Coefficient vs. Angle of Attack

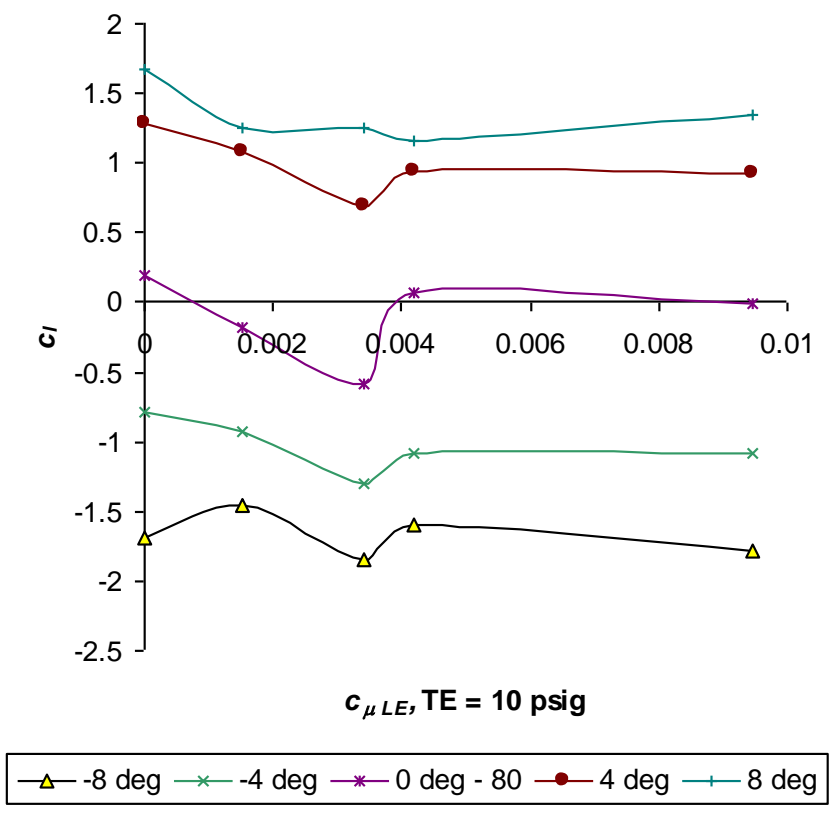

Figure B.7: Lift Coefficient vs. Leading Edge Blowing Coefficient vs. Angle of Attack, Trailing Edge Blowing $=10 \mathrm{psig}$ 


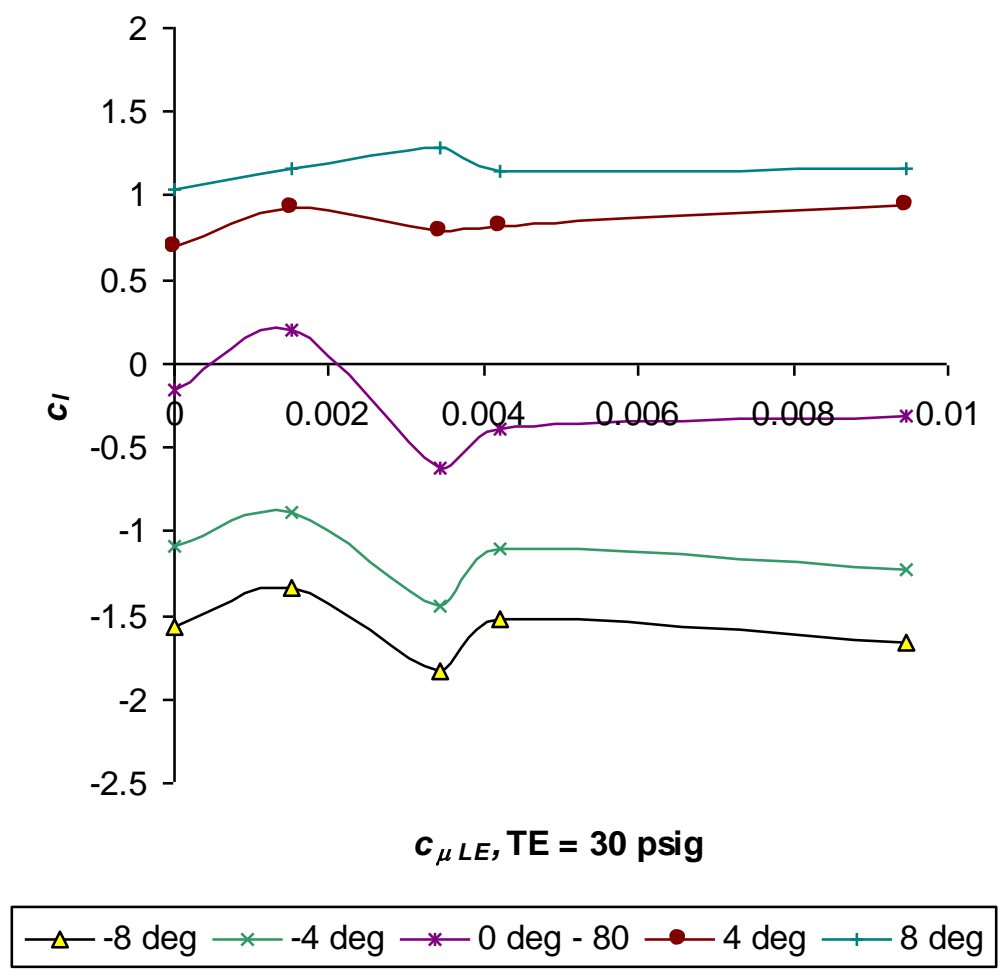

Figure B.8: Lift Coefficient vs. Leading Edge Blowing Coefficient vs. Angle of Attack, Trailing Edge Blowing $=30 \mathrm{psig}$

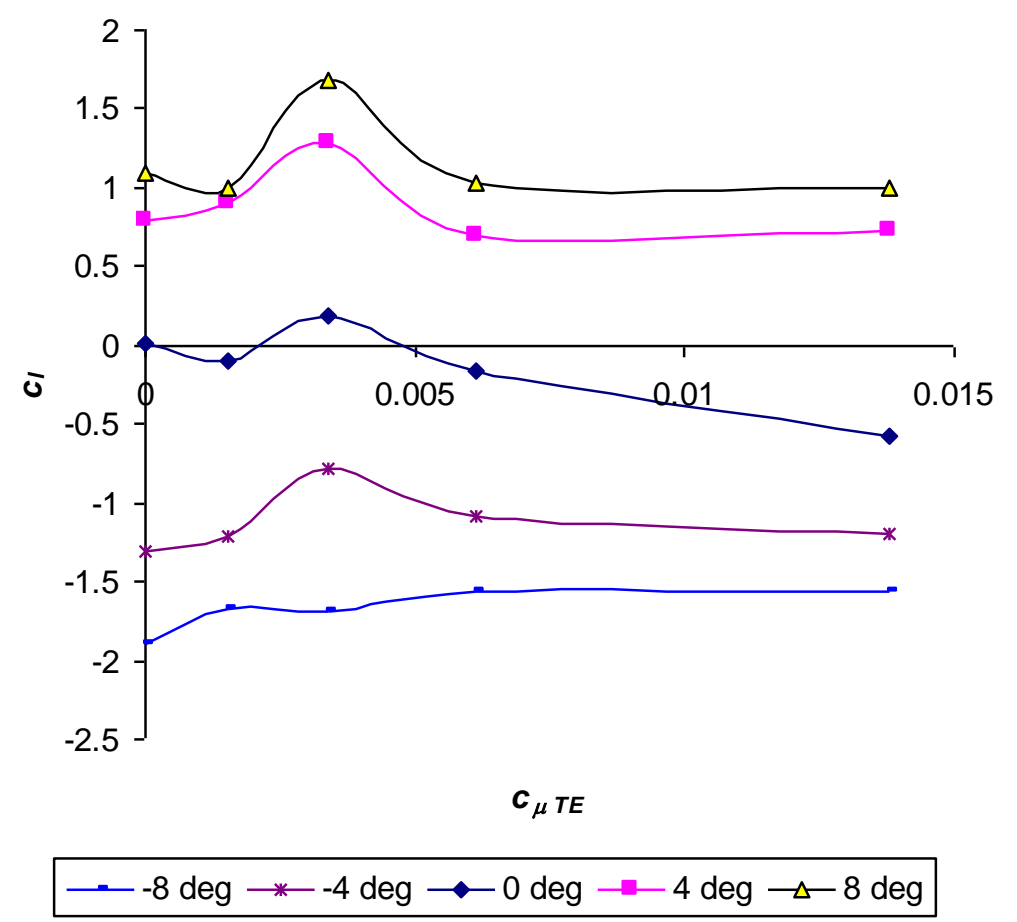

Figure B.9: Lift Coefficient vs. Trailing Edge Blowing Coefficient vs. Angle of Attack 


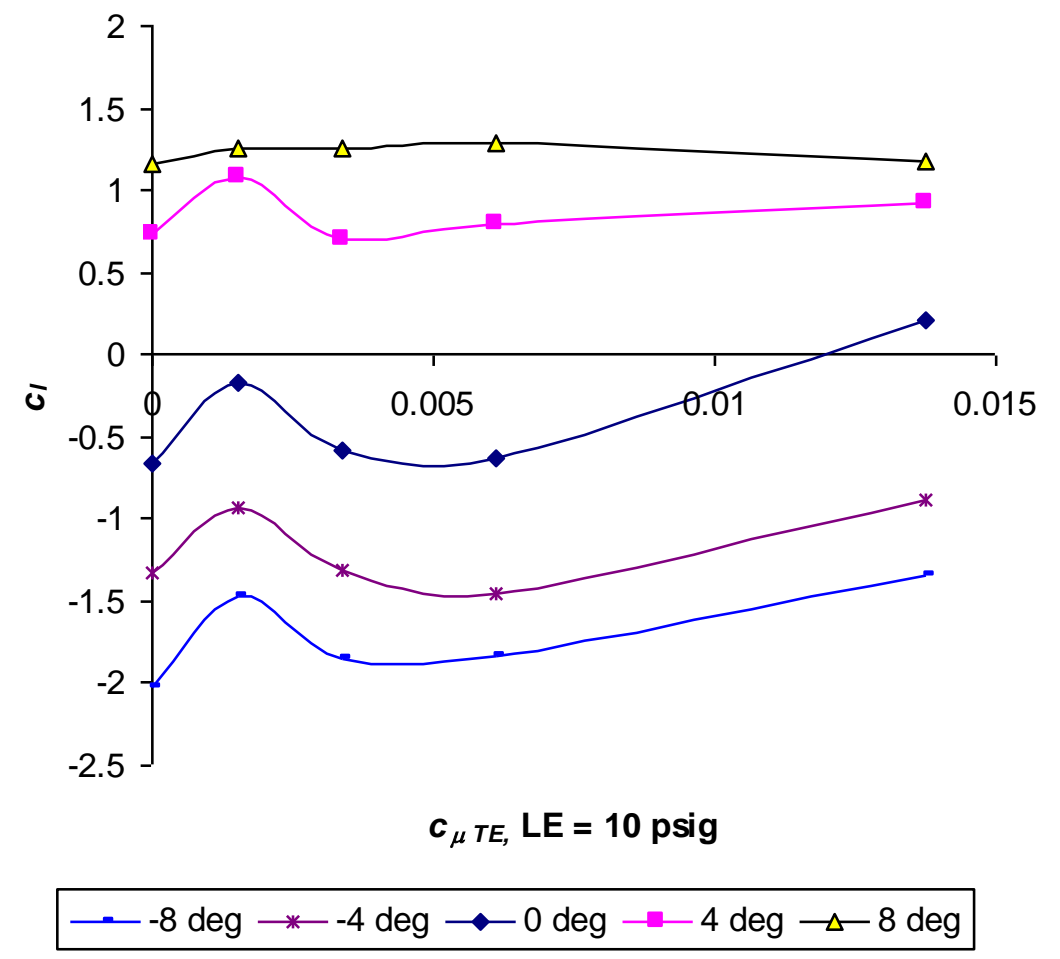

Figure B.10: Lift Coefficient vs. Trailing Edge Blowing Coefficient vs. Angle of Attack, Leading Edge Blowing $=10 \mathrm{psig}$

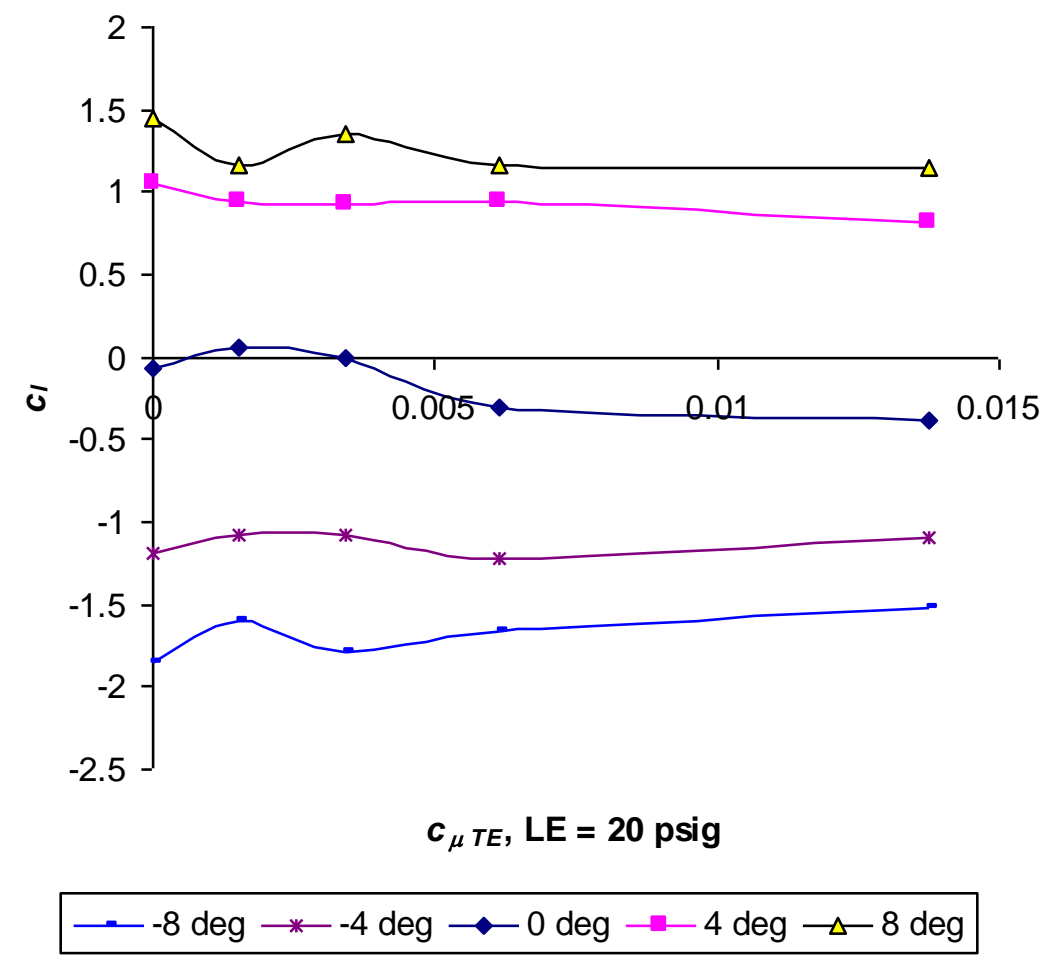

Figure B.11: Lift Coefficient vs. Trailing Edge Blowing Coefficient vs. Angle of Attack, Leading Edge Blowing $=20$ psig 


\section{B.2 Lift Coefficient - Trailing Edge Varying - Average Tare Method}

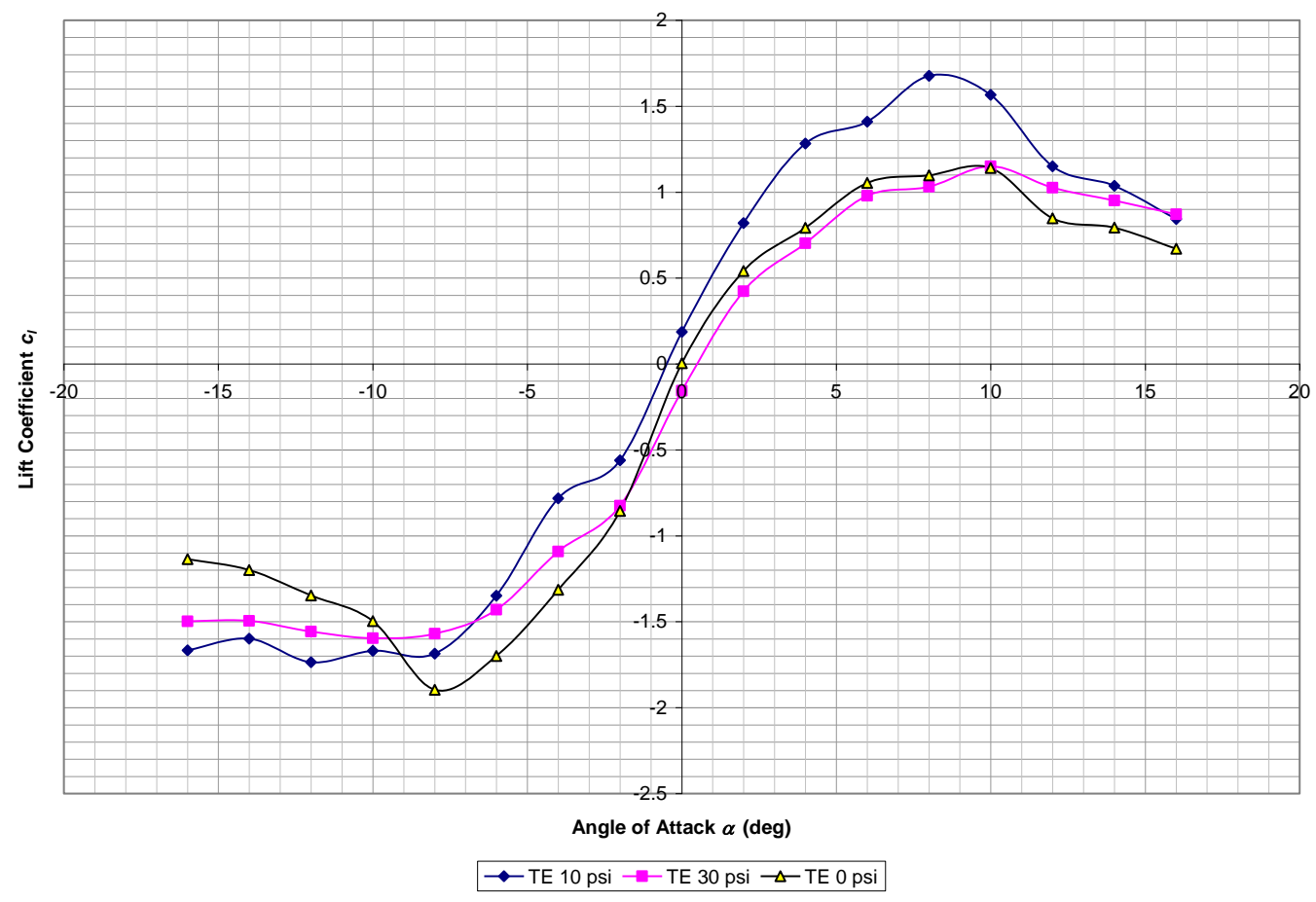

Figure B.12: Tunnel Velocity $=80 \mathrm{fps}$, Trailing Edge Blowing Only, Lift Coefficient vs. Angle of Attack (average tare)

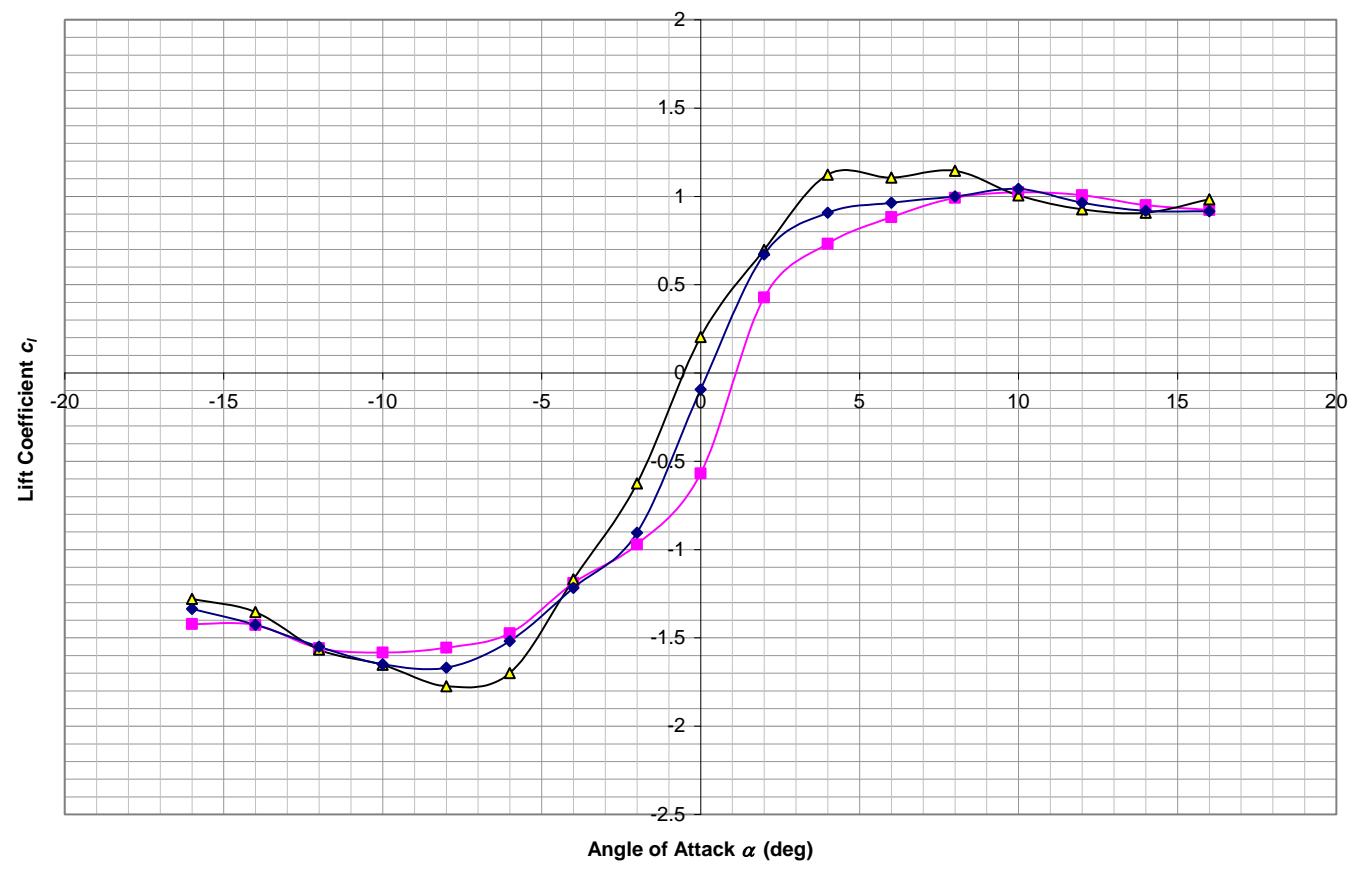

$\rightarrow-$ TE 30 psi $\triangle-$ TE 0 psi $\rightarrow-$ TE 10 psi

Figure B.13: Tunnel Velocity $=120 \mathrm{fps}$, Trailing Edge Blowing Only, Lift Coefficient vs. Angle of Attack (average tare) 


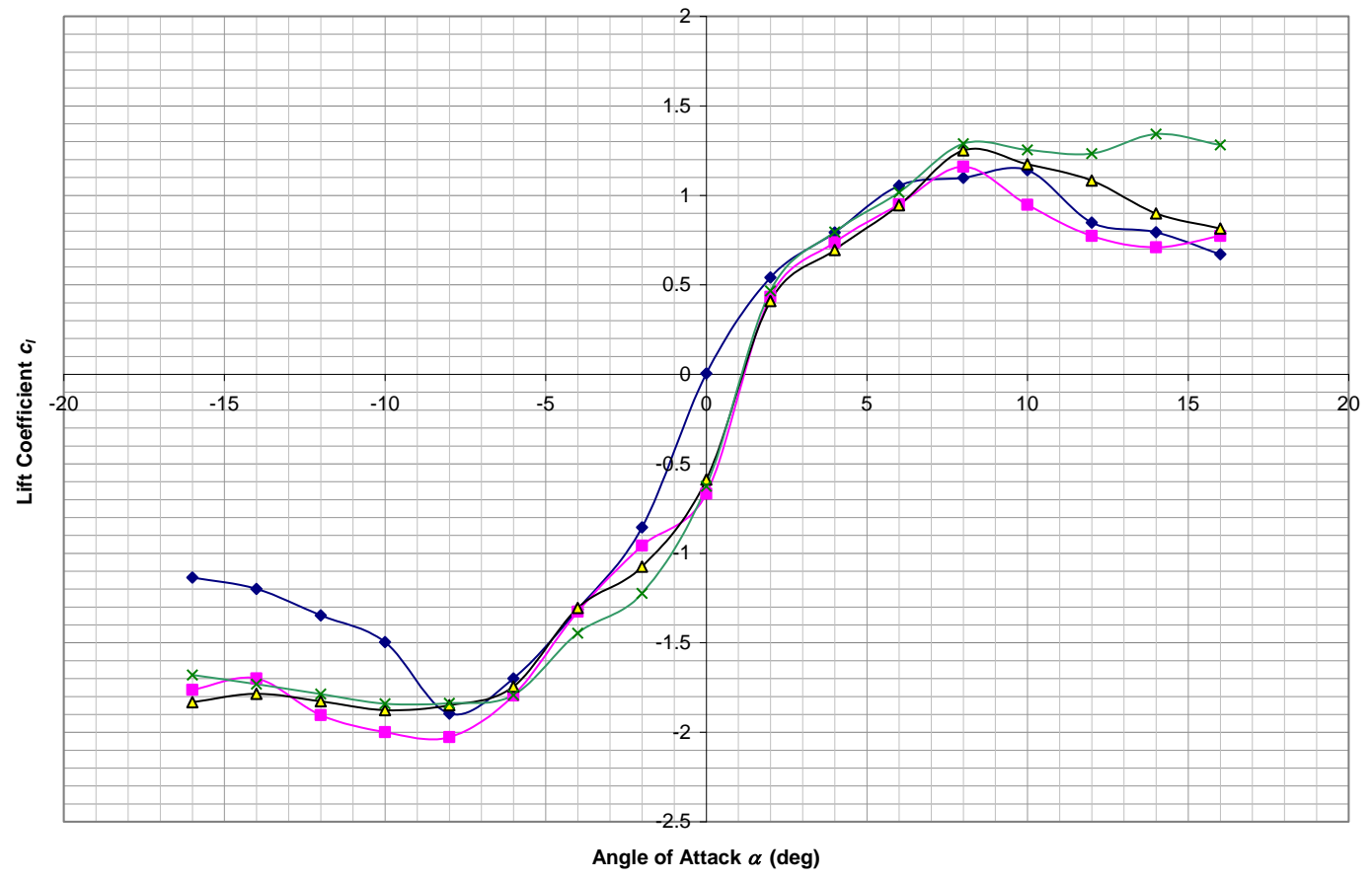

$\rightarrow-$ LE 0 psi, TE 0 psi $\rightarrow$ LE 10 psi, TE 0 psi $\bullet-$ LE 10 psi,TE 10 psi $\rightarrow$ LE 10 psi, TE 30 psi

Figure B.14: Tunnel Velocity $=80 \mathrm{fps}$, Trailing Edge Varying and Leading Edge Blowing at 10 psi, Lift Coefficient vs. Angle of Attack (average tare)

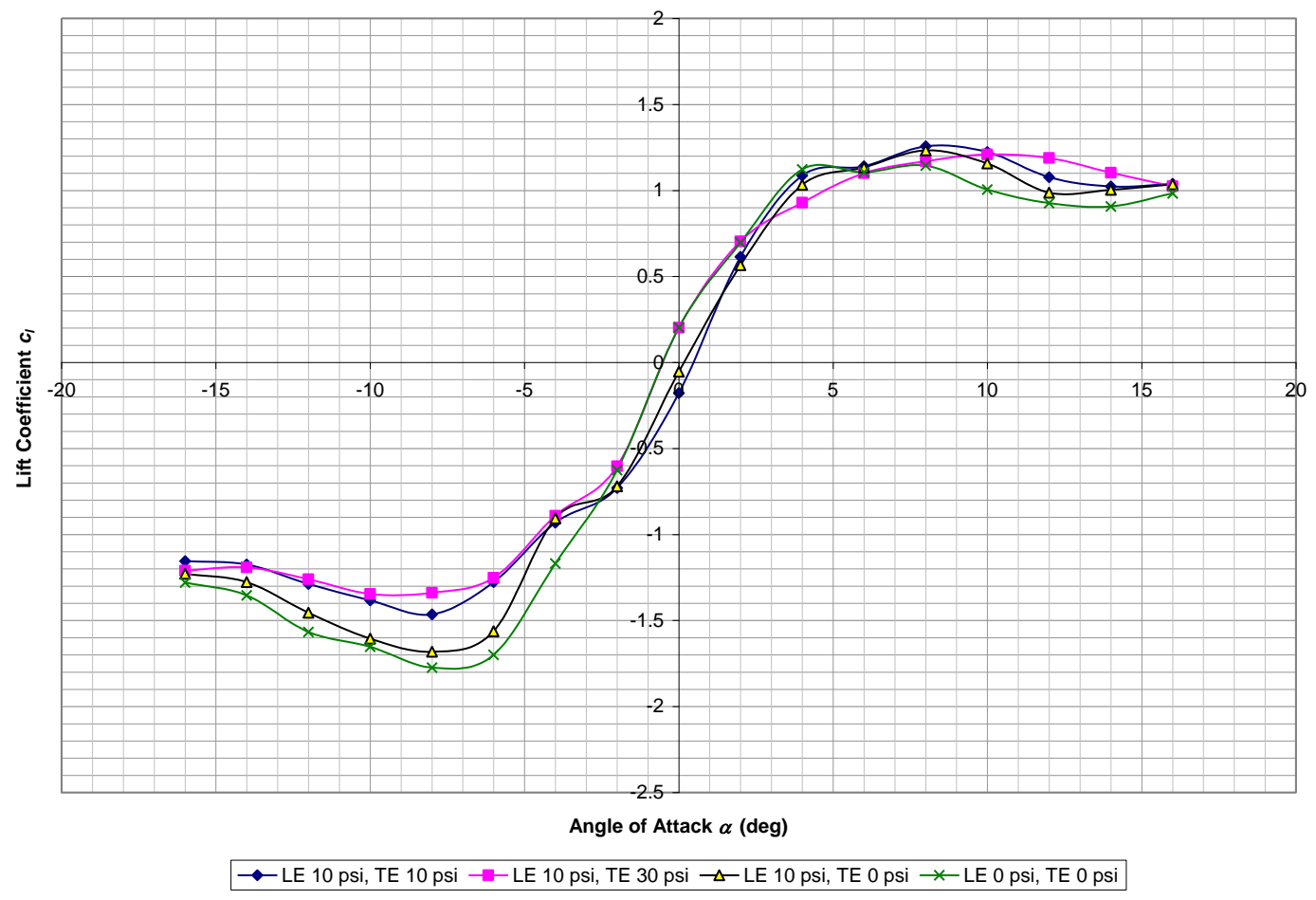

Figure B.15: Tunnel Velocity = $120 \mathrm{fps}$, Trailing Edge Varying and Leading Edge Blowing at $10 \mathrm{psi}$, Lift Coefficient vs. Angle of Attack (average tare) 


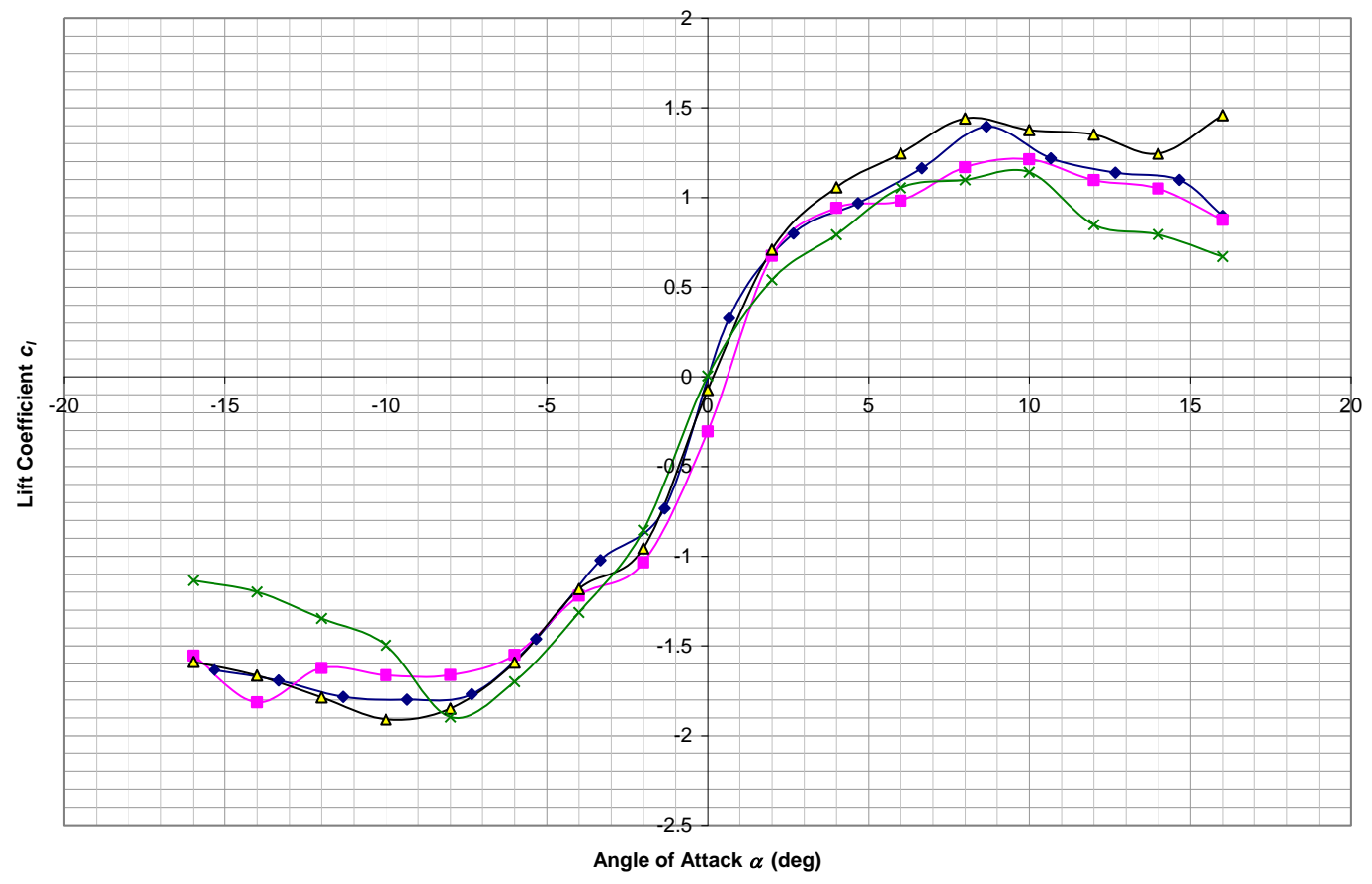

$\rightarrow-$ LE 20 psi, TE 10 psi $\rightarrow$ LE 20 psi, TE 30 psi $\neg$ LE 20 psi, TE 0 psi $\nleftarrow$ LE 0 psi, TE 0 psi

Figure B.16: Tunnel Velocity $=80 \mathrm{fps}$, Trailing Edge Varying and Leading Edge Blowing at 20 psi, Lift Coefficient vs. Angle of Attack (average tare)

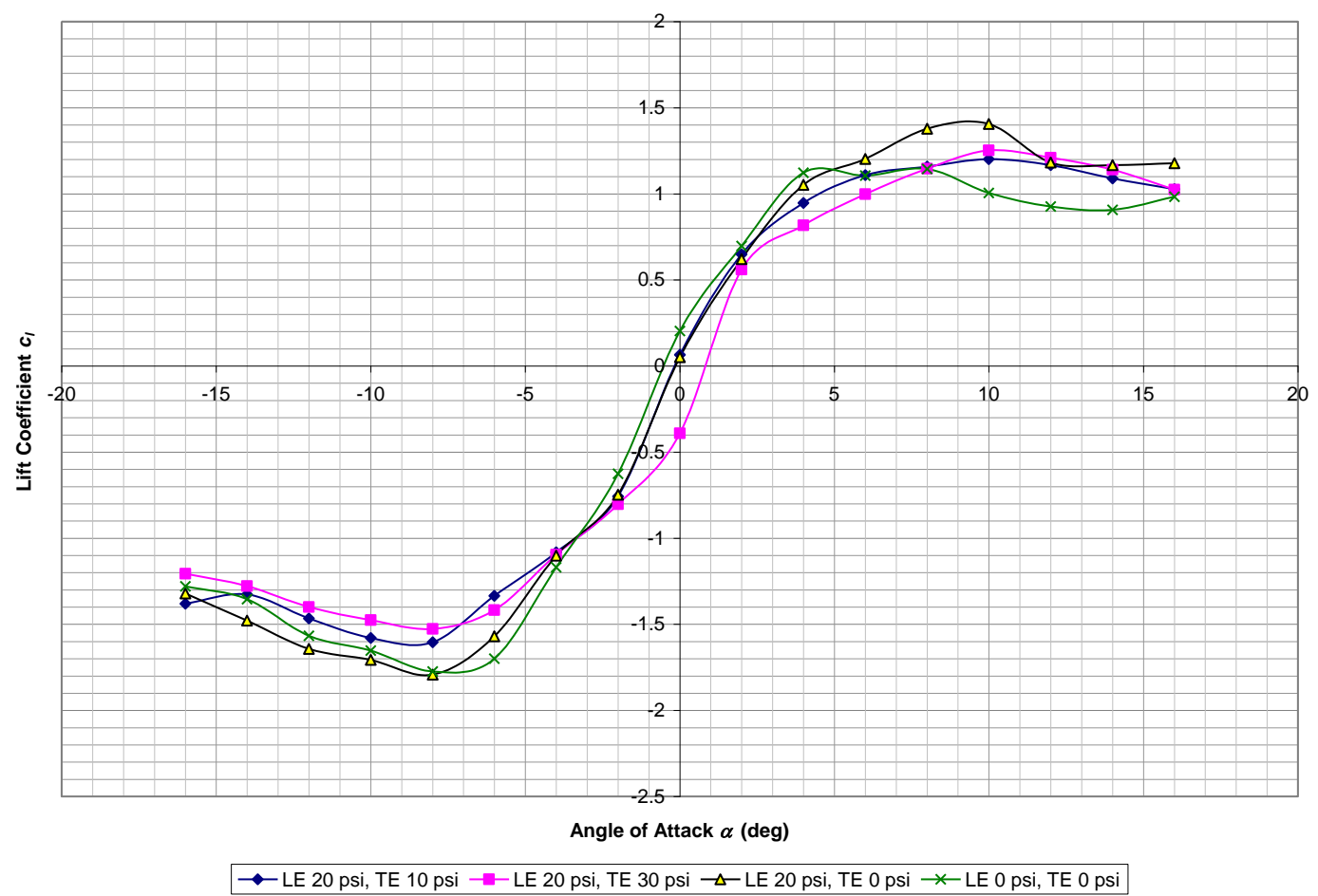

Figure B.17: Tunnel Velocity $=120 \mathrm{fps}$, Trailing Edge Varying and Leading Edge Blowing at 20 psi, Lift Coefficient vs. Angle of Attack (average tare) 


\section{B.3 Lift Coefficient - Trailing Edge Varying - Individual Tares Method}

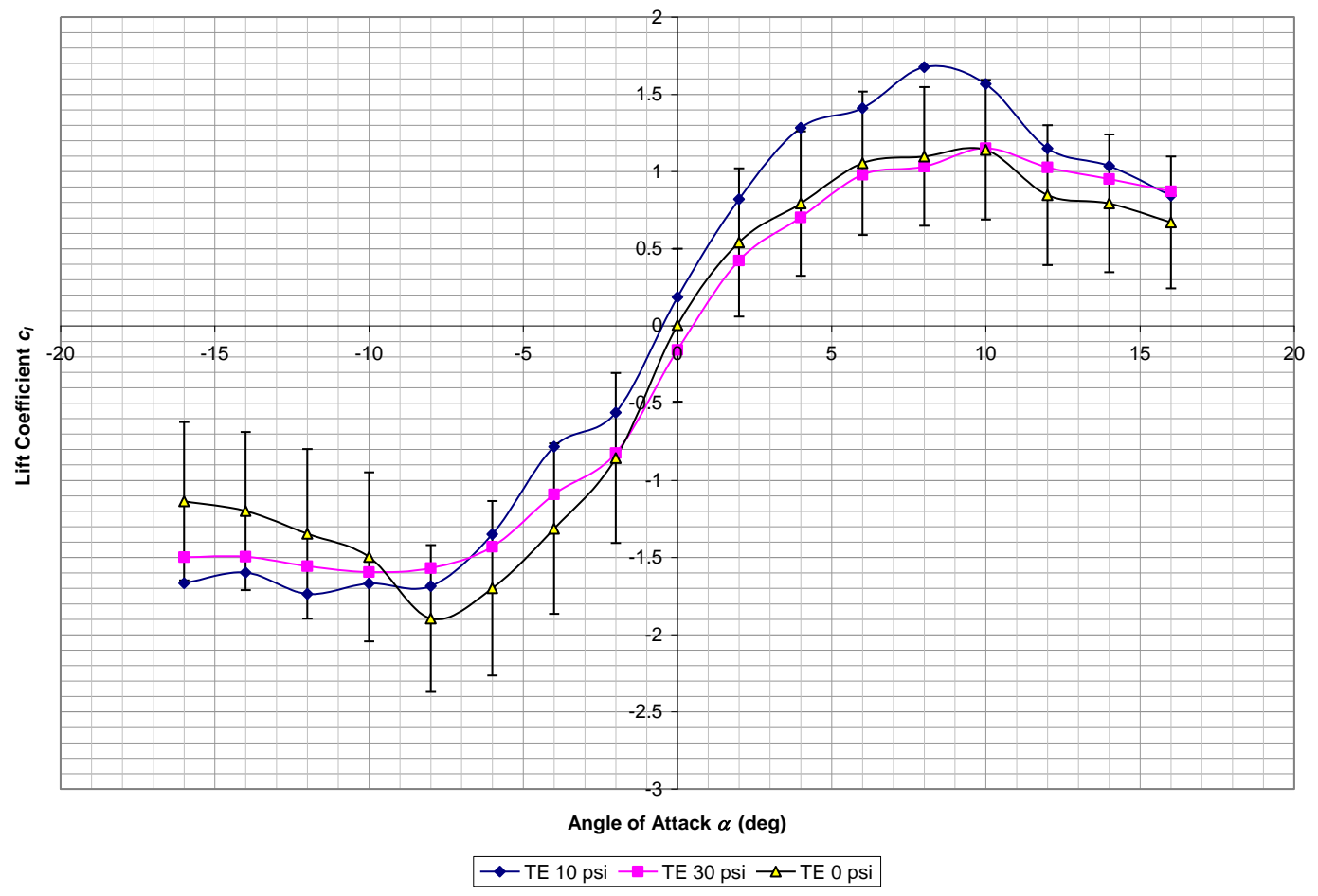

Figure B.18: Tunnel Velocity $=80 \mathrm{fps}$, Trailing Edge Blowing Only, Lift Coefficient vs. Angle of Attack (Error Bars)

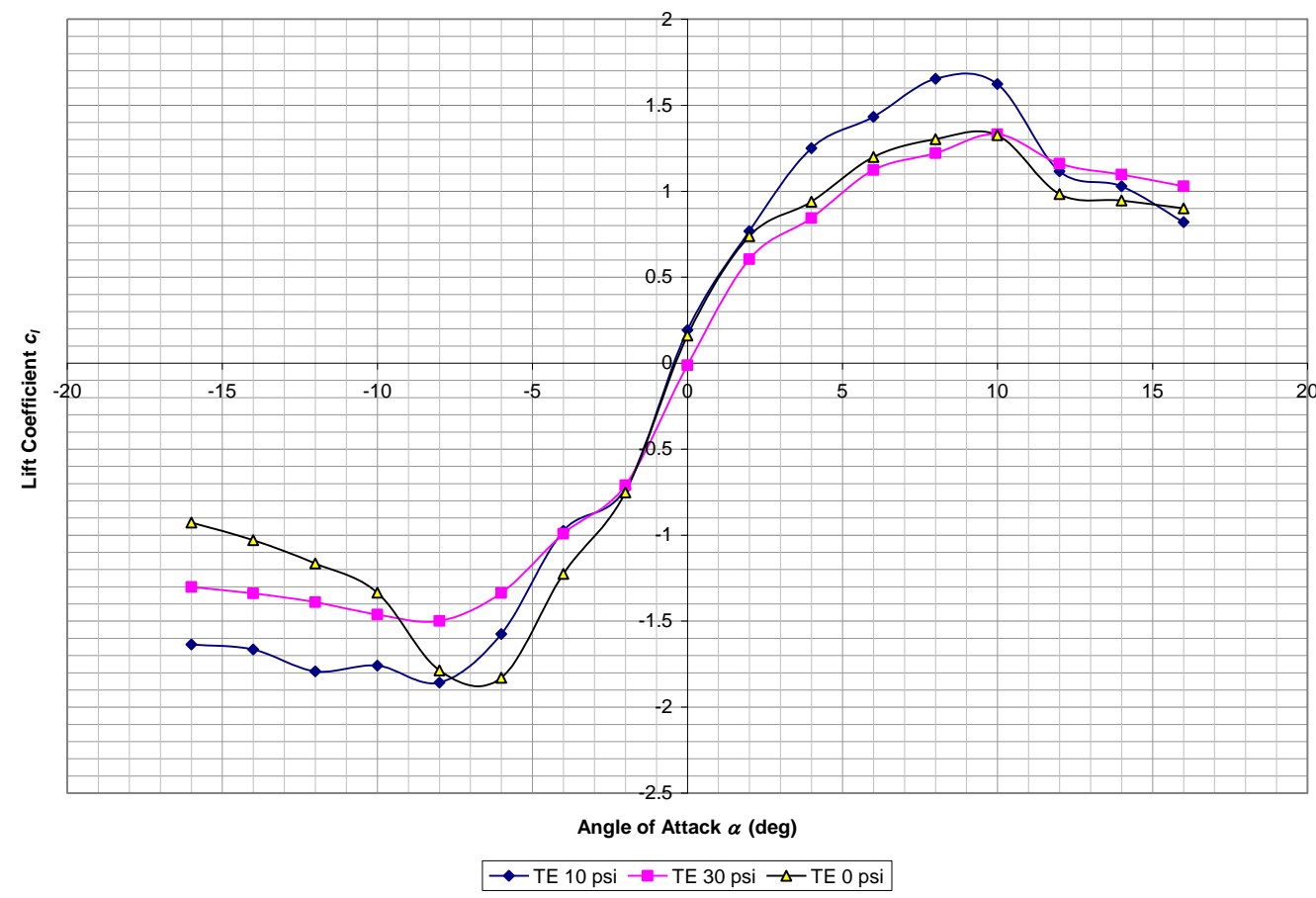

Figure B.19: Tunnel Velocity $=80 \mathrm{fps}$, Trailing Edge Blowing Only, Lift Coefficient vs. Angle of Attack (individual tares) 


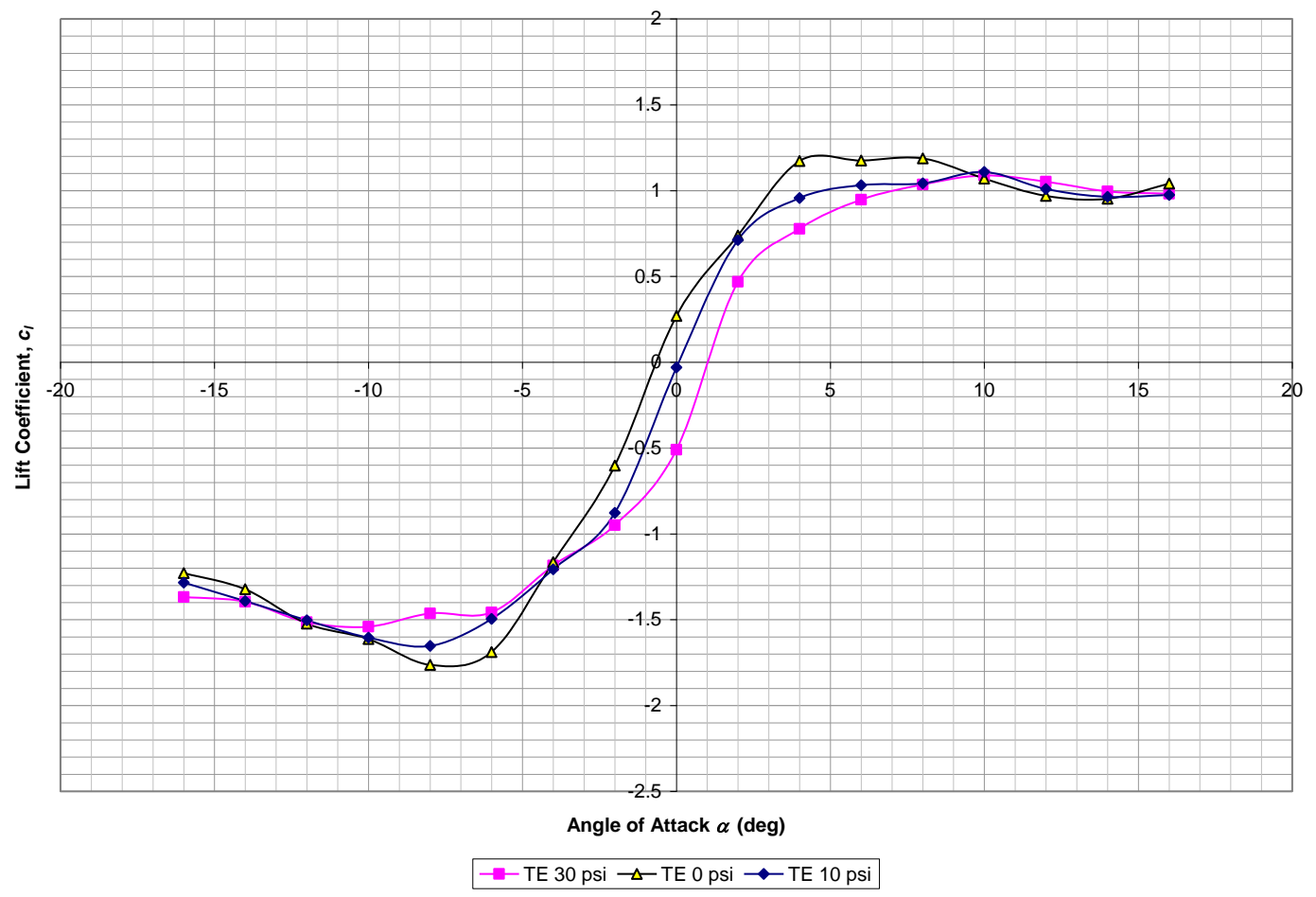

Figure B.20: Tunnel Velocity $=120 \mathrm{fps}$, Trailing Edge Blowing Only, Lift Coefficient vs. Angle of Attack (individual tares)

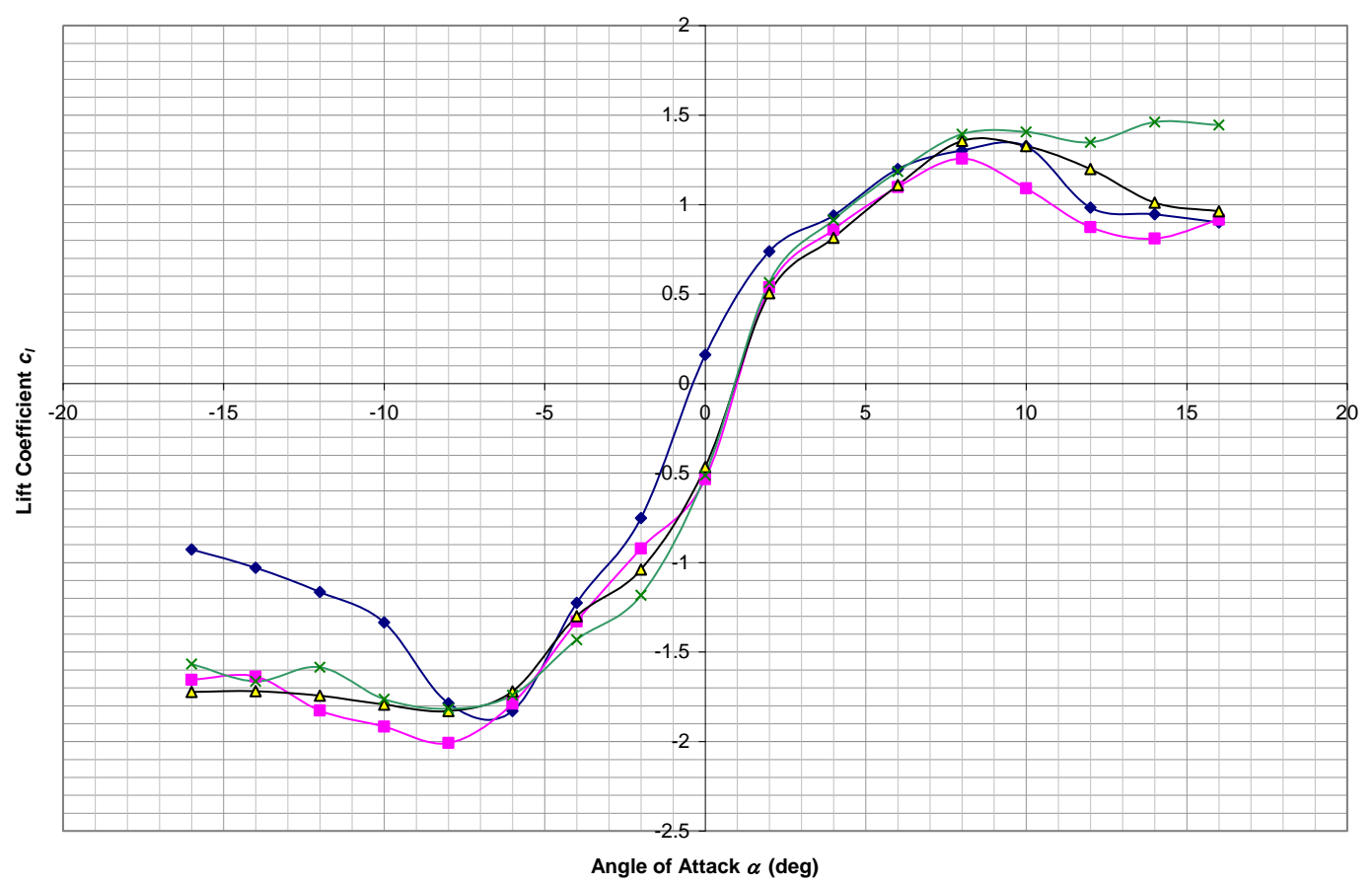

$\rightarrow-$ LE 0 psi, TE 0 psi $\rightarrow-$ LE 10 psi, TE 0 psi $-\triangle-$ LE 10 psi, TE 10 psi $\rightarrow$ LE 10 psi, TE 30 psi

Figure B.21: Tunnel Velocity $=80 \mathrm{fps}$, Trailing Edge Varying and Leading Edge Blowing at $10 \mathrm{psi}$, Lift Coefficient vs. Angle of Attack (individual tares) 


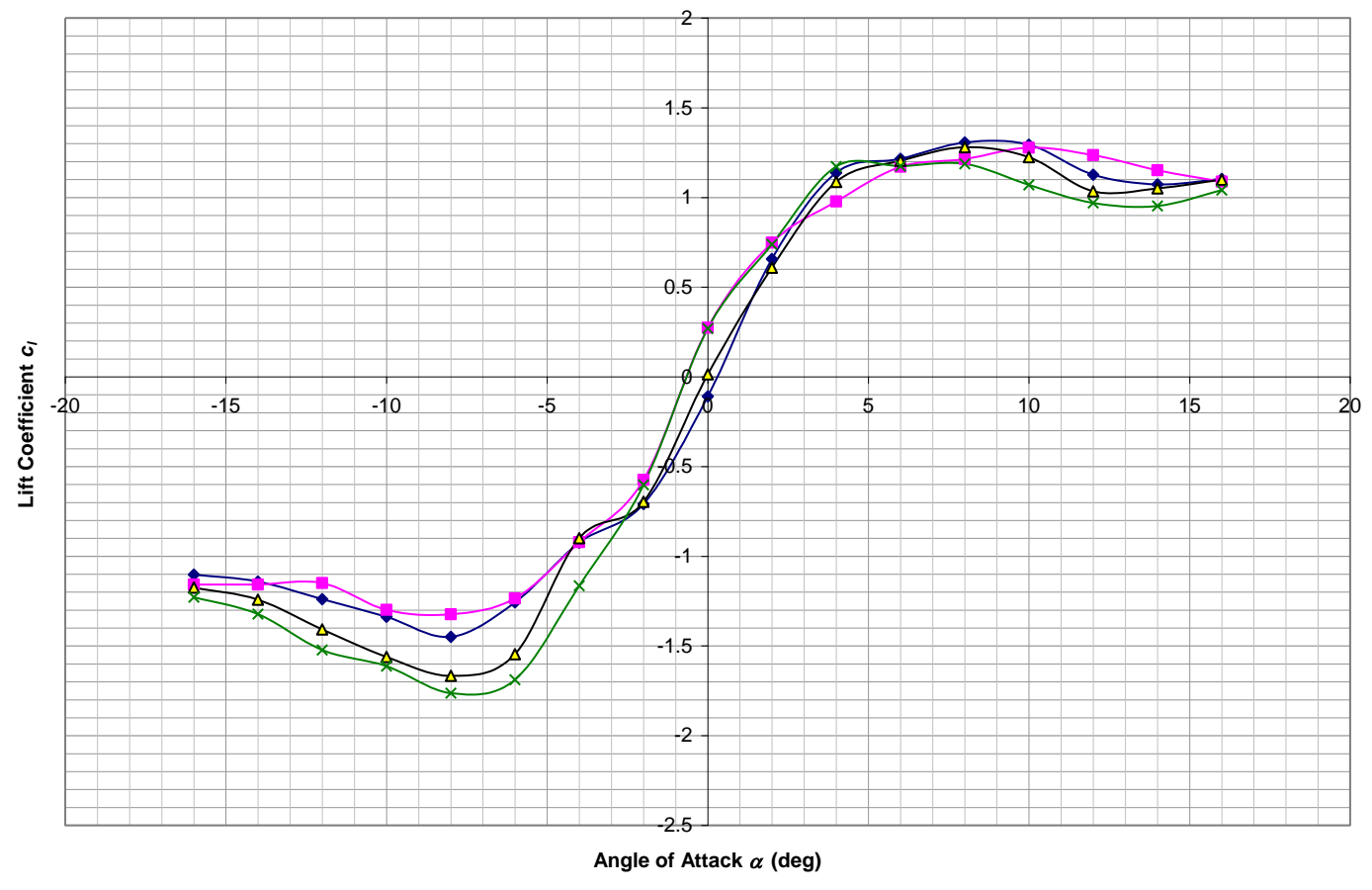

$\rightarrow-$ LE 10 psi, TE 10 psi $\rightarrow$ LE 10 psi, TE 30 psi $\rightarrow-$ LE 10 psi, TE 0 psi $\rightarrow$ LE 0 psi, TE 0 psi

Figure B.22: Tunnel Velocity $=120 \mathrm{fps}$, Trailing Edge Varying and Leading Edge Blowing at $10 \mathrm{psi}$, Lift Coefficient vs. Angle of Attack (individual tares)

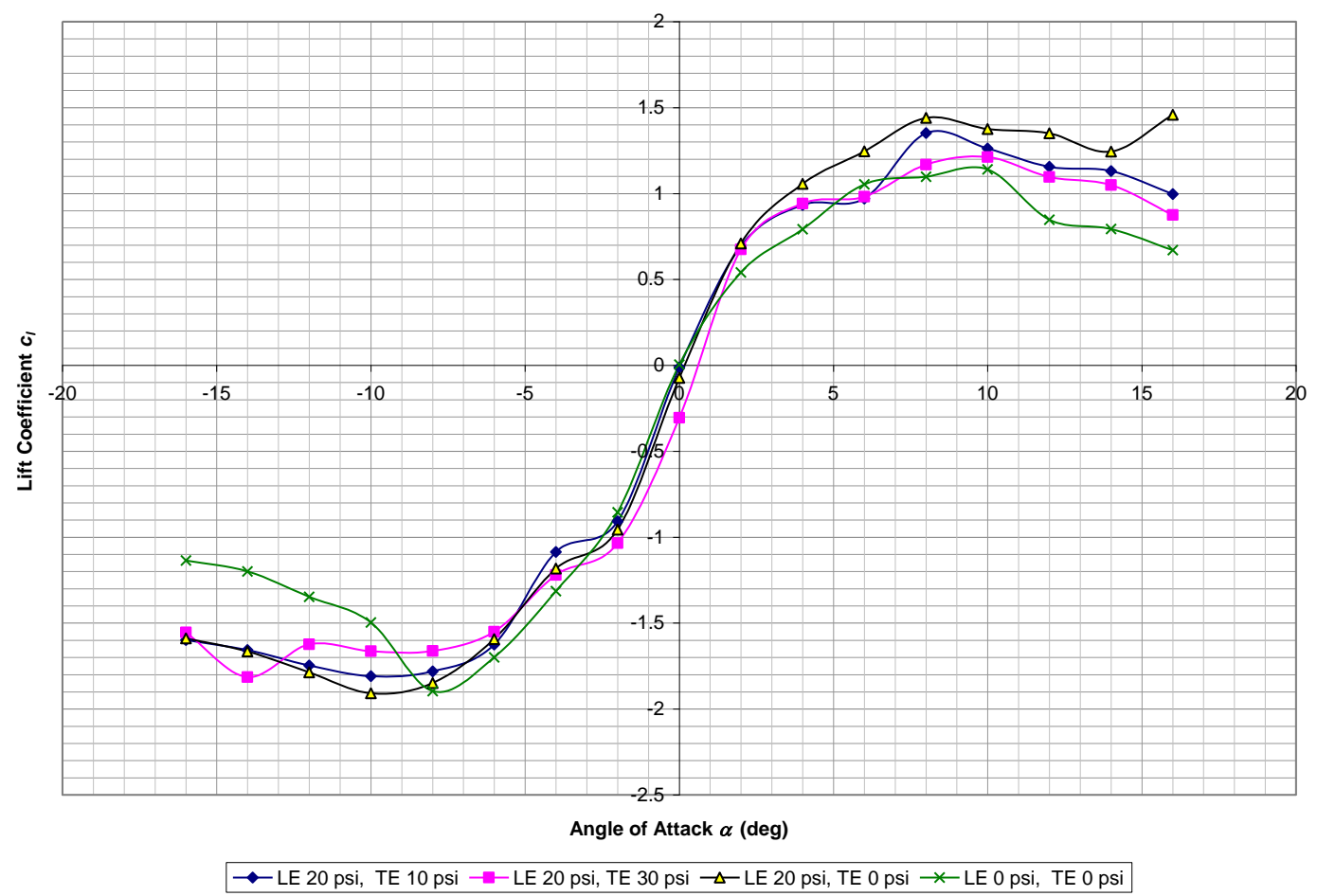

Figure B.23: Tunnel Velocity $=80 \mathrm{fps}$, Trailing Edge Varying and Leading Edge Blowing at $20 \mathrm{psi}$, Lift Coefficient vs. Angle of Attack (individual tares) 


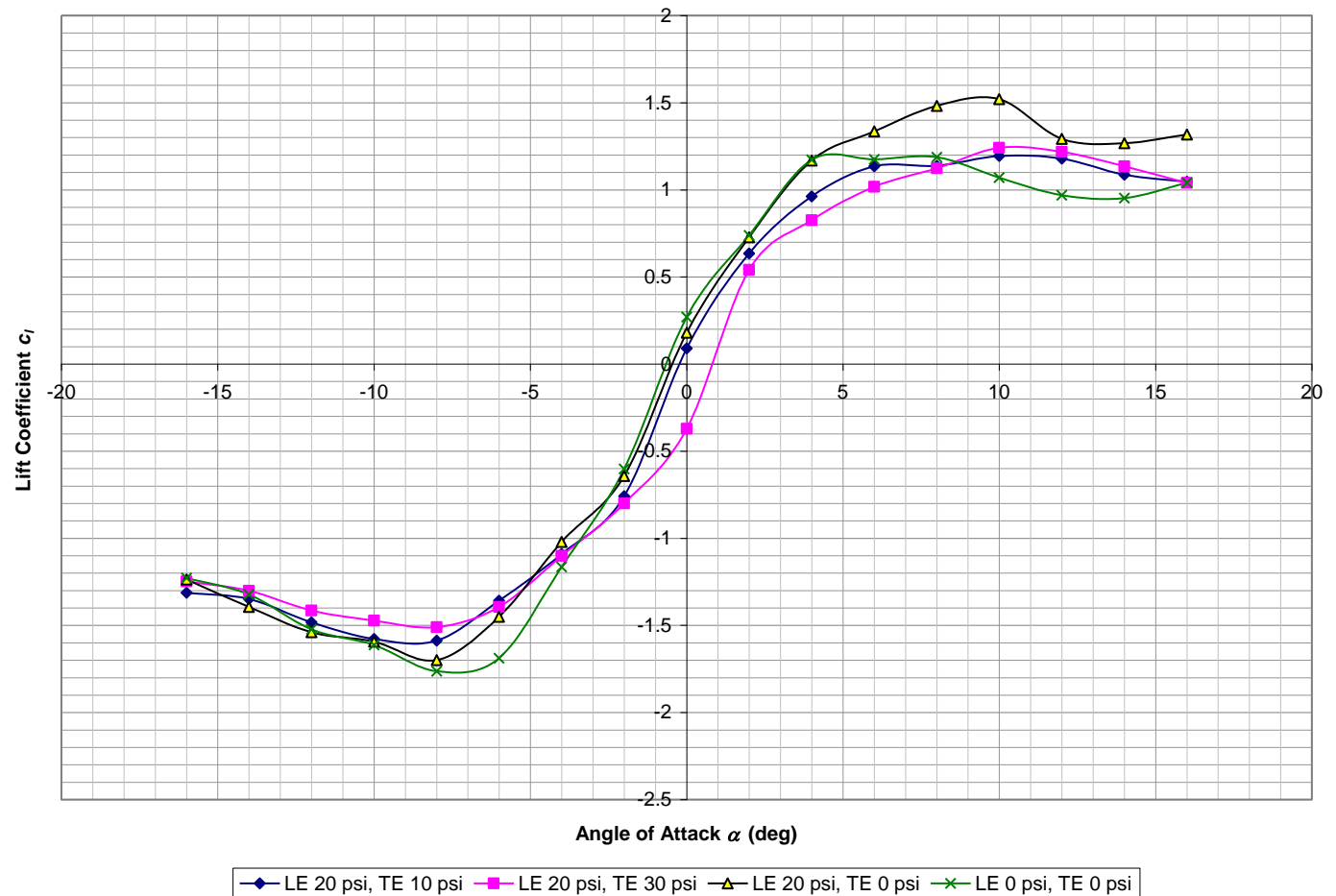

Figure B.24: Tunnel Velocity $=120$ fps, Trailing Edge Varying and Leading Edge Blowing at 20 psi, Lift Coefficient vs. Angle of Attack (individual tares) 


\section{B.4 Lift Coefficient Change - Trailing Edge Varying}

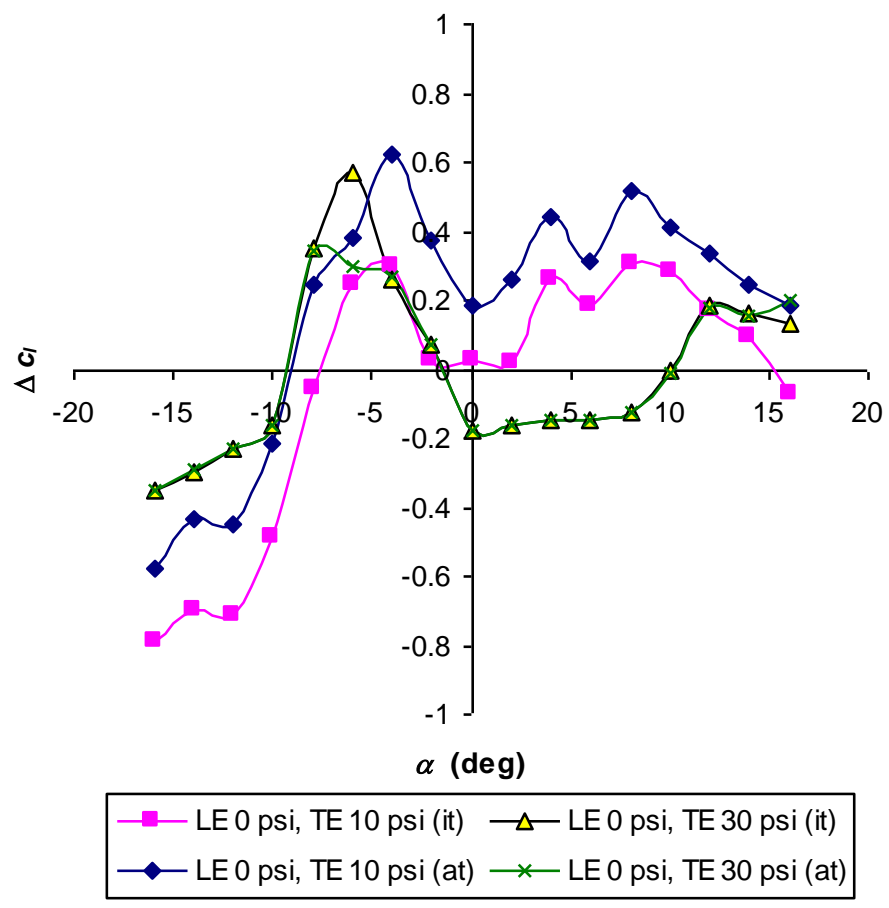

Figure B.25: Tunnel Velocity = $80 \mathrm{fps}$, Change in Lift Coefficient vs. Angle of Attack, Trailing Edge Blowing Only

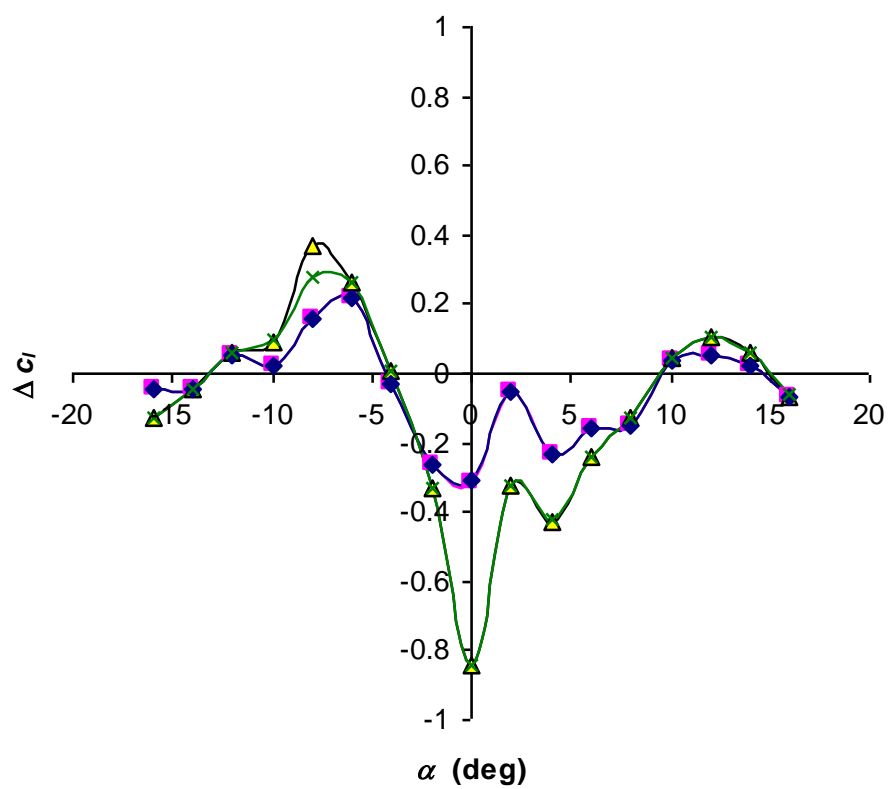

$\rightarrow-$ LE0 psi, TE 10 psi (it) $\longrightarrow-$ LE0 psi, TE30 psi (it)

- LE 0 psi, TE 10 psi (at) $\rightarrow$ LE 0 psi, TE 30 psi (at) 
Figure B.26: Tunnel Velocity = 120 fps, Change in Lift Coefficient vs. Angle of Attack, Trailing Edge Blowing Only

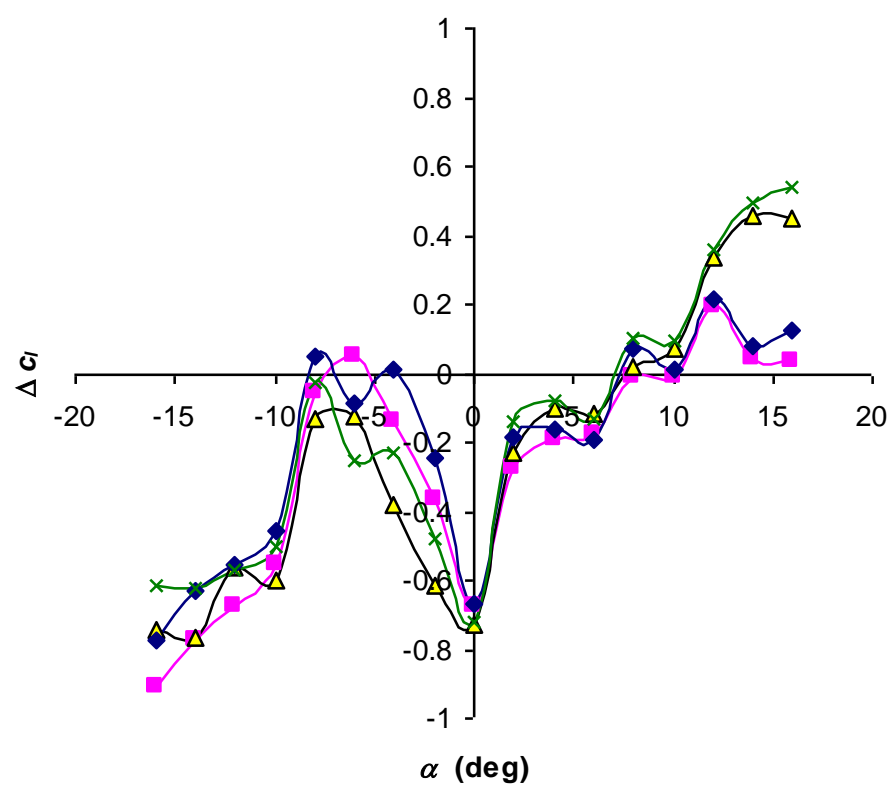

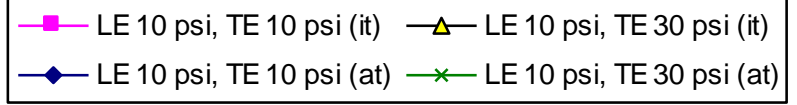

Figure B.27: Tunnel Velocity = $80 \mathrm{fps}$, Change in Lift Coefficient vs. Angle of Attack, Trailing Edge Varying, Leading Edging Blowing at 10 psi

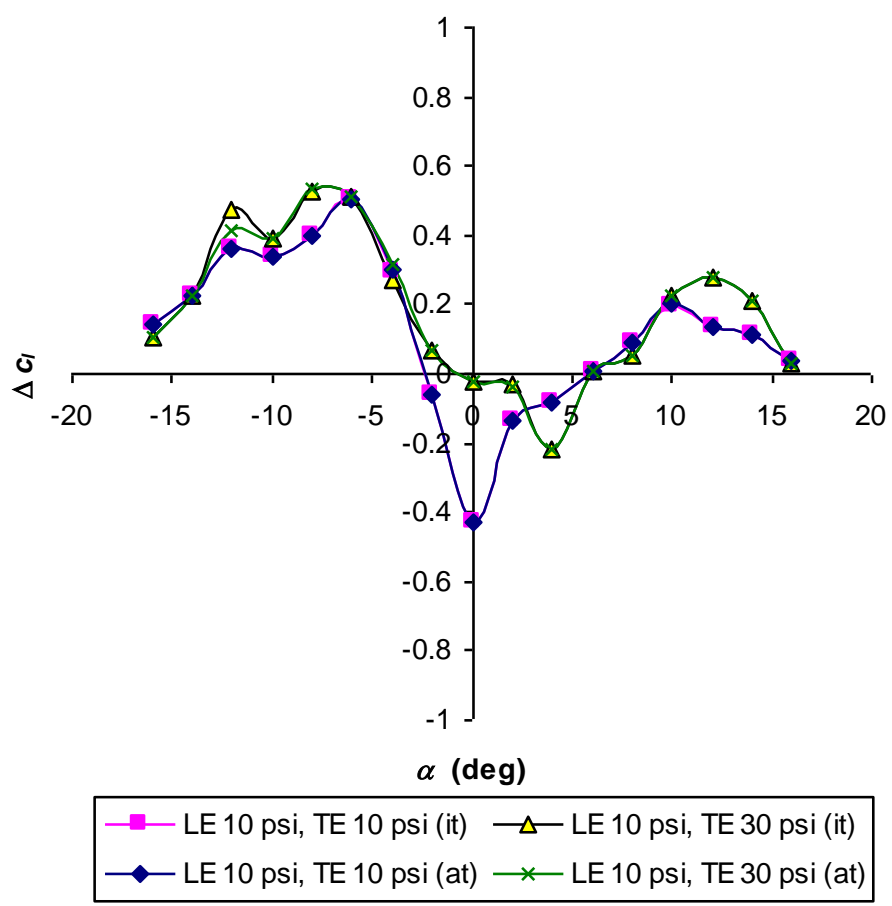

Figure B.28: Tunnel Velocity $=120 \mathrm{fps}$, Change in Lift Coefficient vs. Angle of Attack, Trailing Edge Varying, Leading Edging Blowing at 10 psi 

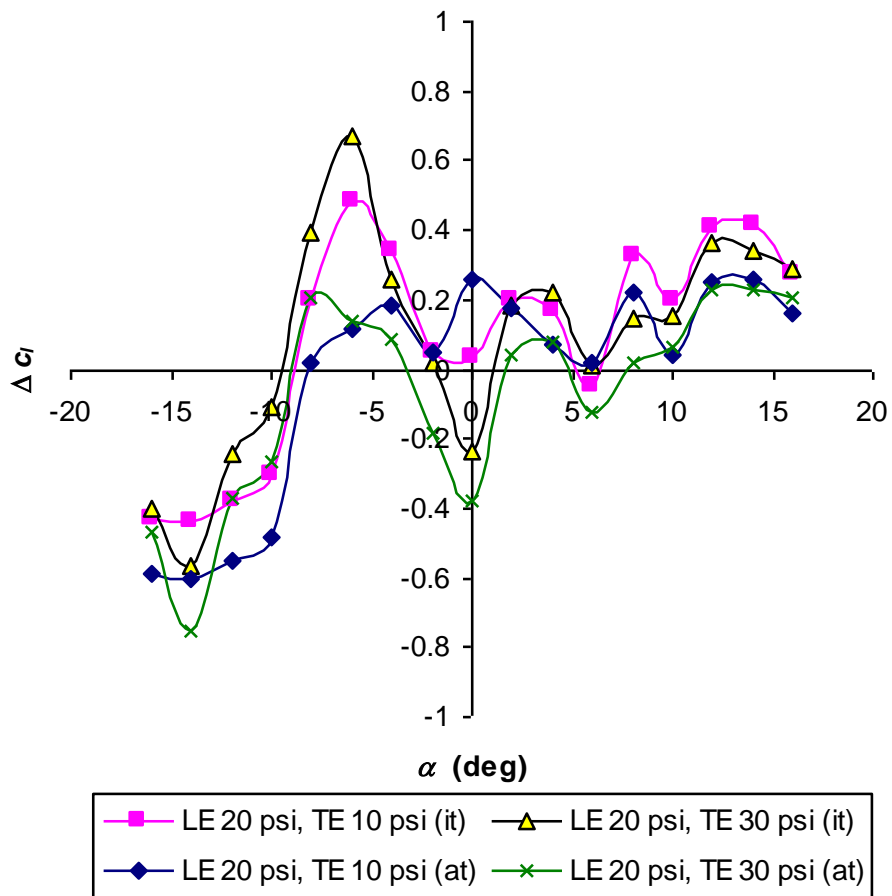

Figure B.29: Tunnel Velocity $=80 \mathrm{fps}$, Change in Lift Coefficient vs. Angle of Attack, Trailing Edge Varying, Leading Edging Blowing at 20 psi

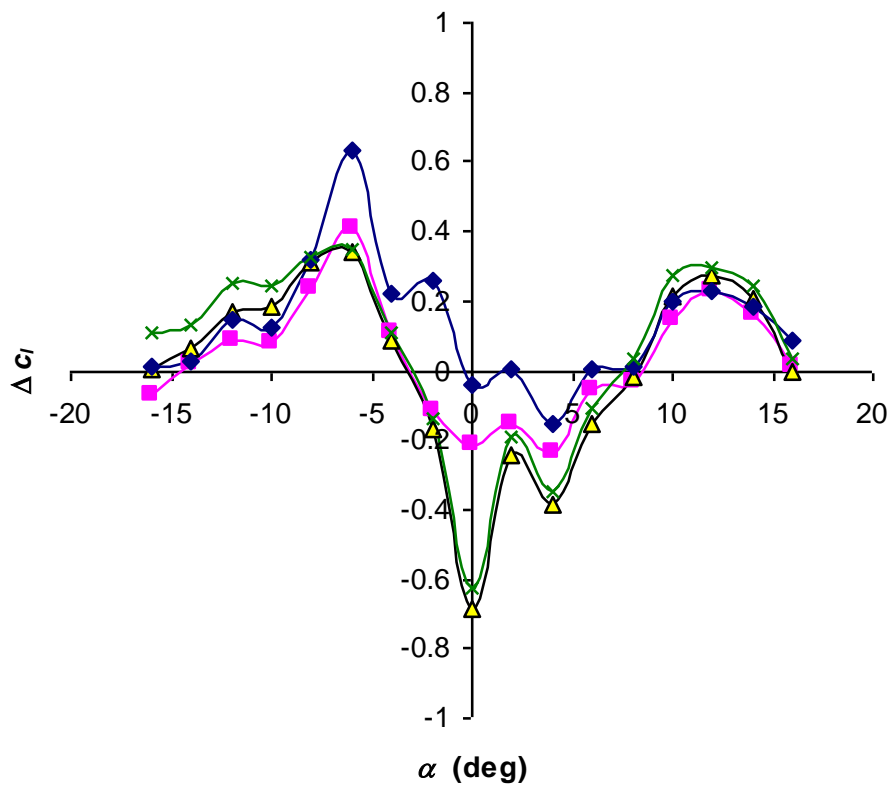

$\longrightarrow$ LE 20 psi, TE 10 psi (it) $\longrightarrow$ LE20 psi, TE 30 psi (it)
$\multimap-$ LE20 psi, TE 10 psi (at) $\longrightarrow$ LE20 psi, TE 30 psi (at)

Figure B.30: Tunnel Velocity = 120 fps, Change in Lift Coefficient vs. Angle of Attack, Trailing Edge Varying, Leading Edging Blowing at 20 psi 


\section{B.5 Lift Coefficient - Leading Edge Varying - Average Tare Method}

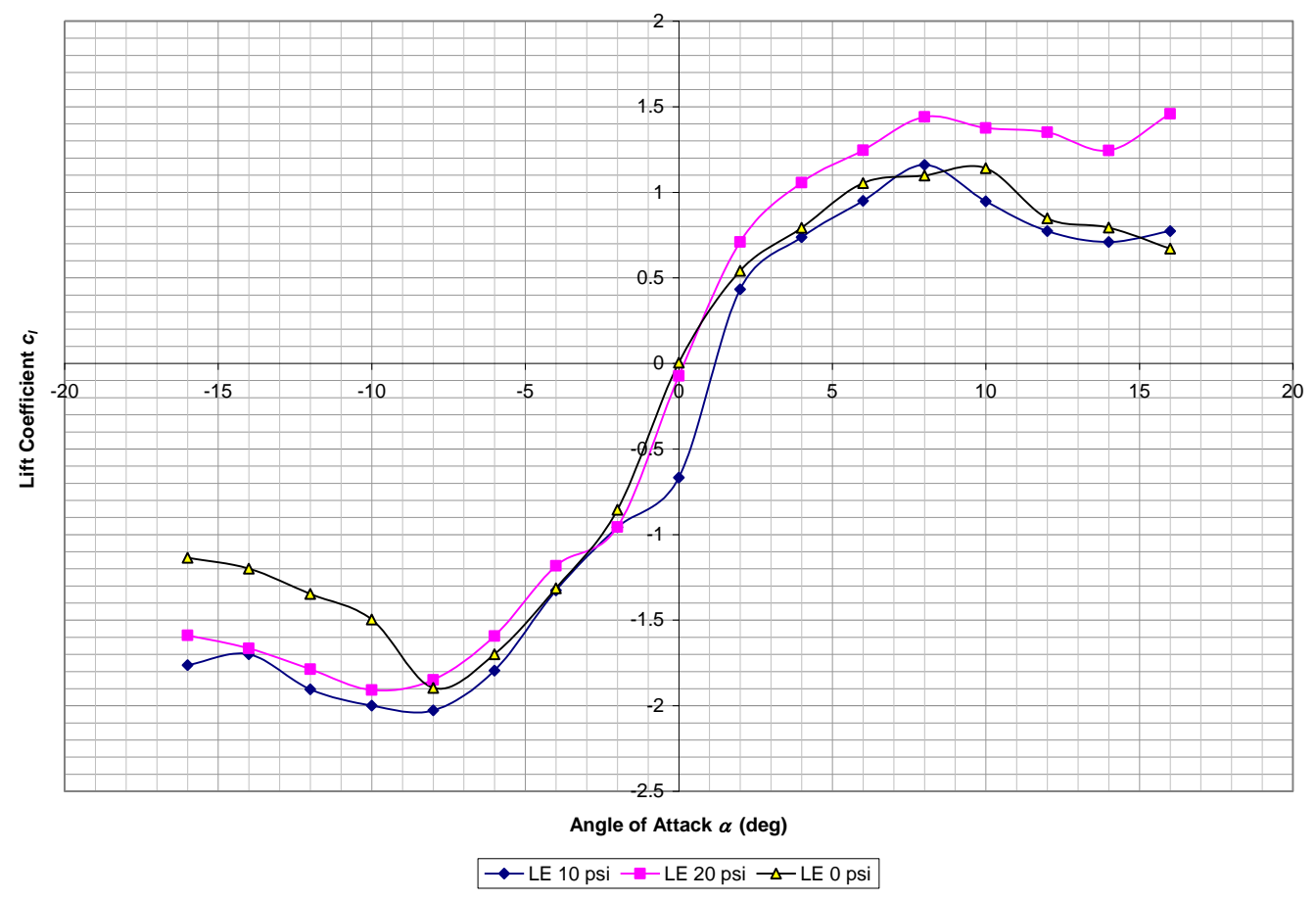

Figure B.31: Tunnel Velocity = $80 \mathrm{fps}$, Change in Lift Coefficient vs. Angle of Attack, Leading Edge Blowing Only (average tare)

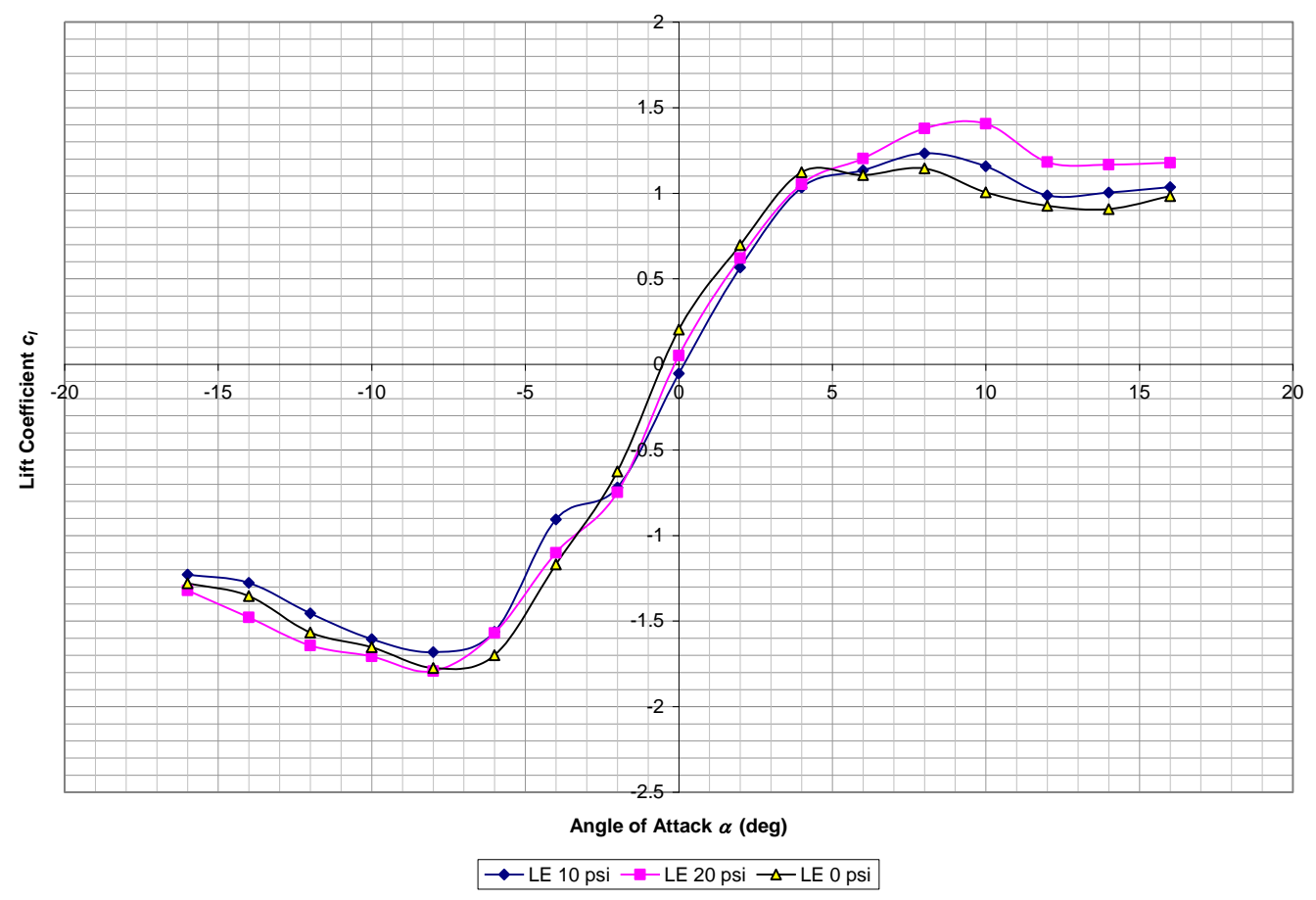

Figure B.32: Tunnel Velocity $=120$ fps, Change in Lift Coefficient vs. Angle of Attack, Leading Edge Blowing Only (average tare) 


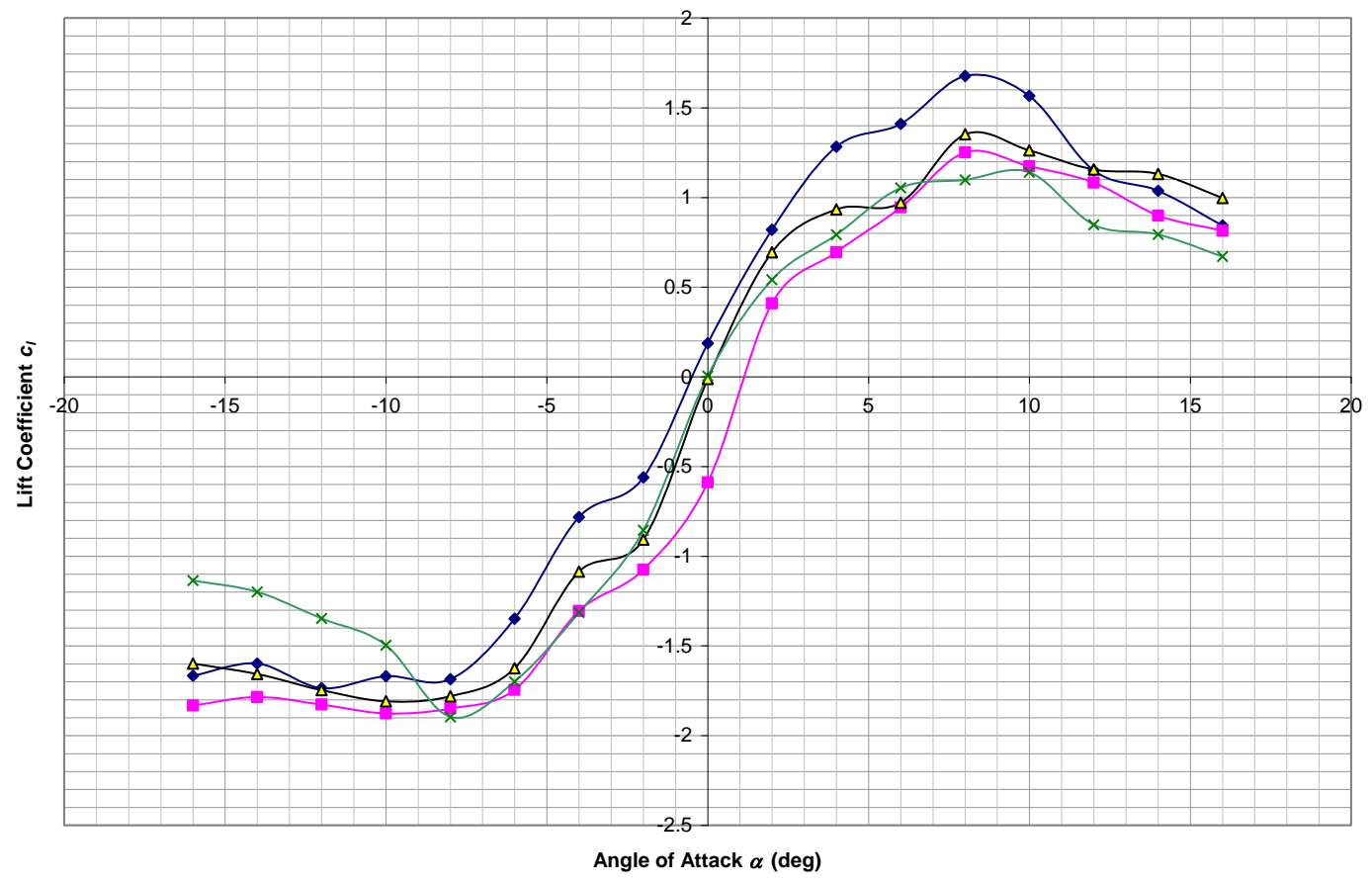

$\rightarrow-$ LE 0 psi, TE 10 psi $\rightarrow-$ LE 10 psi, TE 10 psi $\rightarrow-$ LE 20 psi,TE 10 psi $\rightarrow$ LE 0 psi, TE 0 psi

Figure B.33: Tunnel Velocity $=80 \mathrm{fps}$, Change in Lift Coefficient vs. Angle of Attack, Leading Edge Varying, Trailing Edge Blowing at 10 psi (average tare)

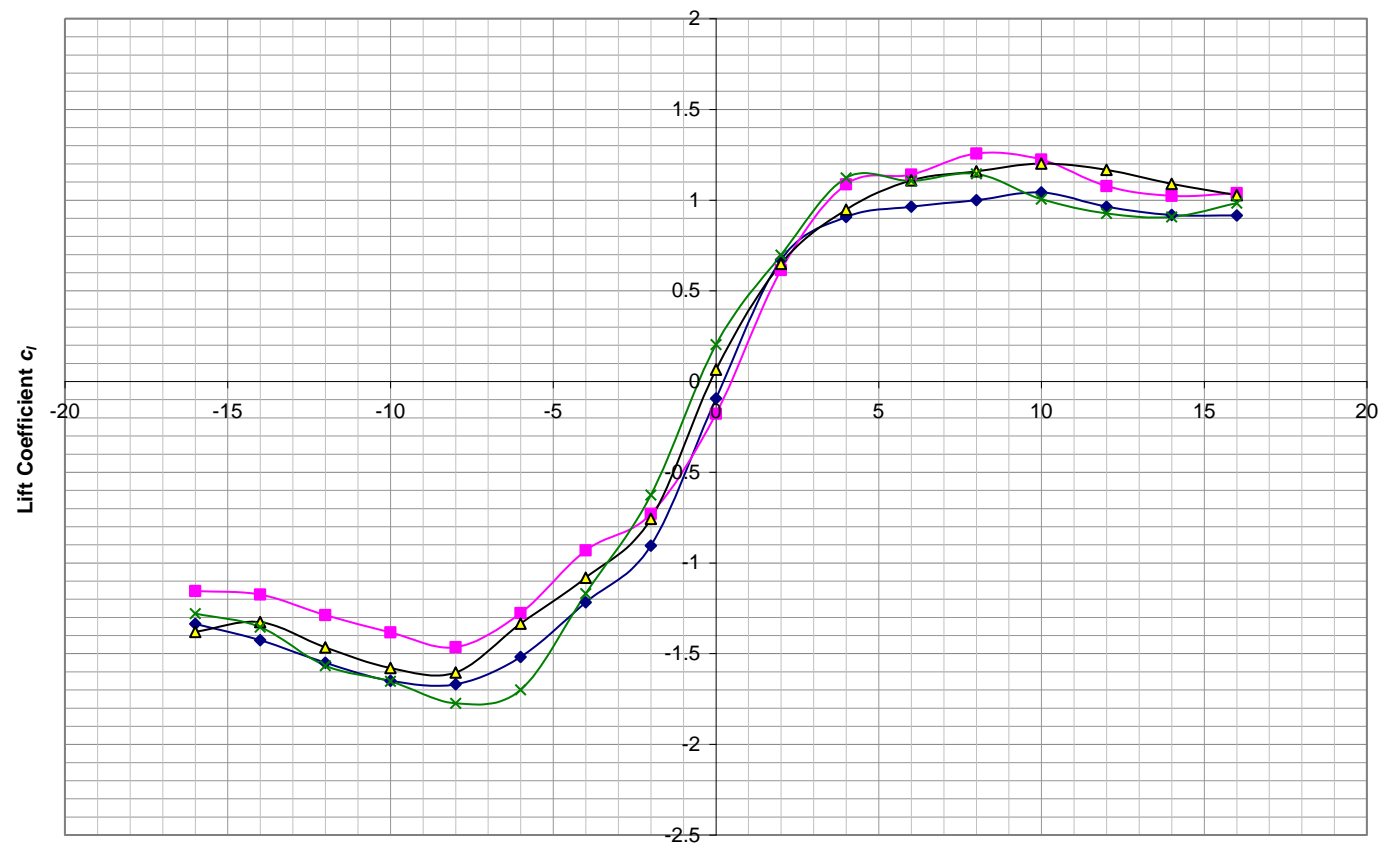

Angle of Attack $\alpha(\mathrm{deg})$

LE 0 psi, TE 10 psi $\rightarrow$ LE 10 psi, TE 10 psi $\neg-$ LE 20 psi, TE 10 psi $¥$ LE 0 psi, TE 0 psi

Figure B.34: Tunnel Velocity $=120$ fps, Change in Lift Coefficient vs. Angle of Attack, Leading Edge Varying, Trailing Edge Blowing at 10 psi (average tare) 


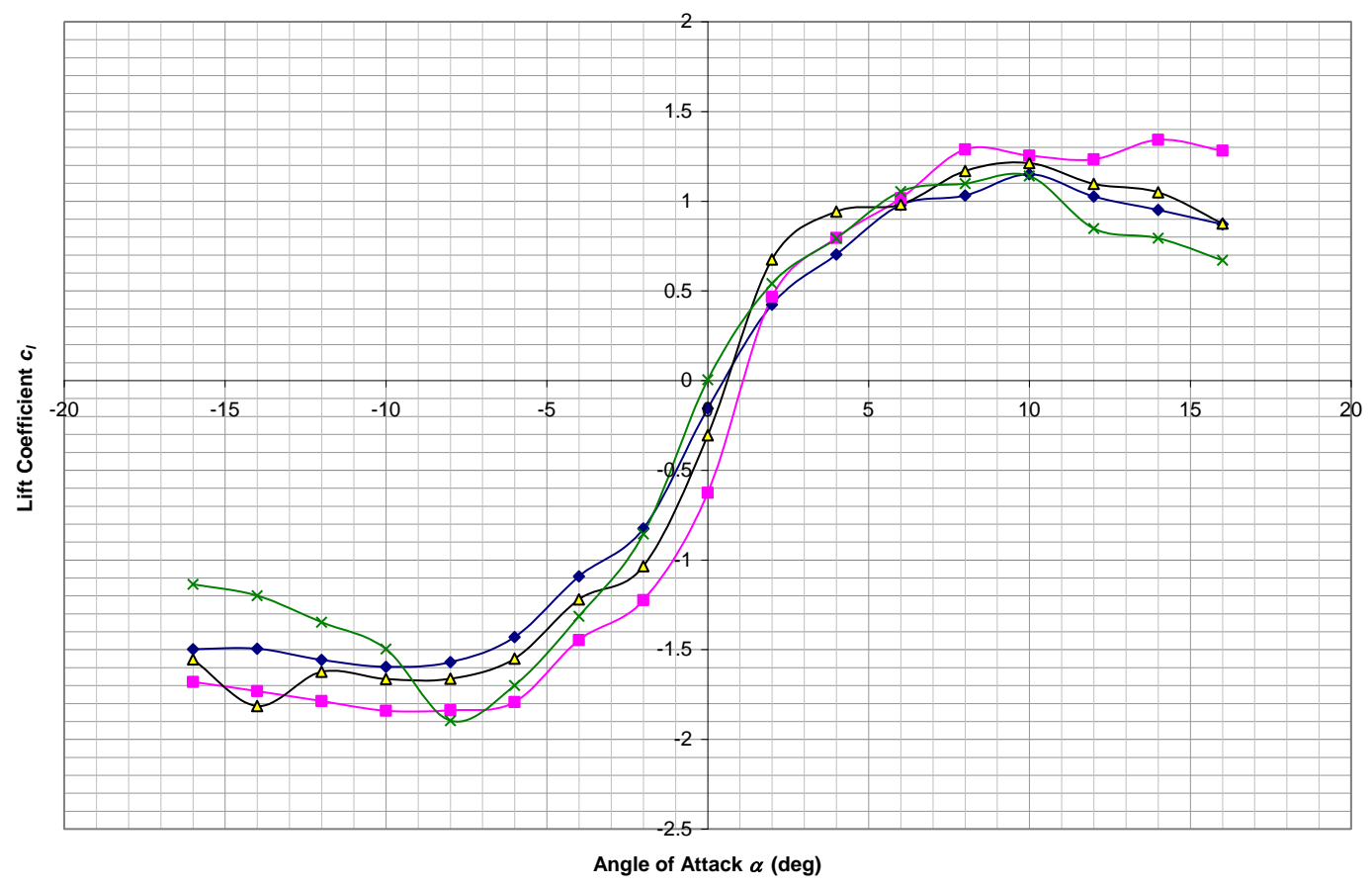

$\rightarrow-$ LE 0 psi, TE 30 psi $\rightarrow$ LE 10 psi, TE 30 psi $\neg$ LE 20 psi, TE 30 psi $¥$ LE 0 psi, TE 0 psi

Figure B.35: Tunnel Velocity $=\mathbf{8 0} \mathrm{fps}$, Change in Lift Coefficient vs. Angle of Attack, Leading Edge Varying, Trailing Edge Blowing at 30 psi (average tare)

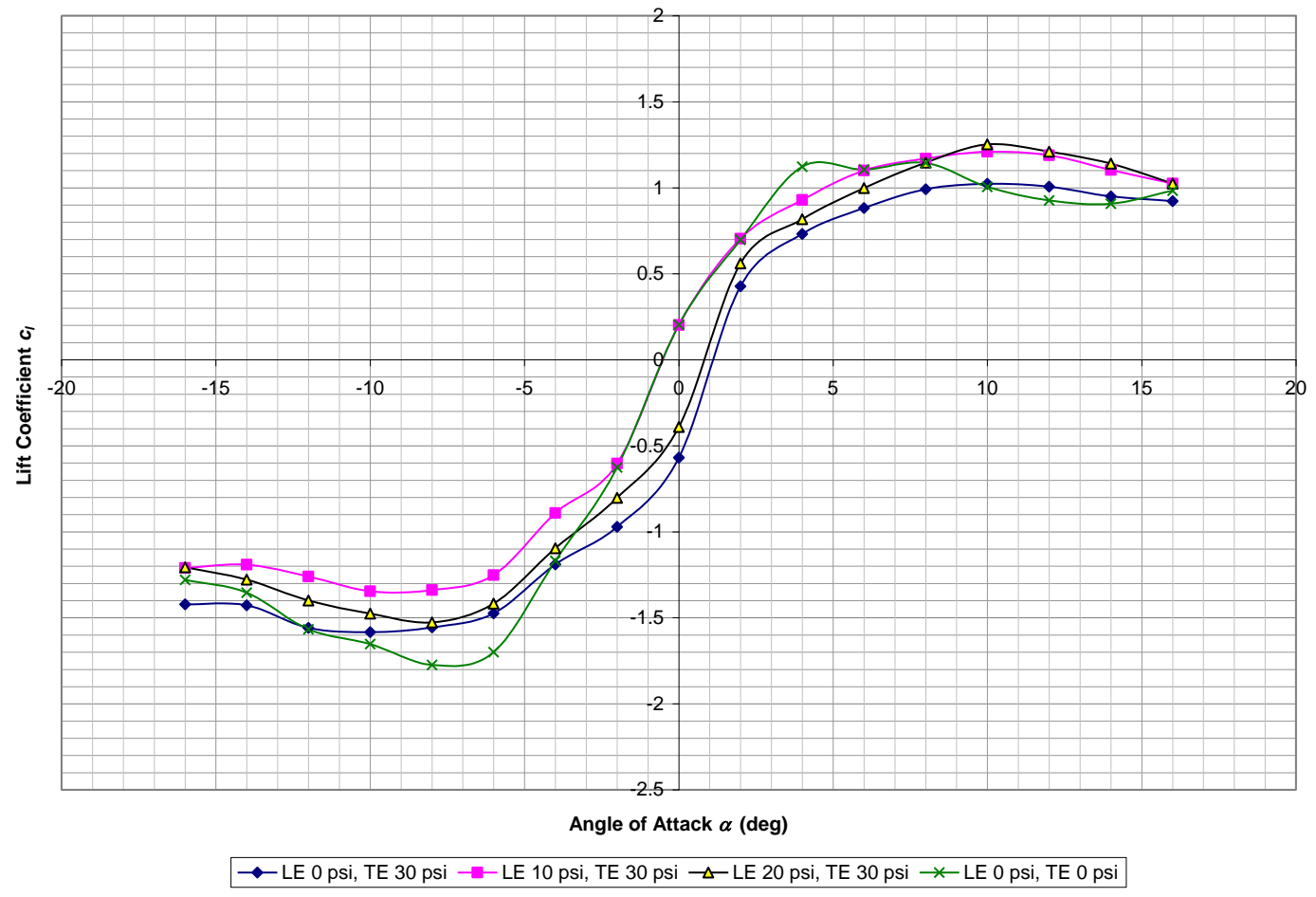

Figure B.36: Tunnel Velocity $=120 \mathrm{fps}$, Change in Lift Coefficient vs. Angle of Attack, Leading Edge Varying, Trailing Edge Blowing at 30 psi (average tare) 


\section{B.6 Lift Coefficient Change - Leading Edge Varying}

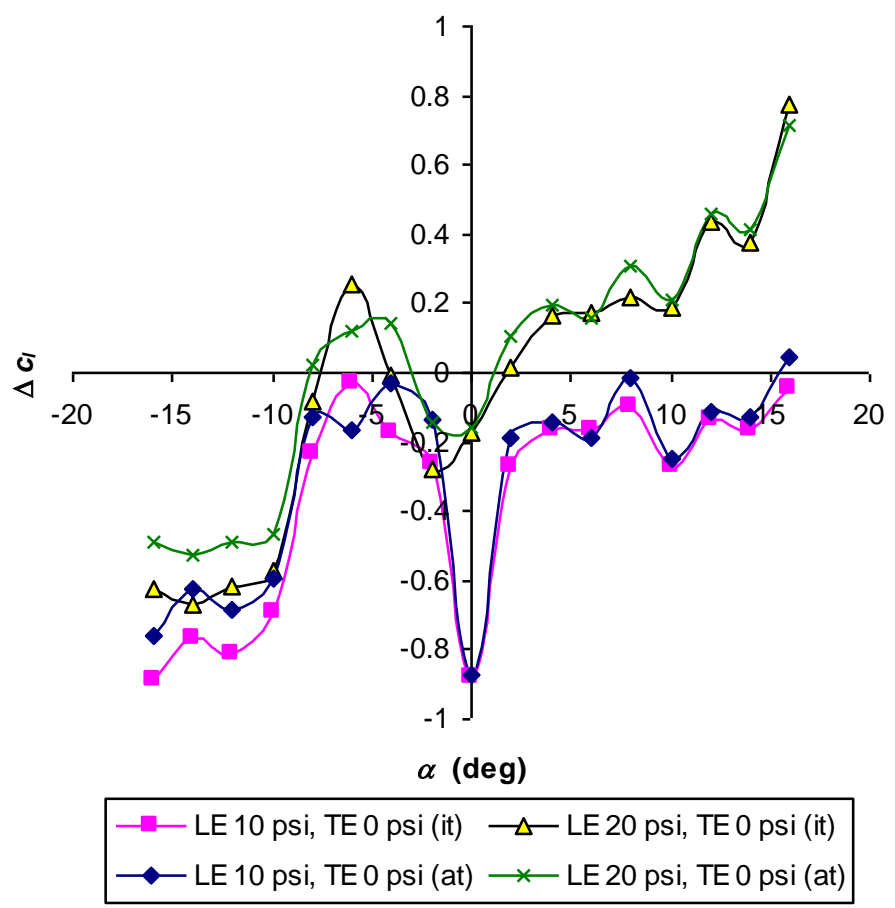

Figure B.37: Tunnel Velocity $=\mathbf{8 0} \mathrm{fps}$, Change in lift coefficient, Leading Edge Blowing Only

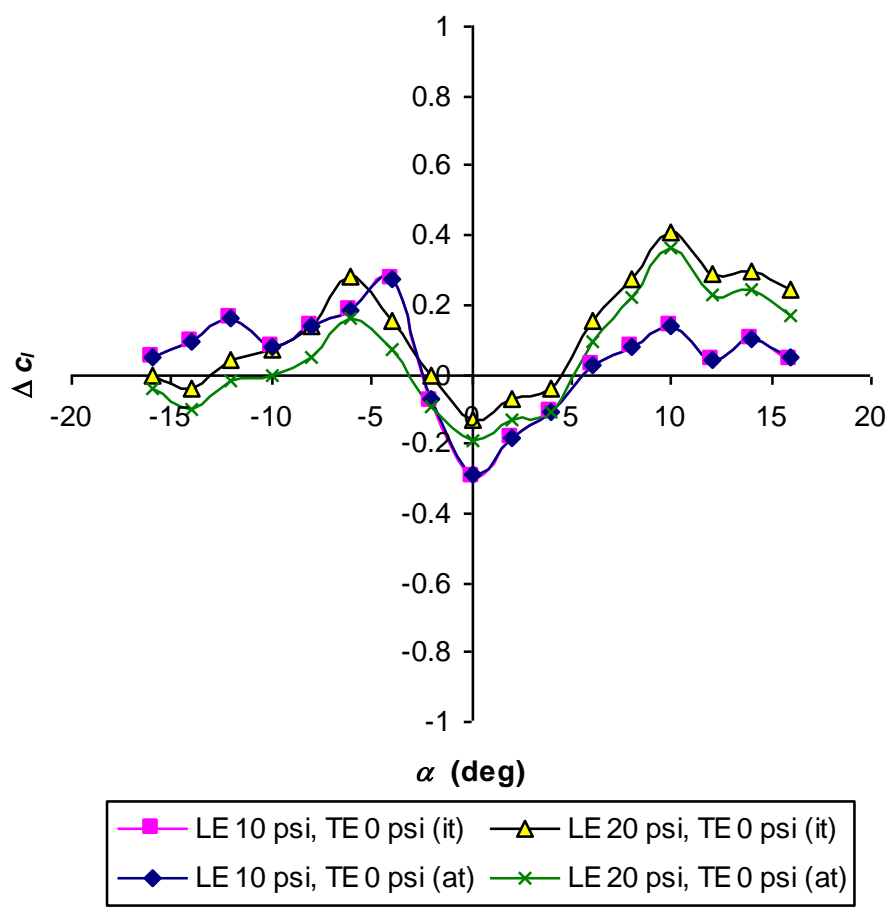

Figure B.38: Tunnel Velocity $=120 \mathrm{fps}$, Change in lift coefficient, Leading Edge Blowing Only 


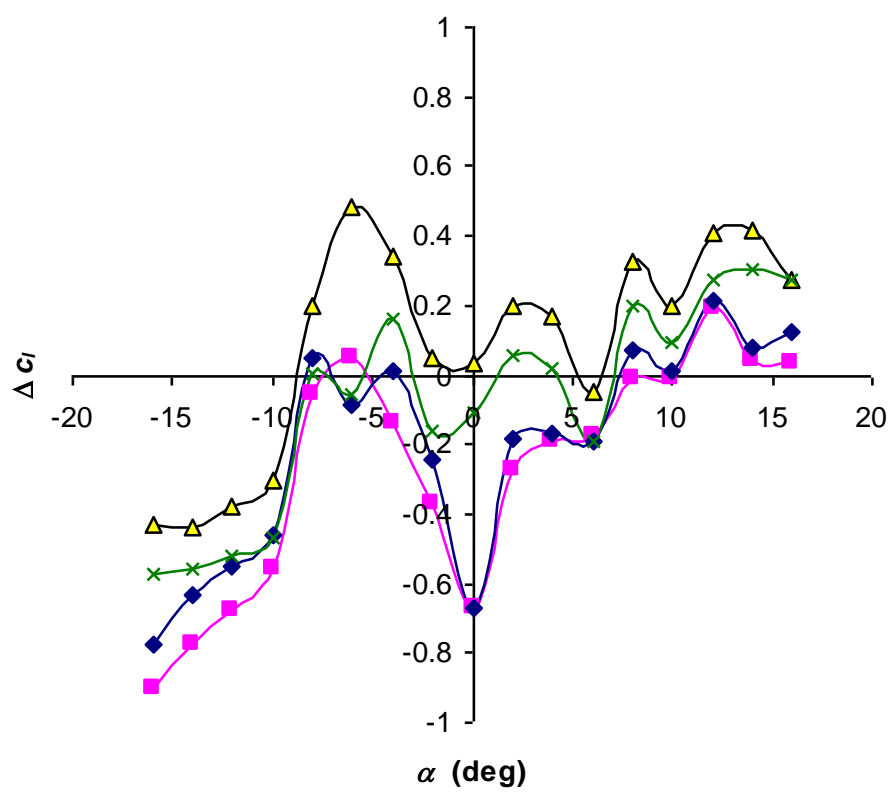

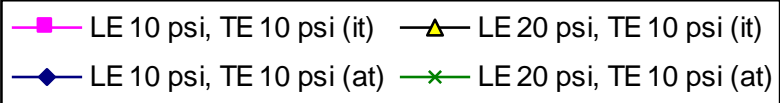

Figure B.39: Tunnel Velocity $=\mathbf{8 0} \mathrm{fps}$, Change in lift coefficient, Leading Edge Varying, Trailing Edge Blowing at 10 psi

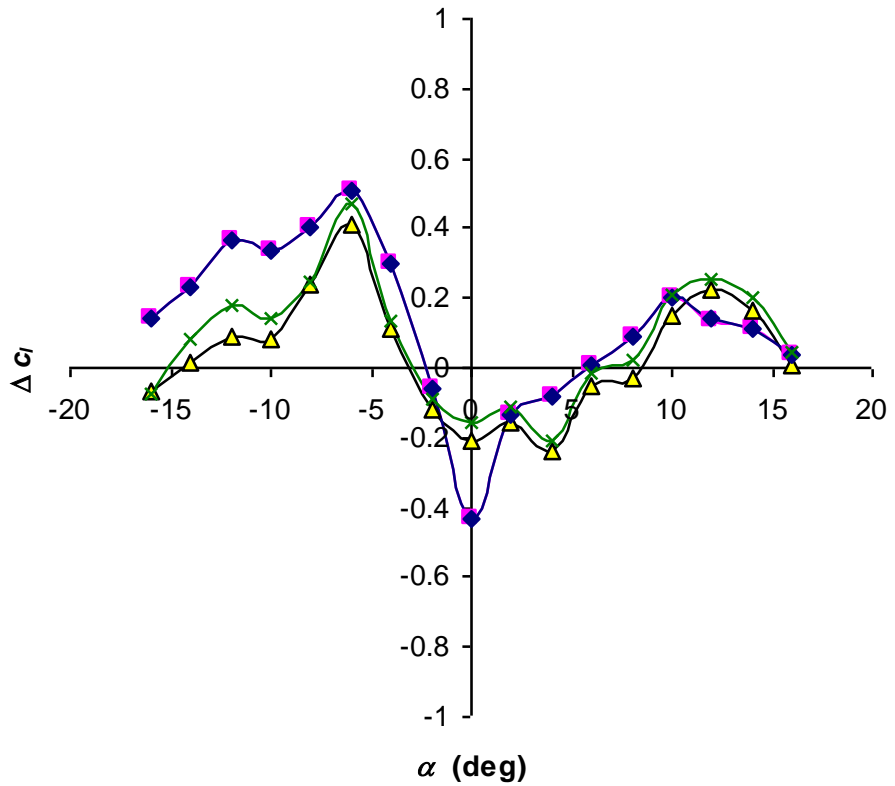

$\longrightarrow$ LE 10 psi, TE 10 psi (it) $\longleftarrow$ LE 20 psi, TE 10 psi (it)

$\multimap$ LE 10 psi, TE 10 psi (at) — LE 20 psi, TE 10 psi (at)

Figure B.40: Tunnel Velocity $=120 \mathrm{fps}$, Change in lift coefficient, Leading Edge Varying, Trailing Edge Blowing at 10 psi 


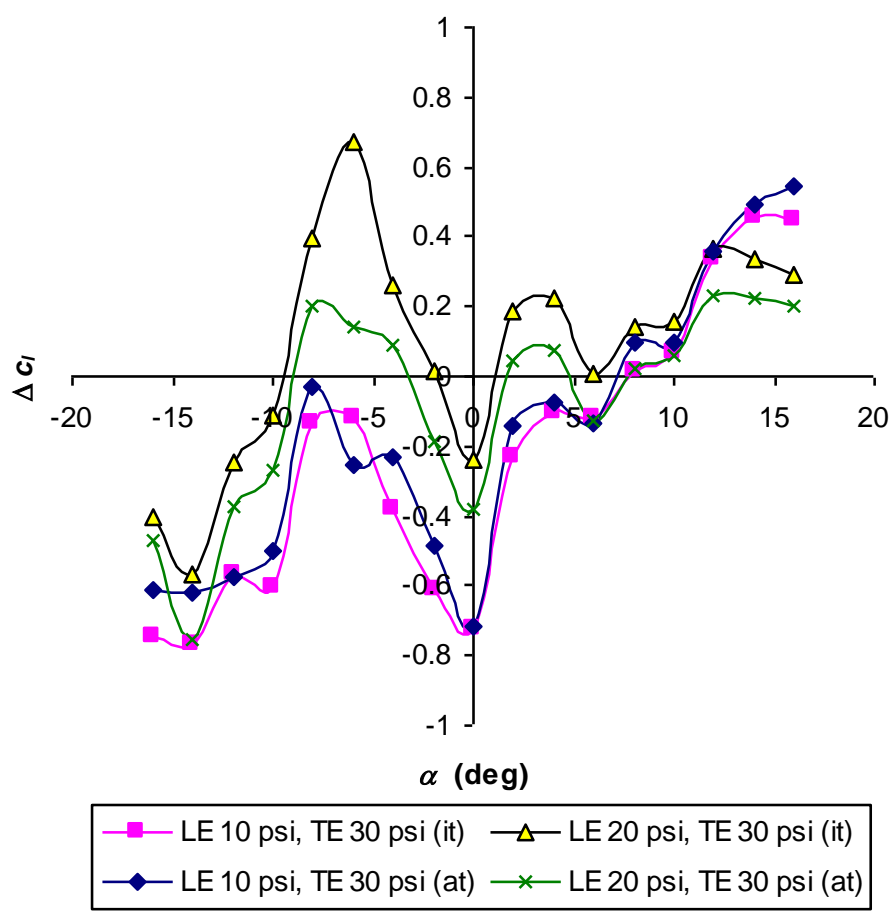

Figure B.41: Tunnel Velocity $=80 \mathrm{fps}$, Change in lift coefficient, Leading Edge Varying, Trailing Edge Blowing at 30 psi

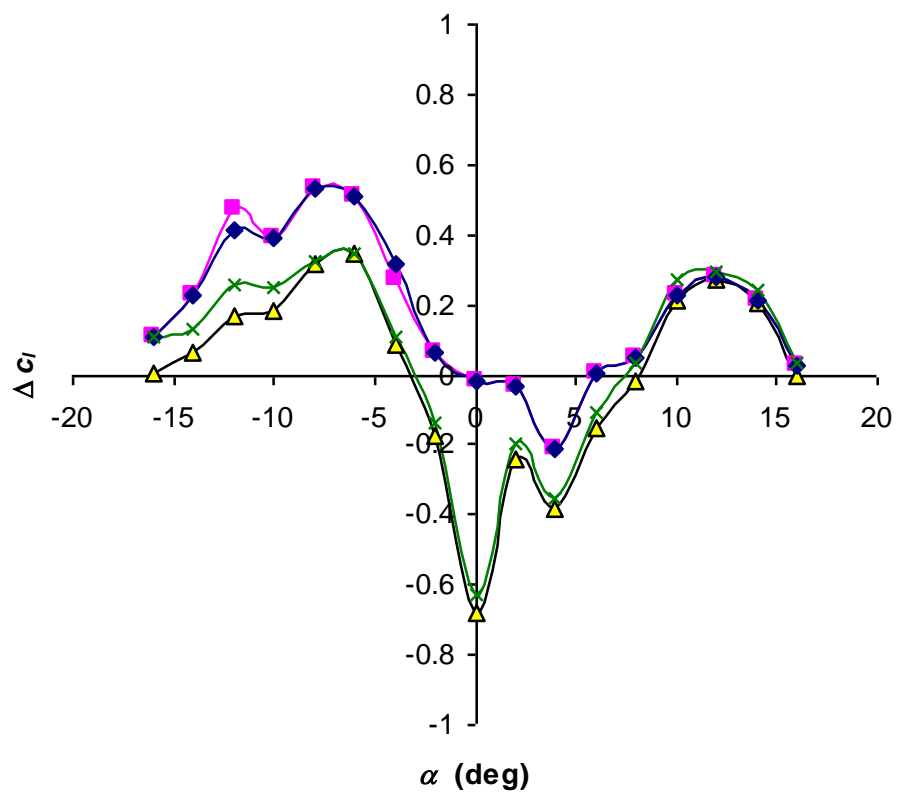

$\longrightarrow$ LE 10 psi, TE 30 psi (it) $\multimap$ LE20 psi, TE 30 psi (it)
$\longrightarrow$ LE 10 psi, TE 30 psi (at) $\longrightarrow$ LE20 psi, TE 30 psi (at)

Figure B.42: Tunnel Velocity $=120$ fps, Change in lift coefficient, Leading Edge Varying, Trailing Edge Blowing at $30 \mathrm{psi}$

\section{B.7 Lift Coefficient - Leading Edge Varying - Individual Tares Method}




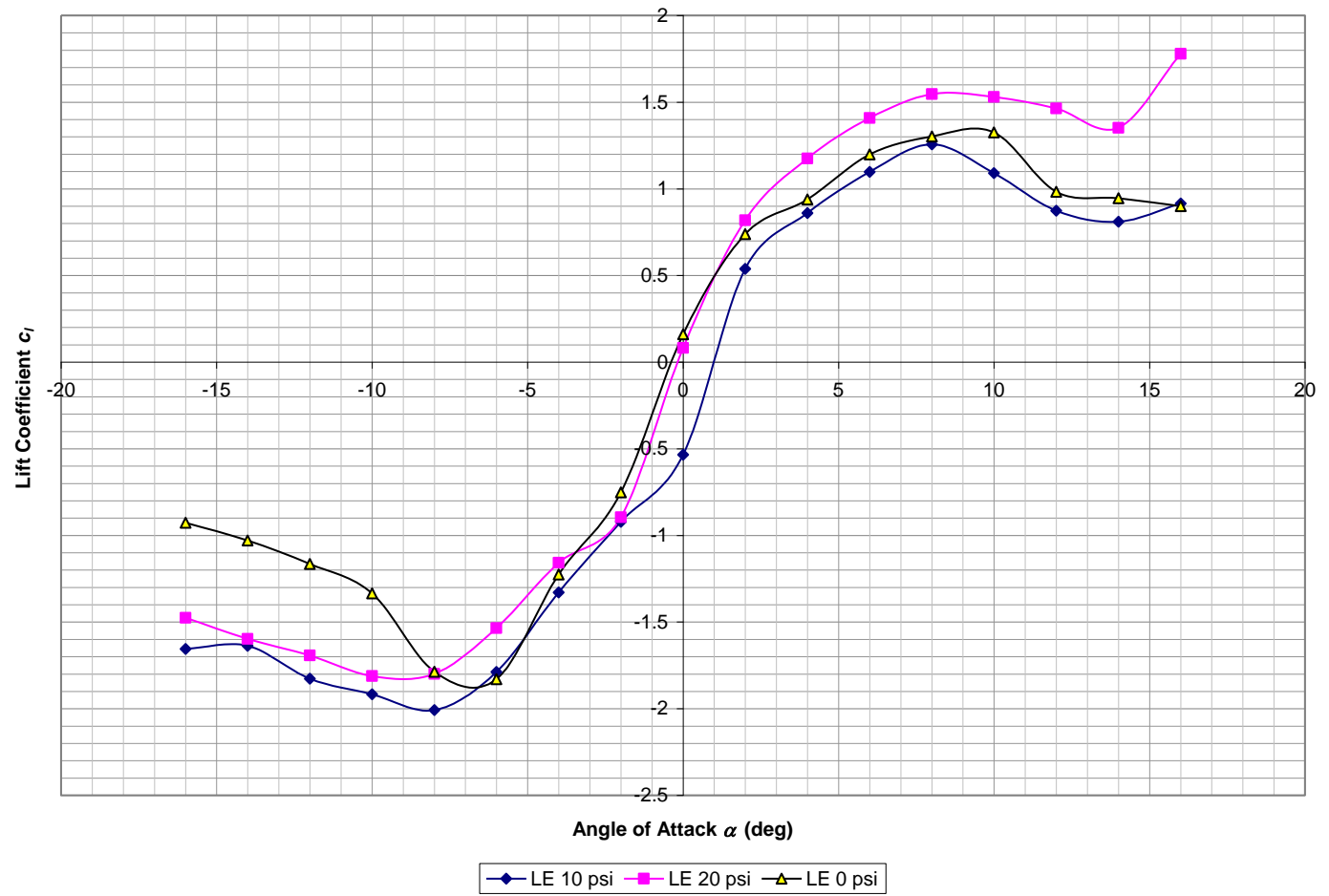

Figure B.43: Tunnel Velocity = 80 fps, Lift Coefficient vs. Angle of Attack, Leading Edge Blowing Only (individual tares)

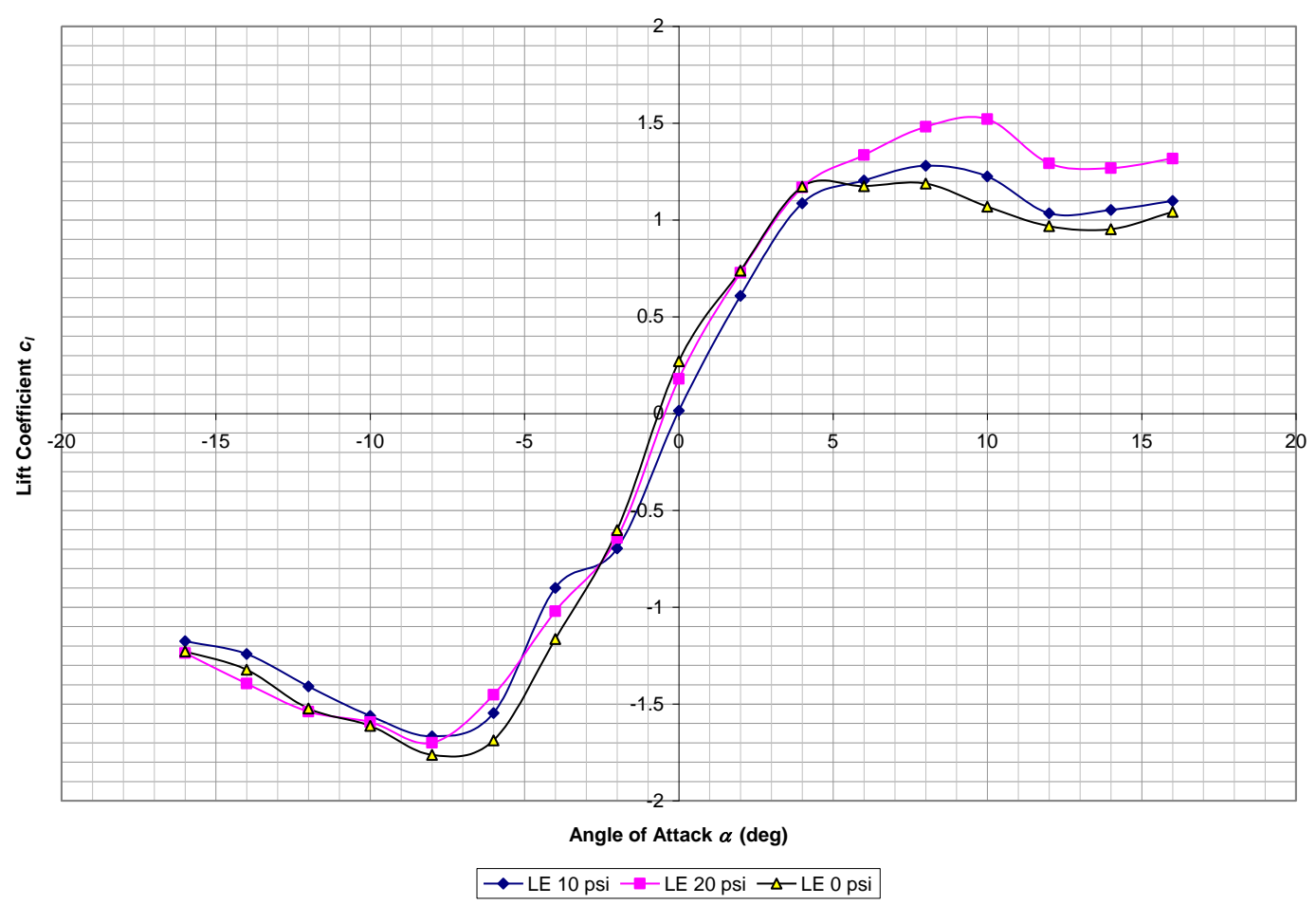

Figure B.44: Tunnel Velocity $=120$ fps, Lift Coefficient vs. Angle of Attack, Leading Edge Blowing Only (individual tares) 


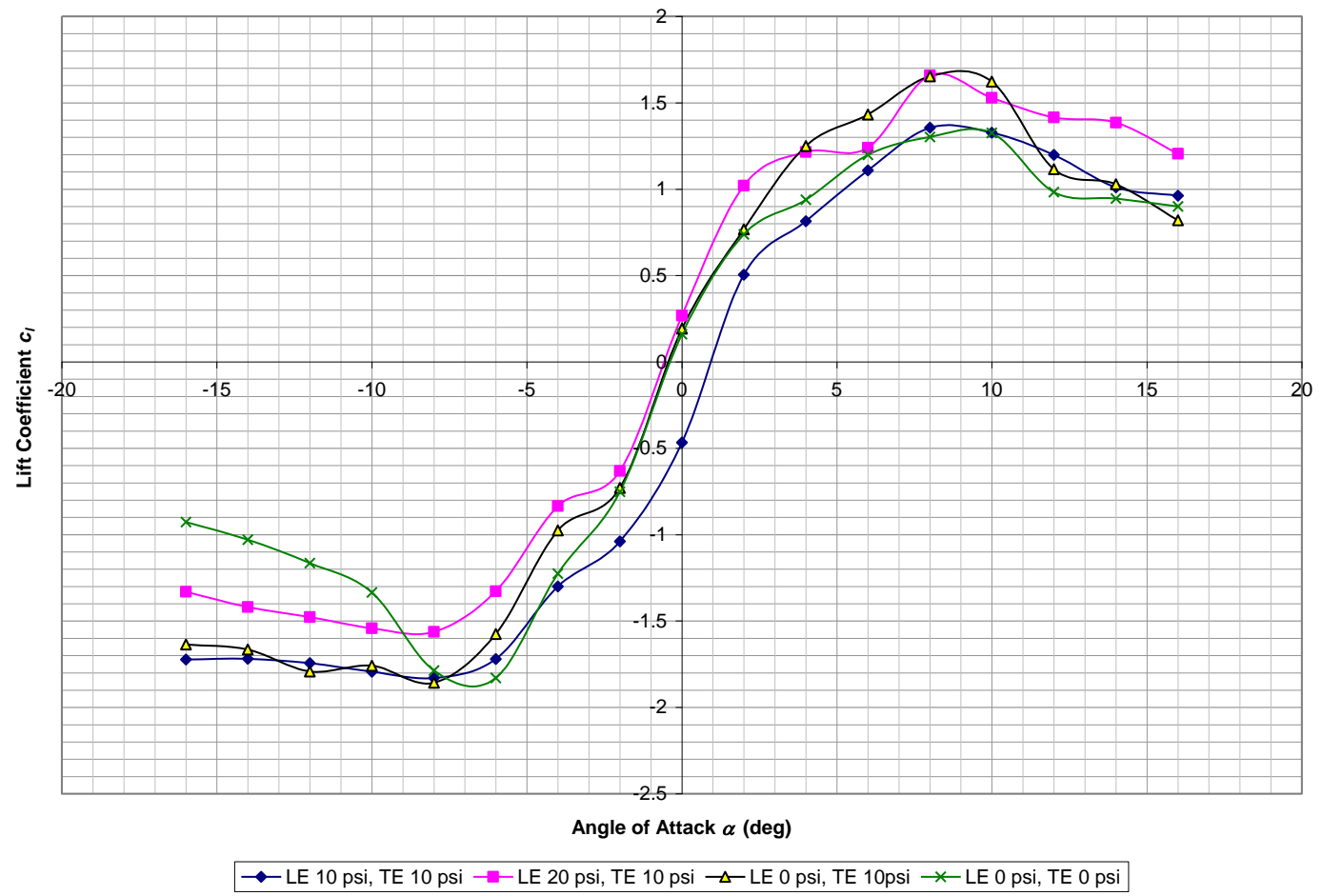

Figure B.45: Tunnel Velocity $=80$ fps, Lift Coefficient vs. Angle of Attack, Leading Edge Varying, Trailing Blowing at 10 psi (individual tares)

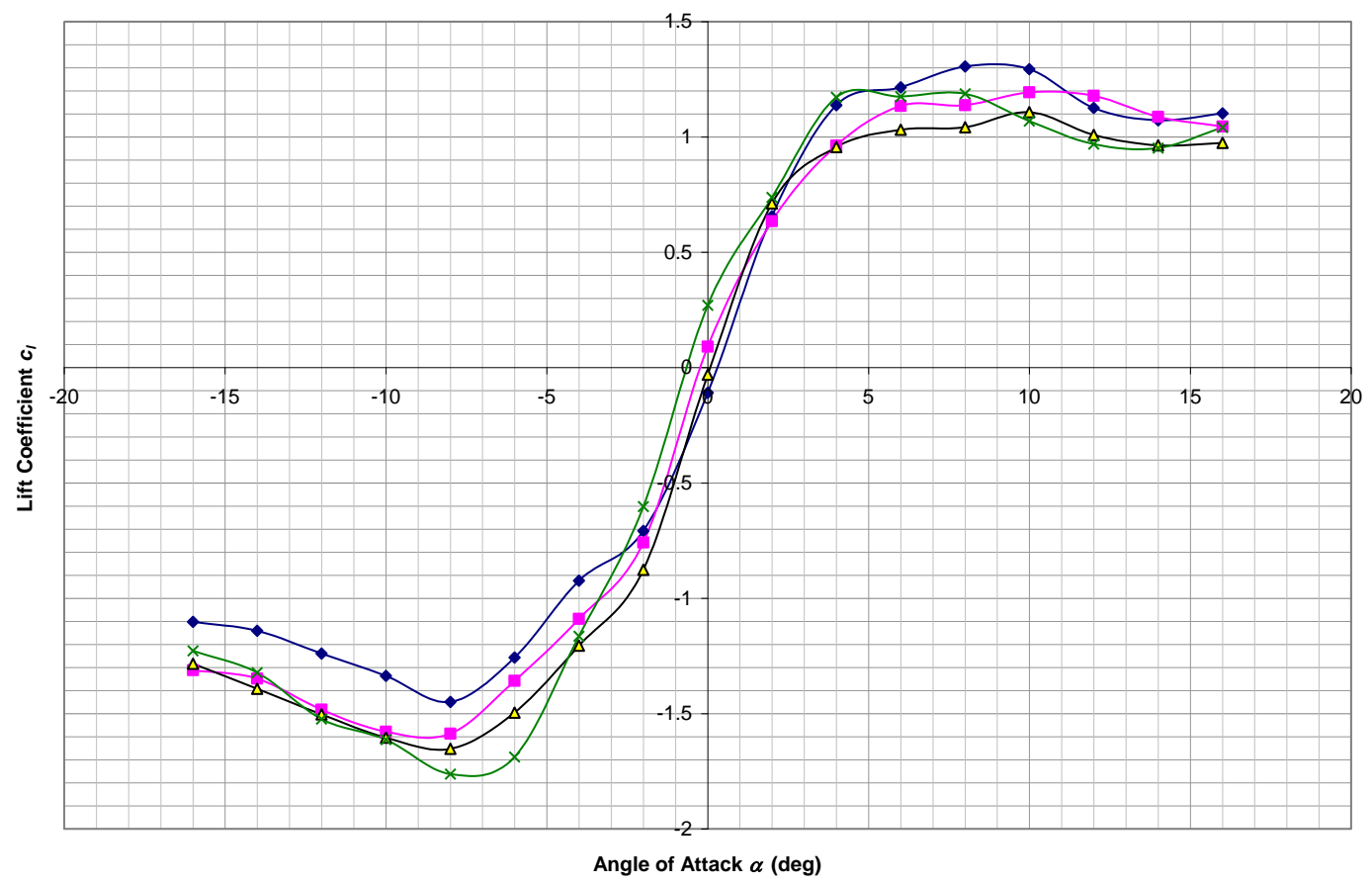

$\rightarrow-$ LE 10 psi, TE 10 psi $\rightarrow$ LE 20 psi, TE 10 psi $\rightarrow-$ LE 0 psi, TE 10psi $\rightarrow$ LE 0 psi, TE 0 psi

Figure B.46: Tunnel Velocity = 120 fps, Lift Coefficient vs. Angle of Attack, Leading Edge Varying, Trailing Blowing at 10 psi (individual tares) 


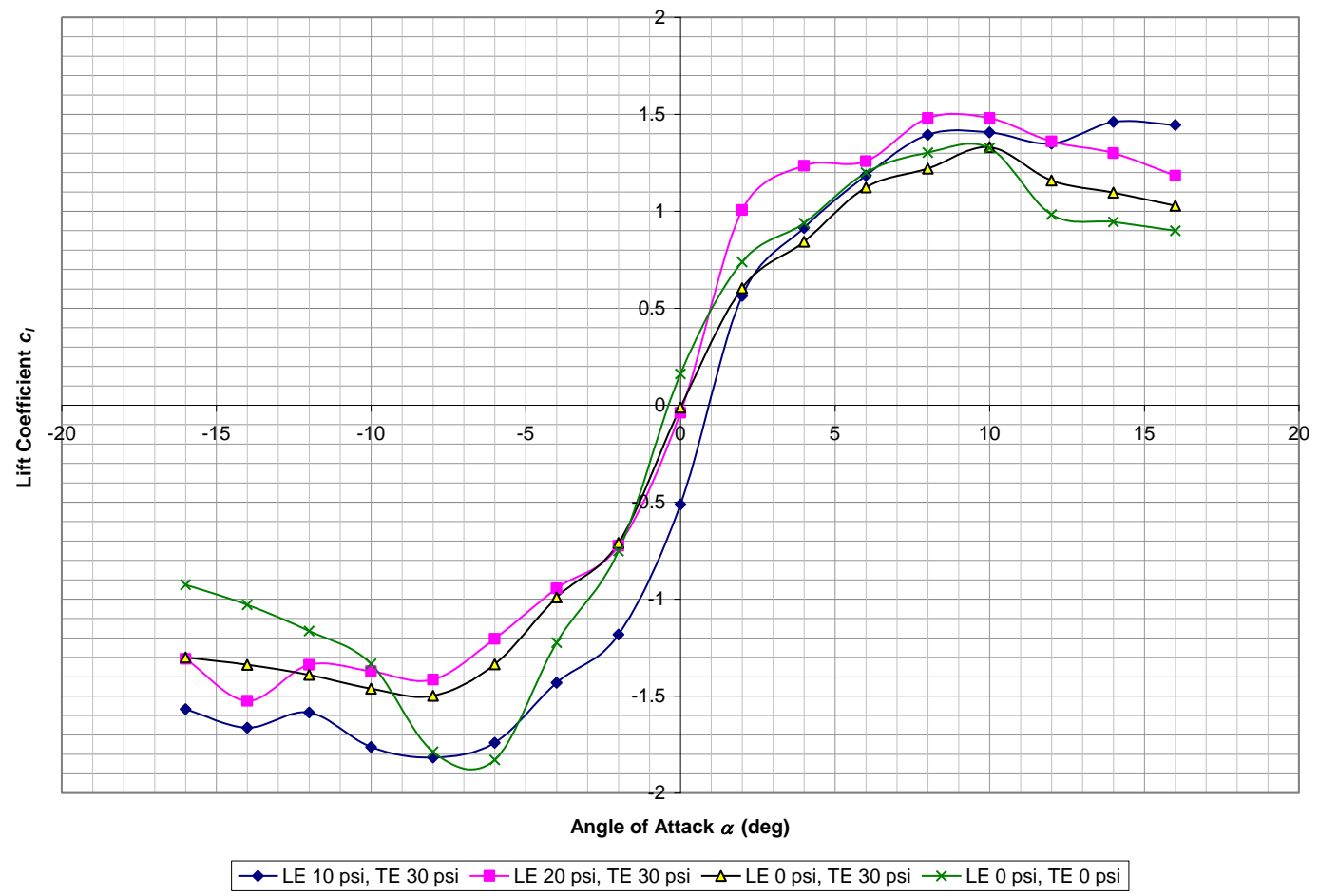

Figure B.47: Tunnel Velocity $=80$ fps, Lift Coefficient vs. Angle of Attack, Leading Edge Varying, Trailing Blowing at 30 psi (individual tares)

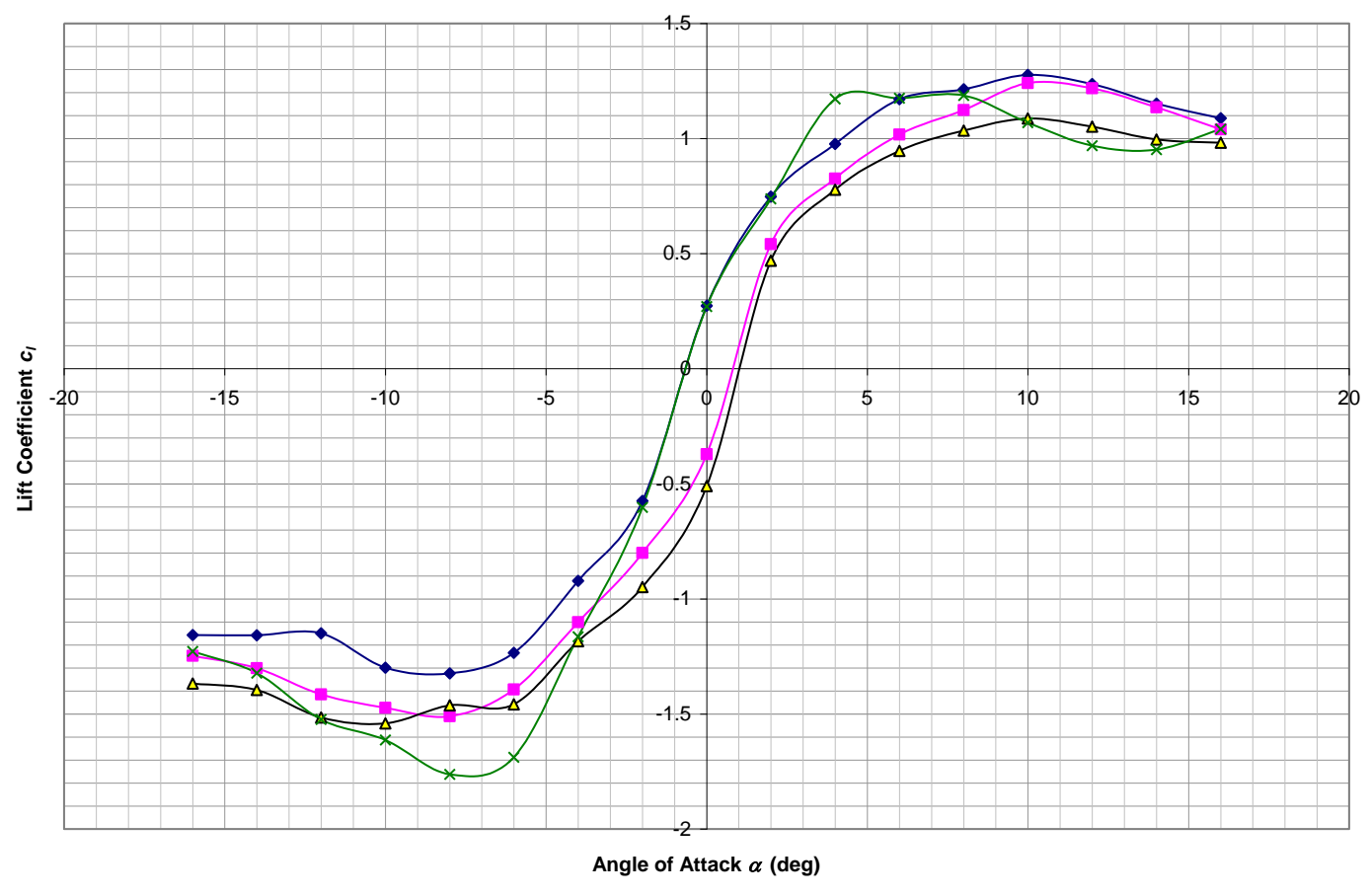

$\rightarrow-$ LE 10 psi, TE 30 psi $\rightarrow$ LE 20 psi, TE 30 psi $\rightarrow-$ LE 0 psi, TE 30 psi $*$ LE 0 psi, TE 0 psi

Figure B.48: Tunnel Velocity = 120 fps, Lift Coefficient vs. Angle of Attack, Leading Edge Varying, Trailing Blowing at 30 psi (individual tares)

\section{B.8 Drag Coefficient - Trailing Edge Varying - Average Tare Method}




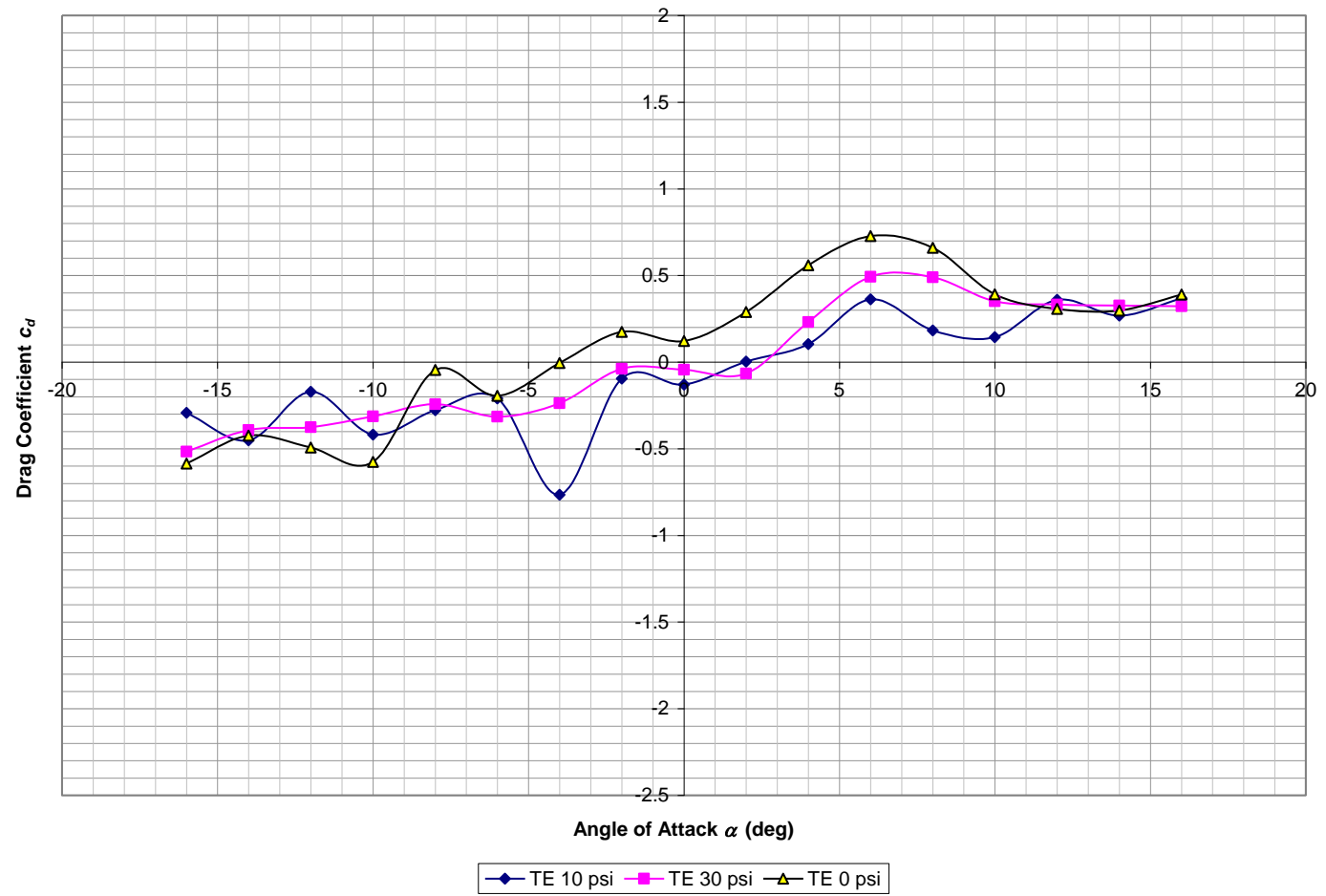

Figure B.49: Tunnel Velocity = 80 fps, Drag Coefficient vs. Angle of Attack, Trailing Edge Blowing Only (average tare)

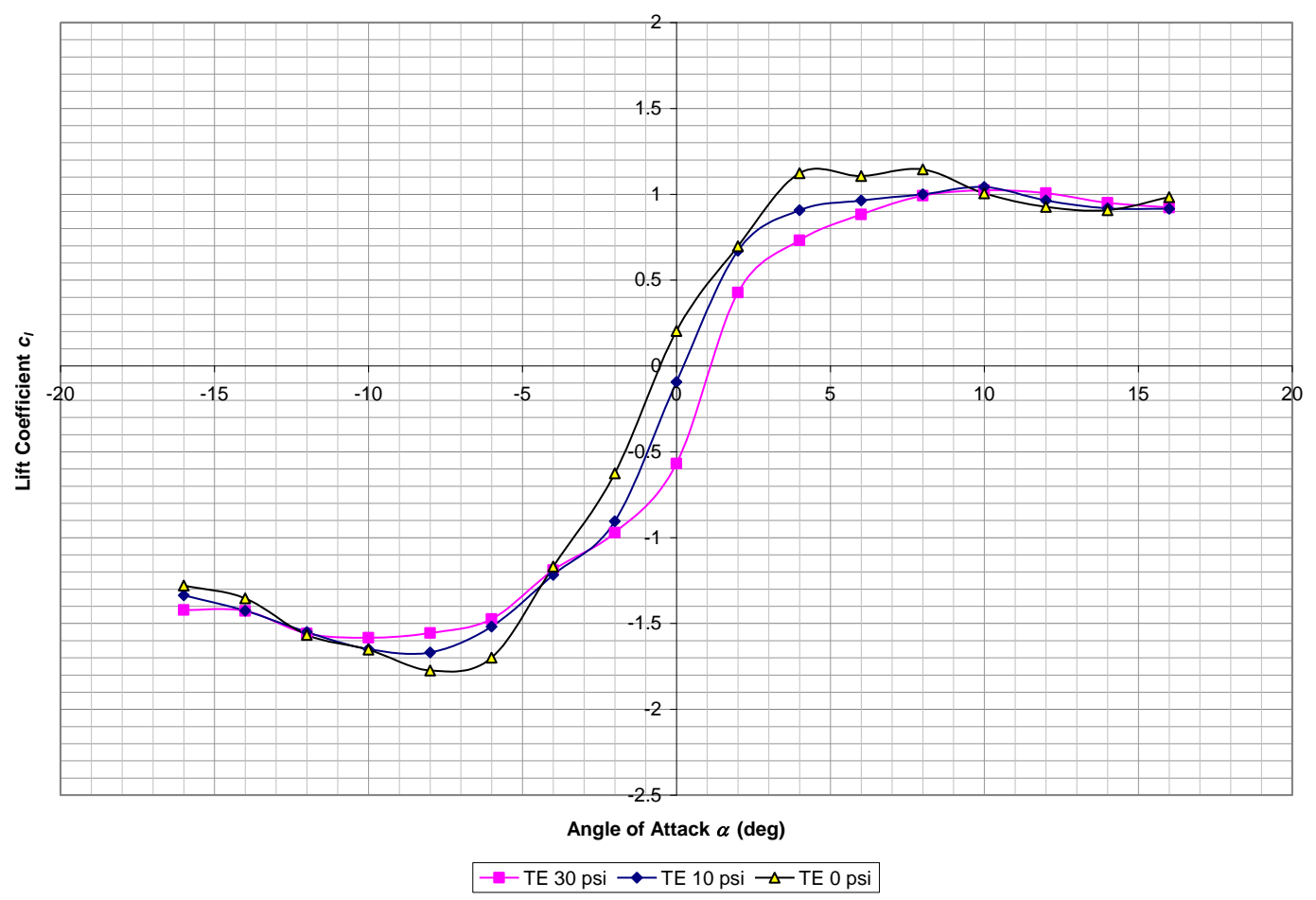

Figure B.50: Tunnel Velocity = 120 fps, Drag Coefficient vs. Angle of Attack, Trailing Edge Blowing Only (average tare) 


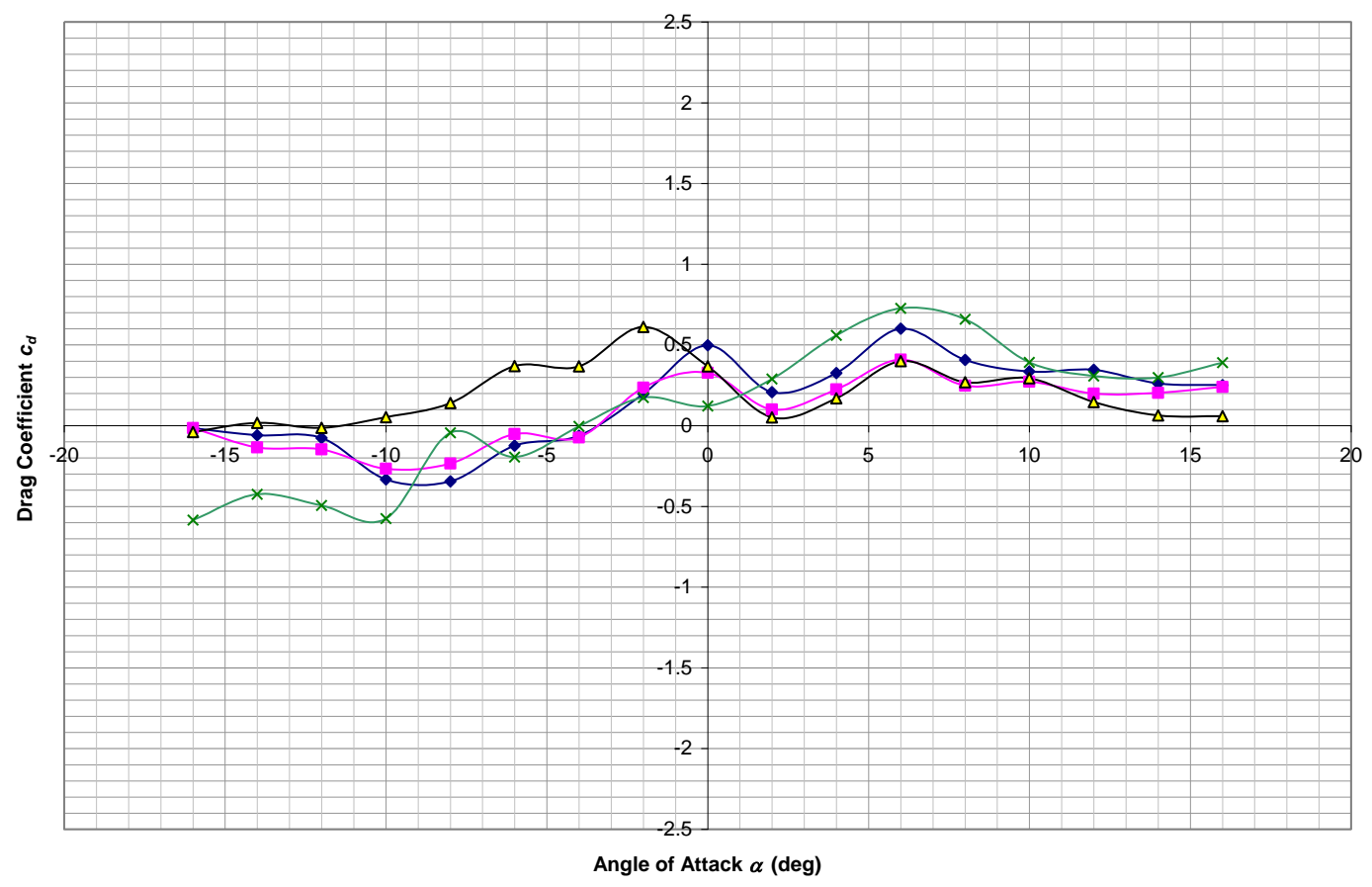

$\multimap-$ LE 10 psi, TE 0 psi $\rightarrow$ LE 10 psi, TE 10 psi $\neg-$ LE 10 psi, TE 30 psi $\rightarrow$ LE 0 psi, TE 0 psi

Figure B.51: Tunnel Velocity = 80 fps, Drag Coefficient vs. Angle of Attack, Trailing Edge Varying, Leading Edge Blowing at $10 \mathrm{psi}$ (average tare)

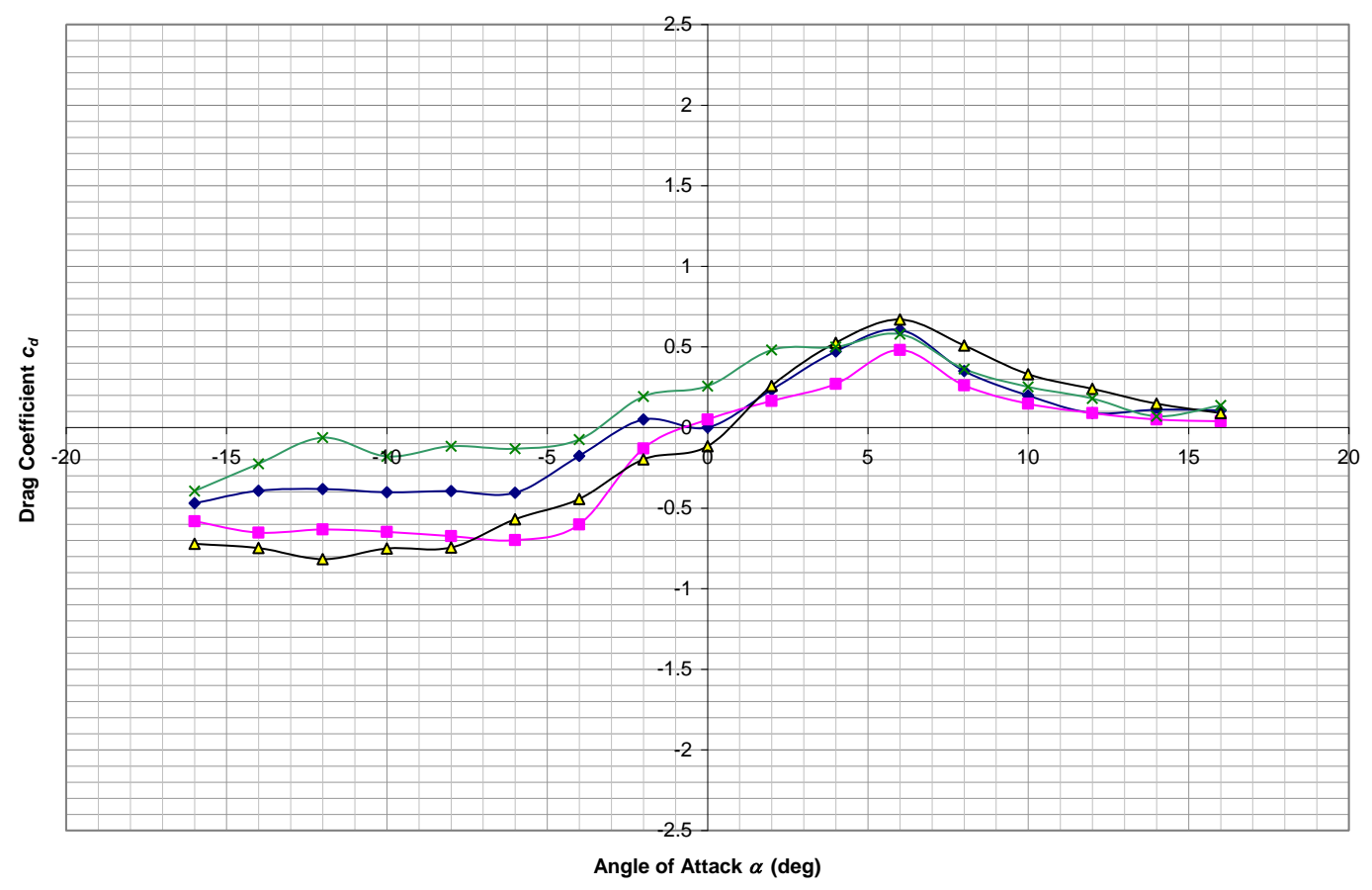

$\rightarrow-$ LE 10 psi, TE 0 psi $\rightarrow$ LE 10 psi, TE 10 psi $\rightarrow-$ LE 10 psi, TE 30 psi $\rightarrow$ LE 0 psi, TE 0 psi

Figure B.52: Tunnel Velocity = 120 fps, Drag Coefficient vs. Angle of Attack, Trailing Edge Varying, Leading Edge Blowing at $10 \mathrm{psi}$ (average tare) 


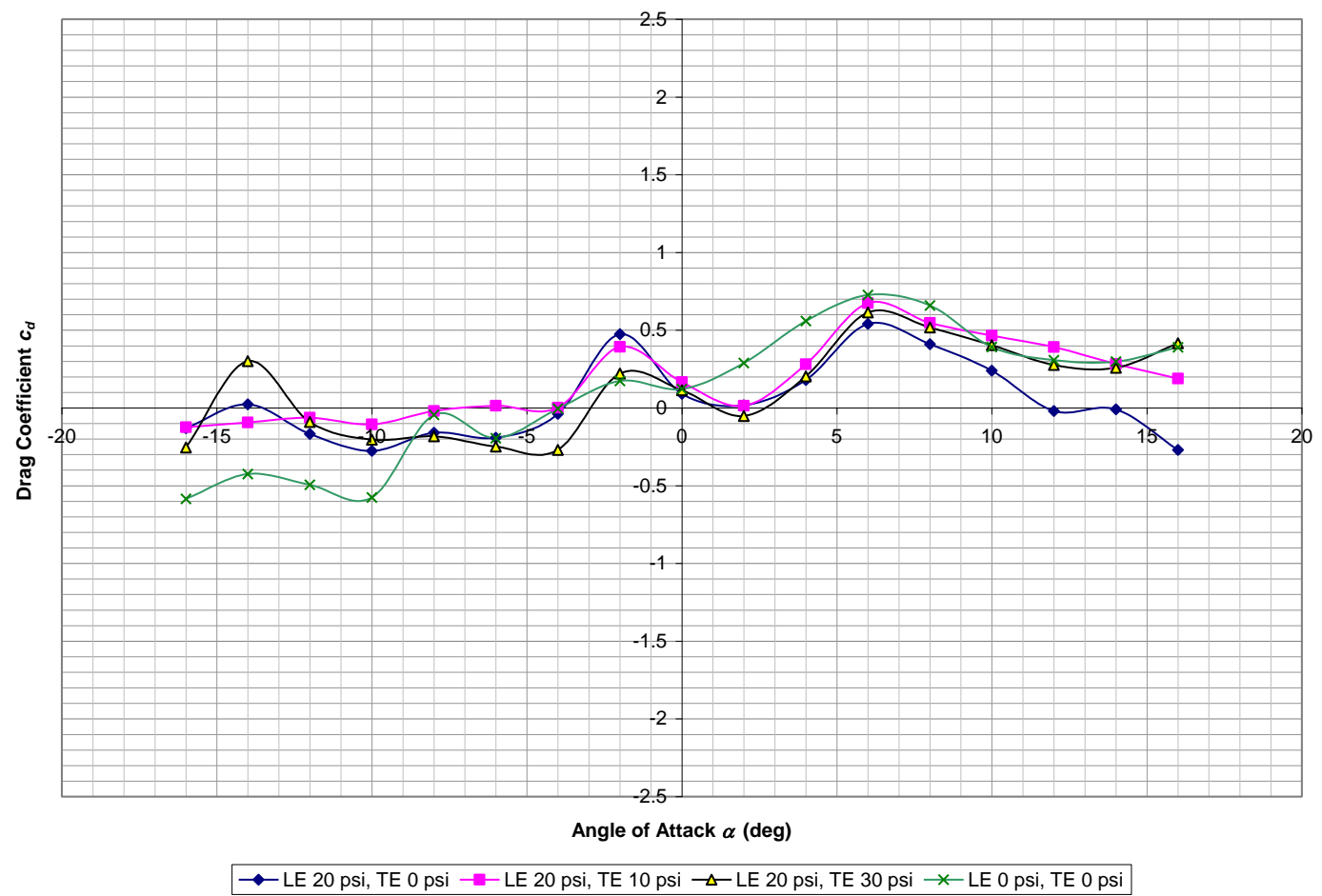

Figure B.53: Tunnel Velocity = 80 fps, Drag Coefficient vs. Angle of Attack, Trailing Edge Varying, Leading Edge Blowing at 20 psi (average tare)

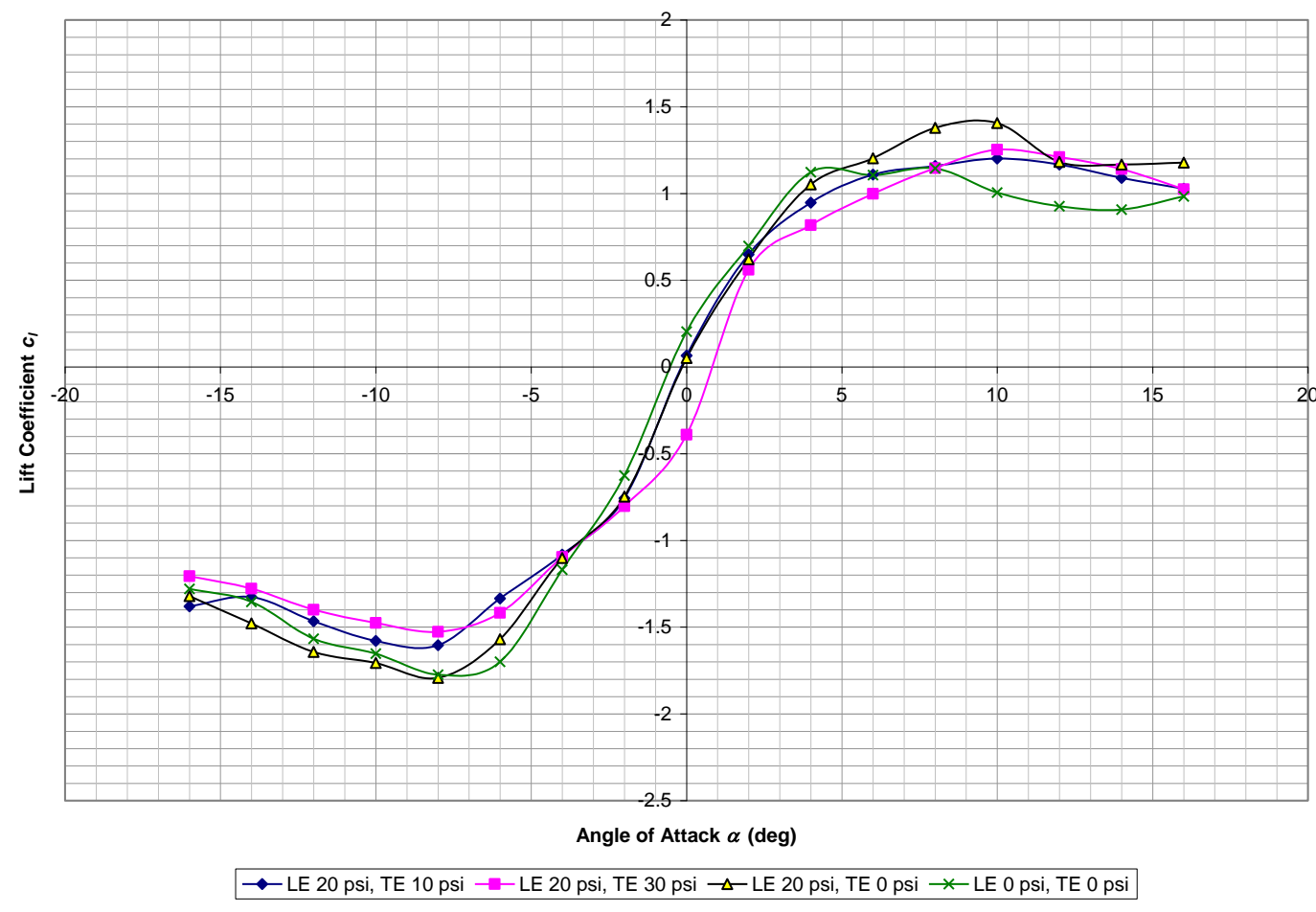

Figure B.54: Tunnel Velocity = $120 \mathrm{fps,} \mathrm{Drag} \mathrm{Coefficient} \mathrm{vs.} \mathrm{Angle} \mathrm{of} \mathrm{Attack,} \mathrm{Trailing} \mathrm{Edge} \mathrm{Varying,} \mathrm{Leading}$ Edge Blowing at 20 psi (average tare)

\section{B.9 Drag Coefficient - Trailing Edge Varying - Individual Tares Method}




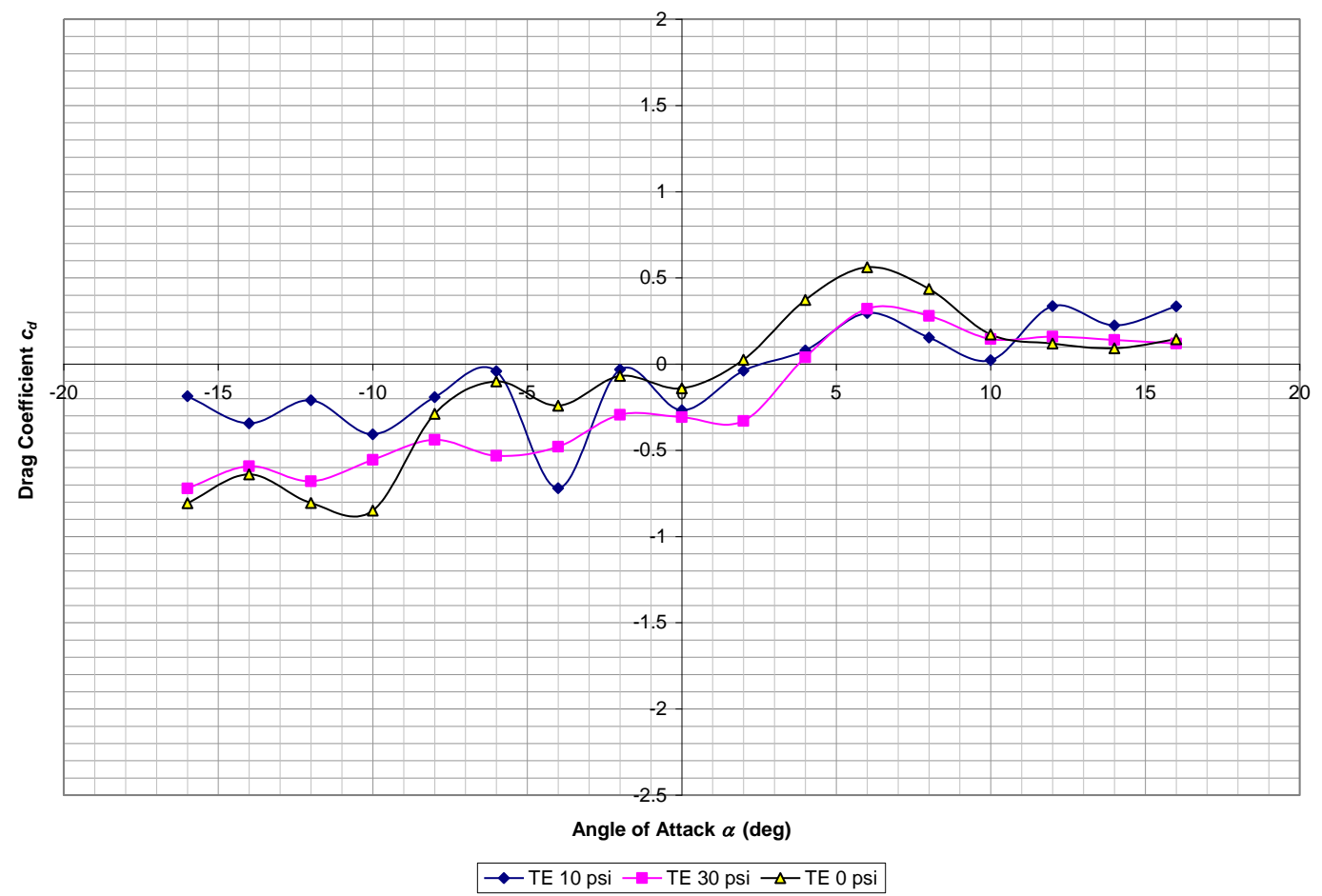

Figure B.55: Tunnel Velocity = 80 fps, Drag Coefficient vs. Angle of Attack, Trailing Edge Blowing Only (individual tares)

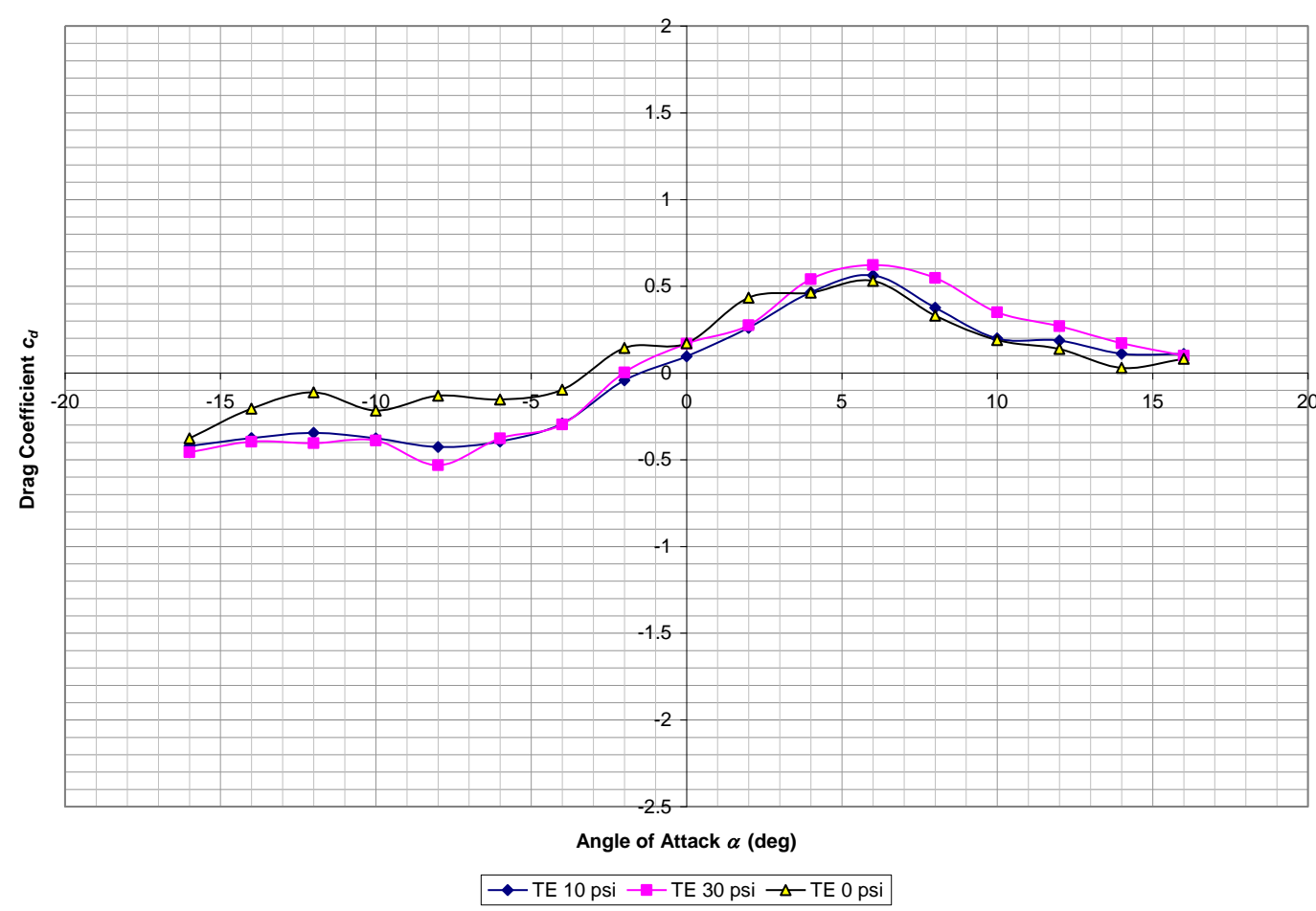

Figure B.56: Tunnel Velocity = 120 fps, Drag Coefficient vs. Angle of Attack, Trailing Edge Blowing Only (individual tares) 


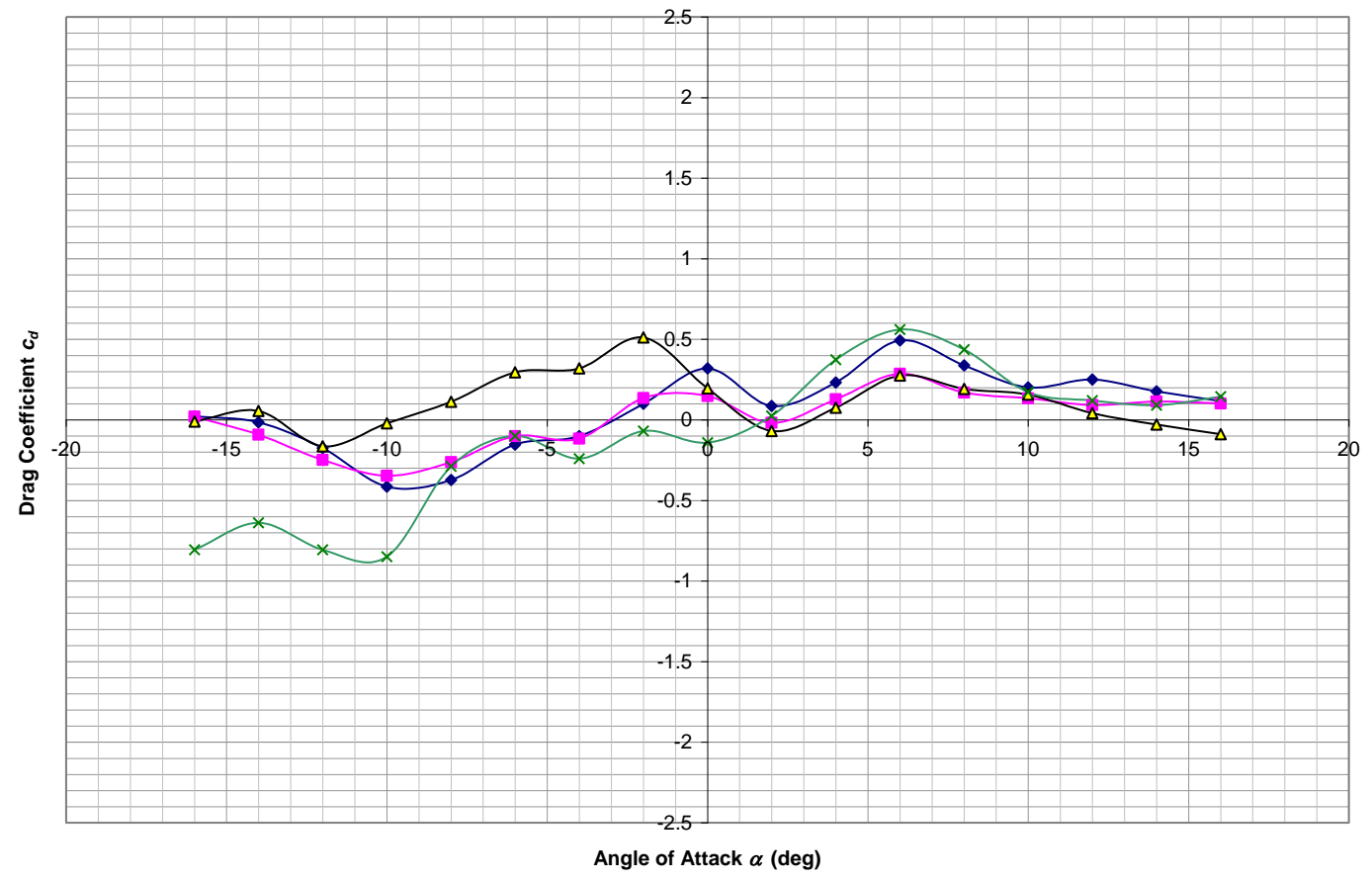

$\rightarrow-$ LE 10 psi, TE 0 psi $\rightarrow$ LE 10 psi, TE 10 psi $\multimap-$ LE 10 psi, TE 30 psi $\rightarrow$ LE 0 psi, TE 0 psi

Figure B.57: Tunnel Velocity $=80 \mathrm{fps}$, Drag Coefficient vs. Angle of Attack, Trailing Edge Varying, Leading Edge Blowing at $10 \mathrm{psi}$ (individual tares)

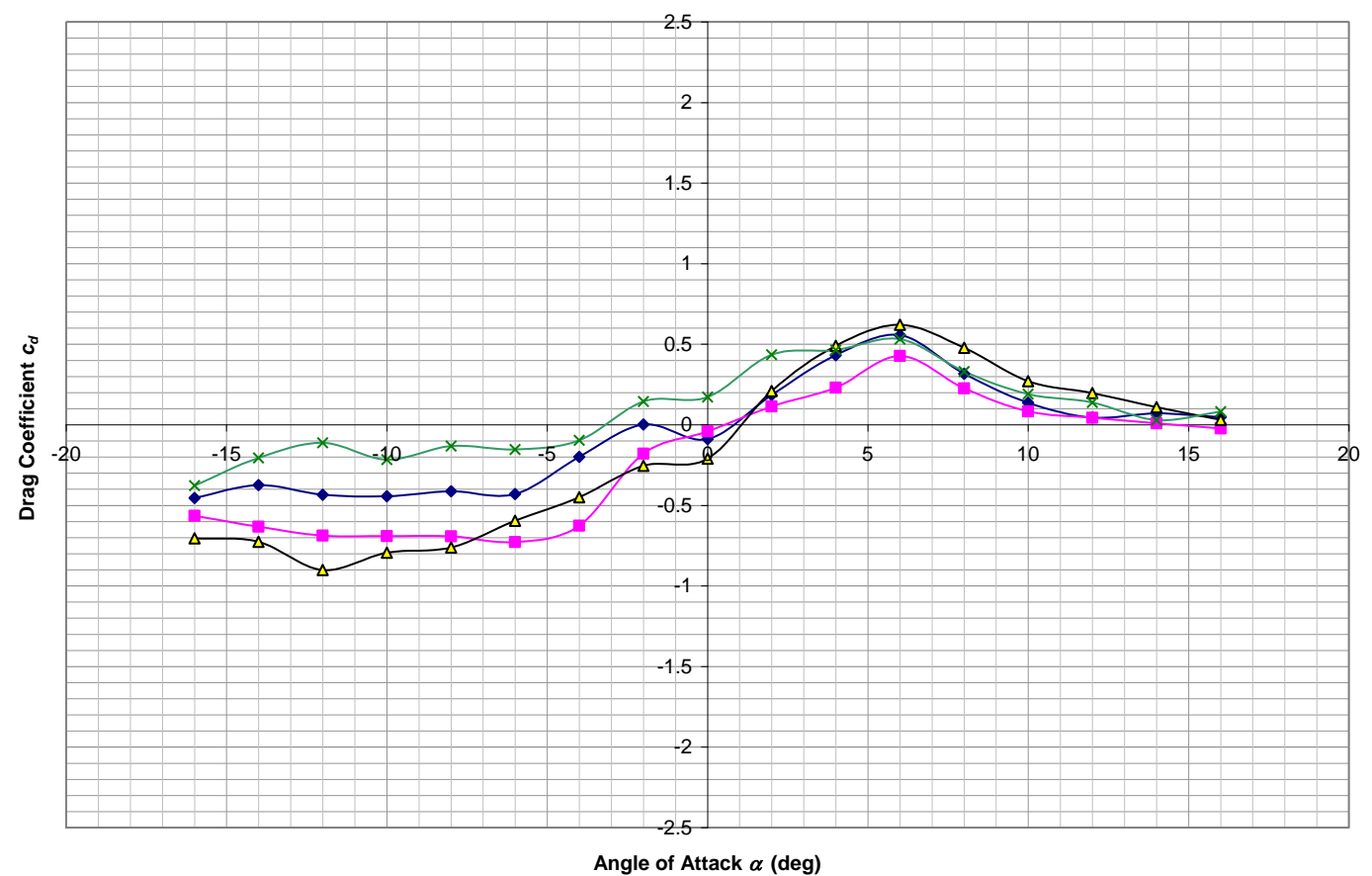

$\rightarrow-$ LE 10 psi, TE 0 psi $\rightarrow$ LE 10 psi, TE 10 psi $\rightarrow-$ LE 10 psi, TE 30 psi $¥$ LE 0 psi, TE 0 psi

Figure B.58: Tunnel Velocity = 120 fps, Drag Coefficient vs. Angle of Attack, Trailing Edge Varying, Leading Edge Blowing at 10 psi (individual tares) 


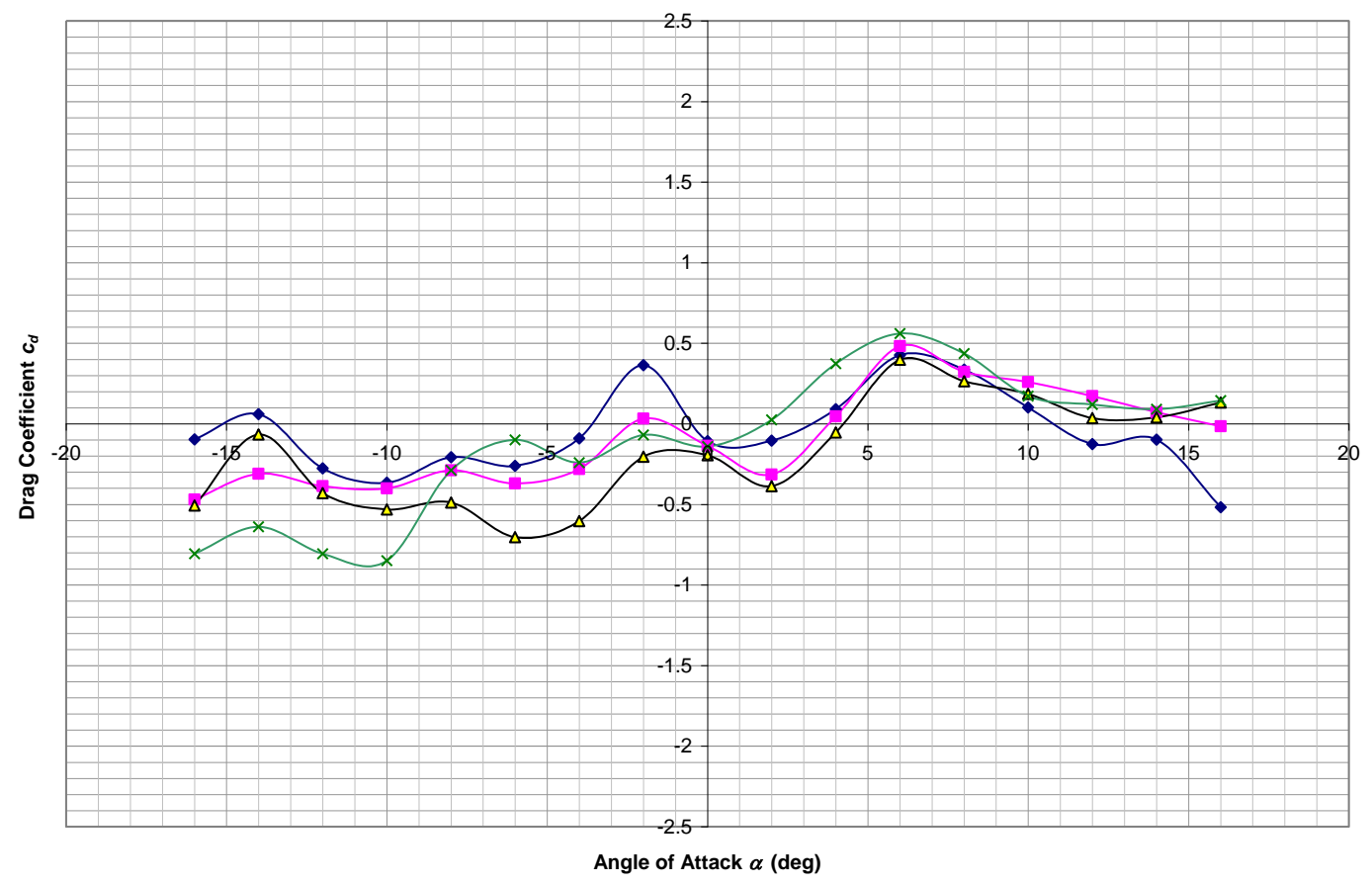

$\rightarrow-$ LE 20 psi, TE 0 psi $\rightarrow-$ LE 20 psi, TE 10 psi $\rightarrow-$ LE 20 psi, TE 30 psi $\rightarrow$ LE 0 psi, TE 0 psi

Figure B.59: Tunnel Velocity = 80 fps, Drag Coefficient vs. Angle of Attack, Trailing Edge Varying, Leading Edge Blowing at 20 psi (individual tares)

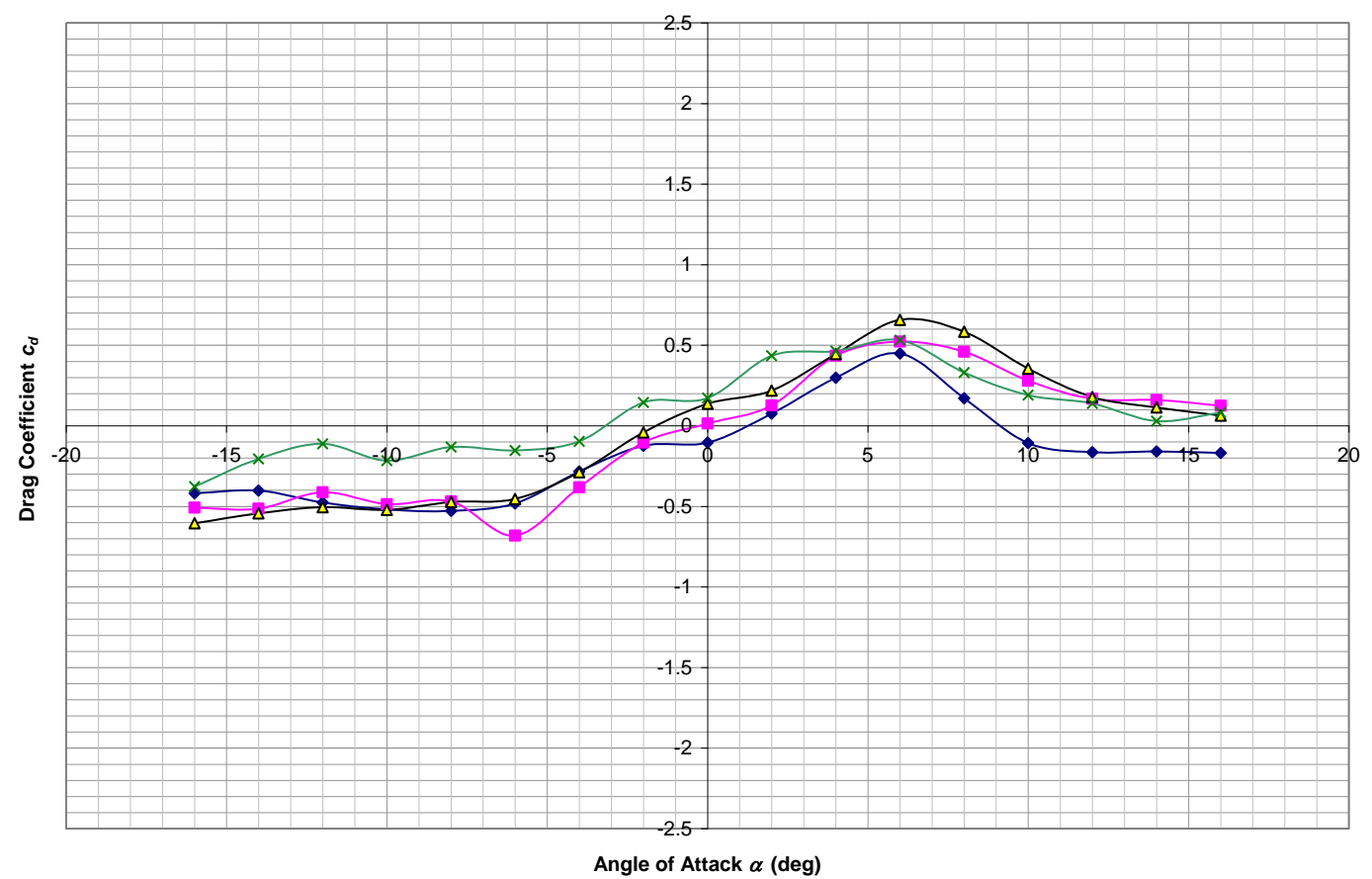

$\rightarrow-$ LE 20 psi, TE 0 psi $\rightarrow$ LE 20 psi, TE 10 psi $\rightarrow-$ LE 20 psi, TE 30 psi $\rightarrow$ LE 0 psi, TE 0 psi

Figure B.60: Tunnel Velocity = 120 fps, Drag Coefficient vs. Angle of Attack, Trailing Edge Varying, Leading Edge Blowing at 20 psi (individual tares)

\section{B.10 Drag Coefficient Change - Trailing Edge Varying}




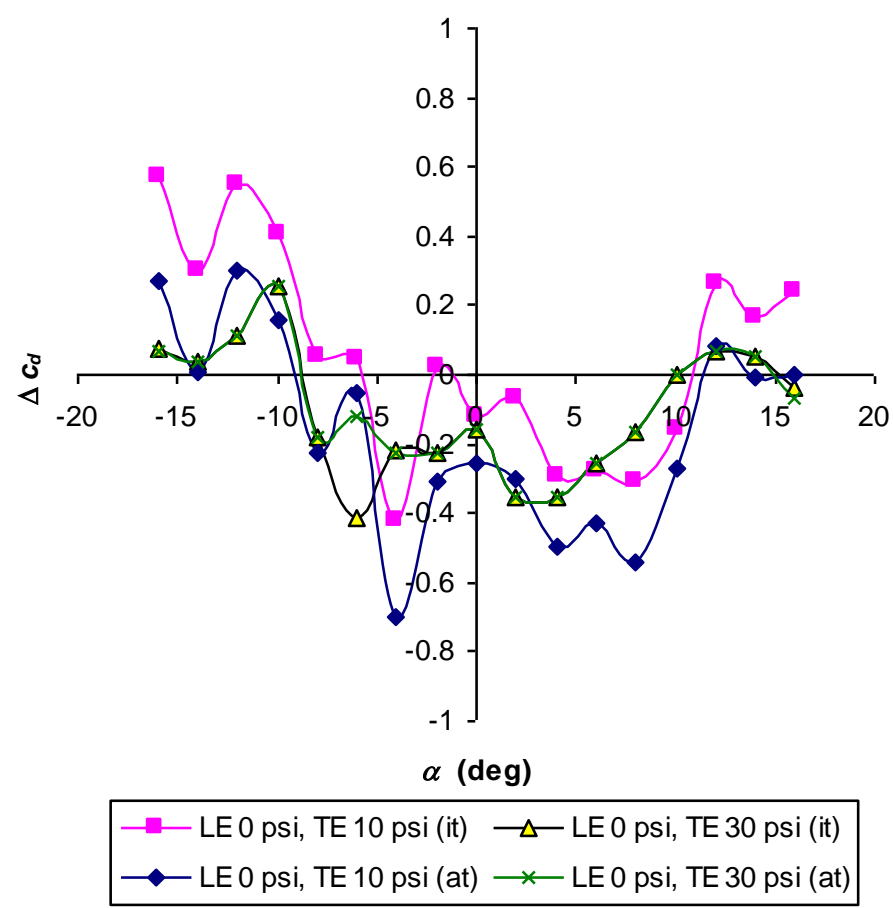

Figure B.61: Tunnel Velocity $=\mathbf{8 0}$ fps, Drag Coefficient Change vs. Angle of Attack, Trailing Edge Blowing Only

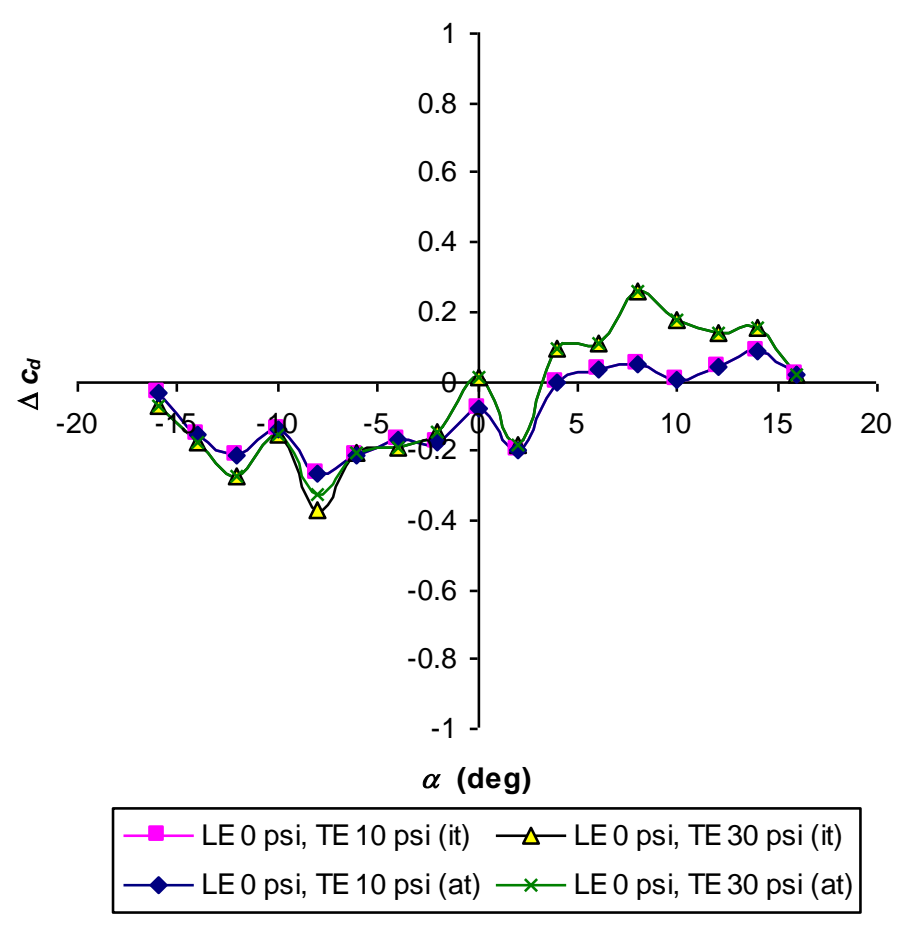

Figure B.62: Tunnel Velocity = 120 fps, Drag Coefficient Change vs. Angle of Attack, Trailing Edge Blowing Only 


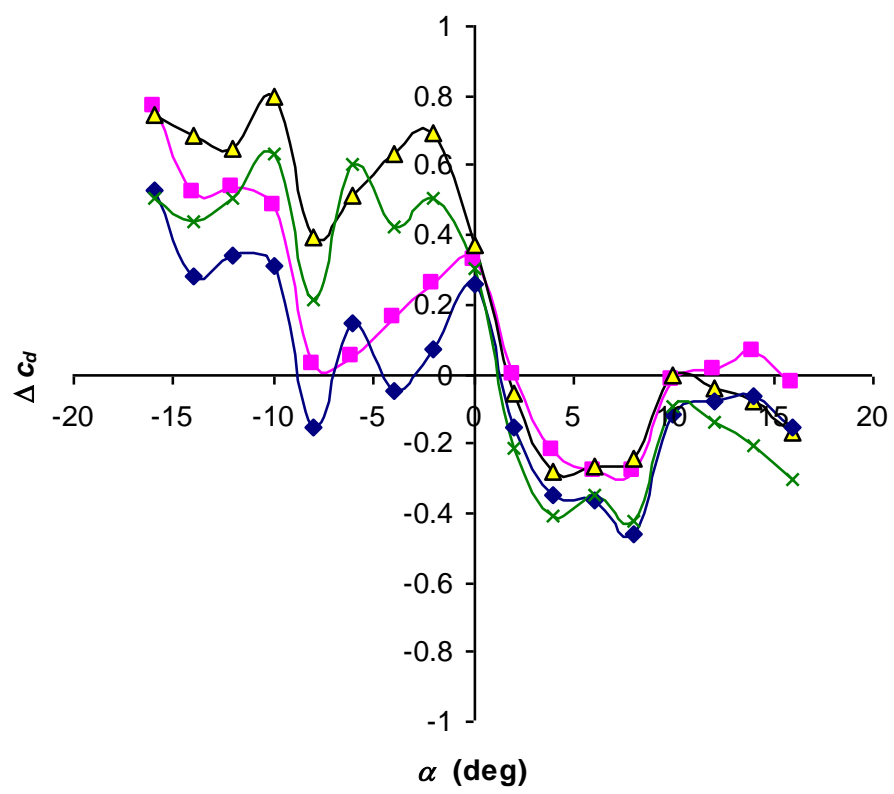

- LE 10 psi, TE 10 psi (it) $\multimap-$ LE 10 psi, TE 30 psi (it)

$\multimap$ LE 10 psi, TE 10 psi (at) $\multimap$ LE 10 psi, TE 30 psi (at)

Figure B.63: Tunnel Velocity = 80 fps, Drag Coefficient Change vs. Angle of Attack, Trailing Edge Varying, Leading Edge Blowing at 10 psi

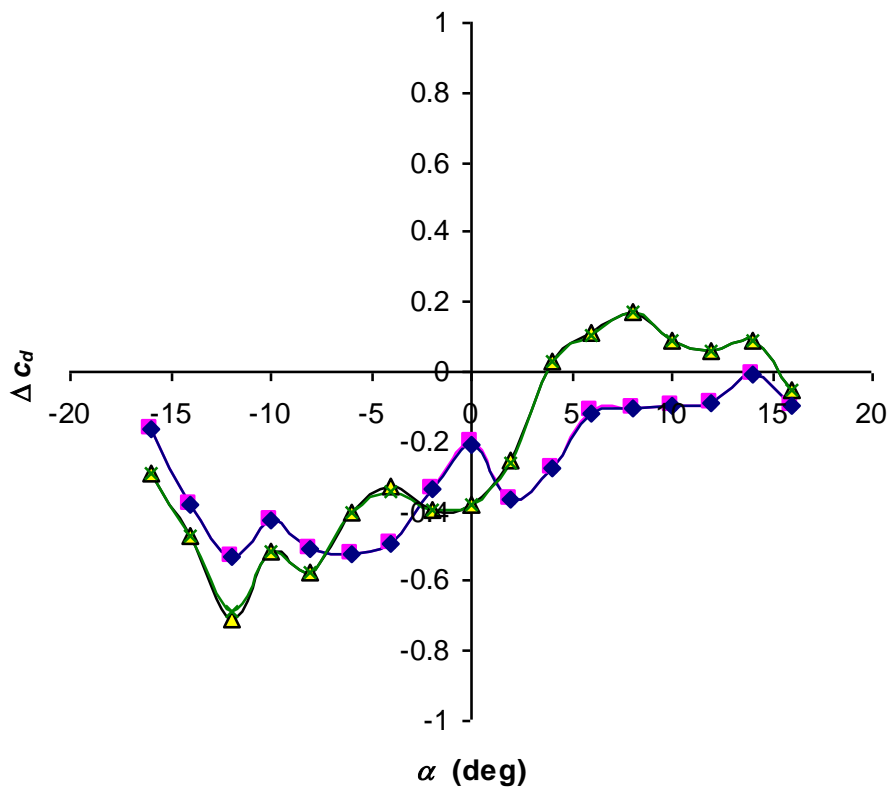

$\longrightarrow$ LE 10 psi, TE 10 psi (it) $\longrightarrow$ LE 10 psi, TE 30 psi (it)

$\multimap$ LE 10 psi, TE 10 psi (at) $\multimap$ LE 10 psi, TE 30 psi (at)

Figure B.64: Tunnel Velocity = 120 fps, Drag Coefficient Change vs. Angle of Attack, Trailing Edge Varying, Leading Edge Blowing at 10 psi 


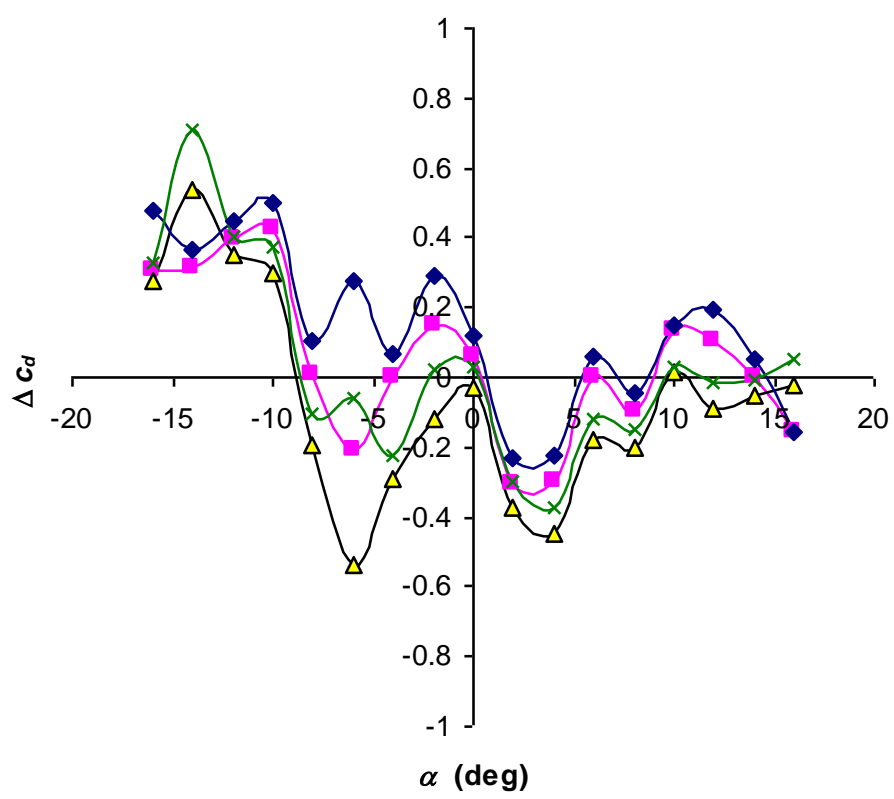

- LE 20 psi, TE 10 psi (it) $\triangle-$ LE 20 psi, TE 30 psi (it)

$\multimap$ LE 20 psi, TE 10 psi (at) $¥$ LE 10 psi, TE 30 psi (at)

Figure B.65: Tunnel Velocity = 80 fps, Drag Coefficient Change vs. Angle of Attack, Trailing Edge Varying, Leading Edge Blowing at 20 psi

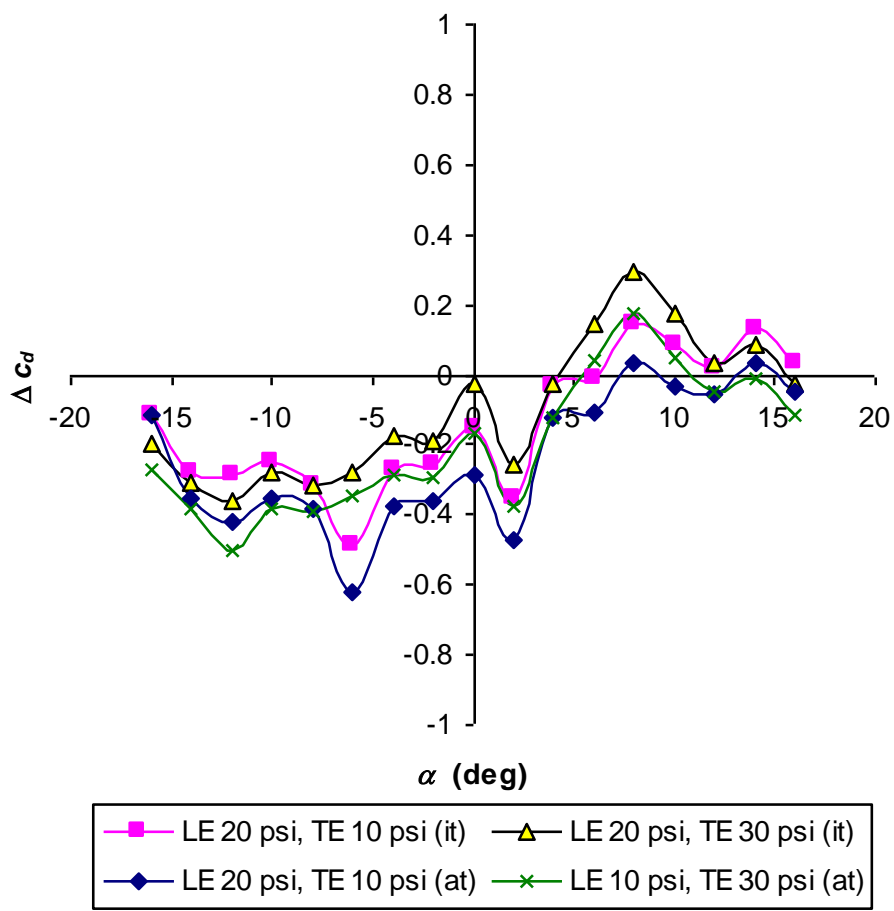

Figure B.66: Tunnel Velocity = 120 fps, Drag Coefficient Change vs. Angle of Attack, Trailing Edge Varying, Leading Edge Blowing at 20 psi

\section{B.11 Drag Coefficient - Leading Edge Varying - Average Tare Method}




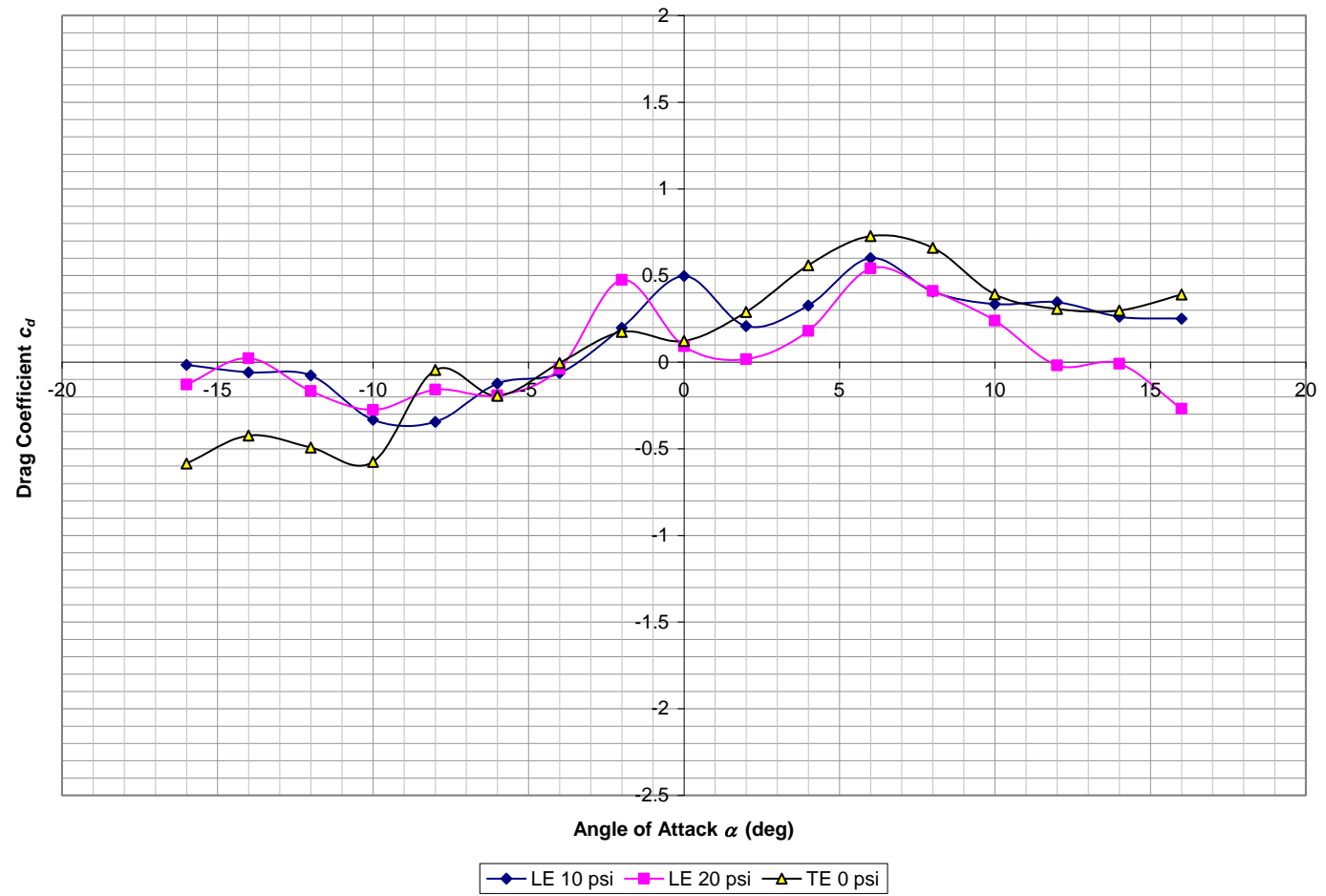

Figure B.67: Tunnel Velocity = 80 fps, Drag Coefficient vs. Angle of Attack, Leading Edge Blowing Only (average tares)

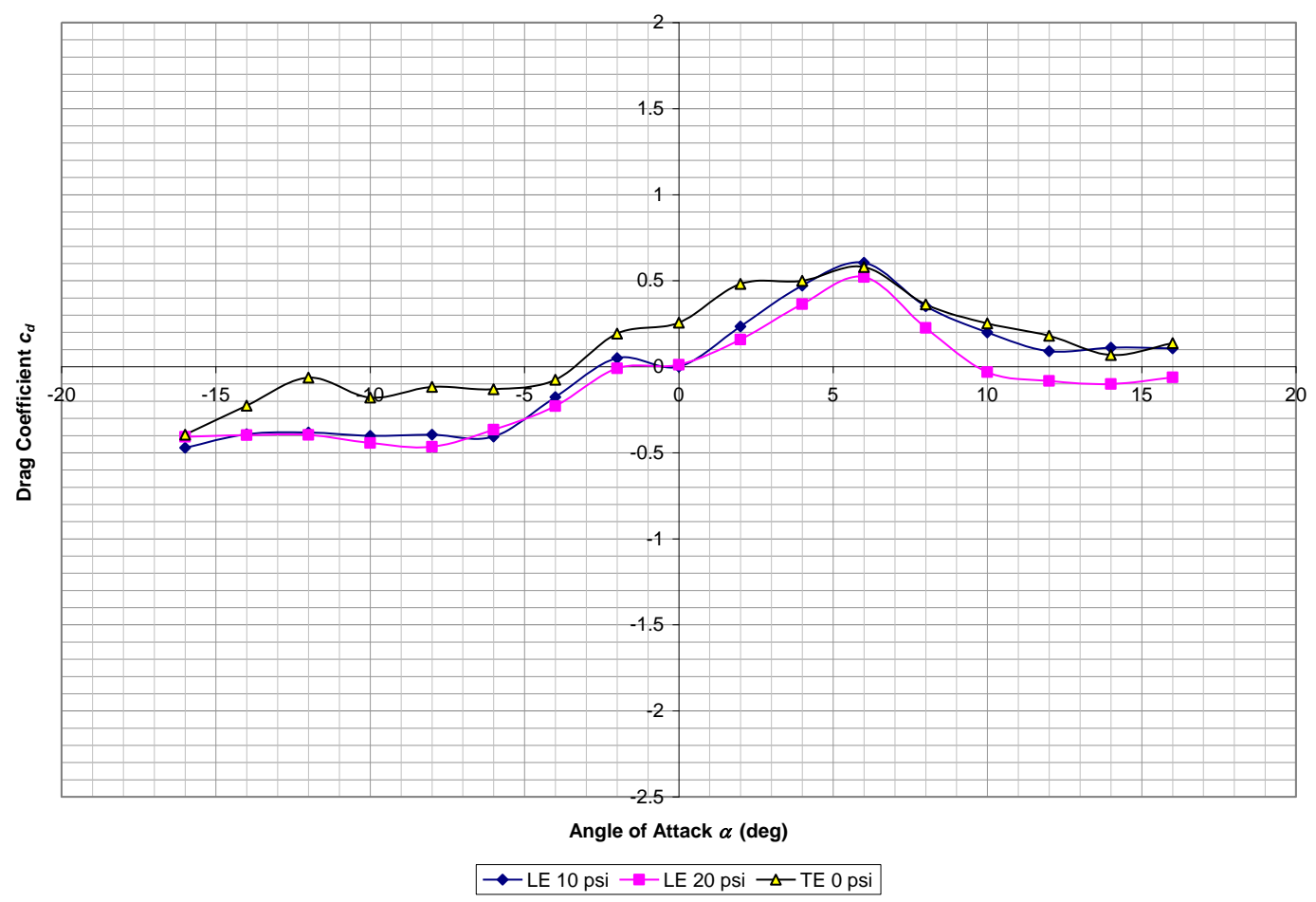

Figure B.68: Tunnel Velocity = 120 fps, Drag Coefficient vs. Angle of Attack, Leading Edge Blowing Only (average tares) 


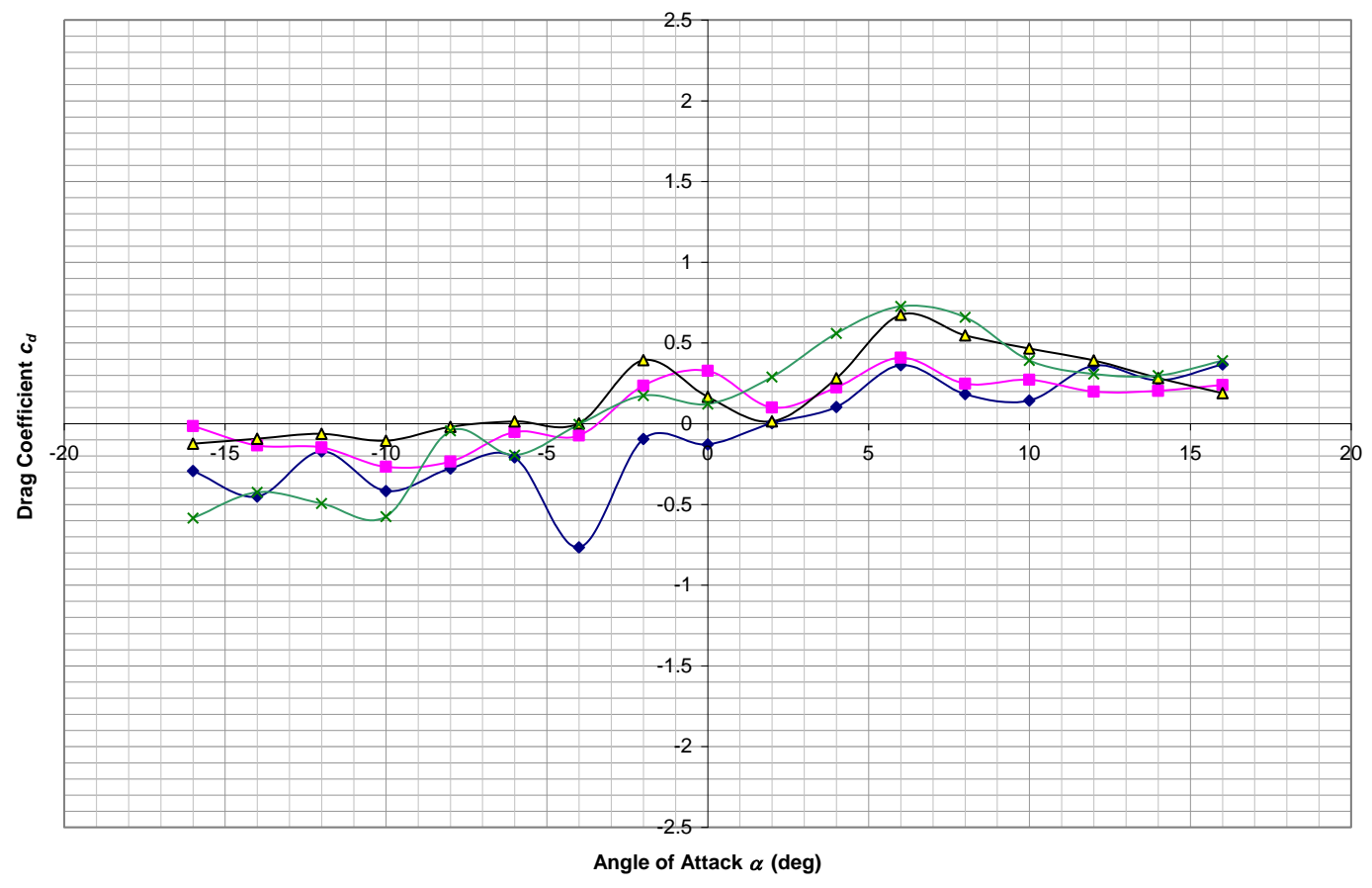

$\rightarrow-$ LE 0 psi, TE 10 psi $\rightarrow$ LE 10 psi, TE 10 psi $\multimap-$ LE 20 psi, TE 10 psi $\nrightarrow$ LE 0 psi, TE 0 psi

Figure B.69: Tunnel Velocity = 80 fps, Drag Coefficient vs. Angle of Attack, Leading Edge Varying, Trailing Edge Blowing at 10 psi (average tare)

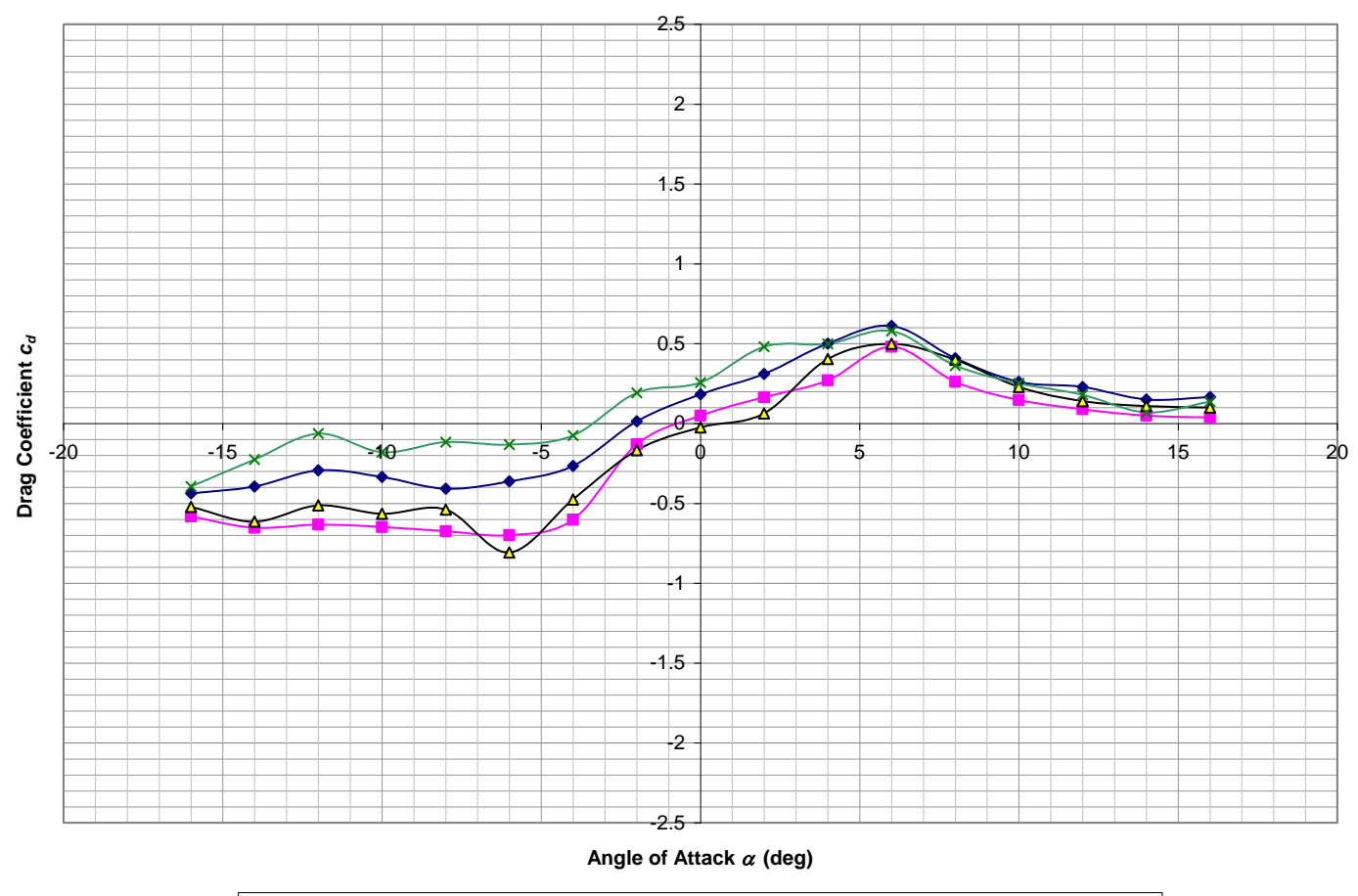

$\multimap-$ LE 0 psi, TE 10 psi $\rightarrow$ LE 10 psi, TE 10 psi $\neg-$ LE 20 psi, TE 10 psi $\rightarrow$ LE 0 psi, TE 0 psi

Figure B.70: Tunnel Velocity = 120 fps, Drag Coefficient vs. Angle of Attack, Leading Edge Varying, Trailing Edge Blowing at 10 psi (average tare) 


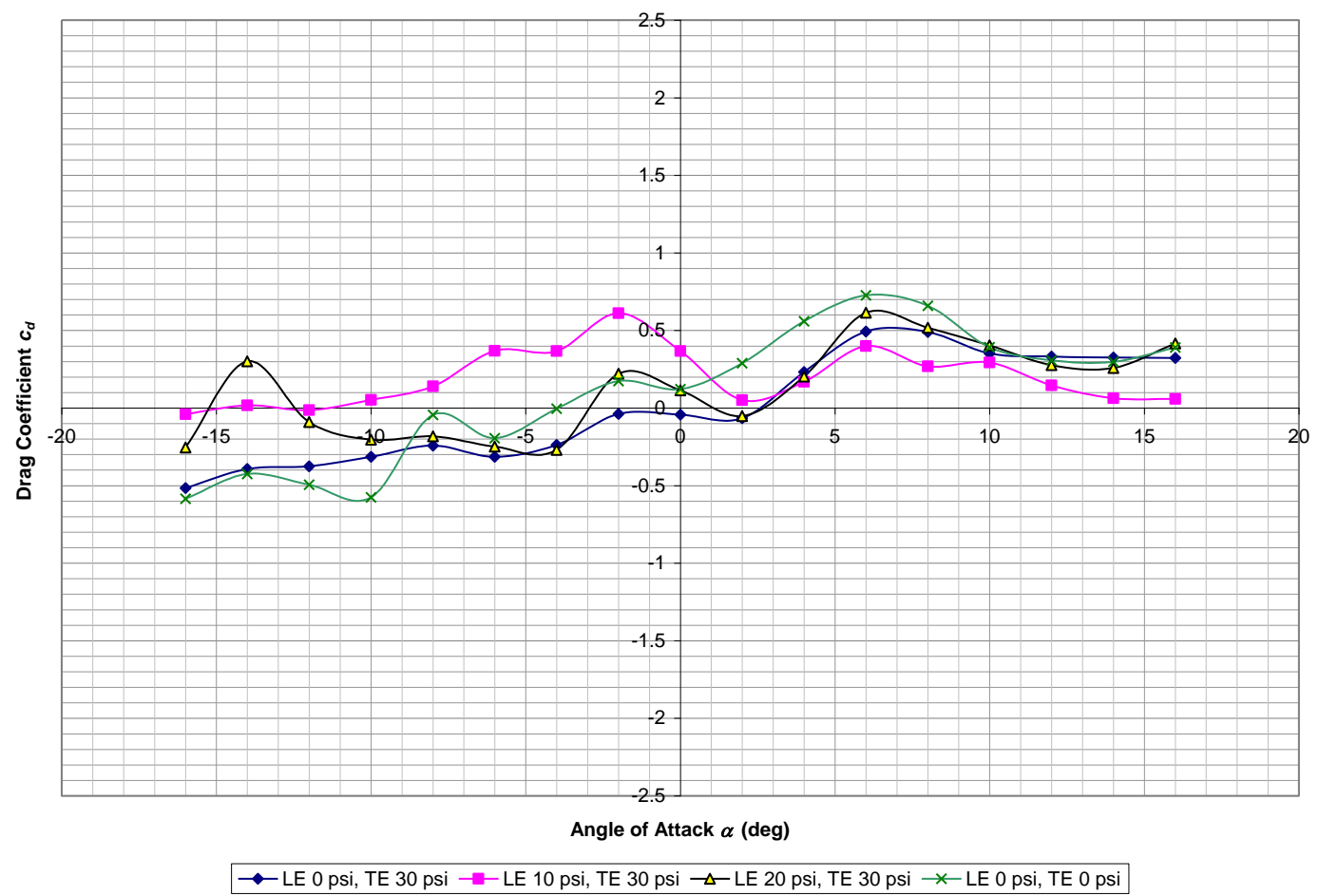

Figure B.71: Tunnel Velocity $=80$ fps, Drag Coefficient vs. Angle of Attack, Leading Edge Varying, Trailing Edge Blowing at 30 psi (average tare)

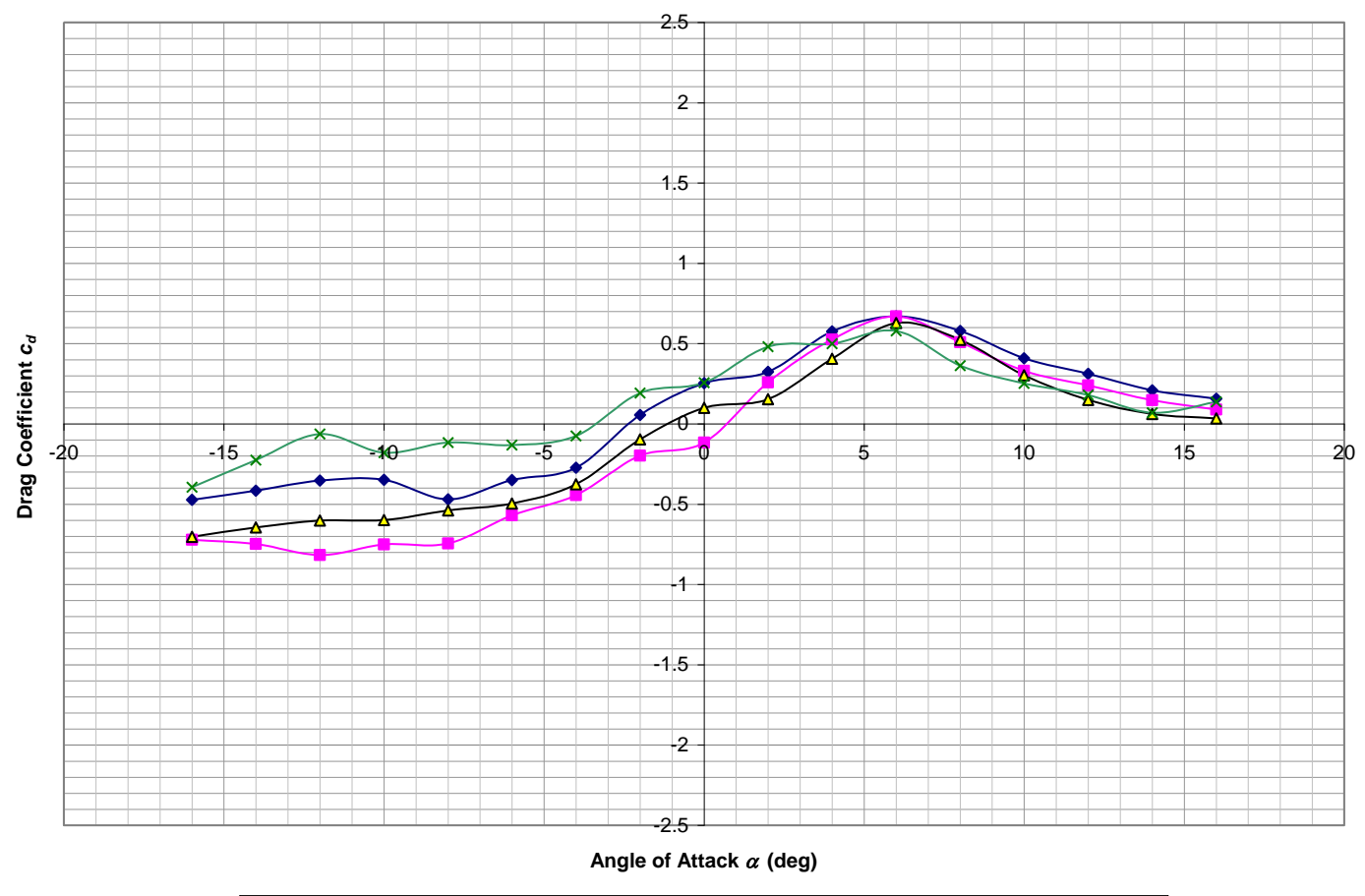

$\multimap-$ LE 0 psi, TE 30 psi $\rightarrow$ LE 10 psi, TE 30 psi $\neg-$ LE 20 psi, TE 30 psi $\rightarrow$ LE 0 psi, TE 0 psi

Figure B.72: Tunnel Velocity = 120 fps, Drag Coefficient vs. Angle of Attack, Leading Edge Varying, Trailing Edge Blowing at 30 psi (average tare)

\section{B.12 Drag Coefficient - Leading Edge Varying - Individual Tares Method}




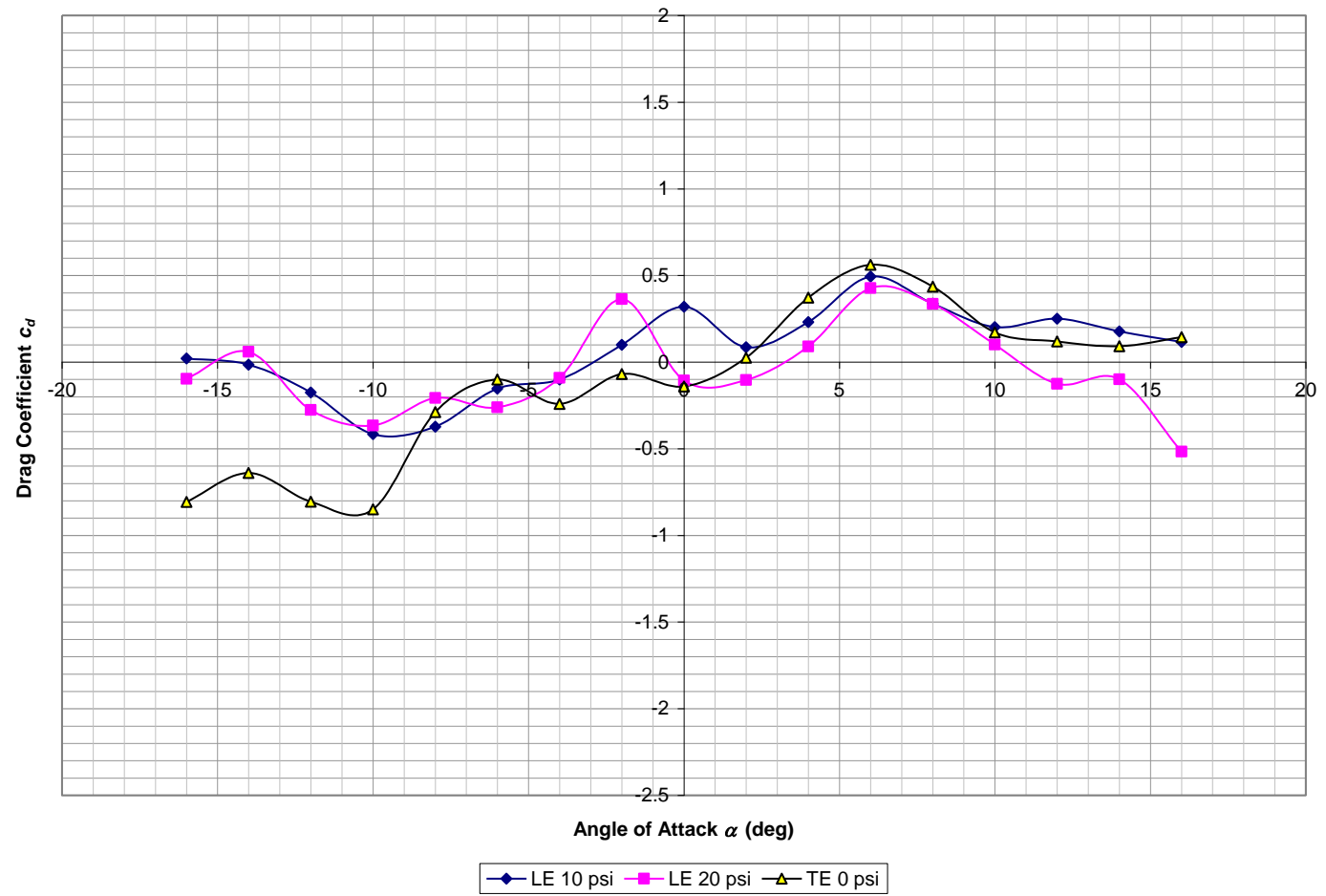

Figure B.73: Tunnel Velocity = 80 fps, Drag Coefficient vs. Angle of Attack, Leading Edge Blowing Only (individual tares)

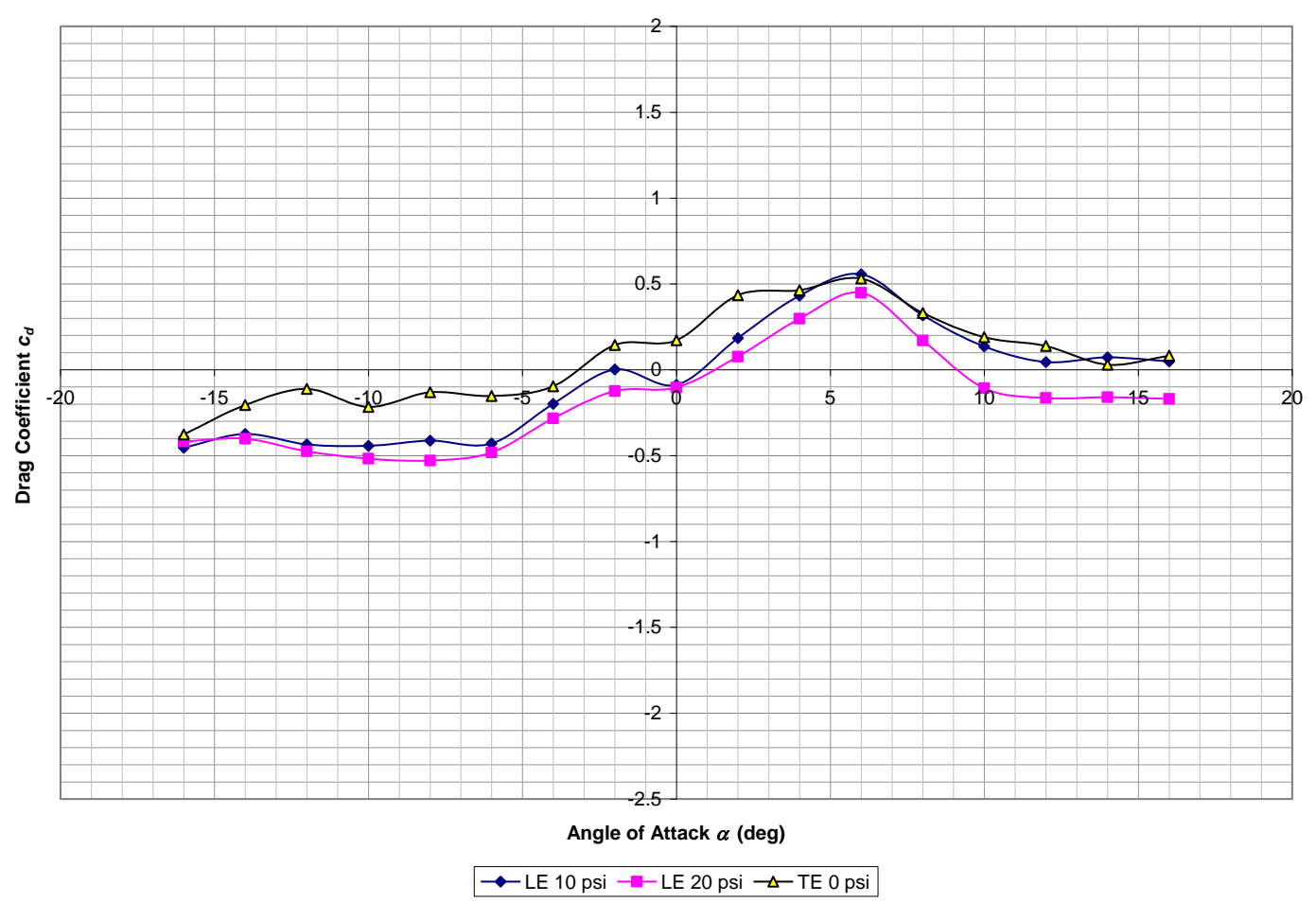

Figure B.74: Tunnel Velocity = 120 fps, Drag Coefficient vs. Angle of Attack, Leading Edge Blowing Only (individual tares) 


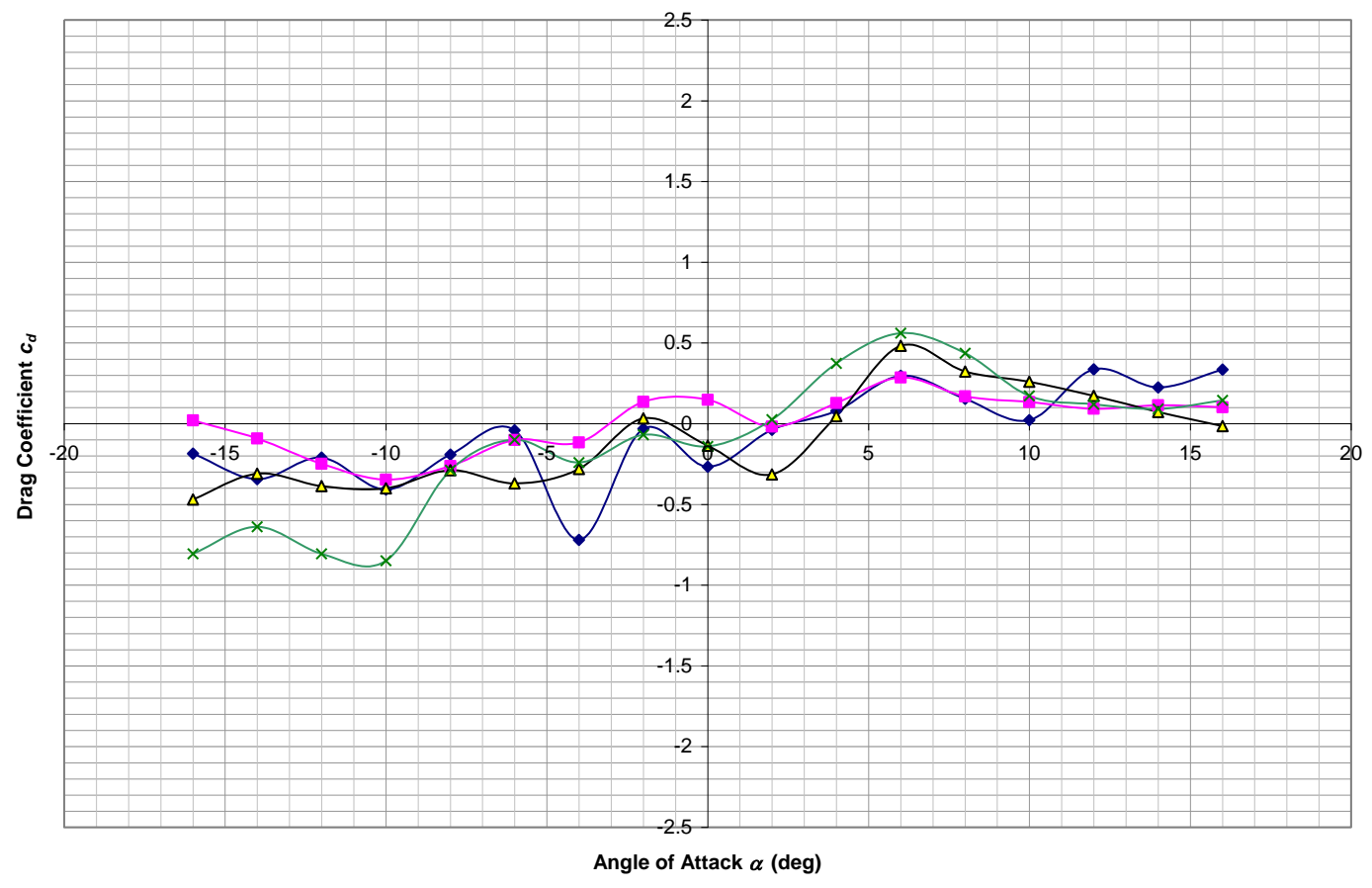

$\rightarrow-$ LE 0 psi, TE 10 psi $\rightarrow$ LE 10 psi, TE 10 psi $\multimap-$ LE 20 psi, TE 10 psi $\nrightarrow$ LE 0 psi, TE 0 psi

Figure B.75: Tunnel Velocity = 80 fps, Drag Coefficient vs. Angle of Attack, Leading Edge Varying, Trailing Edge Blowing at $10 \mathrm{psi}$ (individual tares)

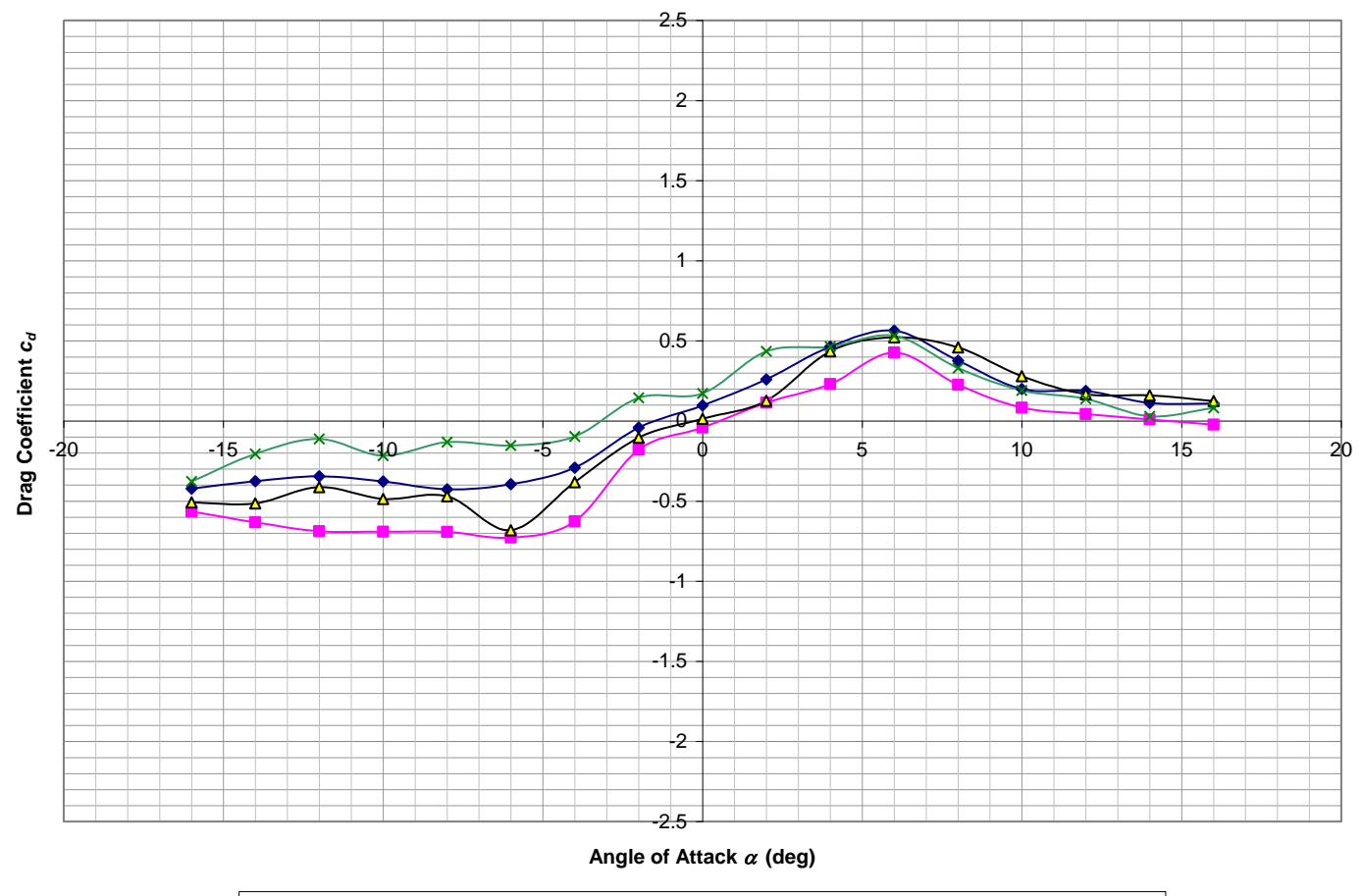

$\multimap-$ LE 0 psi, TE 10 psi $\rightarrow$ LE 10 psi, TE 10 psi $\neg-$ LE 20 psi, TE 10 psi $\rightarrow$ LE 0 psi, TE 0 psi

Figure B.76: Tunnel Velocity = 120 fps, Drag Coefficient vs. Angle of Attack, Leading Edge Varying, Trailing Edge Blowing at $10 \mathrm{psi}$ (individual tares) 


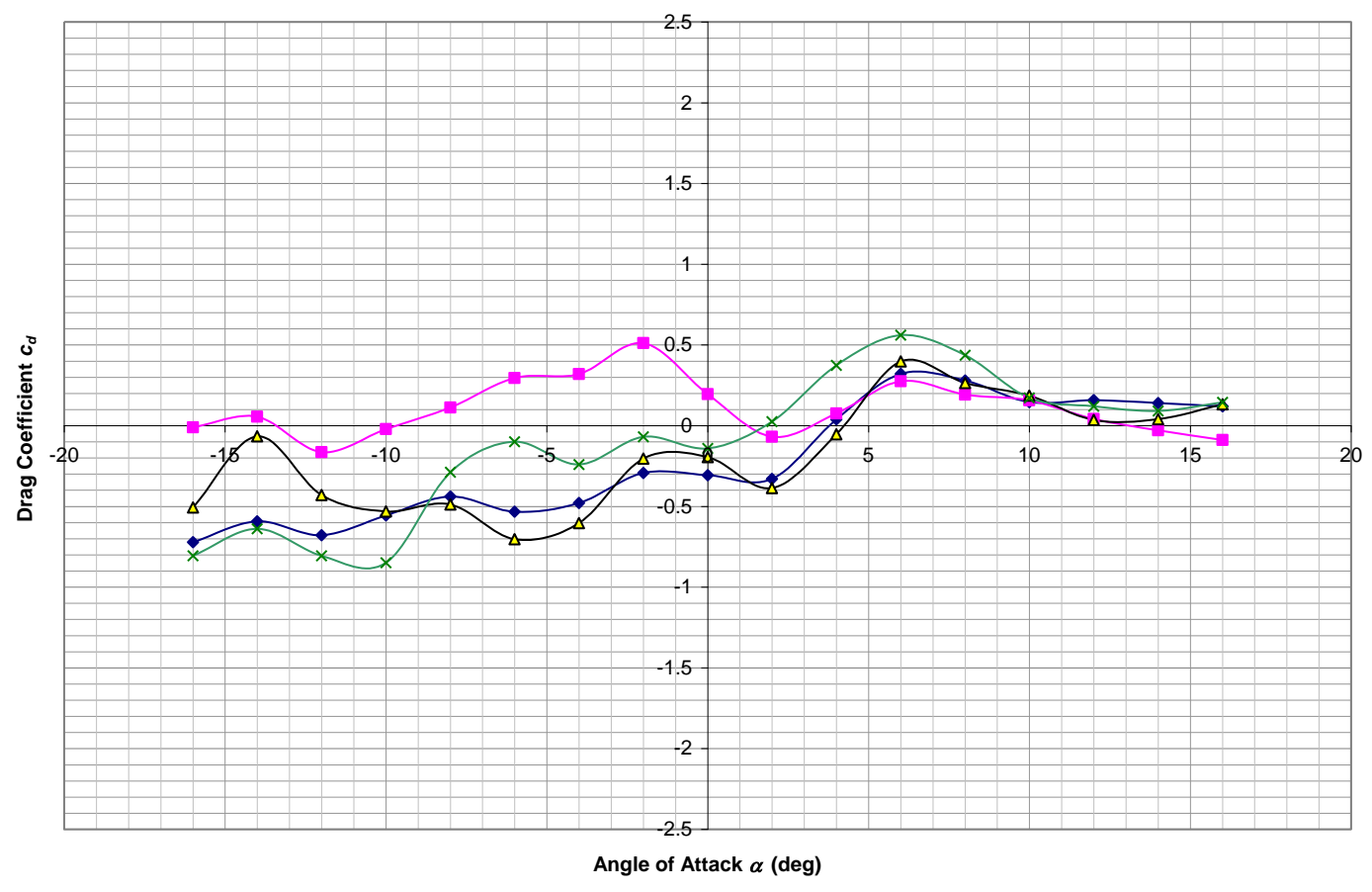

$\rightarrow-$ LE 0 psi, TE 30 psi $\rightarrow$ LE 10 psi, TE 30 psi $\neg-$ LE 20 psi, TE 30 psi $\rightarrow \leftarrow$ LE 0 psi, TE 0 psi

Figure B.77: Tunnel Velocity = 80 fps, Drag Coefficient vs. Angle of Attack, Leading Edge Varying, Trailing Edge Blowing at $30 \mathrm{psi}$ (individual tares)

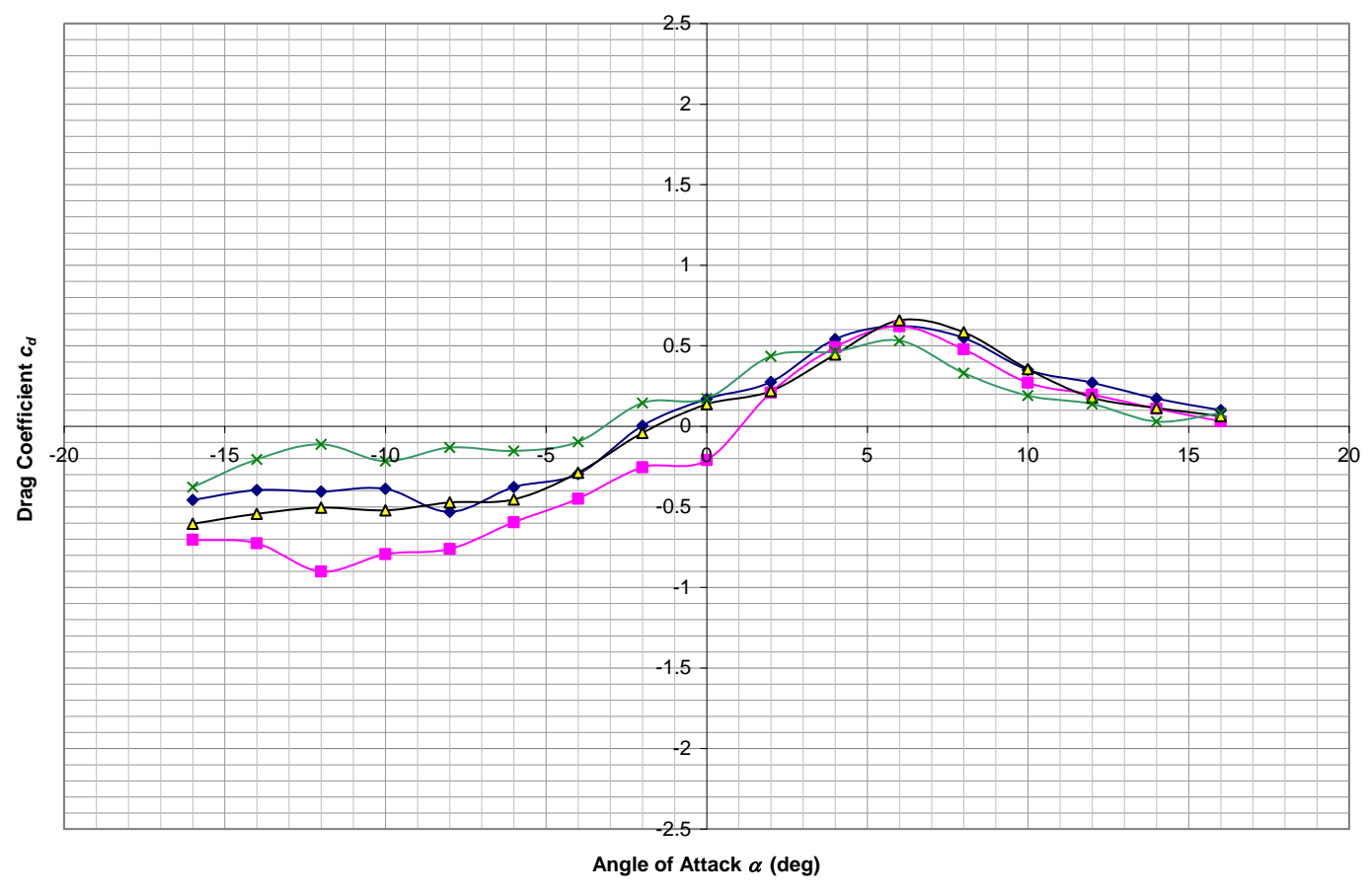

$\multimap-$ LE 0 psi, TE 30 psi $\rightarrow$ LE 10 psi, TE 30 psi $\neg-$ LE 20 psi, TE 30 psi $\rightarrow$ LE 0 psi, TE 0 psi

Figure B.78: Tunnel Velocity = 120 fps, Drag Coefficient vs. Angle of Attack, Leading Edge Varying, Trailing Edge Blowing at 30 psi (individual tares)

\section{B.13 Drag Coefficient Change - Leading Edge Varying}




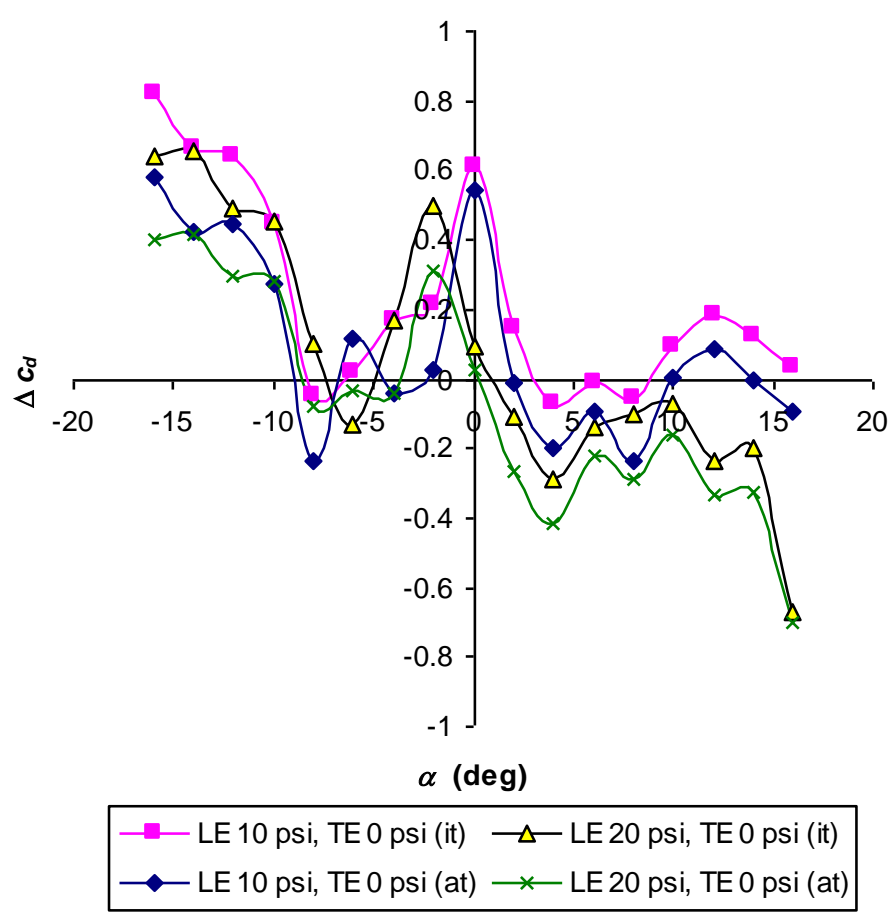

Figure B.79: Tunnel Velocity = 80 fps, Drag Coefficient Change vs. Angle of Attack, Leading Edge Blowing Only

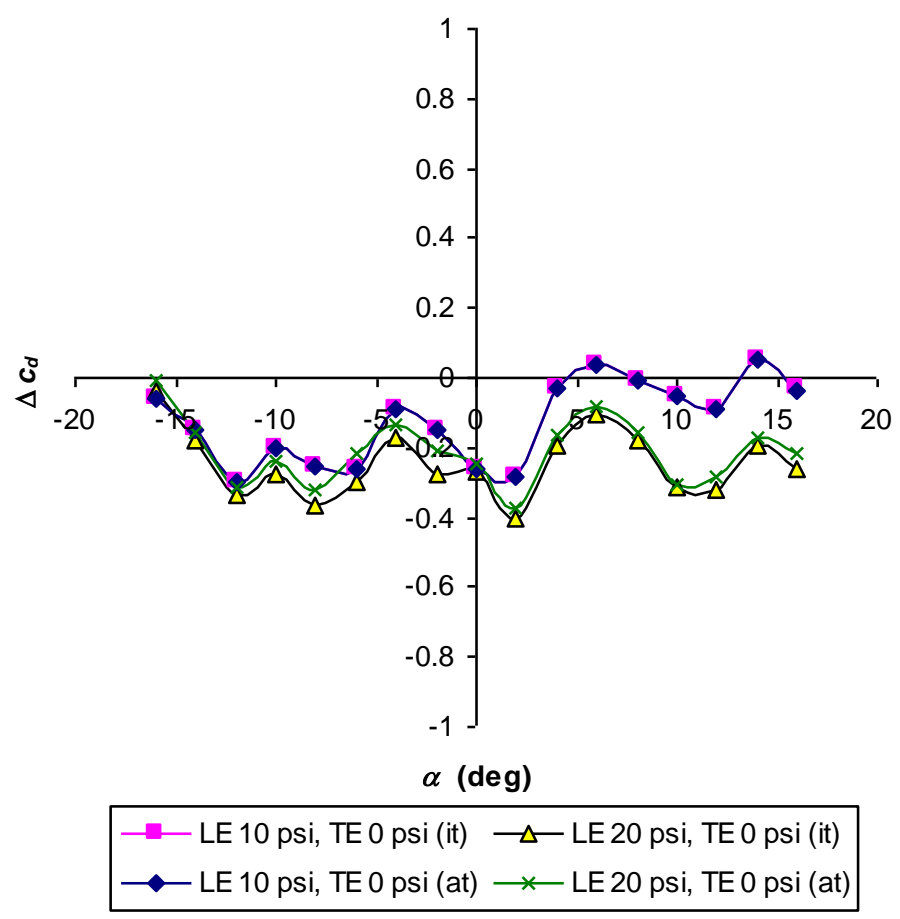

Figure B.80: Tunnel Velocity = 120 fps, Drag Coefficient Change vs. Angle of Attack, Leading Edge Blowing Only 


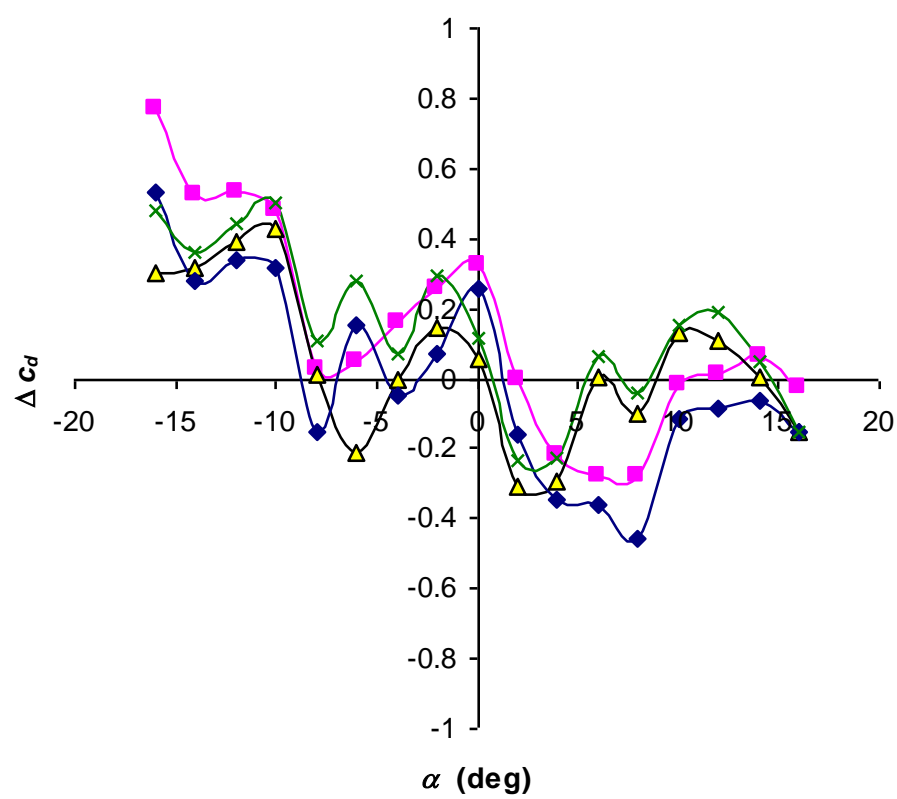

$\longrightarrow$ LE 10 psi, TE 10 psi (it) $\longleftarrow$ LE20 psi, TE 10 psi (it)
$\longrightarrow$ LE 10 psi, TE 10 psi (at) $\multimap$ LE20 psi, TE 10 psi (at)

Figure B.81: Tunnel Velocity $=80 \mathrm{fps}$, Drag Coefficient Change vs. Angle of Attack, Leading Edge Varying, Trailing Edge Blowing at 10 psi

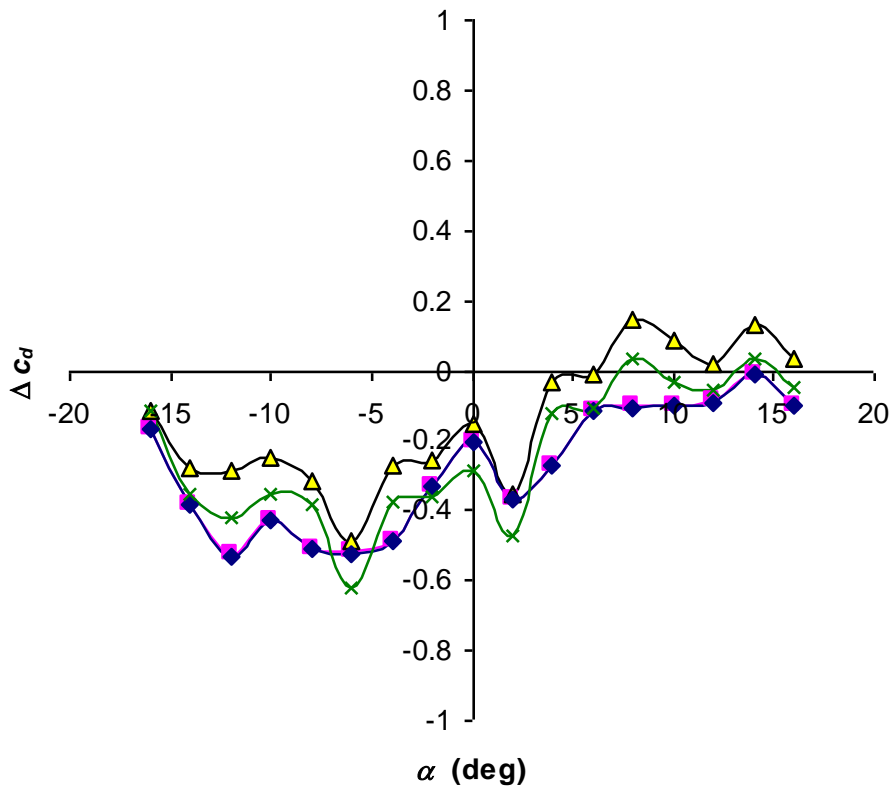

$\multimap$ LE 10 psi, TE 10 psi (it) $\longleftarrow$ LE20 psi, TE 10 psi (it)
$\multimap$ LE 10 psi, TE 10 psi (at) $\multimap$ LE 20 psi, TE 10 psi (at)

Figure B.82: Tunnel Velocity = $120 \mathrm{fps,} \mathrm{Drag} \mathrm{Coefficient} \mathrm{Change} \mathrm{vs.} \mathrm{Angle} \mathrm{of} \mathrm{Attack,} \mathrm{Leading} \mathrm{Edge} \mathrm{Varying,}$ Trailing Edge Blowing at 10 psi 


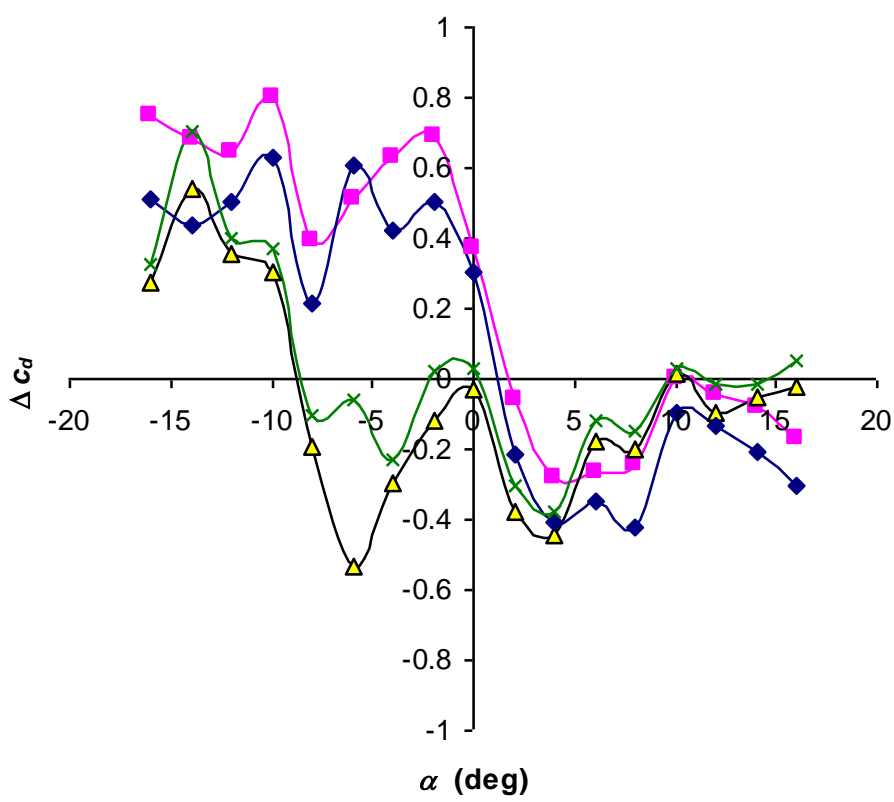

- LE 10 psi, TE 30 psi (it) $\longrightarrow$ LE20 psi, TE 30 psi (it)

$\multimap$ LE 10 psi, TE 30 psi (at) $¥$ LE20 psi, TE 30 psi (at)

Figure B.83: Tunnel Velocity = 80 fps, Drag Coefficient Change vs. Angle of Attack, Leading Edge Varying, Trailing Edge Blowing at 30 psi

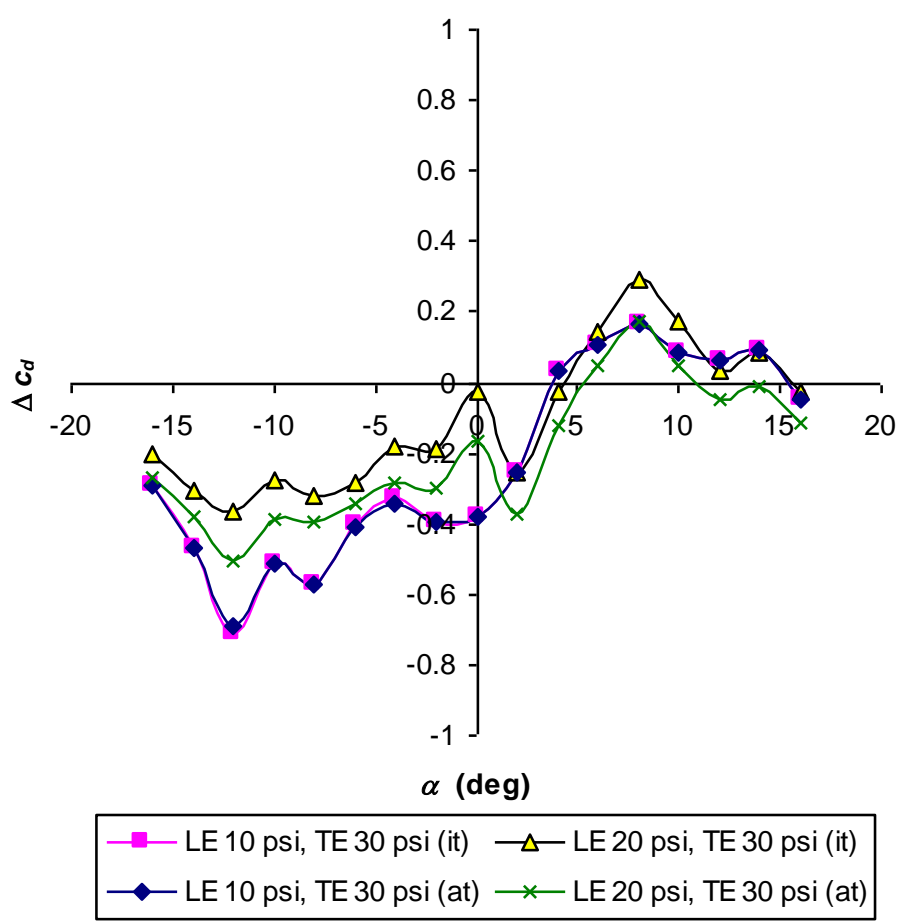

Figure B.84: Tunnel Velocity = 120 fps, Drag Coefficient Change vs. Angle of Attack, Leading Edge Varying, Trailing Edge Blowing at 30 psi 


\section{Appendix C - Short Summary for Minimum/Maximum Lift Coefficients and Stall Angles}

Table C.1 shows the a summary of results including the maximum and minimum lift coefficient, approximated stall angle-of-attack for both positive and negative stall conditions, and the stall type. The stall type is a subjective visual inspection of slope of the lift coefficient after the maximum is achieved and airfoil stalls occurs. 
Table C.1: Summary of Results

\begin{tabular}{|l|c|c|c|c|c|c|c|c|c|}
\hline \multirow{2}{*}{ Average Tare } & \multicolumn{9}{|c|}{ Tunnel Velocity $=80 \mathrm{fps}$} \\
\cline { 2 - 12 } & \multicolumn{3}{|c|}{ Leading Edge $=0 \mathrm{psi}$} & \multicolumn{3}{c|}{ Leading Edge $=10 \mathrm{psi}$} & \multicolumn{3}{c|}{ Leading Edge $=20 \mathrm{psi}$} \\
\hline Trailing Edge & $0 \mathrm{psi}$ & $10 \mathrm{psi}$ & $30 \mathrm{psi}$ & $0 \mathrm{psi}$ & $10 \mathrm{psi}$ & $30 \mathrm{psi}$ & $0 \mathrm{psi}$ & $10 \mathrm{psi}$ & $30 \mathrm{psi}$ \\
\hline$c_{l \max }$ & 1.14 & 1.68 & 1.15 & 1.16 & 1.25 & 1.34 & 1.44 & 1.35 & 1.21 \\
\hline$\alpha_{\text {stall }(+)}$ & 10 & 8 & 10 & 8 & 8 & 14 & 8 & 8 & 10 \\
\hline$c_{l \min }$ & -1.8 & -1.74 & -1.6 & -2.03 & -1.88 & -1.84 & -1.91 & -1.8 & -1.81 \\
\hline$\alpha_{\text {stall (-) }}$ & -10 & -8 & -10 & -8 & -10 & -10 & -10 & -10 & -14 \\
\hline stall type & Steep & Steep & Gradual & Steep & Gradual & $\begin{array}{c}\text { Very } \\
\text { Gradual }\end{array}$ & Gradual & Steep & Gradual \\
\hline
\end{tabular}

\begin{tabular}{|l|c|c|c|c|c|c|c|c|c|}
\hline \multirow{2}{*}{ Average Tare } & \multicolumn{10}{|c|}{ Leading Edge $=0 \mathrm{psi}$} & \multicolumn{2}{c|}{ Leading Edge $=10 \mathrm{psi}$} & \multicolumn{3}{c|}{ Leading Edge $=20 \mathrm{psi}$} \\
\cline { 2 - 12 } & $0 \mathrm{psi}$ & $10 \mathrm{psi}$ & $30 \mathrm{psi}$ & $0 \mathrm{psi}$ & $10 \mathrm{psi}$ & $30 \mathrm{psi}$ & $0 \mathrm{psi}$ & $10 \mathrm{psi}$ & $30 \mathrm{psi}$ \\
\hline Trailing Edge & 1.14 & 1.04 & 1.02 & 1.23 & 1.26 & 1.21 & 1.41 & 1.2 & 1.25 \\
\hline$c_{\text {lmax }}$ & 8 & 10 & 10 & 8 & 8 & 10 & 10 & 10 & 10 \\
\hline$\alpha_{\text {stall }(+)}$ & -1.77 & -1.67 & -1.58 & -1.68 & -1.46 & -1.35 & -1.79 & -1.6 & -1.53 \\
\hline$c_{\text {lmin }}$ & -8 & -10 & -10 & -8 & -8 & -10 & -8 & -8 & -8 \\
\hline$\alpha_{\text {stall }(-)}$ & Gradual & Gradual & $\begin{array}{c}\text { Very } \\
\text { Gradual }\end{array}$ & Steep & Steep & $\begin{array}{c}\text { Very } \\
\text { Gradual }\end{array}$ & Steep & Gradual & Gradual \\
\hline stall type & & & & & & &
\end{tabular}

\begin{tabular}{|l|c|c|c|c|c|c|c|c|c|}
\hline \multirow{2}{*}{ Individual Tare } & \multicolumn{10}{|c|}{ Tunnel Velocity $=80 \mathrm{pps}$} \\
\cline { 2 - 11 } & \multicolumn{2}{|c|}{ Leading Edge $=0 \mathrm{psi}$} & \multicolumn{2}{|c|}{ Leading Edge $=10 \mathrm{psi}$} & \multicolumn{3}{c|}{ Leading Edge $=20 \mathrm{psi}$} \\
\hline Trailing Edge & $0 \mathrm{psi}$ & $10 \mathrm{psi}$ & $30 \mathrm{psi}$ & $0 \mathrm{psi}$ & $10 \mathrm{psi}$ & $30 \mathrm{psi}$ & $0 \mathrm{psi}$ & $10 \mathrm{psi}$ & $30 \mathrm{psi}$ \\
\hline$c_{l \max }$ & 1.33 & 1.65 & 1.33 & 1.25 & 1.36 & 1.46 & 1.54 & 1.66 & 1.48 \\
\hline$\alpha_{\text {stall }(+)}$ & 10 & 8 & 10 & 8 & 8 & 14 & 8 & 8 & 10 \\
\hline$c_{l \min }$ & -1.83 & -1.86 & -1.5 & -2.01 & -1.83 & -1.82 & -1.81 & -1.56 & -1.42 \\
\hline$\alpha_{\text {stall (-) }}$ & -6 & -8 & -8 & -8 & -8 & -8 & -10 & -8 & -8 \\
\hline stall type & Steep & Steep & Steep & Steep & Gradual & $\begin{array}{c}\text { Very } \\
\text { Gradual }\end{array}$ & Gradual & Gradual & Gradual \\
\hline
\end{tabular}

\begin{tabular}{|l|c|c|c|c|c|c|c|c|c|}
\hline \multirow{2}{*}{ Individual Tare } & \multicolumn{10}{|c|}{ Leannel Velocity $=120 \mathrm{pps}$} \\
\cline { 2 - 11 } & \multicolumn{2}{|c|}{ Leang Edge $=0 \mathrm{psi}$} & \multicolumn{2}{c|}{ Leading Edge $=10 \mathrm{psi}$} & \multicolumn{2}{c|}{ Leading Edge $=20 \mathrm{psi}$} \\
\hline Trailing Edge & $0 \mathrm{psi}$ & $10 \mathrm{psi}$ & $30 \mathrm{psi}$ & $0 \mathrm{psi}$ & $10 \mathrm{psi}$ & $30 \mathrm{psi}$ & $0 \mathrm{psi}$ & $10 \mathrm{psi}$ & $30 \mathrm{psi}$ \\
\hline$c_{l \max }$ & 1.19 & 1.19 & 1.09 & 1.28 & 1.31 & 1.28 & 1.52 & 1.19 & 1.24 \\
\hline$\alpha_{\text {stall }(+)}$ & 8 & 10 & 10 & 8 & 8 & 10 & 10 & 10 & 10 \\
\hline$c_{l \min }$ & -1.76 & -1.65 & -1.54 & -1.67 & -1.45 & -1.32 & -1.7 & -1.59 & -1.51 \\
\hline$\alpha_{\text {stall (-) }}$ & -8 & -8 & -10 & -8 & -8 & -8 & -8 & -8 & -8 \\
\hline \multirow{2}{*}{ stall type } & Gradual & Gradual & $\begin{array}{c}\text { Very } \\
\text { Gradual }\end{array}$ & Steep & Steep & $\begin{array}{c}\text { Very } \\
\text { Gradual }\end{array}$ & Steep & Gradual & Gradual \\
\hline
\end{tabular}


Table C.2: Summary of Results, Alternate Representation

\begin{tabular}{|lc|c|c|c|c|c|c|c|c|}
\hline \multirow{2}{*}{ Average Tare } & \multicolumn{9}{c|}{ Tunnel Velocity $=80 \mathrm{pps}$} \\
\cline { 2 - 11 } & \multicolumn{2}{c|}{ Trailing Edge $=0 \mathrm{psi}$} & \multicolumn{2}{c|}{ Trailing Edge $=10 \mathrm{psi}$} & \multicolumn{3}{c|}{ Trailing Edge $=30 \mathrm{psi}$} \\
\hline Leading Edge & $0 \mathrm{psi}$ & $10 \mathrm{psi}$ & $20 \mathrm{psi}$ & $0 \mathrm{psi}$ & $10 \mathrm{psi}$ & $20 \mathrm{psi}$ & $0 \mathrm{psi}$ & $10 \mathrm{psi}$ & $20 \mathrm{psi}$ \\
\hline$c_{\text {lmax }}$ & 1.14 & 1.16 & 1.44 & 1.68 & 1.25 & 1.35 & 1.15 & 1.34 & 1.21 \\
\hline$\alpha_{\text {stall (+) }}$ & 10 & 8 & 8 & 8 & 8 & 8 & 10 & 14 & 10 \\
\hline$c_{\text {lmin }}$ & -1.8 & -2.03 & -1.91 & -1.74 & -1.88 & -1.8 & -1.6 & -1.84 & -1.81 \\
\hline$\alpha_{\text {stall (-) }}$ & -10 & -8 & -10 & -8 & -10 & -10 & -10 & -10 & -14 \\
\hline stall type & Steep & Steep & Gradual & Steep & Gradual & Steep & Gradual & $\begin{array}{c}\text { Very } \\
\text { Gradual }\end{array}$ & Gradual \\
\hline
\end{tabular}

\begin{tabular}{|l|c|c|c|c|c|c|c|c|c|}
\hline \multirow{2}{*}{ Average Tare } & \multicolumn{10}{|c|}{ Tunnel Velocity $=120 \mathrm{pps}$} \\
\cline { 2 - 11 } & \multicolumn{2}{|c|}{ Trailing Edge $=0 \mathrm{psi}$} & \multicolumn{2}{c|}{ Trailing Edge $=10 \mathrm{psi}$} & \multicolumn{2}{c|}{ Trailing Edge $=30 \mathrm{psi}$} \\
\hline Leading Edge & $0 \mathrm{psi}$ & $10 \mathrm{psi}$ & $20 \mathrm{psi}$ & $0 \mathrm{psi}$ & $10 \mathrm{psi}$ & $20 \mathrm{psi}$ & $0 \mathrm{psi}$ & $10 \mathrm{psi}$ & $20 \mathrm{psi}$ \\
\hline$c_{\text {lmax }}$ & 1.14 & 1.23 & 1.41 & 1.04 & 1.26 & 1.2 & 1.02 & 1.21 & 1.25 \\
\hline$\alpha_{\text {stall (+) }}$ & 8 & 8 & 10 & 10 & 8 & 10 & 10 & 10 & 10 \\
\hline$c_{\text {lmin }}$ & -1.77 & -1.68 & -1.79 & -1.67 & -1.46 & -1.6 & -1.58 & -1.35 & -1.53 \\
\hline$\alpha_{\text {stall (-) }}$ & -8 & -8 & -8 & -10 & -8 & -8 & -10 & -10 & -8 \\
\hline stall type & Gradual & Steep & Steep & Gradual & Steep & Gradual & $\begin{array}{c}\text { Very } \\
\text { Gradual }\end{array}$ & $\begin{array}{c}\text { Very } \\
\text { Gradual }\end{array}$ & Gradual \\
\hline
\end{tabular}

\begin{tabular}{|l|c|c|c|c|c|c|c|c|c|}
\hline \multirow{2}{*}{ Individual Tare } & \multicolumn{10}{|c|}{ Tunnel Velocity $=80 \mathrm{fps}$} \\
\cline { 2 - 11 } & \multicolumn{2}{|c|}{ Trailing Edge $=0 \mathrm{psi}$} & \multicolumn{2}{c|}{ Trailing Edge $=10 \mathrm{psi}$} & \multicolumn{3}{c|}{ Trailing Edge $=30 \mathrm{psi}$} \\
\hline Leading Edge & $0 \mathrm{psi}$ & $10 \mathrm{psi}$ & $20 \mathrm{psi}$ & $0 \mathrm{psi}$ & $10 \mathrm{psi}$ & $20 \mathrm{psi}$ & $0 \mathrm{psi}$ & $10 \mathrm{psi}$ & $20 \mathrm{psi}$ \\
\hline$c_{l \max }$ & 1.33 & 1.25 & 1.54 & 1.65 & 1.36 & 1.66 & 1.33 & 1.46 & 1.48 \\
\hline$\alpha_{\text {stall }(+)}$ & 10 & 8 & 8 & 8 & 8 & 8 & 10 & 14 & 10 \\
\hline$c_{l \min }$ & -1.83 & -2.01 & -1.81 & -1.86 & -1.83 & -1.56 & -1.5 & -1.82 & -1.42 \\
\hline$\alpha_{\text {stall }(-)}$ & -6 & -8 & -10 & -8 & -8 & -8 & -8 & -8 & -8 \\
\hline stall type & Steep & Steep & Gradual & Steep & Gradual & Gradual & Steep & $\begin{array}{c}\text { Very } \\
\text { Gradual }\end{array}$ & Gradual \\
\hline
\end{tabular}

\begin{tabular}{|l|c|c|c|c|c|c|c|c|c|}
\hline \multirow{2}{*}{ Individual Tare } & \multicolumn{10}{|c|}{ Tunnel Velocity $=120 \mathrm{fps}$} \\
\cline { 2 - 11 } & \multicolumn{2}{|c|}{ Trailing Edge $=0 \mathrm{psi}$} & \multicolumn{2}{c|}{ Trailing Edge $=10 \mathrm{psi}$} & \multicolumn{3}{c|}{ Trailing Edge $=30 \mathrm{psi}$} \\
\hline Leading Edge & $0 \mathrm{psi}$ & $10 \mathrm{psi}$ & $20 \mathrm{psi}$ & $0 \mathrm{psi}$ & $10 \mathrm{psi}$ & $20 \mathrm{psi}$ & $0 \mathrm{psi}$ & $10 \mathrm{psi}$ & $20 \mathrm{psi}$ \\
\hline$c_{\text {lmax }}$ & 1.19 & 1.28 & 1.52 & 1.19 & 1.31 & 1.19 & 1.09 & 1.28 & 1.24 \\
\hline$\alpha_{\text {stall (+) }}$ & 8 & 8 & 10 & 10 & 8 & 10 & 10 & 10 & 10 \\
\hline$c_{\text {lmin }}$ & -1.76 & -1.67 & -1.7 & -1.65 & -1.45 & -1.59 & -1.54 & -1.32 & -1.51 \\
\hline$\alpha_{\text {stall (-) }}$ & -8 & -8 & -8 & -8 & -8 & -8 & -10 & -8 & -8 \\
\hline stall type & Gradual & Steep & Steep & Gradual & Steep & Gradual & $\begin{array}{c}\text { Very } \\
\text { Gradual }\end{array}$ & $\begin{array}{c}\text { Very } \\
\text { Gradual }\end{array}$ & Gradual \\
\hline
\end{tabular}




\section{Appendix D - MATLAB Programs for use with WVU Closed Loop Wind Tunnel Data Acquisition Software}

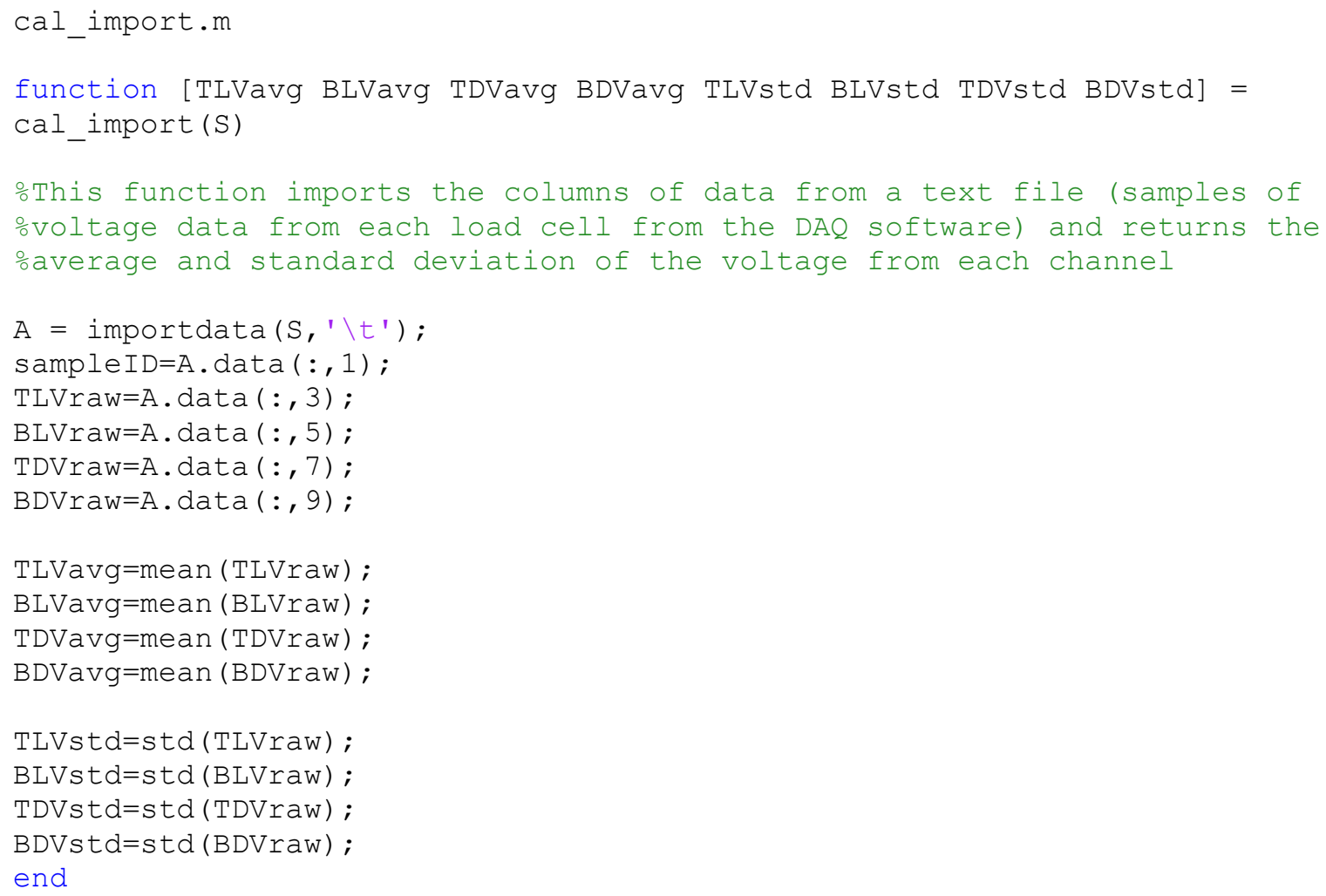




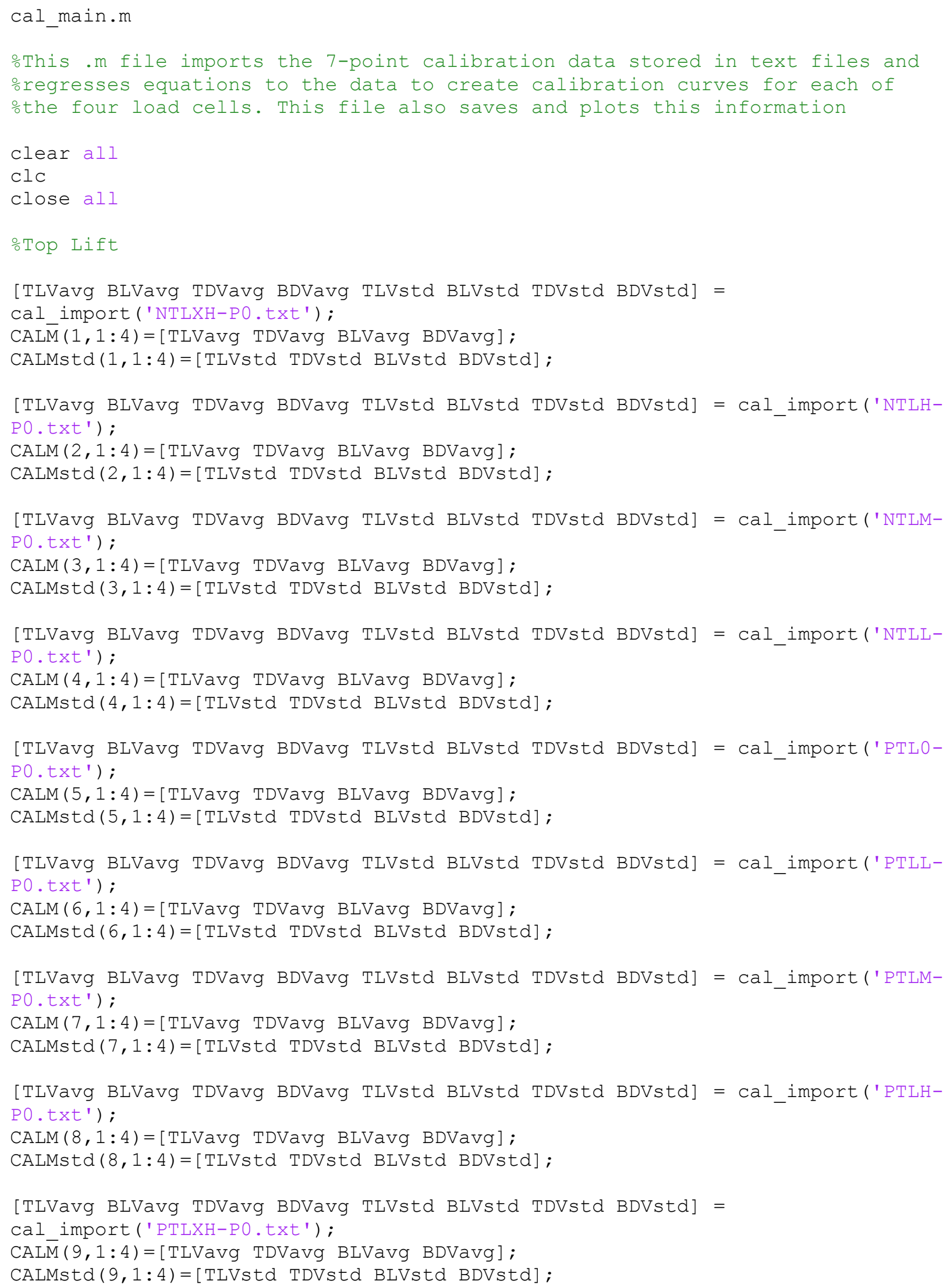




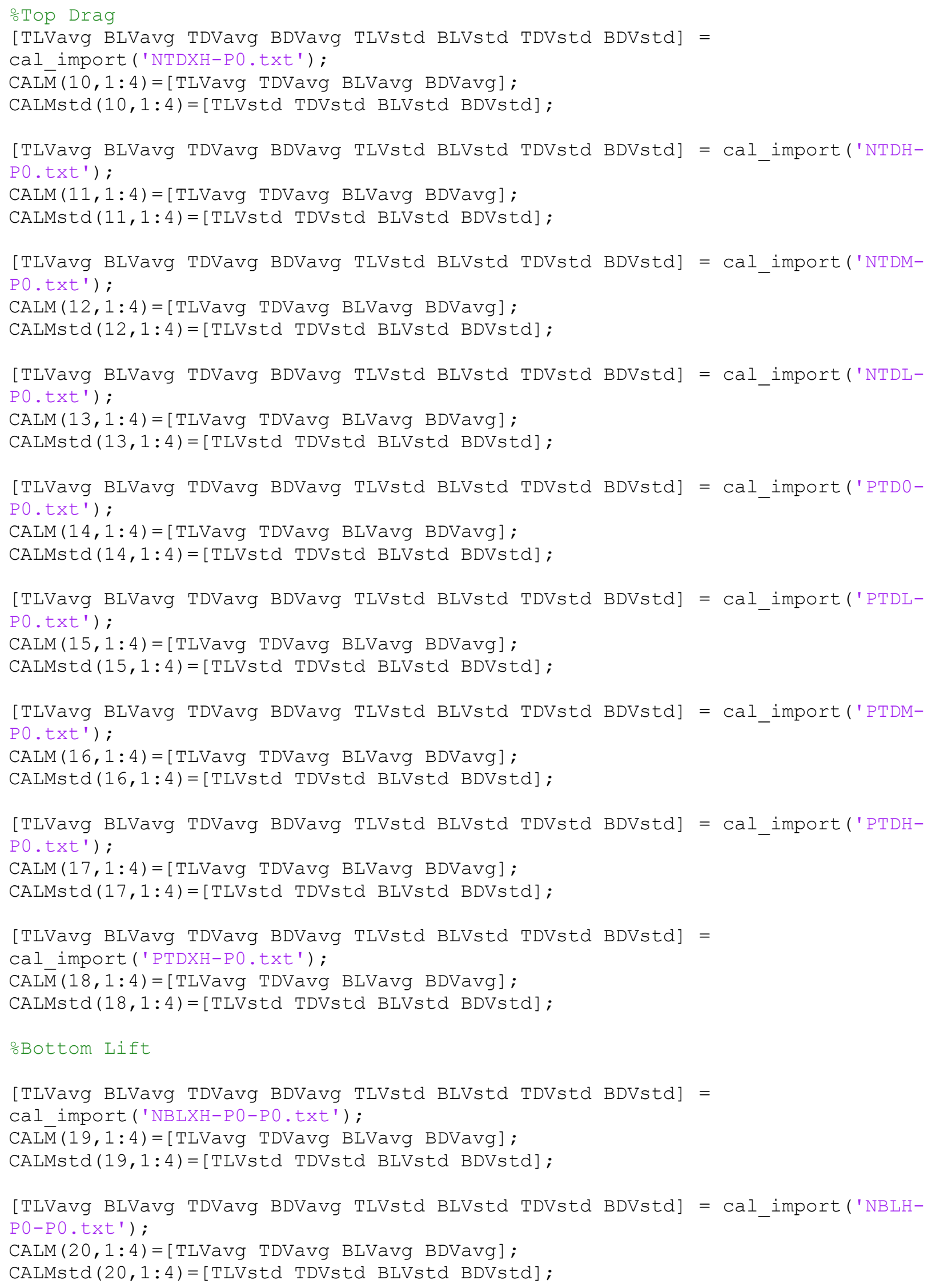


[TLVavg BLVavg TDVavg BDVavg TLVstd BLVstd TDVstd BDVstd] = cal_import('NBLM$\left.\mathrm{PO}-\mathrm{PO} . \mathrm{txt}^{\prime}\right)$;

CALM $(21,1: 4)=[$ TLVavg TDVavg BLVavg BDVavg];

CALMstd $(21,1: 4)=[$ TLVstd TDVstd BLVstd BDVstd] ;

[TLVavg BLVavg TDVavg BDVavg TLVstd BLVstd TDVstd BDVstd] = cal_import('NBLL$\left.\mathrm{PO}-\mathrm{PO} . \mathrm{txt}^{\prime}\right)$;

CALM $(22,1: 4)=[$ TLVavg TDVavg BLVavg BDVavg];

CALMstd $(22,1: 4)=[$ TLVstd TDVstd BLVstd BDVstd] ;

[TLVavg BLVavg TDVavg BDVavg TLVstd BLVstd TDVstd BDVstd] = cal_import('NBLO$\left.\mathrm{PO}-\mathrm{P} 0 . \mathrm{txt}^{\prime}\right)$;

CALM $(23,1: 4)=[$ TLVavg TDVavg BLVavg BDVavg];

CALMstd $(23,1: 4)=[$ TLVstd TDVstd BLVstd BDVstd $]$;

[TLVavg BLVavg TDVavg BDVavg TLVstd BLVstd TDVstd BDVstd] = cal_import('PBLL$\left.\mathrm{PO}-\mathrm{PO} \cdot \mathrm{txt}^{\prime}\right) ;$

CALM $(24,1: 4)=[$ TLVavg TDVavg BLVavg BDVavg];

CALMstd $(24,1: 4)=[$ TLVstd TDVstd BLVstd BDVstd] ;

[TLVavg BLVavg TDVavg BDVavg TLVstd BLVstd TDVstd BDVstd] = cal_import('PBLM$\left.\mathrm{PO}-\mathrm{PO} . \mathrm{txt}^{\prime}\right)$;

CALM $(25,1: 4)=[$ TLVavg TDVavg BLVavg BDVavg];

CALMstd $(25,1: 4)=[$ TLVstd TDVstd BLVstd BDVstd $]$;

[TLVavg BLVavg TDVavg BDVavg TLVstd BLVstd TDVstd BDVstd] = cal_import('PBLH$\left.\mathrm{PO}-\mathrm{PO} \cdot \mathrm{txt}^{\prime}\right)$;

CALM $(26,1: 4)=[$ TLVavg TDVavg BLVavg BDVavg];

CALMstd $(26,1: 4)=[$ TLVstd TDVstd BLVstd BDVstd $]$;

[TLVavg BLVavg TDVavg BDVavg TLVstd BLVstd TDVstd BDVstd] =

cal_import ('PBLXH-PO-PO.txt') ;

$\operatorname{CAL} \bar{M}(27,1: 4)=[$ TLVavg TDVavg BLVavg BDVavg];

CALMstd $(27,1: 4)=[$ TLVstd TDVstd BLVstd BDVstd $]$;

Bottom Drag

[TLVavg BLVavg TDVavg BDVavg TLVstd BLVstd TDVstd BDVstd] =

cal import ( 'NBDXH-PO.txt');

$\operatorname{CALM}(28,1: 4)=[$ TLVavg TDVavg BLVavg BDVavg] ;

CALMstd $(28,1: 4)=[$ TLVstd TDVstd BLVstd BDVstd] ;

[TLVavg BLVavg TDVavg BDVavg TLVstd BLVstd TDVstd BDVstd] = cal_import('NBDH$\left.\mathrm{PO} \cdot \mathrm{txt}^{\prime}\right) ;$

$\operatorname{CALM}(29,1: 4)=[$ TLVavg TDVavg BLVavg BDVavg];

CALMstd $(29,1: 4)=[$ TLVstd TDVstd BLVstd BDVstd] ;

[TLVavg BLVavg TDVavg BDVavg TLVstd BLVstd TDVstd BDVstd] = cal_import('NBDM$\left.\mathrm{PO} \cdot \mathrm{txt}^{\prime}\right)$;

CALM $(30,1: 4)=[$ TLVavg TDVavg BLVavg BDVavg];

CALMstd $(30,1: 4)=[$ TLVstd TDVstd BLVstd BDVstd $]$;

[TLVavg BLVavg TDVavg BDVavg TLVstd BLVstd TDVstd BDVstd] = cal_import('NBDL$\left.\mathrm{PO} \cdot \mathrm{txt}^{\prime}\right)$;

CALM $(31,1: 4)=[$ TLVavg TDVavg BLVavg BDVavg];

CALMstd $(31,1: 4)=[$ TLVstd TDVstd BLVstd BDVstd $]$; 
[TLVavg BLVavg TDVavg BDVavg TLVstd BLVstd TDVstd BDVstd] = cal_import('NBDOPO.txt');

CALM $(32,1: 4)=[$ TLVavg TDVavg BLVavg BDVavg]

CALMstd $(32,1: 4)=[$ TLVstd TDVstd BLVstd BDVstd] ;

[TLVavg BLVavg TDVavg BDVavg TLVstd BLVstd TDVstd BDVstd] = cal_import('PBDL$\left.\mathrm{PO} \cdot \mathrm{txt}^{\prime}\right)$;

CALM $(33,1: 4)=[$ TLVavg TDVavg BLVavg BDVavg];

CALMstd $(33,1: 4)=[$ TLVstd TDVstd BLVstd BDVstd] ;

[TLVavg BLVavg TDVavg BDVavg TLVstd BLVstd TDVstd BDVstd] = cal_import('PBDM$\left.\mathrm{PO} . \mathrm{txt}^{\prime}\right)$;

CALM $(34,1: 4)=[$ TLVavg TDVavg BLVavg BDVavg];

CALMstd $(34,1: 4)=[$ TLVstd TDVstd BLVstd BDVstd $]$;

[TLVavg BLVavg TDVavg BDVavg TLVstd BLVstd TDVstd BDVstd] = cal_import ('PBDHPO.txt');

CALM $(35,1: 4)=[$ TLVavg TDVavg BLVavg BDVavg];

CALMstd $(35,1: 4)=[$ TLVstd TDVstd BLVstd BDVstd] ;

[TLVavg BLVavg TDVavg BDVavg TLVstd BLVstd TDVstd BDVstd] =

cal import ('PBDXH-PO.txt') ;

CAL $\bar{M}(36,1: 4)=[$ TLVavg TDVavg BLVavg BDVavg];

CALMstd $(36,1: 4)=[$ TLVstd TDVstd BLVstd BDVstd] ;

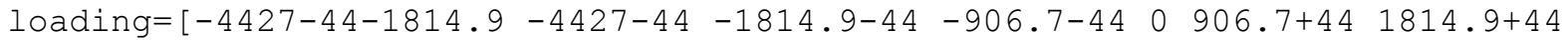

$4427+444427+44+1814.9] / 1000 * 2.2$;

CALM

save CALM_8_27_08.txt CALM -ascii -double -tabs

CALMstd

save CALM_8_27_08_std.txt CALMstd -ascii-double -tabs

TLTL is Top Lift Reading, Top Lift Applied Force

TLTD is Top Lift Reading, Top Drag Applied Force

XTLTL $=[$ ones $(\operatorname{size}(\operatorname{CALM}(1: 9,1)), 1) \operatorname{CALM}(1: 9,1)]$;

[bTLTL, bintTLTL, rTLTL, rintTLTL, statsTLTL] = regress (loading', XTLTL) ;

XTLTL=CALM $(1: 9,1)$;

StrTLTL=sprintf('y=\%g*x+\%g, $\mathrm{R}^{\wedge} 2=\% \mathrm{~g}^{\prime}, \mathrm{bTLTL}(2)$, bTLTL(1), statsTLTL (1)) ;

XTDTL $=[$ ones $(\operatorname{size}(\operatorname{CALM}(1: 9,2)), 1) \operatorname{CALM}(1: 9,2)]$;

[bTDTL, bintTDTL, rTDTL, rintTDTL, statsTDTL] = regress (loading', XTDTL);

xTDTL=CALM $(1: 9,2)$;

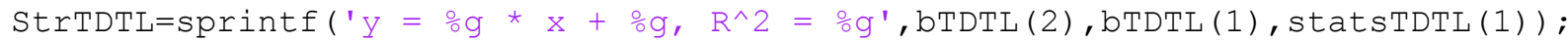

XBLTL $=[$ ones $(\operatorname{size}(\operatorname{CALM}(1: 9,3)), 1) \operatorname{CALM}(1: 9,3)]$;

[bBLTL, bintBLTL, rBLTL, rintBLTL, statsBLTL] = regress (loading', XBLTL);

XBLTL=CALM $(1: 9,3)$;

StrBLTL=sprintf(' $\left.y=\circ g * x+\frac{\circ}{\circ}, \mathrm{R}^{\wedge} 2=\circ \mathrm{g}^{\prime}, \mathrm{bBLTL}(2), \mathrm{bBLTL}(1), \operatorname{statsBLTL}(1)\right)$;

XBDTL $=[$ ones $(\operatorname{size}(\operatorname{CALM}(1: 9,4)), 1) \operatorname{CALM}(1: 9,4)]$;

[bBDTL, bintBDTL, rBDTL, rintBDTL, statsBDTL] = regress (loading',XBDTL);

xBDTL=CALM $(1: 9,4)$;

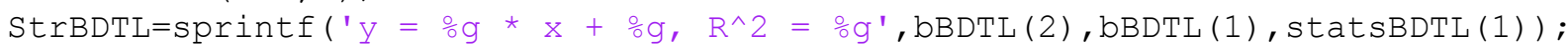


XTLTD $=[$ ones $(\operatorname{size}(\operatorname{CALM}(10: 18,1)), 1) \operatorname{CALM}(10: 18,1)]$;

[bTLTD, bintTLTD, rTLTD, rintTLTD, statsTLTD] = regress (loading', XTLTD); xTLTD=CALM $(10: 18,1)$;

StrTLTD=sprintf('y=\%g*x+\%g, $\mathrm{R}^{\wedge} 2=\% \mathrm{~g}^{\prime}, \mathrm{bTLTD}(2), \mathrm{bTLTD}(1)$, $\left.\operatorname{statsTLTD}(1)\right)$;

XTDTD $=[$ ones $(\operatorname{size}(\operatorname{CALM}(10: 18,2)), 1) \operatorname{CALM}(10: 18,2)]$;

[bTDTD, bintTDTD, rTDTD, rintTDTD, statsTDTD] = regress (loading', XTDTD) ;

$\mathrm{xTDTD}=\operatorname{CALM}(10: 18,2)$;

StrTDTD=sprintf('y=g $\left.y x+\frac{\circ}{\circ}, \mathrm{R}^{\wedge} 2=\circ \mathrm{g}^{\prime}, \mathrm{bTDTD}(2), \operatorname{bTDTD}(1), \operatorname{statsTDTD}(1)\right)$;

XBLTD $=[$ ones $(\operatorname{size}(\operatorname{CALM}(10: 18,3)), 1) \operatorname{CALM}(10: 18,3)]$;

[bBLTD, bintBLTD, rBLTD, rintBLTD, statsBLTD] = regress (loading', XBLTD);

$\mathrm{xBLTD}=\operatorname{CALM}(10: 18,3)$;

StrBLTD=sprintf('y=\%g*x+\%g, $\mathrm{R}^{\wedge} 2=\% \mathrm{~g}^{\prime}, \mathrm{bBLTD}(2), \mathrm{bBLTD}(1)$, $\left.\operatorname{statsBLTD}(1)\right)$;

$\operatorname{XBDTD}=[$ ones $(\operatorname{size}(\operatorname{CALM}(10: 18,4)), 1) \operatorname{CALM}(10: 18,4)]$;

[bBDTD, bintBDTD, rBDTD, rintBDTD, statsBDTD] = regress (loading', XBDTD) ;

$x B D T D=\operatorname{CALM}(10: 18,4)$;

StrBDTD=sprintf('y = $\left.\mathrm{g} * \mathrm{x}+\frac{\circ}{\mathrm{g}}, \mathrm{R}^{\wedge} 2=\circ \mathrm{g}^{\prime}, \mathrm{bBDTD}(2), \mathrm{bBDTD}(1), \operatorname{statsBDTD}(1)\right)$;

XTLBL $=[$ ones $(\operatorname{size}(\operatorname{CALM}(19: 27,1)), 1) \operatorname{CALM}(19: 27,1)]$;

[bTLBL, bintTLBL, rTLBL, rintTLBL, statsTLBL] = regress (loading', XTLBL);

XTLBL=CALM $(19: 27,1)$;

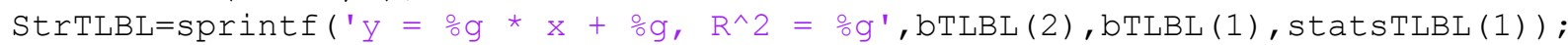

XTDBL $=[$ ones $(\operatorname{size}(\operatorname{CALM}(19: 27,2)), 1) \operatorname{CALM}(19: 27,2)]$;

[bTDBL, bintTDBL, rTDBL, rintTDBL, statsTDBL] = regress (loading', XTDBL);

$\mathrm{xTDBL}=\operatorname{CALM}(19: 27,2)$;

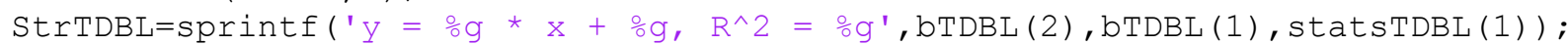

$\mathrm{XBLBL}=[$ ones $(\operatorname{size}(\operatorname{CALM}(19: 27,3)), 1) \operatorname{CALM}(19: 27,3)]$;

[bBLBL, bintBLBL, rBLBL, rintBLBL, statsBLBL] = regress (loading', XBLBL);

$x B L B L=\operatorname{CALM}(19: 27,3)$;

StrBLBL=sprintf(' $y=\circ g * x+\frac{\circ}{\circ}, R^{\wedge} 2=\circ g^{\prime}, \mathrm{bBLBL}(2), \mathrm{bBLBL}(1)$, statsBLBL (1)) ;

$\mathrm{XBDBL}=[$ ones $(\operatorname{size}(\operatorname{CALM}(19: 27,4)), 1) \operatorname{CALM}(19: 27,4)]$;

[bBDBL, bintBDBL, rBDBL, rintBDBL, statsBDBL] = regress (loading', XBDBL);

$x B D B L=C A L M(19: 27,4)$;

StrBDBL=sprintf(' $\mathrm{y}=\frac{\circ}{\mathrm{g}} * \mathrm{x}+\frac{\circ}{\mathrm{g}}, \mathrm{R}^{\wedge} 2=\circ \mathrm{g}^{\prime}, \mathrm{bBDBL}(2), \mathrm{bBDBL}(1)$, statsBDBL (1)) ;

$\mathrm{XTLBD}=[$ ones $(\operatorname{size}(\operatorname{CALM}(28: 36,1)), 1) \operatorname{CALM}(28: 36,1)]$;

[bTLBD, bintTLBD, rTLBD, rintTLBD, statsTLBD] = regress (loading', XTLBD);

$x T L B D=\operatorname{CALM}(28: 36,1) ;$

StrTLBD=sprintf(' $\left.\mathrm{y}=\frac{\circ}{\mathrm{g}} \star \mathrm{x}+\circ \mathrm{g}, \mathrm{R}^{\wedge} 2=\circ \mathrm{g}^{\prime}, \mathrm{bTLBD}(2), \mathrm{bTLBD}(1), \operatorname{statsTLBD}(1)\right)$;

$\operatorname{XTDBD}=[$ ones $(\operatorname{size}(\operatorname{CALM}(28: 36,2)), 1) \operatorname{CALM}(28: 36,2)]$;

[bTDBD, bintTDBD, rTDBD, rintTDBD, statsTDBD] = regress (loading', XTDBD);

$x T D B D=\operatorname{CALM}(28: 36,2)$;

StrTDBD=sprintf('y=\%g*x+\%g, $\mathrm{R}^{\wedge} 2=\% \mathrm{~g}^{\prime}, \mathrm{bTDBD}(2), \mathrm{bTDBD}(1)$, statsTDBD (1)) ;

$\mathrm{XBLBD}=[$ ones $(\operatorname{size}(\operatorname{CALM}(28: 36,3)), 1) \operatorname{CALM}(28: 36,3)]$;

[bBLBD, bintBLBD, rBLBD, rintBLBD, statsBLBD] = regress (loading', XBLBD) ;

$x B L B D=\operatorname{CALM}(28: 36,3)$;

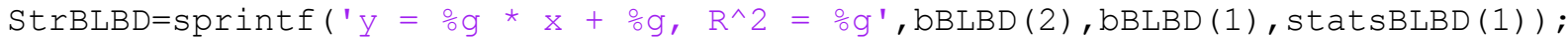

$\operatorname{XBDBD}=[$ ones $(\operatorname{size}(\operatorname{CALM}(28: 36,4)), 1) \operatorname{CALM}(28: 36,4)]$;

[bBDBD, bintBDBD, rBDBD, rintBDBD, statsBDBD] = regress (loading', XBDBD) ; 


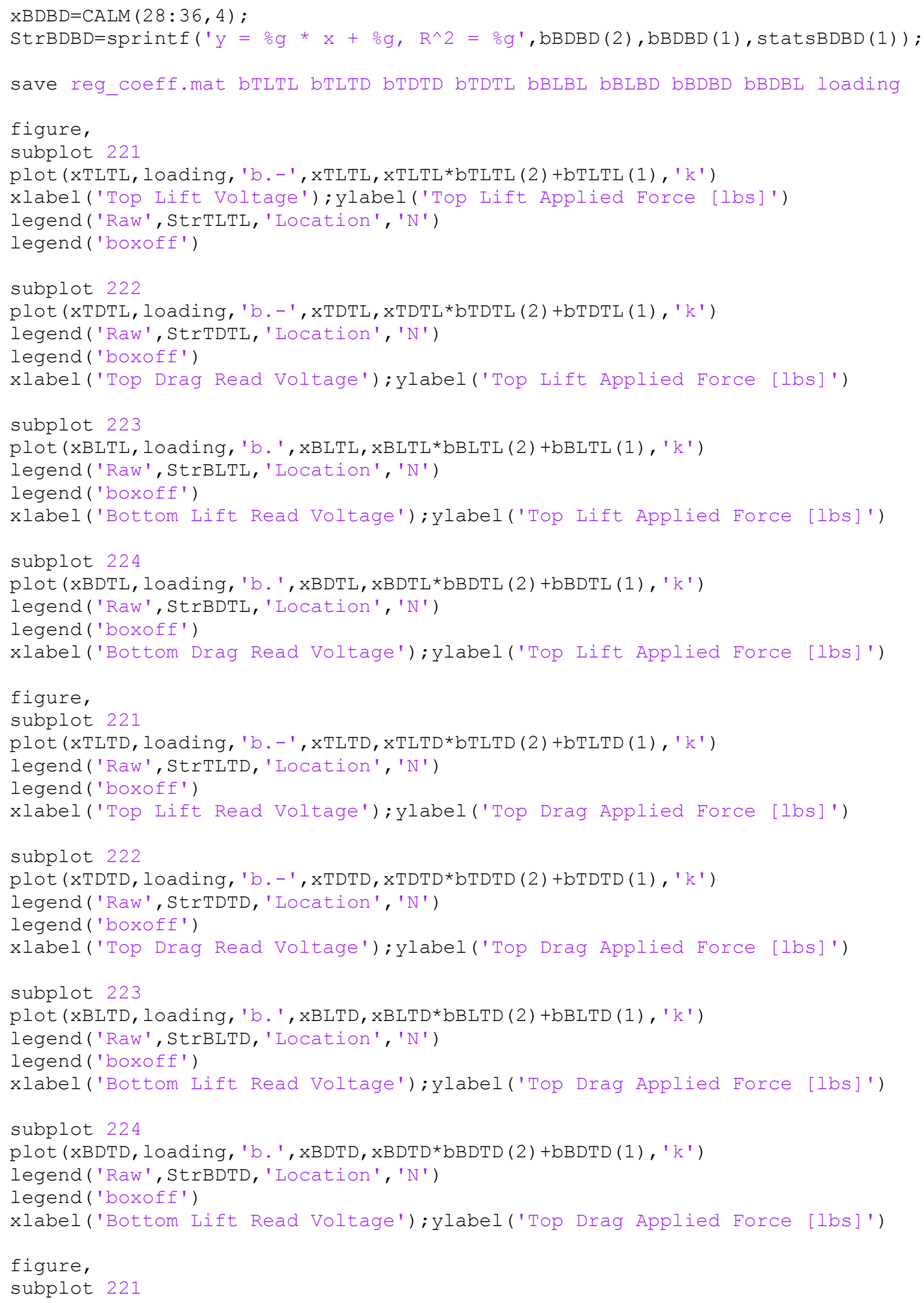




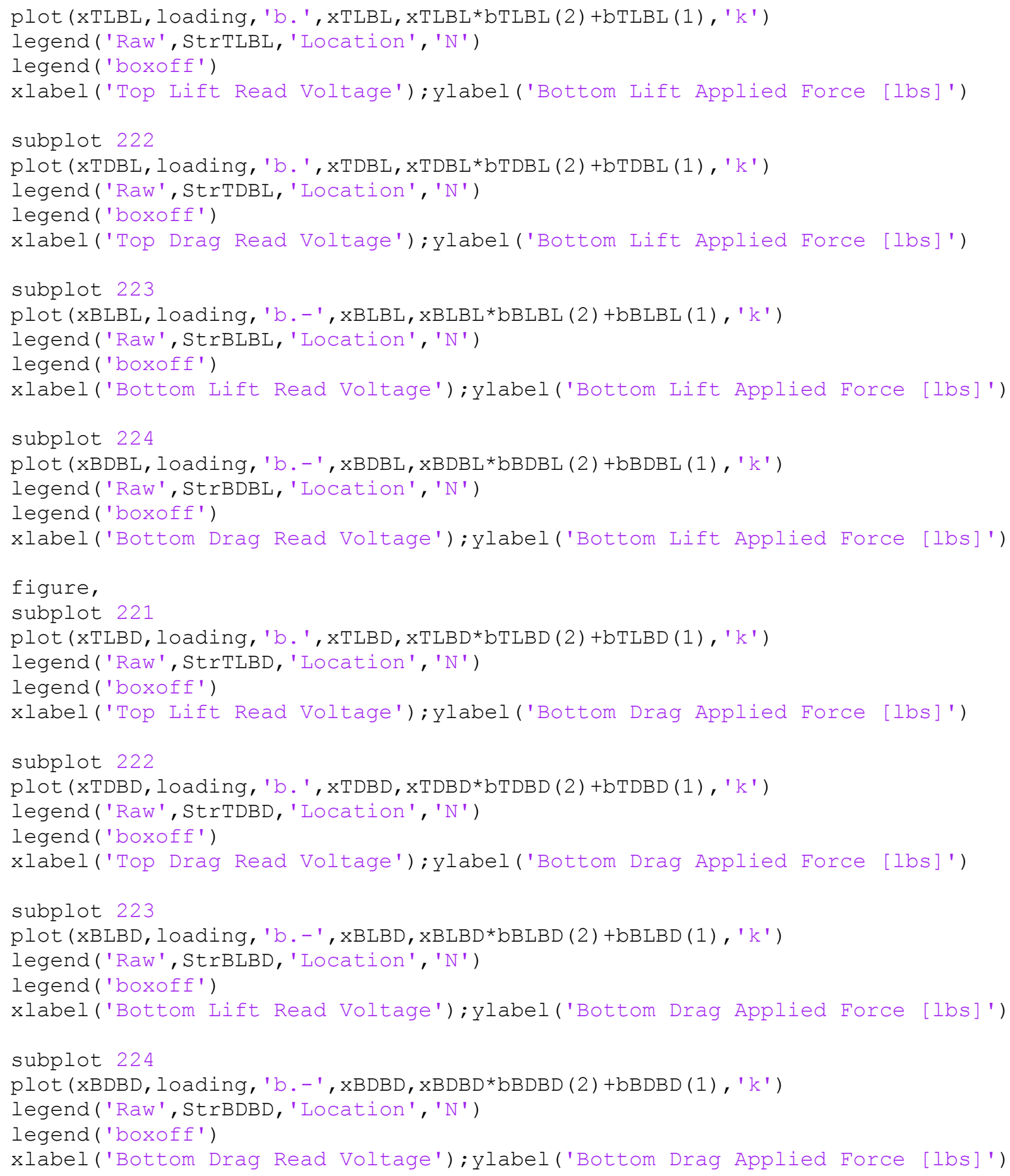




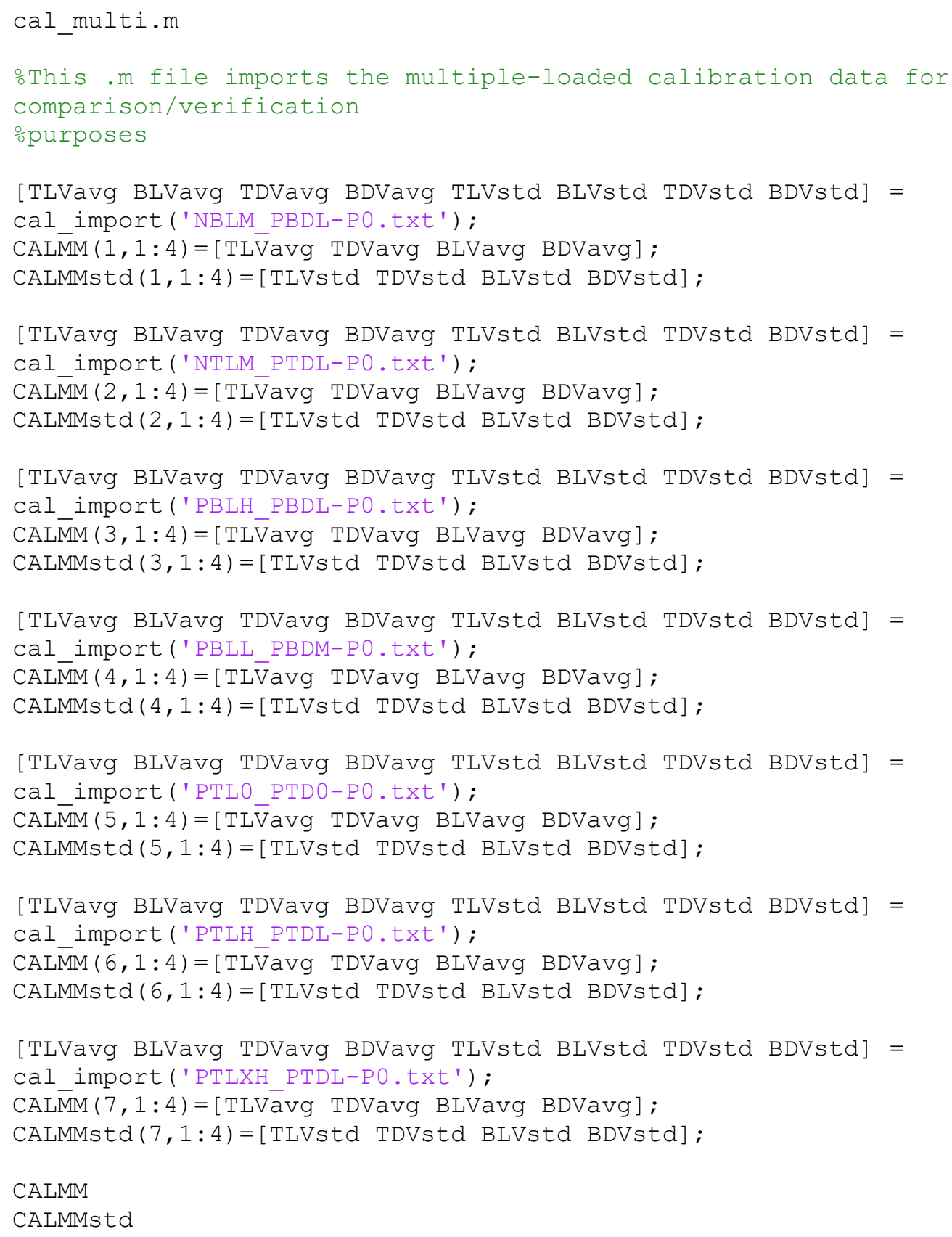




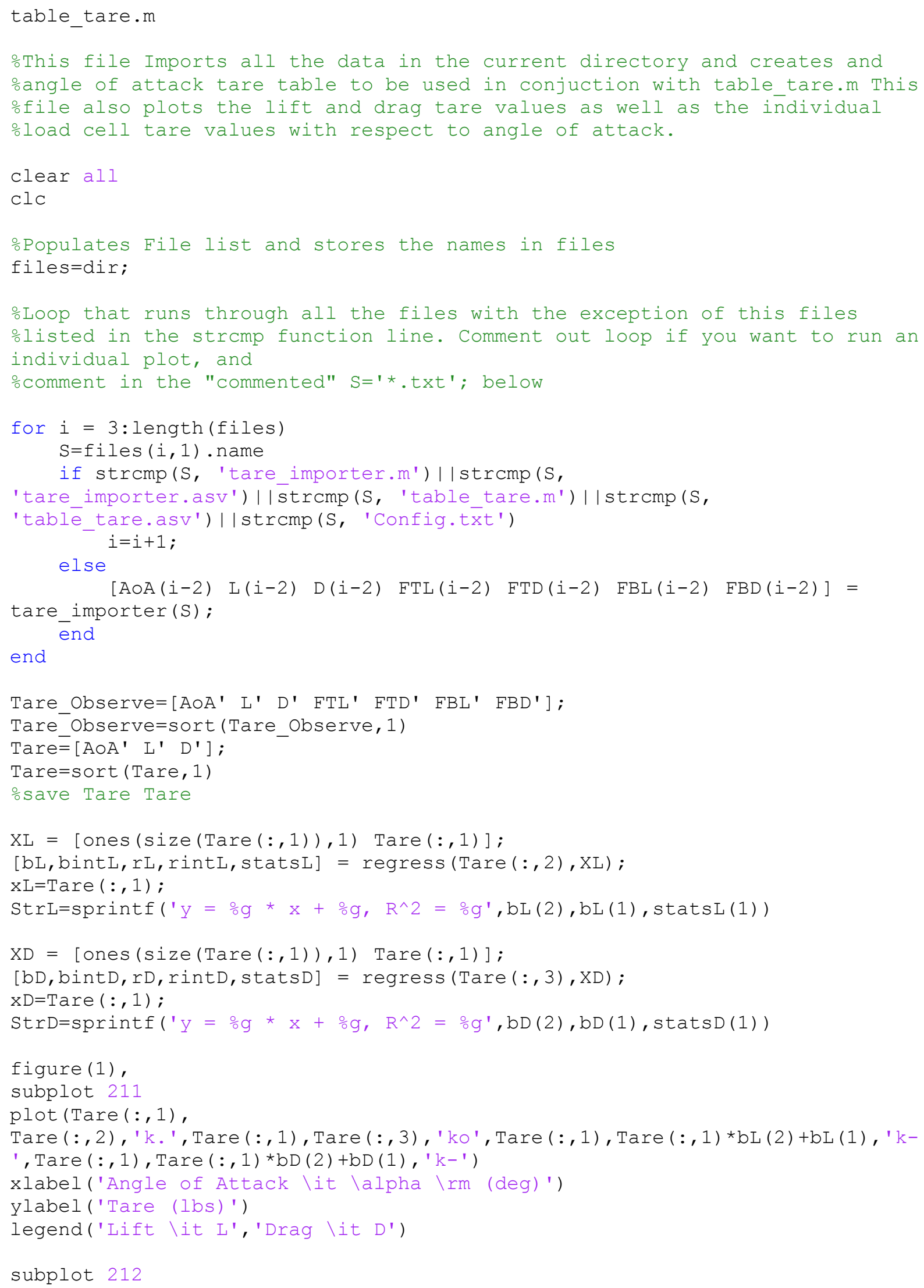


plot (Tare Observe $(:, 1)$, Tare Observe $(:, 4)$, Tare Observe $(:, 1)$, Tare Observe $(:, 5)$,

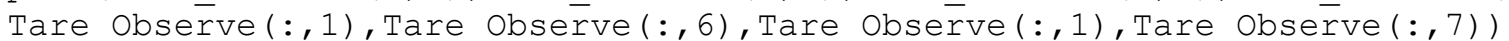

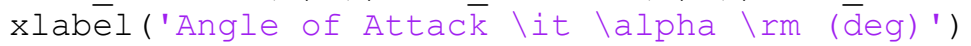
ylabel ('Tare (los)')

legend('Top Lift \it L_T','Top Drag \it D_T','Bottom Lift \it L_B', 'Bottome Drag (it D_B') 


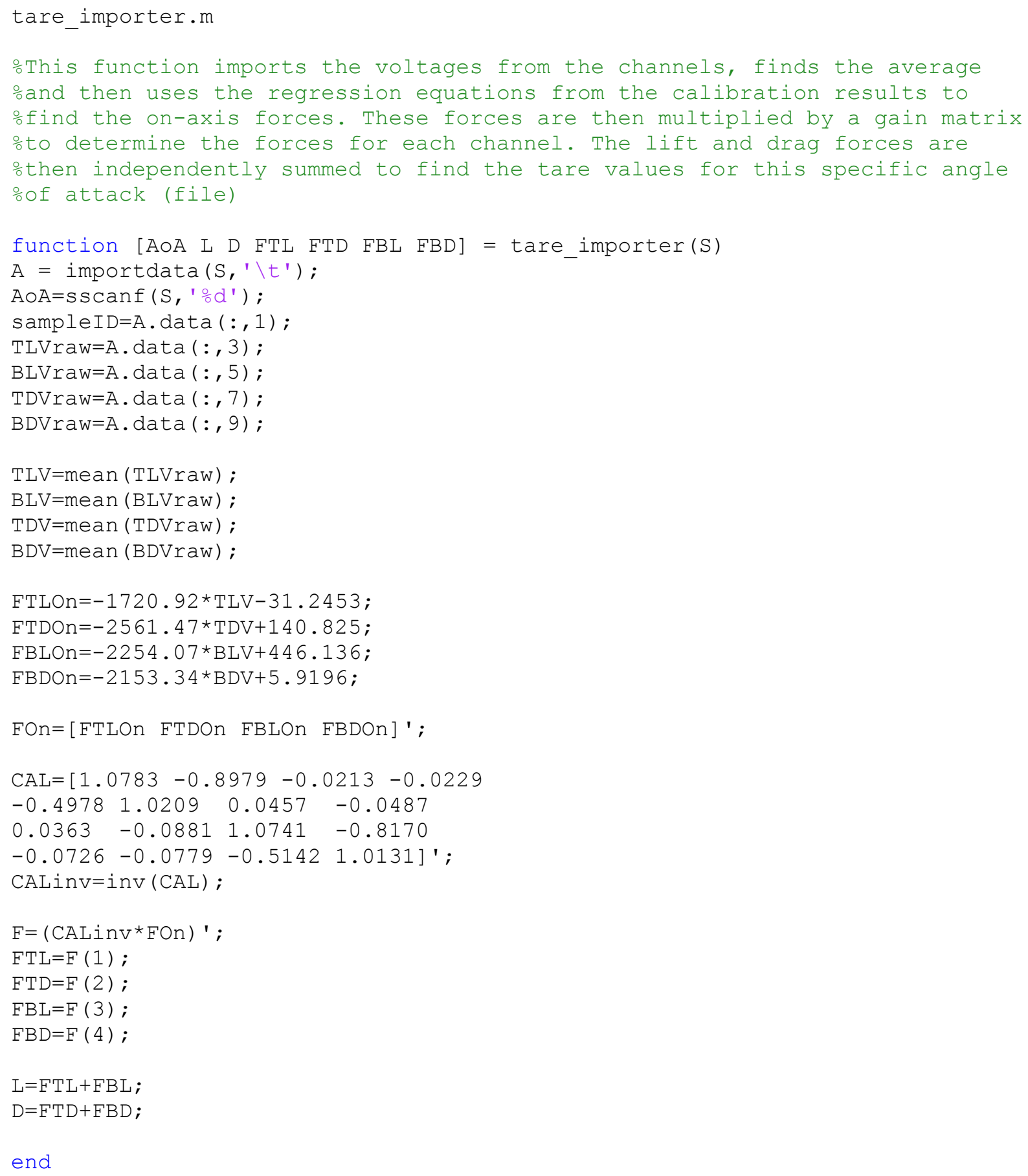

end 


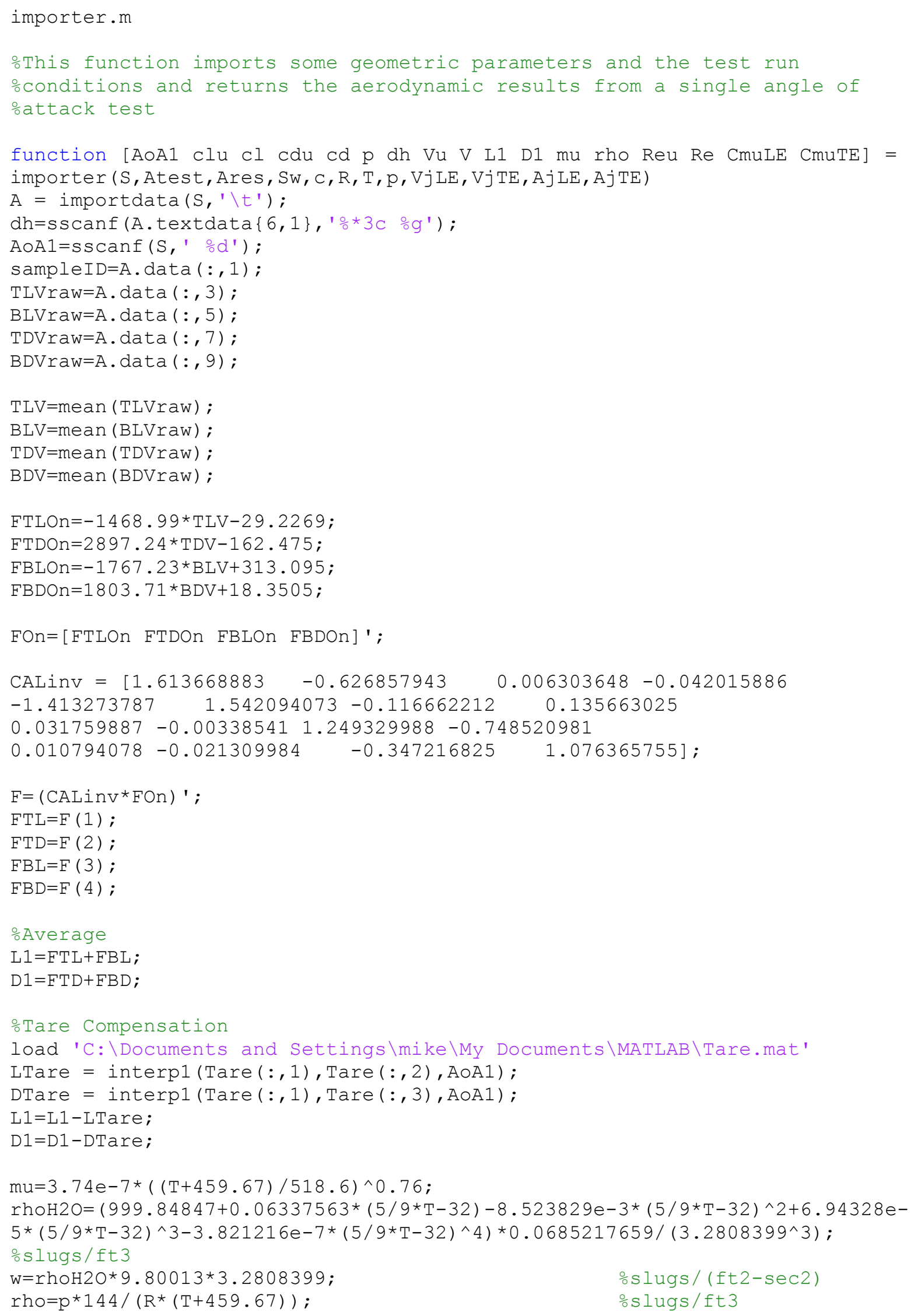




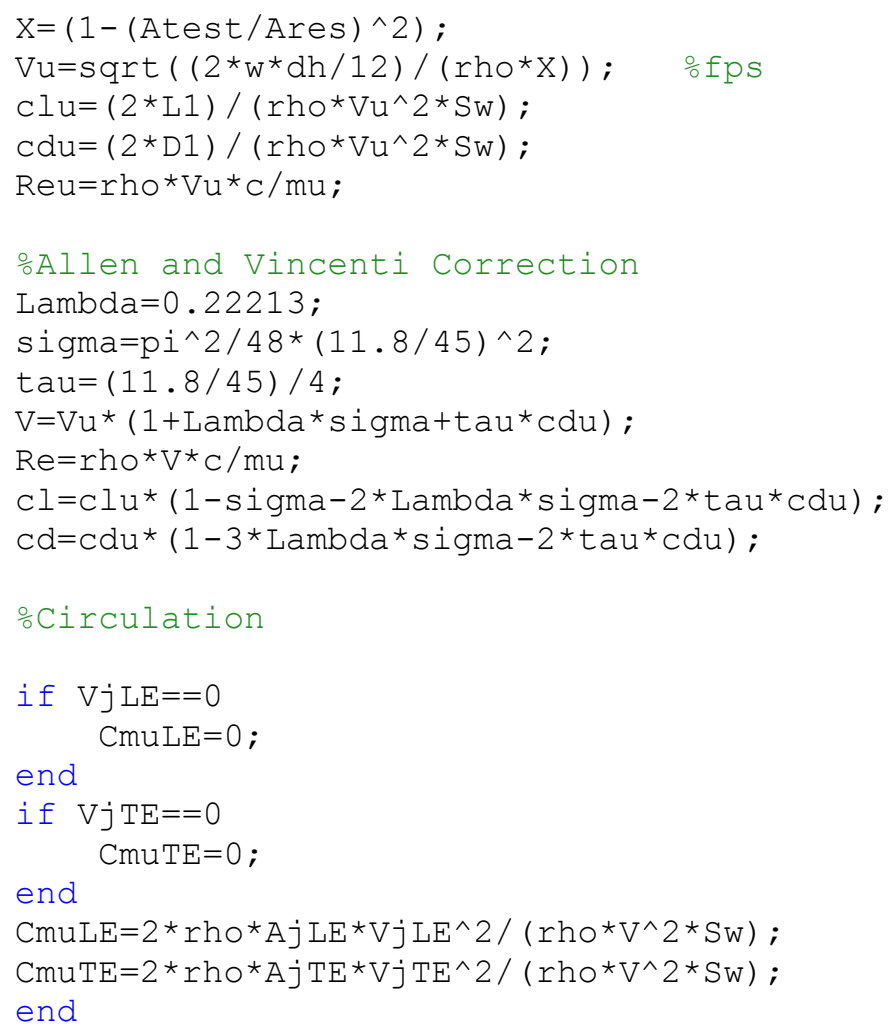




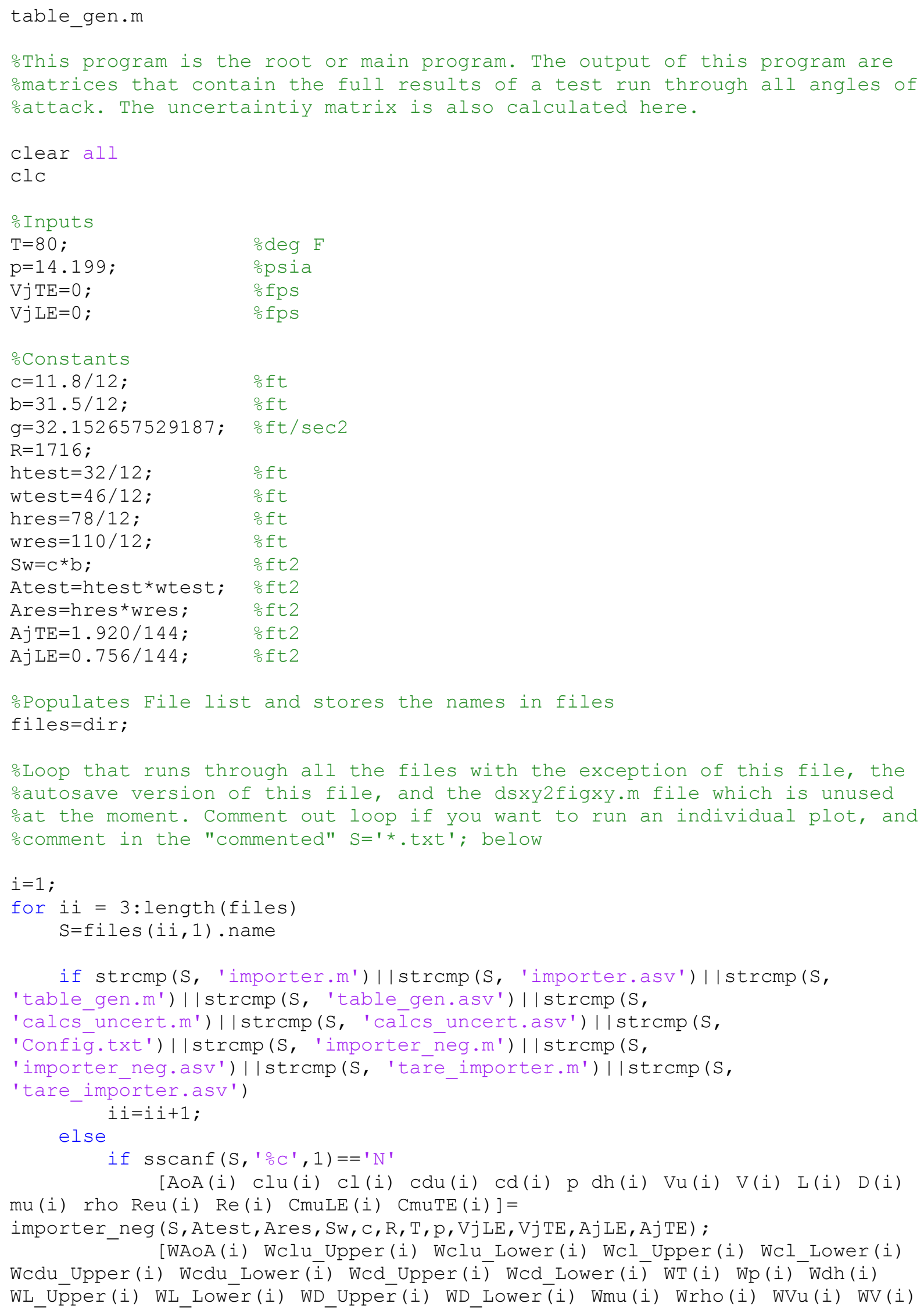


WReu(i) WRe(i) WAOA_AoA(i) Wcl_cl_Upper(i) Wcl_cl_Lower(i) Wcd_cd_Upper(i) Wcd_cd_Lower(i) WT_T(i) Wp_p (i) Wdh_dh (i) WL_L_Upper(i) WL_L_Lower (i) WD $\bar{D}$ Upper(i) WD_D_Lower(i) Wmu mu(i) Wrho_rho(i) WV_V(i)

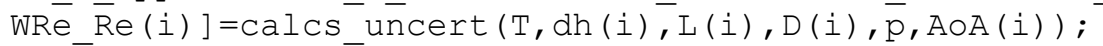

$$
\text { else }{ }^{i=i+\overline{1} \text {; }}
$$

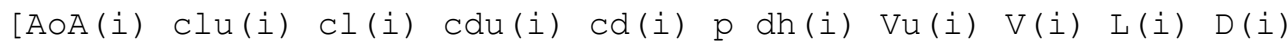

mu(i) rho Reu(i) $\operatorname{Re}(i)$ CmuLE(i) CmuTE(i)] =

importer (S, Atest, Ares, Sw, c, R, T, p, VjLE,VjTE,AjLE, AjTE) ;

[WAoA (i) Wclu_Upper(i) Wolu_Lower(i) Wol_Upper(i) Wcl_Lower(i)

Wcdu_Upper(i) Wcdu_Lower(i) Wcd_Upper(i) Wcd_Lower (i) WT (i) Wp (i) Wdh (i)

WL_Upper (i) WL_Lower(i) WD_Upper (i) WD_Lower(i) Wmu(i) Wrho(i) WVu(i) WV(i)

WRēu(i) WRe(i) WAOA_AoA (i) Wcl_cl_Upper (i) Wcl_cl_Lower(i) Wcd_cd_Upper(i)

Wcd_cd_Lower(i) WT_T (i) Wp_p (i) Wōh_dh (i) WL_L_Upper (i) WL_L_Lower $\bar{r}(i)$

WD_D_Upper(i) WD_D_Lower(i) Wmu_mu(i) Wrho_rho(i) WV_V(i)

WRe $\left.\bar{e} \_\overline{R e}(i)\right]=$ calcs_uncert $(T, \operatorname{dh}(i), \bar{L}(i), D(i), \bar{p}, \mathrm{~A} \circ \mathrm{A}(i))$;

$$
i=i+\overline{1}
$$

end

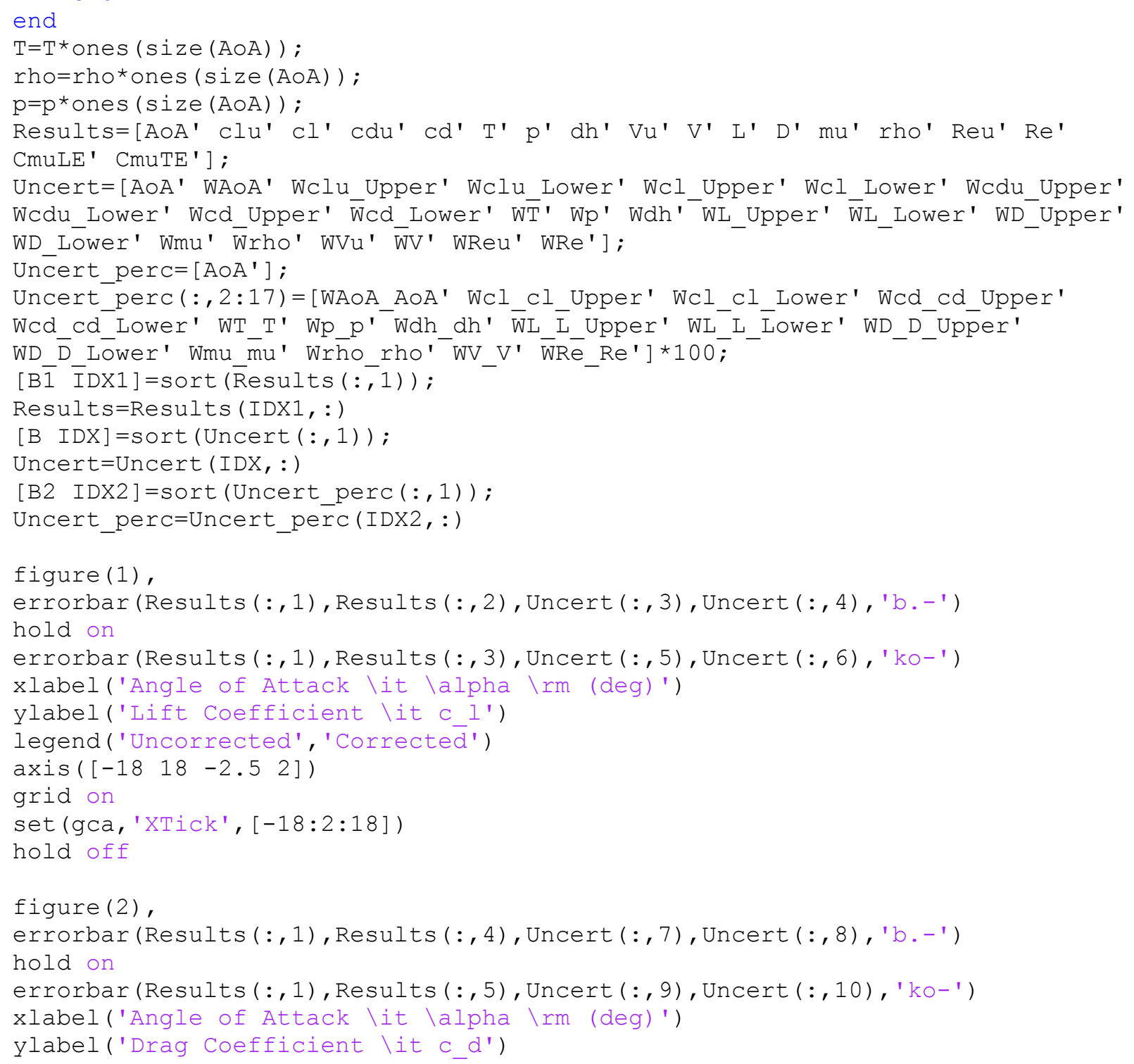


legend ('Uncorrected ', ' Corrected' )

$\operatorname{axis}\left(\left[\begin{array}{llll}-18 & 18 & -2.5 & 2\end{array}\right]\right)$

grid on

set (gca, 'XTick', [-18:2:18])

hold off 


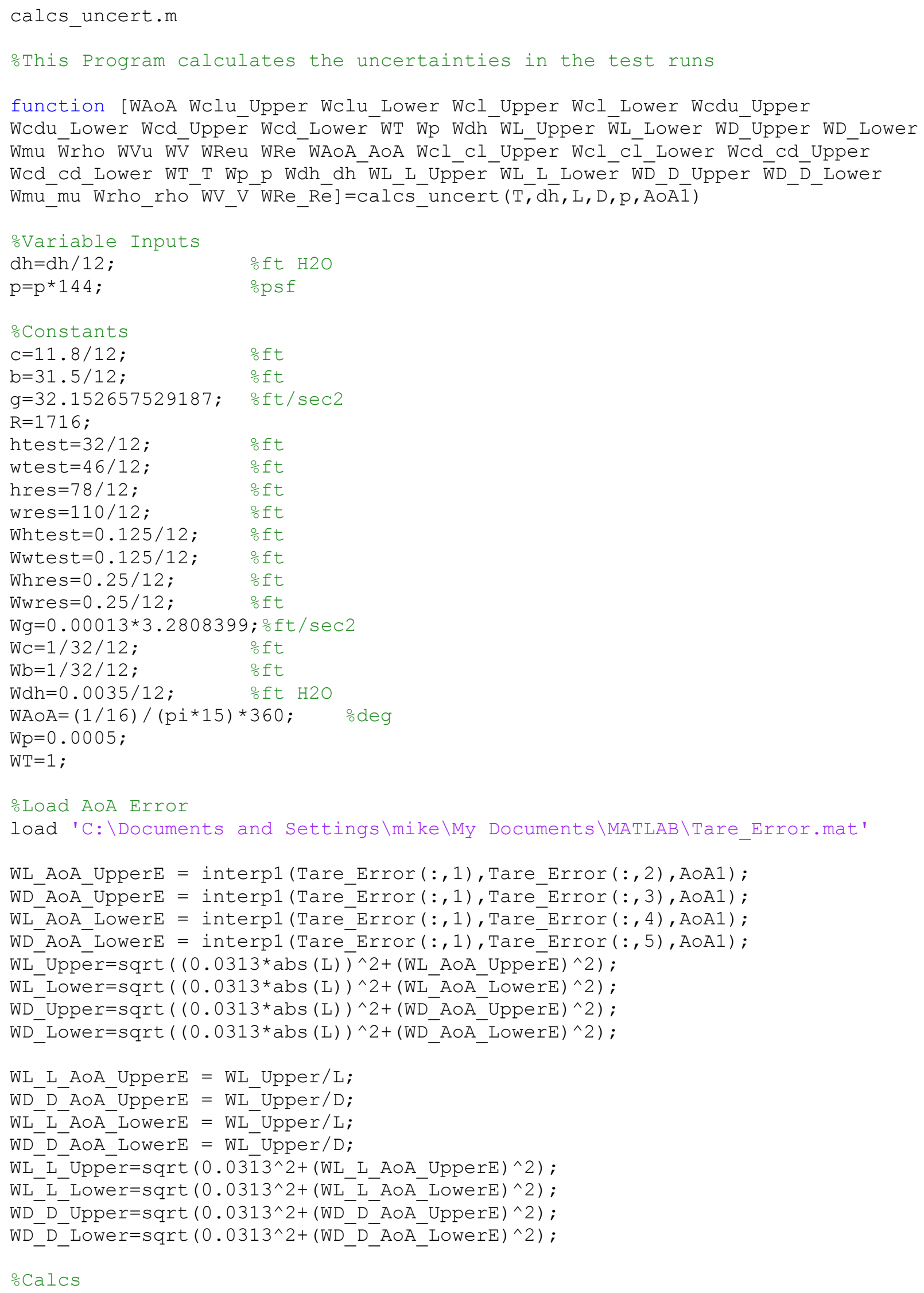




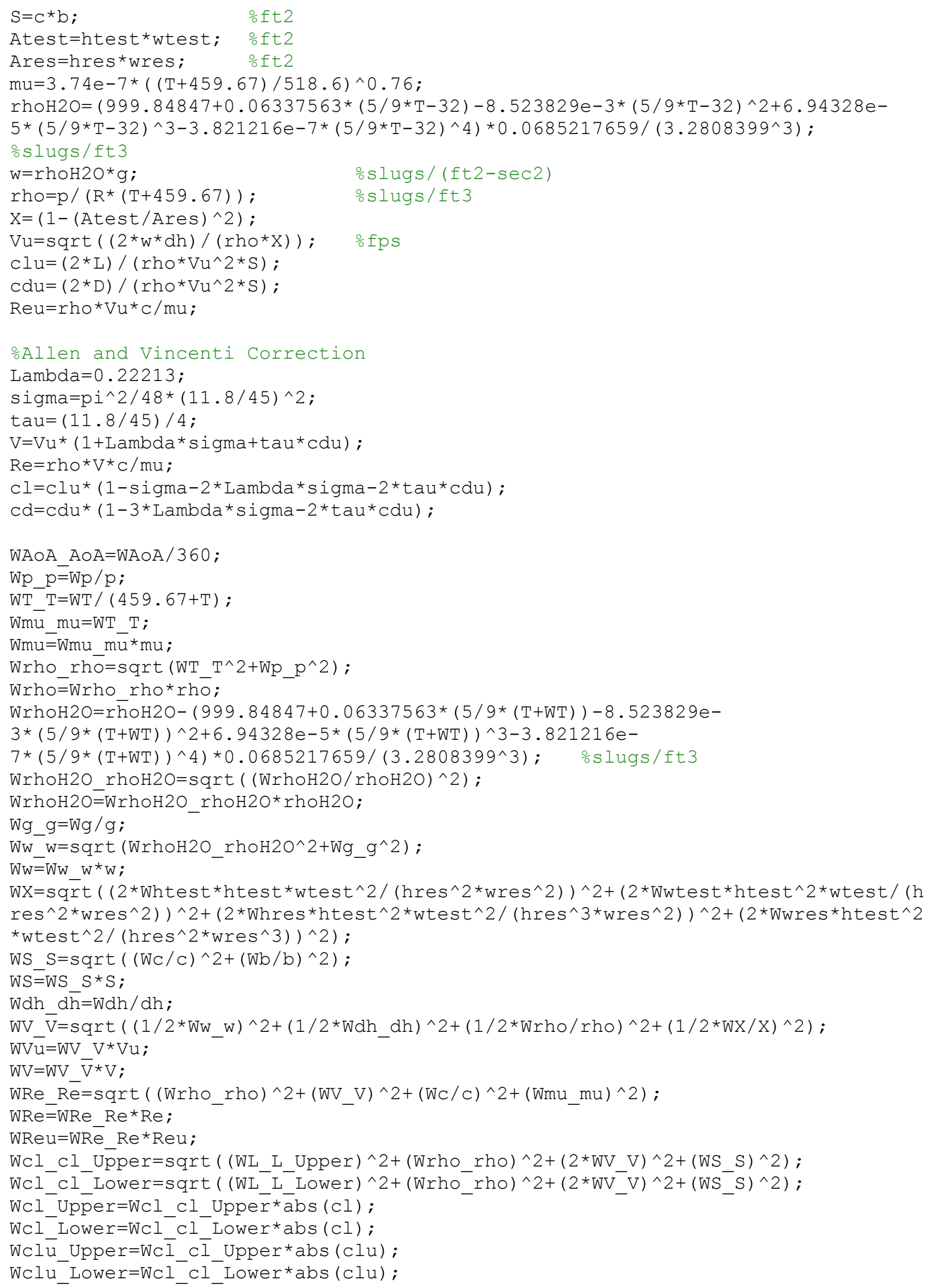


Wcd cd Upper=sqrt $\left((\text { WD D Upper })^{\wedge} 2+(\text { Wrho rho })^{\wedge} 2+(2 * W V \text { V })^{\wedge} 2+(\text { WS } S)^{\wedge} 2\right)$;

$W^{-}{ }^{-} \mathrm{Cd}^{-}$Lower=sqrt $\left(\left(\mathrm{WD}^{-} \mathrm{D}^{-} \text {Lower }\right)^{\wedge} 2+\left(\mathrm{Wrho}^{-} \text {rho }\right)^{\wedge} 2+\left(2 \mathrm{WV}^{-} \mathrm{V}^{\wedge}\right)^{\wedge} 2+\left(\mathrm{WS}^{-} \mathrm{S}^{\wedge}\right)^{\wedge} 2\right)$;

Wcd_Upper $=$ Wcd_cd_Upper ${ }^{*} \bar{a}$ bs $(c d)$;

Wcd Lower=Wcd ${ }^{-}{ }^{-}$Lower*abs (cd);

Wcdu_Upper $=$ Wcd_co

Wcdu_Lower=Wcd_cd_Lower*abs (cdu) ;

importer_neg.m

oThis file is identical to importer.m with the exception that the angle of attack is multiplied by -1 . 